\title{
Brownian motors: noisy transport far from equilibrium ${ }^{1}$
}

\author{
Peter Reimann \\ Universität Augsburg, Institut für Physik, Universitätsstr. 1, 86135 Augsburg, Germany
}

August 2001

${ }^{1}$ Revised version, accepted for publication in Physics Reports 


\section{Contents}

$\begin{array}{lll}1 & \text { Introduction } & 1\end{array}$

1.1 Outline and Scope . . . . . . . . . . . . . . . . . . . . . . 1

1.2 Historical landmark . . . . . . . . . . . . . . . . . . . 2

1.3 Organization of the paper . . . . . . . . . . . . . . . . . . 4

$\begin{array}{lll}2 & \text { Basic concepts and phenomena } & 6\end{array}$

2.1 Smoluchowski-Feynman ratchet . . . . . . . . . . . . . . . . . 6

2.1 .1 Ratchet and paw . . . . . . . . . . . . . . . . . 6

$2.1 .2 \quad$ Simplified stochastic mode . . . . . . . . . . . . . . . 8

2.2 Fokker-Planck equation $\ldots \ldots \ldots \ldots$. . . . . . . . . . . . 10

2.3 Particle current . . . . . . . . . . . . . . . . . . . . . . . . . . . 11

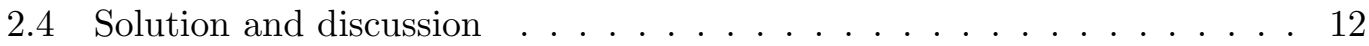

2.5 Tilted Smoluchowski-Feynman ratchet . . . . . . . . . . . . . . . . . . . 15

2.5 .1 Weak noise limit . . . . . . . . . . . . . . . . . 18

2.6 Temperature ratchet and ratchet effect . . . . . . . . . . . . . . . . . 19

2.6 .1 Model . . . . . . . . . . . . . . . . . . . . 19

2.6 .2 Ratchet effect . . . . . . . . . . . . . . . . . . . 20

2.6 .3 Discussion . . . . . . . . . . . . . . . . . . . . 21

2.7 Mechanochemical coupling . . . . . . . . . . . . . . . . . . . 23

2.8 Curie's principle . . . . . . . . . . . . . . . . . . . . . . . . . 24

2.9 Brillouin's paradox . . . . . . . . . . . . . . . . . . . . 25

2.10 Asymptotic analysis . . . . . . . . . . . . . . . . 26

2.11 Current inversions $\ldots \ldots \ldots \ldots \ldots \ldots \ldots$

3 General Framework $\quad 32$

3.1 Working model . . . . . . . . . . . . . . . . . . . . . . 32

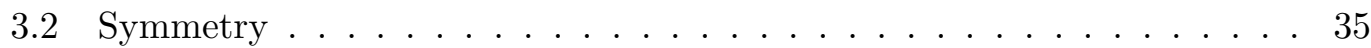

3.2 .1 Definitions . . . . . . . . . . . . . . . . 35

3.2 .2 Conclusions . . . . . . . . . . . . . . . . . . 36

3.3 Main ratchet types . . . . . . . . . . . . . . . . . . . . 37

3.4 Physical basis . . . . . . . . . . . . . . . . . . . . . . . . . . . . . . 39

3.4.1 Thermal environment . . . . . . . . . . . . . . . . . . 40

3.4 .2 Nonequilibrium perturbations . . . . . . . . . . . . . . . . . . 44

3.5 Supersymmetry . . . . . . . . . . . . . . . . . . 46

3.5 .1 Definitions . . . . . . . . . . . . . . . . . 46

3.5 .2 Main conclusion . . . . . . . . . . . . . . . . . . . . . . . . . . . . . . . . . . . 47

3.5 .3 Examples . . . . . . . . . . . . . . . . . . . . . . . . . . . . . 48

3.5.4 Discussion . . . . . . . . . . . . . . . . . . 51 
3.5 .5 Generalizations . . . . . . . . . . . . . . . . . . . . 52

3.6 Tailoring current inversions $\ldots \ldots \ldots \ldots \ldots$

3.7 Linear response and high temperature limit . . . . . . . . . . . . . . . 54

3.8 Activated barrier crossing limit . . . . . . . . . . . . . . . . . 55

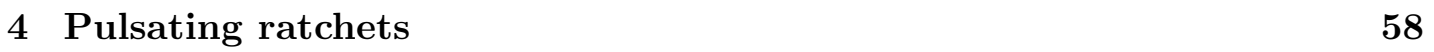

4.1 Fast and slow pulsating limits . . . . . . . . . . . . . . . 58

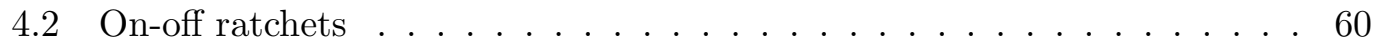

4.2 .1 Experimental realizations . . . . . . . . . . . . . . 61

4.3 Fluctuating potential ratchet . . . . . . . . . . . . . . . . . . . . 62

4.3 .1 Dichotomous potential fluctuations . . . . . . . . . . . . . 63

$4.3 .2 \quad$ Gaussian potential fluctuations . . . . . . . . . . . . . . . . 65

4.4 Traveling potential ratchets . . . . . . . . . . . . . . . . . . . . . . . . . . . . 68

4.4 .1 Genuine traveling potentials . . . . . . . . . . . . . 68

4.4 .2 Improper traveling potentials . . . . . . . . . . . . . . 71

4.5 Hybrids and further generalizations . . . . . . . . . . . . . . . . 74

4.5 .1 Superpositions of traveling potentials . . . . . . . . . . . . 74

4.5.2 Generalized pulasting ratchets and experimental realizations . . 76

4.6 Biological applications: molecular pumps and motors . . . . . . . . . . . 78

4.6 .1 Externally driven molecular pumps . . . . . . . . . . . . . . 79

$\begin{array}{llr}5 & \text { Tilting ratchets } & \mathbf{8 1}\end{array}$

5.0 .2 Model . . . . . . . . . . . . . . . . . . . . 81

5.0 .3 Adiabatic approximation . . . . . . . . . . . . . 81

5.0 .4 Fast tilting . . . . . . . . . . . . . . . . 83

5.0 .5 Comparison with pulsating ratchets . . . . . . . . . . . . . 84

5.1 Fluctuating force ratchets $\ldots \ldots \ldots \ldots$. . . . . . . . . . . 85

5.1 .1 Fast fluctuating forces . . . . . . . . . . . . . . . . . . 86

5.1 .2 Specific types of fluctuating forces . . . . . . . . . . . . . . . . 89

5.2 Photovoltaic effects . . . . . . . . . . . . . . . . . . . . 91

5.3 Rocking ratchets . . . . . . . . . . . . . . . . . . . . . . . . 93

5.3 .1 Fast rocking limit . . . . . . . . . . . . . . . . . . . . 93

5.3 .2 General qualitative features . . . . . . . . . . . . . . . . . 95

5.3 .3 Applications . . . . . . . . . . . . . . . . 96

5.4 Influence of inertia and Hamiltonian ratchets . . . . . . . . . . . . 97

5.5 Two-dimensional systems and entropic ratchets . . . . . . . . . . . . . 99

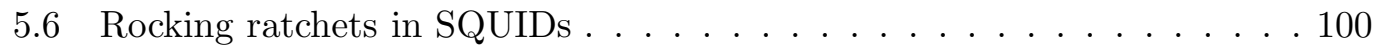

5.7 Giant enhancement of diffusion . . . . . . . . . . . . . . . . . . . . 103

5.8 Asymmetrically tilting ratchets . . . . . . . . . . . . . . . . . . . 105

$5.8 .1 \quad$ Periodic driving . . . . . . . . . . . . . . . . . . 105

$5.8 .2 \quad$ Stochastic, chaotic, and quasiperiodic driving . . . . . . . . . 107

$\begin{array}{llr}6 & \text { Sundry Extensions } & 109\end{array}$

6.1 Seebeck ratchets . . . . . . . . . . . . . . . . . . . . . . 109

6.2 Feynman ratchets . . . . . . . . . . . . . . . . . . . . . . . . . 111

6.3 Temperature ratchets . . . . . . . . . . . . . . . . . . . 113

6.4 Inhomogeneous, pulsating, and memory friction . . . . . . . . . . . 115

6.4 .1 A no-go theorem . . . . . . . . . . . . . . . 115 
6.4 .2 Inhomogeneous and pulsating friction $\ldots \ldots . \ldots . \ldots 116$

6.4 .3 Memory friction and correlated thermal noise . . . . . . . . . . . 117

6.5 Ratchet models with an internal degree of freedom . . . . . . . . . . . . 118

6.6 Drift ratchet $\ldots \ldots \ldots \ldots$

6.7 Spatially discrete models and Parrondo's game . . . . . . . . . . . . . . 122

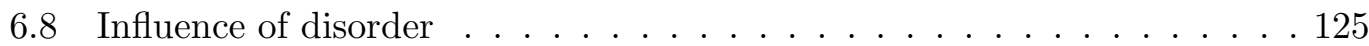

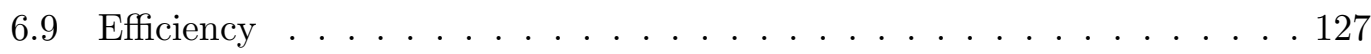

\begin{tabular}{lll}
\hline & Molecular motors & $\mathbf{1 3 0}$
\end{tabular}

7.1 Biological setup . . . . . . . . . . . . . . . . . . . 130

$7.2 \quad$ Basic modeling-steps . . . . . . . . . . . . . . . . . . . . . . . . . . 132

7.2 .1 Biochemical framework . . . . . . . . . . . . . . 132

7.2 .2 Mechanical and chemical state variables . . . . . . . . . . . . . 134

$7.2 .3 \quad$ Discrete chemical states . . . . . . . . . . . . . . . . 135

7.3 Simplified stochastic model . . . . . . . . . . . . . . . . . 136

$7.3 .1 \quad$ Stochastic ratchet dynamics . . . . . . . . . . . . . . . . . 137

7.3 .2 Nonequilibrium chemical reaction . . . . . . . . . . . . . . . . . . 140

7.4 Collective one-head models . . . . . . . . . . . . . . . . . . . 142

$7.4 .1 \quad$ A.F. Huxley's mode . . . . . . . . . . . . . . . . . . . . . . . . . 142

7.4 .2 Free choice of chemical reaction rates . . . . . . . . . . . . . 144

$7.4 .3 \quad$ Generalizations . . . . . . . . . . . . . . . . . . 145

7.4 .4 Jülicher-Prost model . . . . . . . . . . . . . . . . . . . . . . . . . 147

7.5 Coordinated two-head model . . . . . . . . . . . . . . . . 150

7.6 Further models for a single motor enzyme . . . . . . . . . . . . . . . . 152

7.7 Summary and discussion . . . . . . . . . . . . . . . . . . . 154

8 Quantum ratchets 158

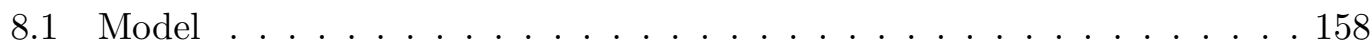

8.2 Adiabatically tilting quantum ratchet . . . . . . . . . . . . . . . . . 161

8.2 .1 Tunneling induced current inversion . . . . . . . . . . . . . . 166

8.3 Beyond the adiabatic limit . . . . . . . . . . . . . . . . . . . 168

8.4 Experimental quantum ratchet systems . . . . . . . . . . . . . . 170

$\begin{array}{llr}9 & \text { Collective effects } & \mathbf{1 7 5}\end{array}$

9.1 Coupled ratchets . . . . . . . . . . . . . . . . . . . 176

9.2 Genuine collective effects . . . . . . . . . . . . . . . . . 178

9.2 .1 Model . . . . . . . . . . . . . . . . . . . . . . . . 179

9.2 .2 Spontaneous symmetry breaking . . . . . . . . . . . . . . . . 179

$9.2 .3 \quad$ Spontaneous ratchet effect . . . . . . . . . . . . . . . . . . 182

$9.2 .4 \quad$ Negative mobility and anomalous hysteresis . . . . . . . . . . . . 184

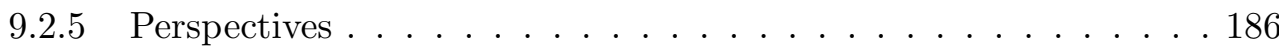

\begin{tabular}{ll}
\hline 10 Conclusions & 189
\end{tabular}

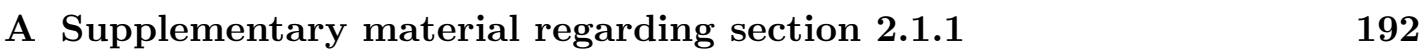

A.1 Gaussian white noise . . . . . . . . . . . . . . . . . . . . . 192

A.2 $\quad$ Fluctuation-dissipation relation . . . . . . . . . . . . . . . . . . . . . 193

A.3 Einstein relation . . . . . . . . . . . . . . . . . . . . . . . . 194

A.4 Dimensionless units and overdamped dynamics . . . . . . . . . . . . . . 194 
\begin{tabular}{|l|l|l|}
\hline B Alternative derivation of the Fokker-Planck equation & 197
\end{tabular}

\begin{tabular}{ll}
\hline C Perturbation analysis & 198
\end{tabular} 


\begin{abstract}
Transport phenomena in spatially periodic systems far from thermal equilibrium are considered. The main emphasize is put on directed transport in so-called Brownian motors (ratchets), i.e. a dissipative dynamics in the presence of thermal noise and some prototypical perturbation that drives the system out of equilibrium without introducing a priori an obvious bias into one or the other direction of motion. Symmetry conditions for the appearance (or not) of directed current, its inversion upon variation of certain parameters, and quantitative theoretical predictions for specific models are reviewed as well as a wide variety of experimental realizations and biological applications, especially the modeling of molecular motors. Extensions include quantum mechanical and collective effects, Hamiltonian ratchets, the influence of spatial disorder, and diffusive transport.
\end{abstract}

PACS: 5.40.-a, 5.60.-k, 87.16.Nn 


\section{Chapter 1}

\section{Introduction}

\subsection{Outline and Scope}

The subject of the present review are transport phenomena in spatially periodic systems out of thermal equilibrium. While the main emphasis is put on directed transport, also some aspects of diffusive transport will be addressed. We furthermore focus mostly on small-scale systems for which thermal noise plays a non-negligible or even dominating role. Physically, the thermal noise has its origin in the thermal environment of the actual system of interest. As an unavoidable consequence, the system dynamics is then always subjected to dissipative effects as well.

Apart from transients, directed transport in a spatially periodic system in contact with a single dissipation- and noise-generating thermal heat bath is ruled out by the second law of thermodynamics. The system has therefore to be driven away from thermal equilibrium by an additional deterministic or stochastic perturbation. Out of the infinitely many options, we will mainly focus on either a periodic driving or a restricted selection of stochastic processes of prototypal simplicity. In the most interesting case, these perturbations are furthermore unbiased, i.e. the time-, space-, and ensemble-averaged forces which they entail are required to vanish. Physically, they may be either externally imposed (e.g. by the experimentalist) or of system-intrinsic origin, e.g. due to a second thermal heat reservoir at a different temperature or a non-thermal bath.

Besides the breaking of thermal equilibrium, a further indispensable requirement for directed transport in spatially periodic systems is clearly the breaking of the spatial inversion symmetry. There are essentially three different ways to do this, and we will speak of a Brownian motor, or equivalently, a ratchet system whenever a single one or a combination of them is realized: First, the spatial inversion symmetry of the periodic system itself may be broken intrinsically, that is, already in the absence of the above mentioned non-equilibrium perturbations. This is the most common situation and typically involves some kind of periodic and asymmetric, so-called ratchet-potential. A second option is that the non-equilibrium perturbations, notwithstanding the requirement that they must be unbiased, bring about a spatial asymmetry of the dynamics. A third possibility arises as a collective effect in coupled, perfectly symmetric nonequilibrium systems, namely in the form of spontaneous symmetry breaking.

As it turns out, these two conditions (breaking of thermal equilibrium and of spatial inversion symmetry) are generically sufficient for the occurrence of the so-called ratchet effect, i.e. the emergence of directed transport in a spatially periodic system. 
Elucidating this basic phenomenon in all its facets is the central theme of our present review.

We will mainly focus on two basic classes of ratchet systems, which may be roughly characterized as follows (for a more detailed discussion see section 3.3): The first class, called pulsating ratchets, are those for which the above mentioned periodic or stochastic non-equilibrium perturbation gives rise to a time-dependent variation of the potential shape without affecting its spatial periodicity. The second class, called tilting ratchets, are those for which these non-equilibrium perturbations act as an additive driving force, which is unbiased on the average. In full generality, also combinations of pulsating and tilting ratchet schemes are possible, but they exhibit hardly any fundamentally new basic features (see section 3.4.2). Even within those two classes, the possibilities of breaking thermal equilibrium and symmetry in a ratchet system are still numerous and in many cases, predicting the actual direction of the transport is already far from obvious, not to speak of its quantitative value. In particular, while the occurrence of a ratchet effect is the rule, exceptions with zero current are still possible. For instance, such a non-generic situation may be created by fine-tuning of some parameter. Usually, the direction of transport then exhibits a change of sign upon variation of this parameter, called current inversions. Another type of exception can be traced back to symmetry reasons with the characteristic signature of zero current without fine tuning of parameters. The understanding and control of such exceptional cases is clearly another issue of considerable theoretical and practical interest that we will discuss in detail (especially in sections 3.5 and 3.6).

\subsection{Historical landmarks}

Progress in the field of Brownian motors has evolved through contributions from rather different directions and re-discoveries of the same basic principles in different contexts have been made repeatedly. Moreover, the organization of the much more detailed subsequent chapters will not always admit it to keep the proper historical order. For these reasons, a brief historical tour d'horizon seems worthwhile at this place. At the same time, this gives a first flavor of the wide variety of applications of Brownian motor concepts.

Though certain aspects of the ratchet effect are contained implicitly already in the works of Archimedes, Seebeck, Maxwell, Curie, and others, Smoluchowski's Gedankenexperiment from 1912 [1] regarding the prima facie quite astonishing absence of directed transport in spatially asymmetric systems in contact with a single heat bath, may be considered as the first seizable major contribution (discussed in detail in section 2.1). The next important step forward represents Feynman's famous recapitulation and extension [2] to the case of two thermal heat baths at different temperatures (see section 6.2).

Brillouins paradox [3] from 1950 (see section 2.9) may be viewed as a variation of Smoluchowski's counterintuitive observation. In turn, Feynman's prediction that in the presence of a second heat bath a ratchet effect will manifest itself, has its Brillouintype correspondence in the Seebeck effect (see section 6.1), discovered by Seebeck in 1822 of course without any idea about the underlying microscopic ratchet effect.

Another root of Brownian motor theory leads us into the realm of intracellular transport research, specifically the biochemistry of molecular motors and molecular pumps. In the case of molecular motors, the concepts which we have in mind here have 
been unraveled in several steps, starting with A. Huxley's ground-breaking 1957 work on muscle contraction [4], and continued in the late 80-s by Braxton and Yount [5, 6] and in the 90-s by Vale and Oosawa [7], Leibler and Huse [8, 9], Cordova, Ermentrout, and Oster [10], Magnasco [11, 12], Prost, Ajdari, and collaborators [13, 14], Astumian and Bier [15, 16], Peskin, Ermentrout, and Oster [17, 18] and many others, see chapter 7 . In the case of molecular pumps, the breakthrough came with the theoretical interpretation of previously known experimental findings [19,20] as a ratchet effect in 1986 by Tsong, Astumian and coworkers [21, 22], see section 4.6. While the general importance of asymmetry induced rectification, thermal fluctuations, and the coupling of non-equilibrium enzymatic reactions to mechanical currents according to Curie's principle for numerous cellular transport processes is long known [23, 24], the above works introduced for the first time a quantitative microscopic modeling beyond the linear response regime close to thermal equilibrium.

On the physical side, a ratchet effect in the form of voltage rectification by a dcSQUID in the presence of a magnetic field and an unbiased ac-current (i.e. a tilting ratchet scheme) has been experimentally observed and theoretically interpreted as early as in 1967 by De Waele, Kraan, de Bruyn Ouboter, and Taconis [25, 26]. Further, directed transport induced by unbiased, time-periodic driving forces in spatially periodic structures with broken symmetry has been the subject of several hundred experimental and theoretical papers since the mid 70-s. In this context of the so-called photovoltaic and photorefractive effects in non-centrosymmetric materials, a ground breaking experimental contribution represents the 1974 paper by Glass, von der Linde, and Negran [27]. The general theoretical framework was elaborated a few years later by Belinicher, Sturman and coworkers, as reviewed - together with the above mentioned numerous experiments - in their capital works [28,29]. They identified as the two main ingredients for the occurrence of the ratchet effect in periodic systems the breaking of thermal equilibrium (detailed balance symmetry) and of the spatial symmetry, and they pointed out the much more general validity of such a tilting ratchet scheme beyond the specific experimental systems at hand, see section 5.2 .

The possibility of producing a dc-output by two superimposed sinusoidal ac-inputs at frequencies $\omega$ and $2 \omega$ in a spatially periodic, symmetric system, exemplifying a so-called asymmetrically tilting ratchet mechanism, has been observed experimentally 1978 by Seeger and Maurer [30] and analyzed theoretically 1979 by Wonneberger [31], see section 5.8.1. The occurrence of a ratchet effect has been theoretically predicted 1987 by Bug and Berne [32] for the simplest variant of a pulsating ratchet scheme, termed on-off ratchet (see section 4.2). A ratchet model with a symmetric periodic potential and a state-dependent temperature (multiplicative noise) with the same periodicity but out of phase, i.e. a simplified microscopic model for the Seebeck effect (see section 6.1), has been analyzed 1987 by Büttiker [33].

The independent re-inventions of the on-off ratchet scheme 1992 by Ajdari and Prost [34] and of the tilting ratchet scheme 1993 by Magnasco [1] together with the seminal 1994 works (ordered by date of receipt) 13, 15, 35 [37, [12, 38, 40], [17], 41, 42] provided the inspiration for a whole new wave of great theoretical and experimental activity and progress within the statistical physics community as detailed in the subsequent chapters and reviewed e.g. in [14,43 61]. While initially the modeling of molecular motors has served as one of the main motivations, the scope of Brownian motor studies has subsequently been extended to an ever increasing number of physical and technological applications, along with the re-discovery of the numerous pertinent 
works from before 1992. As a result, a much broader and unified conceptual basis has been achieved, new theoretical tools have been developed which lead to the discovery of many interesting and quite astonishing effects, and a large variety of exciting new experimental realizations have become available.

Within the realm of noise-induced or -assisted non-equilibrium phenomena, an entire family of well-established major fields are known under the labels of stochastic resonance [62], noise induced transitions [63] and phase transitions [64, 65, reaction rate theory [66 68], and driven diffusive systems [69, 70], to name but a few examples. One objective of our present review is to show that the important recent contributions of many workers to the theory and application of Brownian motors has given rise to another full-fledged member of this family.

\subsection{Organization of the paper}

This review addresses two readerships: It may serve as an introduction to the field without requiring any specialized preknowledge. On the other hand, it offers to the active researcher a unifying view and guideline through the very rapidly growing literature. For this reason, not everything will be of equal interest for everybody. The following outline together with the table of contents may help to make one's selection.

Essentially, the subsequent 8 chapters can be divided into three units of rather different characters: The first unit (chapter 2) is predominantly of introductory and pedagogical nature, illustrating the basic phenomena, concepts, and applications by way of examples. Technically, the discussion is conducted on a rather elementary level and the calculations are to a far extent self-contained. "Standard" lines of reasoning and the derivation of basic working tools are discussed rather detailed in mathematically heuristic but physically suggestive terms. While these parts of chapter 2 are not meant to replace a systematic introduction to the field of stochastic processes, they may hopefully serve as a minimal basis for the technically less detailed subsequent chapters.

Chapter 3 is devoted to general and systematic considerations which are relevant for the entire subsequent parts of the paper. The main classes of ratchet models and their physical origin are discussed with particular emphasize on symmetries, current inversions, and asymptotic regimes. Chapters 4 -6 represent the main body of the present work and are to a large extent of review character. It was only during the completion of these chapters that the amount of pertinent literature in this context became clear. As a consequence, specific new aspects of the considered ratchet systems and of the obtained results could only be included for a selection of particularly significant such studies. Even then, the technical procedures and the detailed quantitative findings had to be mostly omitted. Besides the conceptual theoretical considerations and the systematic discussion of various specific model classes, a substantial part of chapters 46 has also been reserved for the manifold experimental applications of those ideas.

Chapters $7-9$ represent the third main unit of our work, elaborating in somewhat more detail three major instances of applications and extensions. Of methodic rather than review character are the first 3 sections of chapter 7 , illustrating a typical stochastic modeling procedure for the particularly important example of intracellular transport processes by molecular motors. The remainder of chapter 7 presents a survey of the field with particular emphasize on cooperative molecular motors and the character of the mechanochemical coupling. Chapter 8 is devoted to the discussion of theoreti- 
cally predicted new characteristic quantum mechanical signatures of Brownian motors and their experimental verification on the basis of a quantum dot array with broken spatial symmetry. Finally, chapter 9 deals with collective effects of interacting ratchet systems. On the one hand, we review modifications of the directed transport properties of single ratchets caused by their interaction (section 9.1). On the other hand (section 9.2) we exemplify genuine collective transport phenomena by a somewhat more detailed discussion of one specific model of paradigmatic simplicity - meant as a kind of "normal form" description which still captures the essence of more realistic models but omits all unnecessary details, in close analogy to the philosophy usually adopted in the theory of equilibrium phase transitions.

Concluding remarks and future perspectives are presented in chapter 10. Some technical details from the introductory chapter 2 are contained in the appendices.

Previously unpublished research represent the considerations about supersymmetry in section 3.5, the method of tailoring current inversions in section 3.6, the general treatment of the linear response regime in section 3.7, the approximative molecular motor model with two highly cooperative "heads" in section 7.5, as well as a number of additional minor new results which are indicated as such throughout the text, e.g., various exact mappings between different classes of ratchet systems. New, mainly by the way of presentation but to some degree also by their content, are parts of sections 2.1 2.4 and 6.1-6.4, the systematic ratchet classification scheme and its physical basis in sections 3.3 and 3.4, the unified fast fluctuating force asymptotics in section 5.1.1, as well as the coherent historical review in the preceding section 1.2 .

A kind of red thread through the entire review consists in the asymptotic analysis of the so-called fast-driving limit. By collecting and rewriting the various results spread out in the literature and completing the missing pieces, a unified picture of this asymptotic regime emerges for the first time. The structural similarity of these analytical results in view of the rather different underlying models is remarkable. For instance, within our standard working model - the overdamped Brownian motion in a periodic non-equilibrium system involving some ratchet-potential $V(x)$ of period $L$ - the direction of the average particle current is governed under very general circumstances by a factor of the form $\int_{0}^{L} V^{\prime}(x)\left[d^{n} V(x) / d x^{n}\right]^{2} d x$ with a model dependent $n$-value between 1 and 3. Especially, already within this asymptotic regime, the intriguingly complicated dependence of the directed transport, e.g. on the detailed potential-shape $V(x)$, becomes apparent - a typical feature of systems far from thermal equilibrium.

Basically, the review is organized in three levels (chapters, sections, subsections). While from the logical viewpoint, additional levels would have been desirable, the present rather "flat" structure simplifies a quick orientation on the basis of the table of contents. Throughout the main text, cross-referencing to related subsections is used rather extensively. It may be ignored in case of a systematic reading, but is hopefully of use otherwise. 


\section{Chapter 2}

\section{Basic concepts and phenomena}

This chapter serves as a motivation and first exposition of the main themes of our review, such as the absence of directed transport in ratchet systems at thermal equilibrium, its generic occurrence away from equilibrium, and the possibility of current inversions upon variation of some parameter. These fundamental phenomena are exemplified in their simplest form in section 2.1, sections 2.6-2.9, and section 2.11, respectively, and will then be elaborated in more generality and depth in the subsequent chapters. At the same time, this chapter also introduces the basic stochastic modeling concepts as well as the mathematical methods and "standard arguments" in this context. These issues are mainly contained in sections 2.2 2.5 and 2.10 , complemented by further details in the respective appendices. Readers which are already familiar with these basic physical phenomena and mathematical concepts may immediately proceed to chapter 3 .

\subsection{Smoluchowski-Feynman ratchet}

Is it possible, and how is it possible to gain useful work out of unbiased random fluctuations? In the case of macroscopic fluctuations, the task can indeed be accomplished by various well-known types of mechanical and electrical rectifiers. Obvious daily-life examples are the wind-mill or the self-winding wristwatch. More subtle is the case of microscopic fluctuations, as demonstrated by the following Gedankenexperiment about converting Brownian motion into useful work. The basic idea can be traced back to a conference talk by Smoluchowski in Münster 1912 (published as proceedings-article in Ref. [1]) and was later popularized and extended in Feynman's Lectures on Physics [2].

\subsubsection{Ratchet and pawl}

The main ingredient of Smoluchowski and Feynman's Gedankenexperiment is an axle with at one end paddles and at the other end a so-called ratchet, reminiscent of a circular saw with asymmetric saw-teeth (see figure 2.1). The whole device is surrounded by a gas at thermal equilibrium. So, if it could freely turn around, it would perform a rotatory Brownian motion due to random impacts of gas molecules on the paddles. The idea is now to rectify this unbiased random motion with the help of a pawl (see figure 2.1). It is indeed quite suggestive that the pawl will admit the saw-teeth to proceed without much effort into one direction (henceforth called "forward") but practically exclude a rotation in the opposite ("backward") direction. In other words, it 
seems quite convincing that the whole gadget will perform on the average a systematic rotation in one direction, and this in fact even if a small load in the opposite direction is applied.

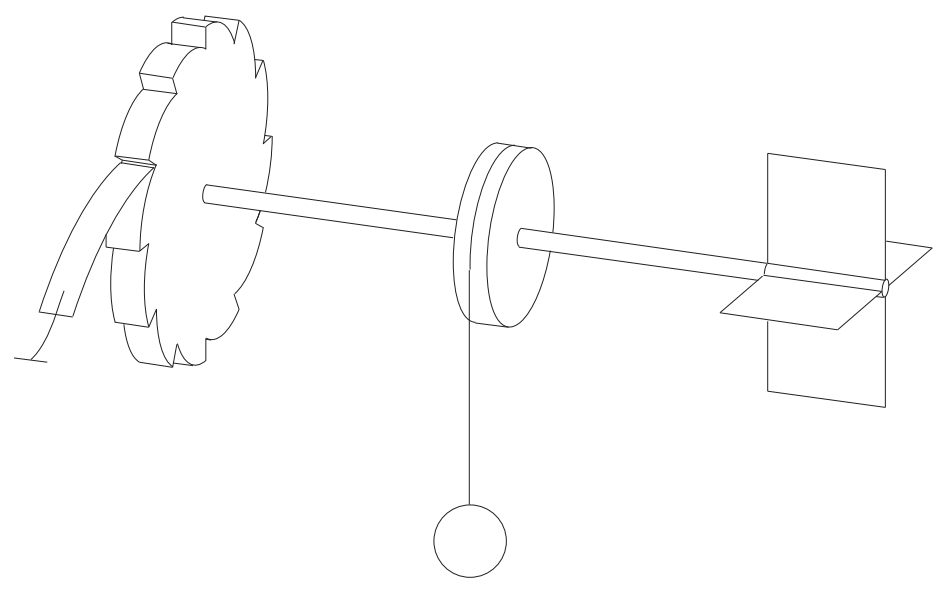

Figure 2.1: Ratchet and pawl. The ratchet is connected by an axle with the paddles and with a spool, which may lift a load. In the absence of the pawl (leftmost object) and the load, the random collisions of the surrounding gas molecules (not shown) with the paddles cause an unbiased rotatory Brownian motion. The pawl is supposed to rectify this motion so as to lift the load.

Astonishingly enough, this naive expectation is wrong: In spite of the built in asymmetry, no preferential direction of motion is possible. Otherwise, such a gadget would represent a perpetuum mobile of the second kind, in contradiction to the second law of thermodynamics. The culprit must be our assumption about the working of the pawl, which is indeed closely resembling Maxwell's demon円. Since the impacts of the gas molecules take place on a microscopic scale, the pawl needs to be extremely small and soft in order to admit a rotation even in the forward direction. As Smoluchowski points out, the pawl itself is therefore also subjected to non-negligible random thermal fluctuations. So, every once in a while the pawl lifts itself up and the saw-teeth can freely travel underneath. Such an event clearly favors on the average a rotation in the "backward" direction in figure 2.1. At overall thermal equilibrium (the gas surrounding the paddles and the pawl being at the same temperature) the detailed quantitative analysis [2] indeed results in the subtle probabilistic balance which just rules out the functioning of such a perpetuum mobile.

A physical system as described above will be called after Smoluchowski and Feynman. We will later go one step further and consider the case that the gas surrounding the paddles and the pawl are not at the same temperature (see section 6.2). Such an

\footnotetext{
${ }^{1}$ Both Smoluchowski and Feynman have pointed out the similarity between the working principle of the pawl and that of a valve. A valve, acting between two boxes of gas, is in turn one of the simplest realizations of a Maxwell demon [71. For more details on Maxwell's demon, especially the history of this apparent paradox and its resolution, we refer to the commented collection of reprints in [72].
} 
extension of the original Gedankenexperiment appears in Feynman's lectures, but has not been discussed by Smoluchowski, and will therefore be named after Feynman only.

Smoluchowski and Feynman's ratchet and pawl has been experimentally realized on a molecular scale by Kelly, Tellitu, and Sestelo [73 76$]$. Their synthesis of triptycene[4] helicene incorporates into a single molecule all essential components: The triptycene "paddlewheel" functions simultaneously as circular ratchet and as paddles, the helicene serves as pawl and provides the necessary asymmetry of the system. Both components are connected by a single chemical bond, giving rise to one degree of internal rotational freedom. By means of sophisticated NMR (nuclear magnetic resonance) techniques, the predicted absence of a preferential direction of rotation at thermal equilibrium has been confirmed experimentally. The behavior of similar experimental systems beyond the realm of thermal equilibrium will be discussed at the end of section 4.5 .2 .

\subsubsection{Simplified stochastic model}

In the sense that we are dealing merely with a specific instance of the second law of thermodynamics, the situation with respect to Smoluchowski-Feynman's ratchet and pawl is satisfactorily clarified. On the other hand, the obvious intention of Smoluchowski and Feynman is to draw our attention to the amazing content and implications of this very law, calling for a more detailed explanation of what is going on. A satisfactory modeling and analysis of the relatively complicated ratchet and pawl gadget as it stands is possible but rather involved, see section 6.2. Therefore, we focus on a considerably simplified model which, however, still retains the basic qualitative features: We consider a Brownian particle in one dimension with coordinate $x(t)$ and mass $m$, which is governed by Newton's equation of motion?:

$$
m \ddot{x}(t)+V^{\prime}(x(t))=-\eta \dot{x}(t)+\xi(t) .
$$

Here $V(x)$ is a periodic potential with period $L$,

$$
V(x+L)=V(x)
$$

and broken spatial symmetry thus playing the role of the ratchet in figure 2.1. A typical example is

$$
V(x)=V_{0}[\sin (2 \pi x / L)+0.25 \sin (4 \pi x / L)],
$$

see figure 2.2 .

The left hand side in (2.1) represents the deterministic, conservative part of the particle dynamics, while the right hand side accounts for the effects of the thermal environment. These are energy dissipation, modeled in (2.1) as viscous friction with friction coefficient $\eta$, and randomly fluctuating forces in the form of the thermal noise $\xi(t)$. These two effects are not independent of each other since they have both the same origin, namely the interaction of the particle $x(t)$ with a huge number of microscopic degrees of freedom of the environment. As discussed in detail in sections A.1 and A.2 of Appendix A, our assumption that the environment is an equilibrium heat bath with temperature $T$ and that its effect on the system can be modeled by means of

\footnotetext{
${ }^{2}$ Dot and prime indicate differentiations with respect to time and space, respectively.

${ }^{3}$ Broken spatial symmetry means that there is no $\Delta x$ such that $V(-x)=V(x+\Delta x)$ for all $x$.
} 
the phenomenological ansatz appearing on the right hand side of (2.1) completely fixes 66, 77 97 all statistical properties of the fluctuations $\xi(t)$ without referring to any microscopic details of the environment (see also sections 2.9, 3.4.1, 8.1). Namely, $\xi(t)$ is a Gaussian white noise of zero mean,

$$
\langle\xi(t)\rangle=0
$$

satisfying the fluctuation-dissipation relation 79 81]

$$
\langle\xi(t) \xi(s)\rangle=2 \eta k_{B} T \delta(t-s),
$$

where $k_{B}$ is Boltzmann's constant, $2 \eta k_{B} T$ is the noise intesity or noise strength, and $\delta(t)$ is Dirac's delta-function. Note that the only particle property which enters the characteristics of the noise is the friction coefficient $\eta$, which may thus be viewed as the coupling strength to the environment.

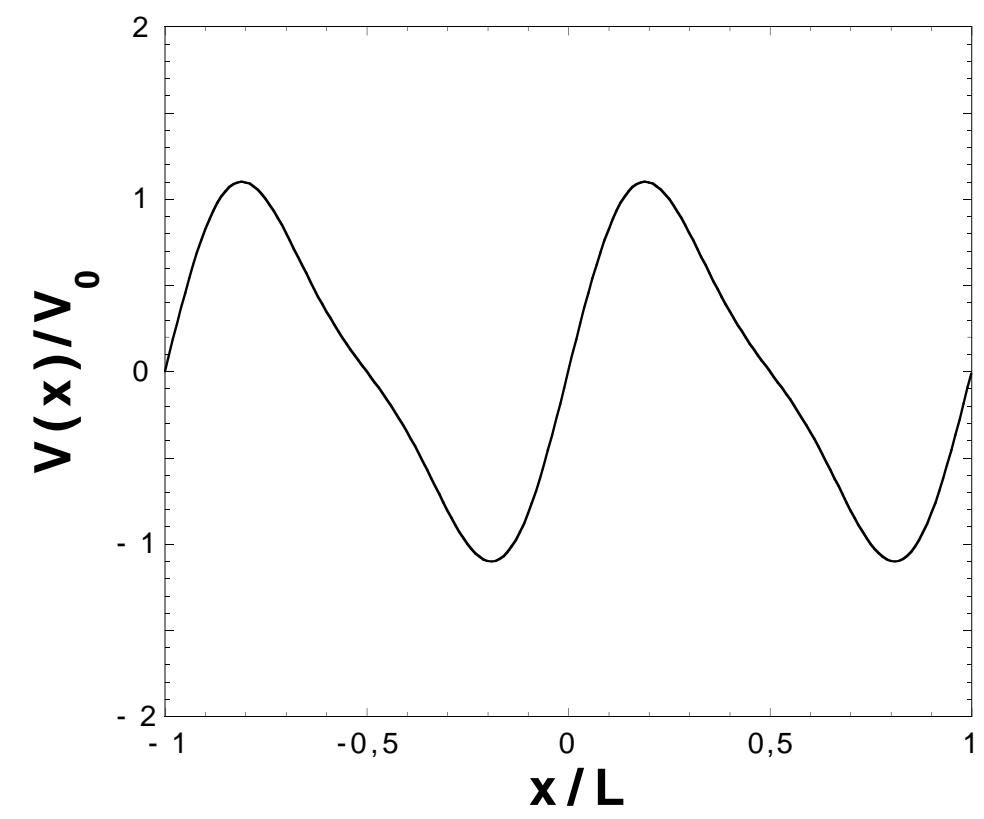

Figure 2.2: Typical example of a ratchet-potential $V(x)$, periodic in space with period $L$ and with broken spatial symmetry. Plotted is the example from (2.3) in dimensionless units.

For the typically very small systems one has in mind, and for which thermal fluctuations play any notable role at all, the dynamics (2.1) is overdamped, that is, the inertia term $m \ddot{x}(t)$ is negligible (see also the more detailed discussion of this point in section A.4 of Appendix A). We thus arrive at our "minimal" Smoluchowski-Feynman ratchet model

$$
\eta \dot{x}(t)=-V^{\prime}(x(t))+\xi(t) .
$$

According to (2.5), the Gaussian white noise $\xi(t)$ is uncorrelated in time, i.e., it is given by independently sampled Gaussian random numbers at any time $t$. This feature 
and the concomitant infinitely large second moment $\left\langle\xi^{2}(t)\right\rangle$ are mathematical idealizations. In physical reality, the correlation time is meant to be finite, but negligibly small in comparison with all other relevant time scales of the system. In this spirit, we may introduce a small time step $\Delta t$ and consider a time-discretized version of the stochastic dynamics (2.6) of the form

$$
x\left(t_{n+1}\right)=x\left(t_{n}\right)-\Delta t\left[V^{\prime}\left(x\left(t_{n}\right)\right)+\xi_{n}\right] / \eta,
$$

where $t_{n}:=n \Delta t$ and where the $\xi_{n}$ are independently sampled, unbiased Gaussian random numbers with second moment

$$
\left\langle\xi_{n}^{2}\right\rangle=2 \eta k_{B} T / \Delta t
$$

The continuous dynamics (2.6) with uncorrelated noise is then to be understood 98 100 as the mathematical limit of (2.7) for $\Delta t \rightarrow 0$. Moreover, this discretized dynamics (2.7) is a suitable starting point for a numerical simulation of the problem. Finally, a derivation of the so-called Fokker-Planck equation (see equation (2.14) below) based on (2.7) is given in Appendix B.

\subsection{Fokker-Planck equation}

The following 4 sections are mainly of methodological nature without much new physics. After introducing the Fokker-Planck equation in the present section, we turn in sections 2.3 and 2.4 to the evaluation of the particle current $\langle\dot{x}\rangle$, with the result that even when the spatial symmetry is broken by the ratchet potential $V(x)$, there arises no systematic preferential motion of the random dynamics in one or the other direction. Finally, in section 2.5 the effect of an additional static "tilting"-force $F$ in the Smoluchowski-Feynman ratchet dynamics (2.6) is considered, with the expected result of a finite particle current $\langle\dot{x}\rangle$ with the same sign as the applied force $F$. Readers which are already familiar with or not interested in these standard techniques are recommended to continue with section 2.6.

Returning to (2.6), a quite natural next step is to consider a statistical ensemble of these stochastic processes belonging to independent realizations of the random fluctuations $\xi(t)$. The corresponding probability density $P(x, t)$ in space $x$ at time $t$ describes the distribution of the Brownian particles and follows as an ensemble averagef of the form

$$
P(x, t):=\langle\delta(x-x(t))\rangle .
$$

An immediate consequence of this definition is the normalization

$$
\int_{-\infty}^{\infty} d x P(x, t)=1
$$

Another trivial consequence is that $P(x, t) \geq 0$ for all $x$ and $t$.

In order to determine the time-evolution of $P(x, t)$, we first consider in (2.6) the special case $V^{\prime}(x) \equiv 0$. As discussed in detail in section A.3 of Appendix A, we are thus

\footnotetext{
${ }^{4}$ To be precise, an average over the initial conditions $x\left(t_{0}\right)$ according to some prescribed statistical weight $P\left(x, t_{0}\right)$ together with an average over the noise is understood on the right hand side of 2.9 .
} 
dealing with the force-free thermal diffusion of a Brownian particle with a diffusion coefficient $D$ that satisfies Einstein's relation 77

$$
D=k_{B} T / \eta
$$

Consequently, $P(x, t)$ is governed by the diffusion equation

$$
\frac{\partial}{\partial t} P(x, t)=\frac{k_{B} T}{\eta} \frac{\partial^{2}}{\partial x^{2}} P(x, t) \quad \text { if } \quad V^{\prime}(x) \equiv 0 .
$$

Next we address the deterministic dynamics $\xi(t) \equiv 0$ in (2.6). In complete analogy to classical Hamiltonian mechanics, one then finds that the probability density $P(x, t)$ evolves according to a Liouville-equation of the form?

$$
\frac{\partial}{\partial t} P(x, t)=\frac{\partial}{\partial x}\left\{\frac{V^{\prime}(x)}{\eta} P(x, t)\right\} \quad \text { if } \quad \xi(t) \equiv 0 .
$$

Since both (2.12) and (2.13) are linear in $P(x, t)$ it is quite obvious that the general case follows by combination of both contributions, i.e., one obtains the so-called FokkerPlanck equation 99, 101,

$$
\frac{\partial}{\partial t} P(x, t)=\frac{\partial}{\partial x}\left\{\frac{V^{\prime}(x)}{\eta} P(x, t)\right\}+\frac{k_{B} T}{\eta} \frac{\partial^{2}}{\partial x^{2}} P(x, t)
$$

where the first term on the right hand side is called "drift term" and the second "diffusion term".

While our above derivation of the Fokker-Planck equation is admittedly of a rather heuristic nature, it is appealing due to its extreme simplicity and the intuitive physical way of reasoning. A more rigorous calculation, based on the discretized dynamics (2.7) in the limit $\Delta t \rightarrow 0$ is provided in Appendix B. Numerous alternative derivations can be found e.g. in 98 105 and further references therein. A brief historical account of the Fokker-Planck equation has been compiled in [106], see also [107].

\subsection{Particle current}

The quantity of foremost interest in the context of transport in periodic systems is the particle current $\langle\dot{x}\rangle$, defined as the time-dependent ensemble average over the velocities

$$
\langle\dot{x}\rangle:=\langle\dot{x}(t)\rangle \text {. }
$$

For later convenience, the argument $t$ in $\langle\dot{x}\rangle$ is omitted. Obviously, the probability density $P(x, t)$ contains the entire information about the system; in this section we treat the question of how to extract the current $\langle\dot{x}\rangle$ out of it.

The simplest way to establish such a connection between $\langle\dot{x}\rangle$ and $P(x, t)$ follows by averaging in (2.6) and taking into account (2.4), i.e., $\langle\dot{x}\rangle=-\left\langle V^{\prime}(x(t))\right\rangle / \eta$. Since

\footnotetext{
${ }^{5}$ Proof: Let $x(t)$ be a solution of $\dot{x}(t)=f(x(t))$ and define $P(x, t):=\delta(x-x(t))$. Note that the variable $x$ and the function $x(t)$ are mathematically completely unrelated objects. Then $\frac{\partial}{\partial t} P(x, t)$ $=-\dot{x}(t) \frac{\partial}{\partial x} \delta(x-x(t))=-f(x(t)) \frac{\partial}{\partial x} \delta(x-x(t))=-\frac{\partial}{\partial x}\{f(x(t)) \delta(x-x(t))\}=-\frac{\partial}{\partial x}\{f(x) \delta(x-x(t))\}$ (the last identity can be verified by operating with $\int d x h(x)$ on both sides, where $h(x)$ is an arbitrary test function with $h(x \rightarrow \pm \infty)=0$, and then performing a partial integration). Thus 2.13 is recovered for a $\delta$-distributed initial condition. Since this equation 2.13) is linear in $P(x, t)$, the case of a general initial distribution follows by linear superposition.
} 
the ensemble average means by definition an average with respect to the probability density $P(x, t)$ we arrive at our first basic observation, namely the connection between $\langle\dot{x}\rangle$ and $P(x, t)$ :

$$
\langle\dot{x}\rangle=-\int_{-\infty}^{\infty} d x \frac{V^{\prime}(x)}{\eta} P(x, t)
$$

The above derivation of (2.16) has the disadvantage that the specific form (2.6) of the stochastic dynamics has been exploited. For later use, we next sketch an alternative, more general derivation: From the definition (2.9) one obtains, independently of any details of the dynamics governing $x(t)$, a so-called master equation 99 101

$$
\begin{aligned}
& \frac{\partial}{\partial t} P(x, t)+\frac{\partial}{\partial x} J(x, t)=0 \\
& J(x, t):=\langle\dot{x}(t) \delta(x-x(t))\rangle .
\end{aligned}
$$

Note that the symbols $x$ and $x(t)$ denote here completely unrelated mathematical objects. The master equation (2.17) has the form of a continuity equation for the probability density associated with the conservation of particles, hence $J(x, t)$ is called the probability current. Upon integrating (2.18), the following completely general connection between the probability current and the particle current is obtained:

$$
\langle\dot{x}\rangle=\int_{-\infty}^{\infty} d x J(x, t) .
$$

By means of a partial integration, the current in (2.19) can be rewritten as $-\int d x x \partial J(x, t) / \partial x$ and by exploiting (2.17) one recovers the relation

$$
\langle\dot{x}\rangle=\frac{d}{d t} \int_{-\infty}^{\infty} d x x P(x, t)
$$

which may thus be considered as an alternative definition of the particle current $\langle\dot{x}\rangle$.

For the specific stochastic dynamics (2.6), we find by comparison of the FokkerPlanck equation (2.14) with the general master equation (2.17) the explicit expression for the probability current

$$
J(x, t)=-\left\{\frac{V^{\prime}(x)}{\eta}+\frac{k_{B} T}{\eta} \frac{\partial}{\partial x}\right\} P(x, t)
$$

up to an additive, $x$-independent function. Since both, $J(x, t)$ and $P(x, t)$ approach zero for $x \rightarrow \pm \infty$, it follows that this function must be identically zero. By introducing (2.21) into (2.19) we finally recover (2.16).

\subsection{Solution and discussion}

Having established the evolution equation (2.14) governing the probability density $P(x, t)$ our next goal is to actually solve it and determine the current $\langle\dot{x}\rangle$ according to (2.19). Such a calculation is illustrated in detail in this section. 
We start with introducing the so-called reduced probability density and reduced probability current

$$
\begin{aligned}
\hat{P}(x, t) & :=\sum_{n=-\infty}^{\infty} P(x+n L, t) \\
\hat{J}(x, t) & :=\sum_{n=-\infty}^{\infty} J(x+n L, t) .
\end{aligned}
$$

Taking into account (2.10), (2.19) it follows that

$$
\begin{aligned}
& \hat{P}(x+L, t)=\hat{P}(x, t) \\
& \int_{0}^{L} d x \hat{P}(x, t)=1 \\
& \langle\dot{x}\rangle=\int_{0}^{L} d x \hat{J}(x, t) .
\end{aligned}
$$

With $P(x, t)$ being a solution of the Fokker-Planck equation (2.14) it follows with (2.2) that also $P(x+n L, t)$ is a solution for any integer $n$. Since the Fokker-Planck equation is linear, it is also satisfied by the reduced density (2.22). With (2.21) it can furthermore be recast into the form of a continuity equation:

$$
\frac{\partial}{\partial t} \hat{P}(x, t)+\frac{\partial}{\partial x} \hat{J}(x, t)=0
$$

with the explicit form of the reduced probability current

$$
\hat{J}(x, t)=-\left\{\frac{V^{\prime}(x)}{\eta}+\frac{k_{B} T}{\eta} \frac{\partial}{\partial x}\right\} \hat{P}(x, t) .
$$

In other words, as far as the particle current $\langle\dot{x}\rangle$ is concerned, it suffices to solve the Fokker-Planck equation with periodic boundary (and initial) conditions.

An interesting counterpart of $(2.20)$ arises by operating with $\int_{x_{0}}^{x_{0}+L} d x x \ldots$ on both sides of (2.27), namely

$$
\langle\dot{x}\rangle=\frac{d}{d t}\left[\int_{x_{0}}^{x_{0}+L} d x x \hat{P}(x, t)\right]+L \hat{J}\left(x_{0}, t\right)
$$

where $x_{0}$ is an arbitrary reference position. In other words, the total particle current $\langle\dot{x}\rangle$ is composed of the motion of the "center of mass" $\int_{x_{0}}^{x_{0}+L} d x x \hat{P}(x, t)$ plus $L$ times the reduced probability current $\hat{J}\left(x_{0}, t\right)$ at the reference point $x_{0}$. Especially, if the reduced dynamics assumes a steady state, characterized by $d \hat{P}(x, t) / d t=0$, then the reduced probability current $\hat{J}\left(x_{0}, t\right)=\hat{J}^{s t}$ becomes independent of $x_{0}$ and $t$ according to (2.27), (2.28), and the particle current takes the suggestive form

$$
\langle\dot{x}\rangle=L \hat{J}^{s t} .
$$

We recall that in general the current $\langle\dot{x}\rangle$ is time dependent but the argument $t$ is omitted (cf. (2.15)). However, the most interesting case is usually its behavior in the long-time limit, corresponding to a steady state in the reduced description (unless an 
external driving prohibits its existence, see e.g. section 2.6.1 below). In this case, no implicit $t$-dependent of $\langle\dot{x}\rangle$ is present any more, see (2.30).

We have tacitly assumed here that the original problem (2.6) extends over the entire real $x$-axis. In some cases, a periodicity condition after one or several periods $L$ of the potential $V(x)$ may represent a more natural modeling, for instance in the original Smoluchowski-Feynman ratchet of circular shape (figure 2.1). One readily sees, that in such a case (2.24)-(2.30) remain valid without any change. We furthermore remark that the specific form of the stochastic dynamics (2.6) or of the equivalent master equation (2.17), (2.21) has only been used in (2.28), while equations (2.22)-(2.27), (2.29), (2.30) remain valid for more general stochastic dynamics.

For physical reasons we expect that the reduced probability density $\hat{P}(x, t)$ indeed approaches a steady state $\hat{P}^{s t}(x)$ in the long-time limit $t \rightarrow \infty$ and hence $\hat{J}\left(x_{0}, t\right) \rightarrow$ $\hat{J}^{s t}$. From the remaining ordinary first order differential equation (2.28) for $P^{s t}(x)$ in combination with (2.24) it follows that $\hat{J}^{s t}$ must be zero and therefore the solution is

$$
\begin{aligned}
& \hat{P}^{s t}(x)=Z^{-1} e^{-V(x) / k_{B} T} \\
& Z:=\int_{0}^{L} d x e^{-V(x) / k_{B} T}
\end{aligned}
$$

while (2.26) implies for the steady state particle current the result

$$
\langle\dot{x}\rangle=0
$$

It can be shown that the long-time asymptotics of a Fokker-Planck equation like in (2.27), (2.28) is unique 82, 83, 100, 108, 109]. Consequently, this unique solution must be (2.31), independent of the initial conditions. Furthermore, our assumption that a steady state is approached for $t \rightarrow \infty$ is self-consistently confirmed.

The above results justify a posteriori our proposition that (2.6) models an overdamped Brownian motion under the influence of a thermal equilibrium heat bath at temperature $T$ : indeed, in the long time limit (steady state), equation (2.31) correctly reproduces the expected Boltzmann distribution and the average particle current vanishes (2.33), as required by the second law of thermodynamics. The importance of such consistency checks when modeling thermal noise is further discussed in section 2.9 .

Much like in the original ratchet and pawl gadget (figure 2.1), the absence of an average current (2.33) is on the one hand a simple consequence of the second law of thermodynamics. On the other hand, when looking at the stochastic motion in a ratchet-shaped potential like in figure 2.2, it is nevertheless quite astonishing that in spite of the broken spatial symmetry there arises no systematic preferential motion of the random dynamics in one or the other direction.

Note that if the original problem (2.6) extends over the entire real axis (bringing along natural boundary conditions), then the probability density $P(x, t)$ will never approach a meaningful steady state. It is only the reduced density $\hat{P}(x, t)$, associated with periodic boundary conditions, which tends toward a meaningful $t$-independent long-time limit. In particular, only after this mapping, which leaves the particle current unchanged, are the concepts of equilibrium statistical mechanics applicable.

\footnotetext{
${ }^{6}$ The trivial long time behavior $P(x, t) \rightarrow 0$ does not admit any further conclusions and is therefore not considered as a meaningful steady state.
} 
Conceptionally, the simplified Smoluchowski-Feynman ratchet model (2.6) has one crucial advantage in comparison with the original full-blown ratchet and pawl gadget from figure 2.1: The second law of thermodynamics has not to be invoked as a kind of deus ex machina, rather the absence of a current (2.33) now follows directly from the basic model (2.6), without any additional assumptions. As a consequence, modifications of the original situation, for which the second law of thermodynamics no longer applies, are relatively straightforward to treat within a correspondingly modified Smoluchowski-Feynman ratchet model (2.6), but become very cumbersome [110, 111] for the more complicated original ratchet and pawl gadget from figure 2.1. A first, very simple such modification of the Smoluchowski-Feynman ratchet model will be addressed next.

\subsection{Tilted Smoluchowski-Feynman ratchet}

In this section we consider the generalization of the overdamped SmoluchowskiFeynman ratchet model (2.6) in the presence of an additional homogeneous, static force $F$ :

$$
\eta \dot{x}(t)=-V^{\prime}(x(t))+F+\xi(t)
$$

This scenario represents a kind of "hydrogen atom" in that it is one of the few exactly solvable cases and will furthermore turn out to be equivalent to the archetypal ratchet models considered later in sections 4.3.2, 4.4.1, 5.0.3, 6.1, and 9.2. For instance, in the original ratchet and pawl gadget (figure 2.1) such a force $F$ in (2.34) models the effect of a constant external torque due to a load.

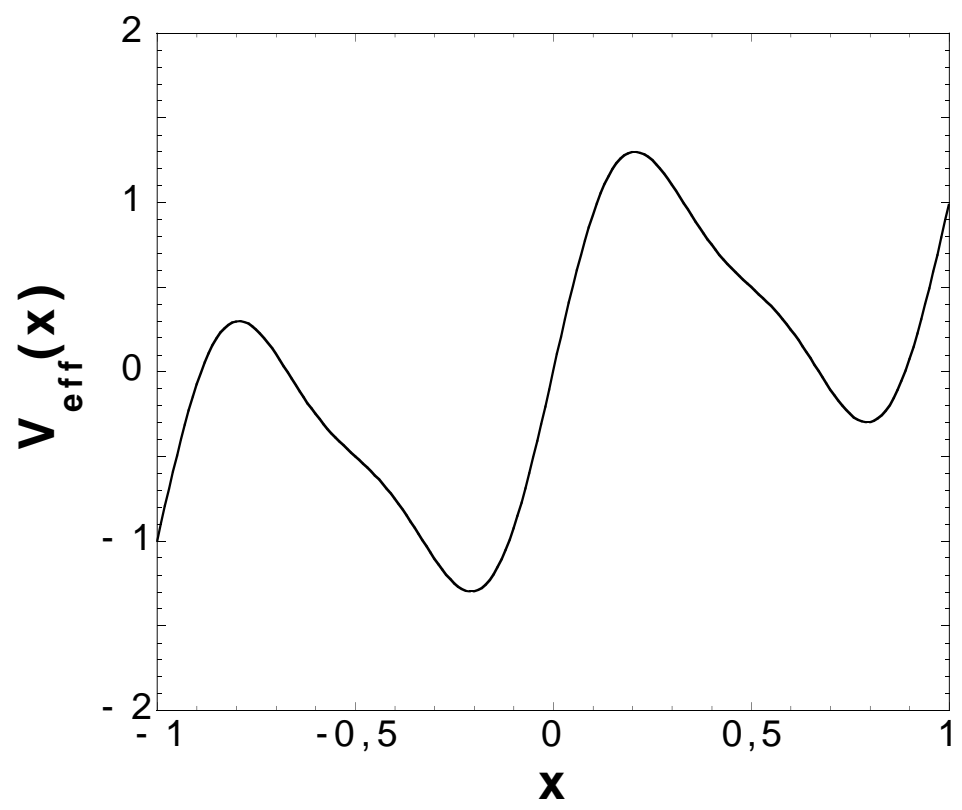

Figure 2.3: Typical example of an effective potential from (2.35) "tilted to the left", i.e. $F<0$. Plotted is the example from (2.3) in dimensionless units (see section A.4 in Appendix A) with $L=V_{0}=1$ and $F=-1$, i.e. $V_{\text {eff }}(x)=\sin (2 \pi x)+0.25 \sin (4 \pi x)+x$. 
We may incorporate the ratchet potential $V(x)$ and the force $F$ into a single effective potential

$$
V_{\text {eff }}(x):=V(x)-x F
$$

which the Brownian particle (2.34) experiences. E.g. for a negative force $F<0$, pulling the particles to the left, the effective potential will be tilted to the left as well, see figure 2.3. In view of $\langle\dot{x}\rangle=0$ for $F=0$ (see previous section) it is plausible that in such a potential the particles will move on the average "downhill", i.e., $\langle\dot{x}\rangle<0$ for $F<0$ and similarly $\langle\dot{x}\rangle>0$ for $F>0$. This surmise is confirmed by a detailed calculation along the very same lines as for $F=0$ (see section 2.4), with the result (see 112 114 and also Vol.2, Chapter 9 in 115) that in the steady state (long time limit)

$$
\begin{aligned}
& \hat{P}^{s t}(x)=\mathcal{N} \frac{\eta}{k_{B} T} e^{-V_{\text {eff }}(x) / k_{B} T} \int_{x}^{x+L} d y e^{V_{\text {eff }}(y) / k_{B} T} \\
& \langle\dot{x}\rangle=L \mathcal{N}\left[1-e^{\left[V_{\text {eff }}(L)-V_{\text {eff }}(0)\right] / k_{B} T}\right] \\
& \mathcal{N}:=\frac{k_{B} T}{\eta}\left[\int_{0}^{L} d x \int_{x}^{x+L} d y e^{\left[V_{\text {eff }}(y)-V_{\text {eff }}(x)\right] / k_{B} T}\right]^{-1} .
\end{aligned}
$$

Note that for the specific form (2.35) of the effective potential we can further simplify $(2.37)$ by exploiting that $V_{\text {eff }}(L)-V_{\text {eff }}(0)=-L F$. However, the result (2.36)(2.38) is valid for completely general effective potentials $V_{\text {eff }}^{\prime}(x)$ provided $V_{\text {eff }}^{\prime}(x+L)=$ $V_{\text {eff }}^{\prime}(x)$.

Our first observation is that a time independent probability density $\hat{P}^{s t}(x)$ does not exclude a non-vanishing particle current $\langle\dot{x}\rangle$. Exploiting (2.35), one readily sees that as expected - the sign of this current (2.37) agrees with the sign of $F$. Furthermore one can prove that the current is a monotonically increasing function of $F$ [116 and that for any fixed $F$-value, the current is maximal (in modulus) when $V(x)=$ const. (see section 4.4.1). The typical quantitative behavior of the steady state current (2.37) as a function of the applied force $F$ (called "response curve", "load curve", or (currentforce-) "characteristics") is exemplified in figure 2.4. Note that the leading order ("linear response") behavior is symmetric about the origin, but not the higher order contributions.

The occurrence of a non-vanishing particle current in (2.37) signals that 2.36 describes a steady state which is not in thermal equilibrium, and actually far from equilibrium unless $F$ is very small 7 . As mentioned already at the end of the previous section, while at (and near) equilibrium one may question the need of a microscopic model like in (2.34) in view of the powerful principles of equilibrium statistical mechanics, such an approach has the advantage of remaining valid far from equilibrium\&, where no such general statistical mechanical principles are available.

\footnotetext{
${ }^{7}$ In particular, the effective diffusion coefficient is no longer related to the mobility via a generalized Einstein relation 2.11, i.e. $D_{\text {eff }}=k_{B} T \partial\langle\dot{x}\rangle / \partial F$ only holds for $F=0$ [117.

${ }^{8}$ Note that there is no inconsistency of a thermal (white) noise $\xi(t)$ appearing in a system far from thermal equilibrium: any system (equilibrium or not) can be in contact with a thermal heat bath.
} 


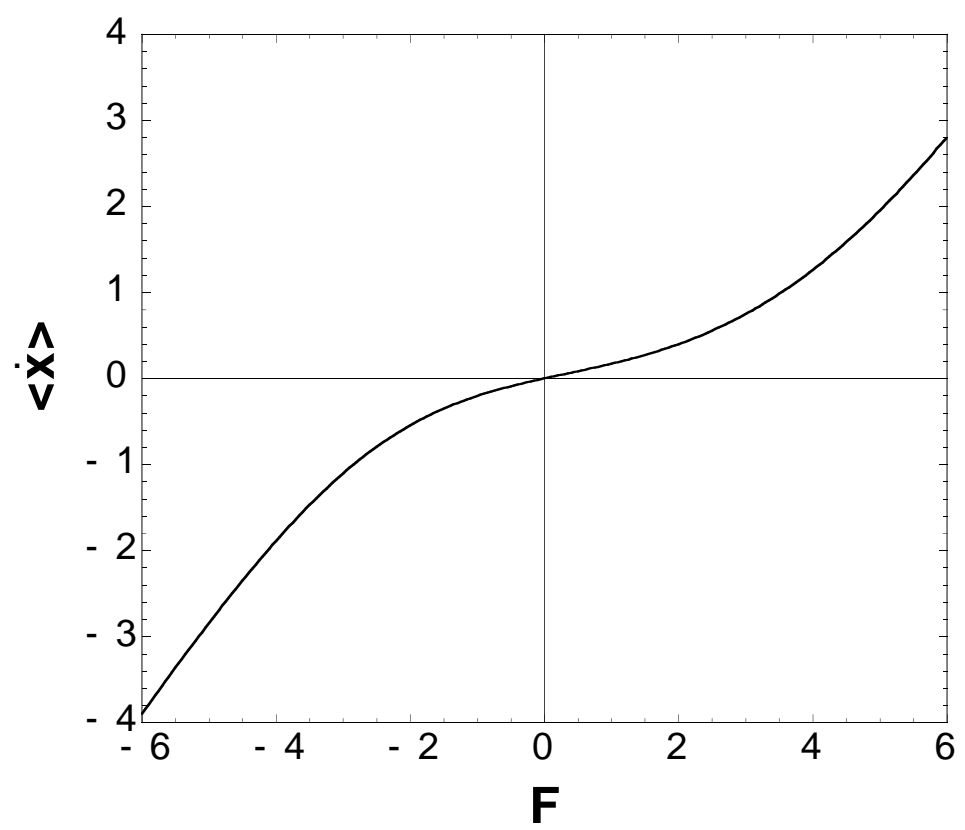

Figure 2.4: $\quad$ Steady state current $\langle\dot{x}\rangle$ from (2.37) versus force $F$ for the tilted Smoluchowski-Feynman ratchet dynamics (2.5), (2.34) with the potential (2.3) in dimensionless units (see section A.4 in Appendix A) with $\eta=L=V_{0}=k_{B}=1$ and $T=0.5$. Note the broken point-symmetry.

As pointed out at the end of the preceding section, only the reduced probability density $\hat{P}(x, t)$ approaches a meaningful steady state, but not the original dynamics (2.34), extending over the entire $x$-axis. Thus, stability criteria for steady states, both mechanical and thermodynamical, can only be discussed in the former, reduced setup. As compared to the usual reflecting boundary conditions in this context, the present periodic boundary conditions entail some quite unusual consequences: With the definition $\mu\left(F, x_{0}\right):=\int_{x_{0}}^{x_{0}+L} d x x \hat{P}^{s t}(x)$ for the "center of mass" in the steady state (cf. (2.29) ), one can infer from the periodicity $\hat{P}^{s t}(x+L)=\hat{P}^{s t}(x)$ and the normalization $\int_{0}^{L} d x \partial \hat{P}^{s t}(x) / \partial F=0$ that $\partial \mu\left(F, x_{0}+L\right) / \partial F=\partial \mu\left(F, x_{0}\right) / \partial F$, where $x_{0}$ is an arbitrary reference position. Furthermore, one finds that

$$
\int_{0}^{L} d x_{0} \frac{\partial \mu\left(F, x_{0}\right)}{\partial F}=\int_{0}^{L} d x_{0} \int_{0}^{L} d x\left(x+x_{0}\right) \frac{\partial \hat{P}^{s t}\left(x+x_{0}\right)}{\partial F}=0
$$

Excluding the non-generic case that $\partial \mu\left(F, x_{0}\right) / \partial F$ is identically zero, it follows 5 that $\partial \mu\left(F, x_{0}\right) / \partial F$ may be negative or positive, depending on the choice of $x_{0}$. In other words, the "center of mass" may move either in the same or in the opposite direction of the applied force $F$, and this even if the unperturbed system is at thermal equilibrium. Similarly, also with respect to the dependence of the steady state current $\langle\dot{x}\rangle$ upon the applied force $F$, no general a priori restrictions due to certain "stability criteria" for steady states exist.

\footnotetext{
${ }^{9}$ Note that we did not exploit any specific property of the underlying stochastic dynamics.
} 


\subsubsection{Weak noise limit}

In this section we work out the simplification of the current-formula (2.37) for small thermal energies $k_{B} T$ - see equation (2.44) below - and its quite interesting physical interpretation, repeatedly re-appearing later on.

Focusing on not too large $F$-values, such that $V_{\text {eff }}(x)$ in (2.35) still exhibits at least one local minimum and maximum within each period $L$, one readily sees that the function $V_{\text {eff }}(y)-V_{\text {eff }}(x)$ has generically a unique global maximum within the two-dimensional integration domain in (2.38), say at the point $(x, y)=\left(x_{\min }, x_{\max }\right)$, where $x_{\min }$ is a local minimum of $V_{\text {eff }}(x)$ and $x_{\max }$ a local maximum, sometimes called metastable and activated states, respectively. Within $\left(x_{\min }, x_{\min }+L\right)$ the point $x_{\max }$ is moreover a global maximum of $V_{\text {eff }}(x)$ and similarly $x_{\min }$ a global minimum within $\left(x_{\max }-L, x_{\max }\right)$, i.e.

$$
\Delta V_{\text {eff }}:=V_{\text {eff }}\left(x_{\max }\right)-V_{\text {eff }}\left(x_{\min }\right)
$$

is the effective potential barrier that the particle has to surmount in order to proceed from the metastable state $x_{\min }$ to $x_{\min }+L$. Likewise,

$$
V_{\mathrm{eff}}\left(x_{\max }-L\right)-V_{\mathrm{eff}}\left(x_{\mathrm{min}}\right)=\Delta V_{\mathrm{eff}}-\left[V_{\mathrm{eff}}(L)-V_{\mathrm{eff}}(0)\right]
$$

is the barrier between $x_{\min }$ and $x_{\min }-L$. For small thermal energies

$$
k_{B} T \ll\left\{\Delta V_{\text {eff }}, \Delta V_{\text {eff }}-\left[V_{\text {eff }}(L)-V_{\text {eff }}(0)\right]\right\}
$$

the main contribution in (2.38) stems from a small vicinity of the absolute maximum $\left(x_{\min }, x_{\max }\right)$ and we thus can employ the so-called saddle point approximation

$$
V_{\mathrm{eff}}(y)-V_{\mathrm{eff}}(x) \simeq \Delta V_{\mathrm{eff}}-\frac{\left|V_{\mathrm{eff}}^{\prime \prime}\left(x_{\mathrm{max}}\right)\right|}{2}\left(y-x_{\mathrm{max}}\right)^{2}-\frac{\left|V_{\mathrm{eff}}^{\prime \prime}\left(x_{\mathrm{min}}\right)\right|}{2}\left(x-x_{\mathrm{min}}\right)^{2},
$$

where we have used that $V_{\text {eff }}^{\prime}\left(x_{\max }\right)=V_{\text {eff }}^{\prime}\left(x_{\min }\right)=0$ and $V_{\text {eff }}^{\prime \prime}\left(x_{\max }\right)<0, V_{\text {eff }}^{\prime \prime}\left(x_{\min }\right)>0$. Within the same approximation, the two integrals in (2.38) can now be extended over the entire real $x$ - and $y$-axis. Performing the two remaining Gaussian integrals in 2.38) yields for the current (2.37) the result

$$
\begin{aligned}
\langle\dot{x}\rangle & =L\left[k_{+}-k_{-}\right] \\
k_{+} & :=\frac{\left|V_{\mathrm{eff}}^{\prime \prime}\left(x_{\mathrm{max}}\right) V_{\mathrm{eff}}^{\prime \prime}\left(x_{\mathrm{min}}\right)\right|^{1 / 2}}{2 \pi \eta} e^{-\Delta V_{\mathrm{eff}} / k_{B} T} \\
k_{-} & :=k_{+} e^{\left[V_{\mathrm{eff}}(L)-V_{\mathrm{eff}}(0)\right] / k_{B} T} \\
& =\frac{\left|V_{\mathrm{eff}}^{\prime \prime}\left(x_{\max }-L\right) V_{\mathrm{eff}}^{\prime \prime}\left(x_{\min }\right)\right|^{1 / 2}}{2 \pi \eta} e^{-\left[V_{\mathrm{eff}}\left(x_{\max }-L\right)-V_{\mathrm{eff}}\left(x_{\min }\right)\right] / k_{B} T},
\end{aligned}
$$

where we have exploited (2.41) and the periodicity of $V_{\text {eff }}^{\prime \prime}(x)$ in the last relation in (2.46).

One readily sees that $k_{+}$is identical to the so-called Kramers-Smoluchowski rate 66] for transitions from $x_{\min }$ to $x_{\min }+L$, and similarly $k_{-}$is the escape rate from $x_{\min }$ to $x_{\min }-L$. For weak thermal noise (2.42) these rates are small and the current 2.44) takes the suggestive form of a net transition frequency (rate to the right minus rate to the left) between adjacent local minima of $V_{\text {eff }}(x)$ times the step size $L$ of one such transition. 


\subsection{Temperature ratchet and ratchet effect}

We now come to the central issue of the present chapter, namely the phenomenon of directed transport in a spatially periodic, asymmetric system away from equilibrium. This so-called ratchet-effect is very often illustrated by invoking as an example the onoff ratchet model, as introduced by Bug and Berne [32] and by Ajdari and Prost [34], see section 4.2. Here, we will employ a different example, the so-called temperature ratchet, which in the end will however turn out to be actually very closely related to the on-off ratchet model (see section 6.3). We emphasize that the choice of this example is not primarily based on its objective or historical significance but rather on the author's personal taste and research activities. Moreover, this example appears to be particularly suitable for the purpose of illustrating besides the ratchet effect per se also many other important concepts (see sections 2.6.3 2.11) that we will encounter again in much more generality in later chapters.

\subsubsection{Model}

As an obvious generalization of the tilted Smoluchowski-Feynman ratchet model (2.34) we consider the case that the temperature of the Gaussian white noise $\xi(t)$ in (2.5) is subjected to periodic temporal variations with period $\mathcal{T}$ [118, i.e.

$$
\begin{aligned}
& \langle\xi(t) \xi(s)\rangle=2 \eta k_{B} T(t) \delta(t-s) \\
& T(t)=T(t+\mathcal{T})
\end{aligned}
$$

where $T(t) \geq 0$ for all $t$ is taken for granted. Note that due to the time-dependent temperature in (2.47) the noise $\xi(t)$ is strictly speaking no longer stationary. A stationary noise is, however, readily recovered by rewriting (2.34), (2.47) as

$$
\eta \dot{x}(t)=-V^{\prime}(x(t))+F+g(t) \hat{\xi}(t)
$$

where $\hat{\xi}(t)$ is a Gaussian white noise with $\langle\hat{\xi}(t) \hat{\xi}(s)\rangle=2 \delta(t-s)$ and $g(t):=\left[\eta k_{B} T(t)\right]^{1 / 2}$. Two typical examples which we will adopt for our numerical explorations below are

$$
\begin{aligned}
T(t) & =\bar{T}[1+A \operatorname{sign}\{\sin (2 \pi t / \mathcal{T})\}] \\
T(t) & =\bar{T}[1+A \sin (2 \pi t / \mathcal{T})]^{2}
\end{aligned}
$$

where $\operatorname{sign}(x)$ denotes the signum function and $|A|<1$. The first example (2.50) thus jumps between $T(t)=\bar{T}[1+A]$ and $T(t)=\bar{T}[1-A]$ at every half period $\mathcal{T} / 2$. The motivation for the square on the right hand side of (2.51) becomes apparent when rewriting the dynamics in the form (2.49).

Similarly as in section 2.2, one finds that the reduced particle density (2.22) for this so-called temperature ratchet model (2.34), (2.47), (2.48) is governed by the FokkerPlanck equation

$$
\frac{\partial}{\partial t} \hat{P}(x, t)=\frac{\partial}{\partial x}\left\{\frac{V^{\prime}(x)-F}{\eta} \hat{P}(x, t)\right\}+\frac{k_{B} T(t)}{\eta} \frac{\partial^{2}}{\partial x^{2}} \hat{P}(x, t) .
$$

Due to the permanent oscillations of $T(t)$, this equation does not admit a timeindependent solution. Hence, the reduced density $\hat{P}(x, t)$ will not approach a steady 
state but rather a unique periodic behavior in the long time limit natural to include a time average into the definition (2.15) of the particle current. Keeping for convenience the same symbol $\langle\dot{x}\rangle$, the generalized expression (2.26), (2.28) for this current becomes

$$
\langle\dot{x}\rangle=\frac{1}{\mathcal{T}} \int_{t}^{t+\mathcal{T}} d t \int_{0}^{L} d x \frac{F-V^{\prime}(x)}{\eta} \hat{P}(x, t) .
$$

Note that in general, the current $\langle\dot{x}\rangle$ in (2.53) is still $t$-dependent. Only in the long time limit, corresponding in the reduced description to a $\mathcal{T}$-periodic $\hat{P}(x, t)$, this $t$-dependence disappears. Usually, this latter long-time limit is of foremost interest.

\subsubsection{Ratchet effect}

After these technical preliminaries, we return to the physics of our model (2.34), 2.47), (2.48): In the case of the tilted Smoluchowski-Feynman ratchet (time-independent temperature $T$ ), equation (2.37) tells us that for a given force, say $F<0$, the particle will move "downhill" on the average, i.e. $\langle\dot{x}\rangle<0$, and this for any fixed (positive) value of the temperature $T$. Turning to the temperature ratchet with $T$ being now subjected to periodic temporal variations, one therefore should expect that the particles still move "downhill" on the average. The numerically calculated "load curve" in figure 2.5 demonstrates that the opposite is true within an entire interval of negative $F$ values. Surprisingly indeed, the particles are climbing "uphill" on the average, thereby performing work against the load force $F$, which apparently can have no other origin than the white thermal noise $\xi(t)$.

A conversion (rectification) of random fluctuations into useful work as exemplified above is called "ratchet effect". For a setup of this type, the names thermal ratchet [17, 10, 11], Brownian motor [48, 118], Brownian rectifier [51] (mechanical diode 11]), stochastic ratchet [119, 120], or simply ratchet are in use 1 . Since the average particle current $\langle\dot{x}\rangle$ usually depends continuously on the load force $F$, it is for a qualitative analysis sufficient to consider the case $F=0$ : the occurrence of the ratchet effect is then tantamount to a finite current

$$
\langle\dot{x}\rangle \neq 0 \quad \text { for } \quad F=0
$$

i.e. the unbiased Brownian motor implements a "particle pump". The necessary force $F$ which leads to an exact cancellation of the ratchet effects, i.e $\langle\dot{x}\rangle=0$, is called the "stopping force". The property (2.54) is the distinguishing feature between the ratchet effect and the somewhat related so-called negative mobility effect, encountered later in section 9.2.4.

\footnotetext{
${ }^{10}$ Proof: Since $T(t+\mathcal{T})=T(t)$ we see that with $\hat{P}(x, t)$ also $\hat{P}(x, t+\mathcal{T})$ solves $(2.52)$. Moreover, for the long time asymptotics of 2.52 the general proof of uniqueness from 83,109 applies. Consequently, $\hat{P}(x, t+\mathcal{T})$ must converge towards $\hat{P}(x, t)$, i.e. $\hat{P}(x, t)$ is periodic and unique for $t \rightarrow \infty$.

${ }^{11}$ The notion "molecular motor" should be reserved for models focusing specifically on intracellular transport processes, see chapter 6 . Similarly, the notion "Brownian ratchet" has been introduced in a rather differen context, namely as a possible operating principle for the translocation of proteins accross membranes 121.25 .
} 


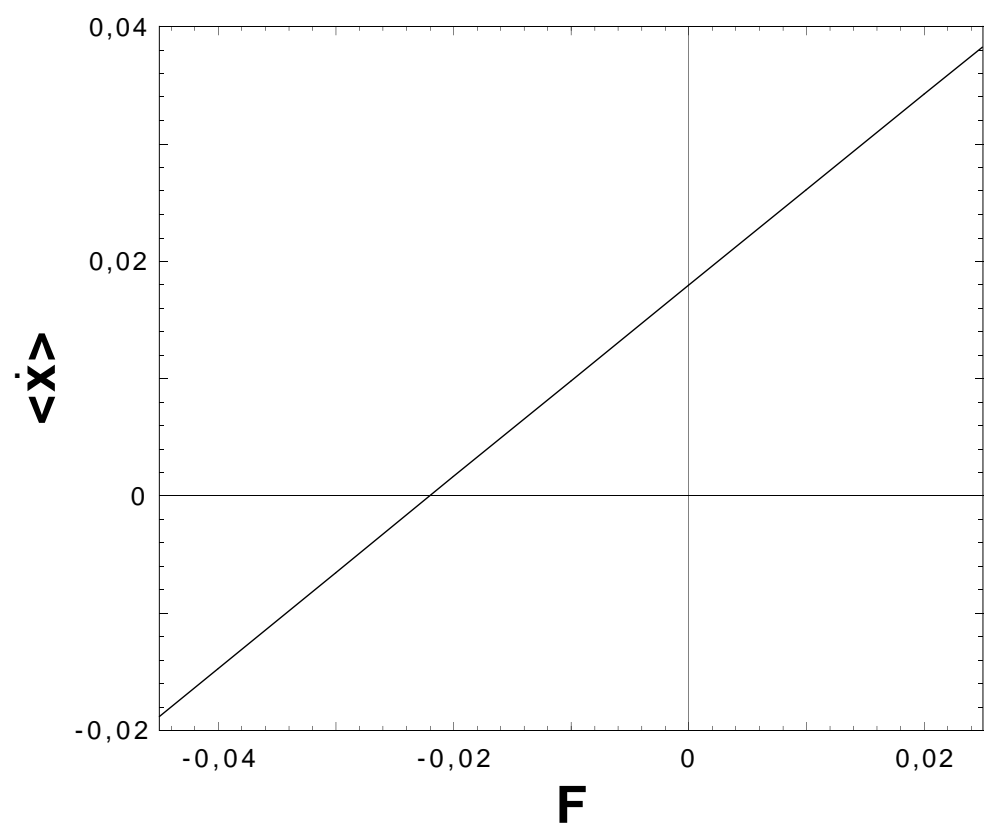

Figure 2.5: Average particle current $\langle\dot{x}\rangle$ versus force $F$ for the temperature ratchet dynamics (2.3), (2.34), 2.47), (2.50) in dimensionless units (see section A.4 in Appendix A). Parameter values are $\eta=L=\mathcal{T}=k_{B}=1, V_{0}=1 / 2 \pi, \bar{T}=0.5, A=0.8$. The time- and ensemble averaged current (2.53) has been obtained by numerically evolving the Fokker-Planck equation (2.52) until transients have died out.

\subsubsection{Discussion}

In order to understand the basic physical mechanism behind the ratchet effect at $F=0$, we focus on the dichotomous periodic temperature modulations from (2.50). During a first time interval, say $t \in[\mathcal{T} / 2, \mathcal{T}]$, the thermal energy $k_{B} T(t)$ is kept at a constant value $\bar{T}[1-A]$ much smaller than the potential barrier $\Delta V$ between two neighboring local minima of $V(x)$. Thus, all particles will have accumulated in a close vicinity of the potential minima at the end of this time interval, as sketched in the lower panel of figure 2.6. Then the thermal energy jumps to a value $\bar{T}[1+A]$ much larger than $\Delta V$ and remains there during another half period, say $t \in[\mathcal{T}, 3 \mathcal{T} / 2]$. Since the particles then hardly feel the potential any more in comparison to the violent thermal noise, they spread out practically like in the case of free thermal diffusion (upper panel in figure 2.6). Finally, $T(t)$ jumps back to its original low value $\bar{T}[1-A]$ and the particles slide downhill towards the respective closest local minima of $V(x)$. Due to the asymmetry of the potential $V(x)$, the original population of one given minimum is re-distributed asymmetrically and a net average displacement results after one time-period $\mathcal{T}$.

In the case that the potential $V(x)$ has exactly one minimum and maximum per period $L$ (as it is the case in figure 2.6) it is quite obvious that if the local minimum is closer to its adjacent maximum to the right (as in figure 2.6), a positive particle current $\langle\dot{x}\rangle>0$ will arise, otherwise a negative current. For potentials with additional extrema, the determination of the current direction may be less obvious. 

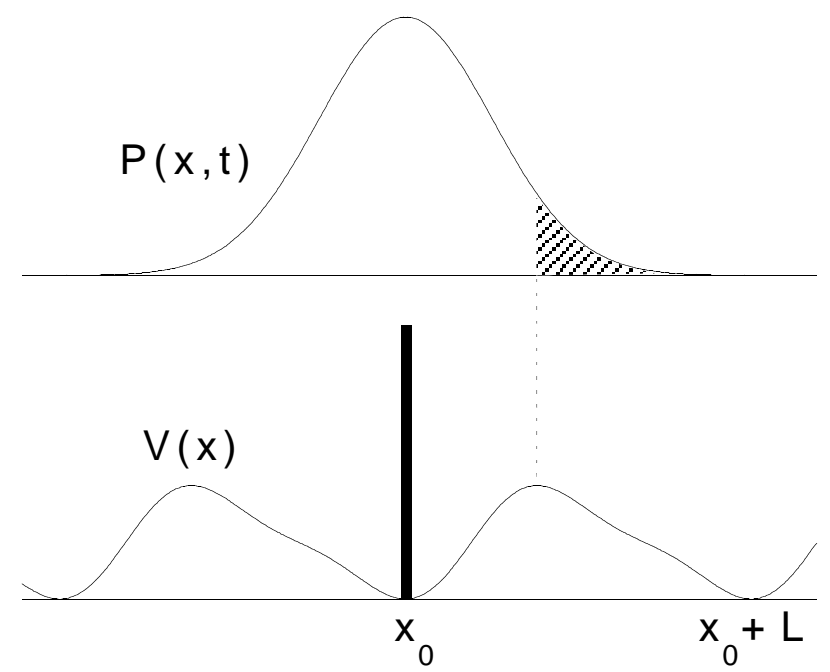

Figure 2.6: The basic working mechanism of the the temperature ratchet (2.34), (2.47), (2.50). The figure illustrates how Brownian particles, initially concentrated at $x_{0}$ (lower panel), spread out when the the temperature is switched to a very high value (upper panel). When the temperature jumps back to its initial low value, most particles get captured again in the basin of attraction of $x_{0}$, but also substantially in that of $x_{0}+L$ (hatched area). A net current of particles to the right, i.e. $\langle\dot{x}\rangle>0$ results. Note that practically the same mechanism is at work when the temperature is kept fixed and instead the potential is turned "on" and "off" (on-off ratchet, see section 4.2).

As expected, a qualitatively similar behavior is observed for more general temperature modulations $T(t)$ than in figure 2.6 provided they are sufficiently slow. The effect is furthermore robust with respect to the potential shape [118] and persists even for (slow) random instead of deterministic changes of $T(t)$ [126, 127], e.g. (rare) random flips between the two possible values in figure 2.6, as well as for a modified dynamics with a discretized state space [128, 129]. The case of finite inertia and of various correlated (colored) Gaussian noises instead of the white noise in (2.34) or (2.49) has been addressed in [130] and [131], respectively. A somewhat more detailed quantitative analysis will be given in sections 2.10 and 2.11 below.

In practice, the required magnitudes and time scales of the temperature variations may be difficult to realize experimentally by directly adding and extracting heat, but may well be feasible indirectly, e.g. by pressure variations. An exception are point contact devices with a defect which tunnels incoherently between two states and thereby changes the coupling strength of the device to its thermal environment 132 138. In other words, when incorporated into an electrical circuit, such a device exhibits random dichotomous jumps both of its electrical resistance and of the intensity of the thermal fluctuations which it produces [139]. The latter may thus be exploited to drive a temperature ratchet system [126].

Further, it has been suggested 140, 141] that microorganisms living in convective hot springs may be able to extract energy out of the permanent temperature variations they experience; the temperature ratchet is a particularly simple mechanism which 
could do the job. Moreover, a temperature ratchet-type modification of the experiment by Kelly, Tellitu, and Sestelo [73 75] (cf. section 2.1.1) has been proposed in [76].

Finally, it is known that certain enzymes (molecular motors) in living cells are able to travel along polymer filaments by hydrolyzing ATP (adenosine triphosphate). The interaction (chemical "affinity") between molecular motor and filament is spatially periodic and asymmetric, and thermal fluctuations play a significant role on these small scales. On the crudest level, hydrolyzing an ATP molecule may be viewed as converting a certain amount of chemical energy into heat, thus we recover all the essential ingredients of a temperature ratchet. Such a temperature ratchet-type model for intracellular transport has been proposed in [7]. Admittedly, modeling the molecular motor as a Brownian particle without any relevant internal degree of freedom 12 and the ATP hydrolysis as a mere production of heat is a gross oversimplification from the biochemical point of view, see chapter 7 , but may still be acceptable as a primitive sketch of the basic physics. Especially, quantitative estimates indicate [9, 142, 143] that the temperature variations (either their amplitude or their duration) within such a temperature ratchet model may not be sufficient to reproduce quantitatively the observed traveling speed of the molecular motor.

\subsection{Mechanochemical coupling}

We begin with pointing out that the ratchet effect as exemplified by the temperature ratchet model is not in contradiction with the second law of thermodynamics ${ }^{[3}$ since we may consider the changing temperature $T(t)$ as caused by several heat baths at different temperatures ${ }^{\text {ma }}$. From this viewpoint, our system is nothing else than an extremely primitive and small heat engine 12. Specifically, the example from (2.50) and figure 2.5 represents the most common case with just two equilibrium heat baths at two different temperatures. The fact that such a device can produce work is therefore not a miracle but still amazing.

At this point it is crucial to recognize that there is also one fundamental difference between the usual types of heat engines and a Brownian motor as exemplified by the temperature ratchet: To this end we first note that the two "relevant state variables" of our present system are $x(t)$ and $T(t)$. In the case of an ordinary heat engine, these state variables would always cycle through one and the same periodic sequence of events ("working strokes"). In other words, the evolutions of the state variables $x(t)$ and $T(t)$ would be tightly coupled together (interlocked, synchronized). As a consequence, a single suitably defined effective state variable would actually be sufficient to describe the system"ᄑ. In contrast to this standard scenario, the relevant state variables of a

\footnotetext{
${ }^{12} \mathrm{~A}$ molecular motor is a very complex enzyme with a huge number of degrees of freedom (see chapter 0 ). Within the present temperature ratchet model, the ATP hydrolyzation energy is thought to be quickly converted into a very irregular vibrational motion of these degrees of freedom, i.e. a locally increased apparent temperature. As this excess heat spreads out, the temperature decreases again. Thus, the internal degrees of freedom play a crucial role but are irrelevant in so far as they do not give rise to any additional slow, collective state variable.

${ }^{13}$ We also note that a current $\langle\dot{x}\rangle$ opposite to the force $F$ is not in contradiction with any kind of "stability criteria", cf. the discussion below 2.39).

${ }^{14}$ In passing we notice that the case $F=0$ in conjunction with a time-dependent temperature $T(t)$ is conceptually quite interesting: It describes a system which is at any given instant of time an equilibrium system in a non-equilibrium (typically far from equilibrium) state.

${ }^{15}$ Note that a fixed sequence of events does not necessarily imply a deterministic evolution in time.
} 
genuine Brownian motor are loosely coupled: Of course, some degree of interaction is indispensable for the functioning of the Brownian motor, but while $T(t)$ completes one temperature cycle, $x(t)$ may evolve in several essentially different ways (it is not "slaved" by $T(t))$.

The loose coupling between state variables is a salient point which makes the Brownian motor concept more than just a cute new look at certain very small and primitive, but otherwise quite conventional thermo-mechanical or even purely mechanical engines. In most cases of practical relevance, the presence of some amount of (not necessarily thermal) random fluctuations is therefore an indispensable ingredient of a genuine Brownian motor; exceptionally, deterministic chaos may be a substitue (cf. sections 5.4 and 5.8.2).

We remark that most of the specific ratchet models that we will consider later on do have a second relevant state variable besides ${ }^{\text {TO }} x(t)$. One prominent exception are the so-called Seebeck ratches, treated in section 6.1. In such a case the above condition of a loose coupling between state variables is clearly meaningless. This does, however, not imply that those are no genuine Brownian motors.

The important issue of whether the coupling between relevant state variables is loose or tight has been mostly discussed in the context of molecular motors [12, 16, 144] and has been given the suggestive name mechanochemical coupling, see also section 7.4.3 and 7.7. The general fact that such couplings of non-equilibrium enzymatic reactions to mechanical currents play a crucial role for numerous cellular transport processes is long known 23, 24.

\subsection{Curie's principle}

The main, and a priori quite counterintuitive observation from section 2.1 is the fact that no preferential direction of the random dynamics (2.5), (2.6) arises in spite of the broken spatial symmetry of the system. The next surprising observation from section 2.6 is the appearance of the ratchet effect, i.e. of a finite current $\langle\dot{x}\rangle$, for the slightly modified temperature ratchet model (2.6), (2.48) in spite of the absence of any macroscopic static forces, gradients (of temperature, concentration, chemical potentials etc.), or biased time-dependent perturbations. Here the word "macroscopic" refers to "coarse grained" effects that manifest themselves over many spatial periods $L$. Of course, on the "microscopic" scale, a static gradient-force $-V^{\prime}(x)$ is acting in (2.6), but that averages out to zero for displacements by multiples of $L$. Similarly, at most time-instants $t$, a non-vanishing thermal force $\xi(t)$ is acting in (2.6), but again that averages out to zero over long times or when an entire statistical ensemble is considered.

The first observation, i.e. the absence of a current at thermal equilibrium, is a consequence of the second law of thermodynamics. In the second above mentioned situation, giving rise to a ratchet effect, this law is no longer applicable, since the system is not in a thermal equilibrium state. So, in the absence of this and any other prohibitive a priori reason, and in view of the fact that, after all, the spatial symmetry

In particular, small ("microscopic") fluctuations which can be described by some environmental (equilibrium or not) noise are still admissible.

${ }^{16}$ While this second state variable obviously influences $x(t)$ in some or the other way, a corresponding back-reaction may or may not exists. The latter case is exemplified by the temperature ratchet model. 
of the system is broken, the manifestation of a preferential direction for the particle motion appears to be an almost unavoidable educated guess.

This common sense hypothesis, namely that if a certain phenomenon is not ruled out by symmetries then it will occur, is called Curie's principle凹 [147]. More precisely, the principle postulates the absence of accidental symmetries in the generic case. That is, an accidental symmetry may still occur as an exceptional coincidence or by finetuning of parameters, but typically it will not. Accidental symmetries are structurally unstable, an arbitrarily small perturbation destroys them [12], while a broken symmetry is a structurally stable situation.

In this context it may be worth noting that the absence of a ratchet effect at thermal equilibrium in spite of the spatial asymmetry is no contradiction to Curie's principle: The very condition for a system to be at thermal equilibrium can also be expressed in the form of a symmetry condition, namely the so-called detailed balance symmetry 98 101, 148,152 .

\subsection{Brillouin's paradox}

As mentioned in section 2.1.1, both Smoluchowski and Feynman have already pointed out the close similarity of the ratchet and pawl gadget from figure 2.1 with a Maxwell demon and also with the behavior of a mechanical valve. But also the analogy of such a ratchet device with an electrical rectifier, especially the asymmetric response to an external static force field (cf. figure 2.4), has been pointed out in Feynman's Lectures [2], see also Vol. III, section 14-4 therein. In this modified context of an electrical rectifier, the astonishing fact that random thermal fluctuations cannot be rectified into useful work is called Brillouin's paradox [3] and has been extensively discussed e.g. in 100, 153 157.

The main point of this discussion can be most easily understood by comparison with the corresponding tilted Smoluchowski-Feynman ratchet model (2.34). Furthermore, we focus on the case of an electrical circuit with a semiconductor diod $\mathrm{TH}$. With the entire circuit being kept at thermal equilibrium, at any finite temperature and conductance, a random electrical noise arises and it is prima facie indeed quite surprising that its rectification by the diode is impossible. The stepping stone becomes apparent in the corresponding Smoluchowski-Feynman ratchet model (2.34). While its response to an external force $F$ in equation (2.37) and figure 2.4 shares the typical asymmetric shape with a diode, it is now clearly wrong to phenomenologically describe the effect of the thermal noise in such a system by simply averaging the current $\langle\dot{x}\rangle$ from (2.37) with respect to $F$ according to the probability with which the thermal noise takes these values $F$. Rather, the correct modeling, which in particular consistently incorporates the common microscopic origin of friction and thermal noise, is represented by (2.34) (with $F=0$ ). In contrast, the response characteristics (2.37) is already the

\footnotetext{
${ }^{17}$ In the biophysical literature [23,24] the notion of Curie's principle (or Curie-Prigogine's principle) is mostly used for its implications in the special case of linear response theory (transport close to equilibrium) in isotropic systems, stating that a force can couple only to currents of the same tensorial order, see also 145, 146.

${ }^{18}$ To be precise, detailed balance is necessary but not sufficient for thermal equilibrium [101, 148]. Conversely, detailed balance is sufficient but not necessary for a vanishing particle current $\langle\dot{x}\rangle$.

${ }^{19} \mathrm{~A}$ tube diode requires permanent heating and it is not obvious how to reconcile this with the condition of thermal equilibrium.
} 
result of an averaging over the thermal noise under the additional assumption that $F$ is practically constant on the typical transient time scales of the emerging current $\langle\dot{x}\rangle$. It is clear, that we do not recover the full-fledged noisy dynamics (2.34) by replacing phenomenologically $F$ by $\xi(t)$ in (2.37), notwithstanding the fact that in (2.34) these two quantities indeed appear in the same way. The close analogy of this situation with that in a semiconductor diode becomes apparent by considering that also in the latter case the asymmetric response characteristics is the result of a thermal diffusion process of the electrons near the interface of the n-p junction under quasi-static conditions and after averaging out the thermal fluctuations.

This example (see also [158] for another such example) demonstrates that the correct modeling of the thermal environment is not always obvious. Especially, taking the averaged macroscopic behavior of the system as a starting point for a phenomenological modeling of the noisy dynamics may be dangerous outside the linear response regime, as van Kampen and others are emphasizing since many years [100]. Much safer is a microscopic staring point in order to consistently capture the common origin of the dissipation and the fluctuations in the actual system of interest, as exemplified in sections 2.1.2, 3.4.1, and 8.1.

Away from thermal equilibrium, the realization of the ratchet effect by diodes and other semiconductor heterostructures is further discussed in sections 6.1 and 8.4 .

\subsection{Asymptotic analysis}

In the remainder of this chapter, we continue our exploration of the temperature ratchet model (2.34), (2.47), (2.48) with the objective to understand in somewhat more detail the behavior of the particle current $\langle\dot{x}\rangle$ at zero load $F=0$ as a function of various parameters of the model. Since a closed analytical solution of the Fokker-Planck equation (2.52) is not possible in general, we have to recourse to asymptotic expansions and qualitative physical arguments, complemented by accurate numerical results for a few typical cases. In the present, somewhat more techical section we analyse the behavior of the particle current for asymptotically slow and fast temperature oscillations.

For asymptotically slow temporal oscillations in (2.48) the time- and ensembleaveraged particle current $\langle\dot{x}\rangle$ approaches zero $\mathbb{D}$. Considering that $\mathcal{T} \rightarrow \infty$ means a constant $T(t)$ during any given, finite time interval, this conclusion $\langle\dot{x}\rangle \rightarrow 0$ is physically quite obvious. It can also be be formally confirmed by the observation that

$$
\hat{P}^{\mathrm{ad}}(x, t):=Z(t)^{-1} e^{-V(x) / k_{B} T(t)}
$$

with $Z(t):=\int_{0}^{L} d x e^{-V(x) / k_{B} T(t)}$ solves the Fokker-Planck equation (2.52) in arbitrarily good approximation for sufficiently large $\mathcal{T}$ and $F=0$. Comparison with (2.31) shows that this so-called adiabatic approximation (2.55) represents an accompanying or instantaneous equilibrium solution in which the time $t$ merely plays the role of a parameter. Introducing (2.55) into (2.53) with $F=0$ indeed confirms the expected result $\langle\dot{x}\rangle=0$.

Turning to finite but still large $\mathcal{T}$, one expects that $\langle\dot{x}\rangle$ decreases proportional to $\mathcal{T}^{-1}$ in the general case. In the special case that $T(t)$ is symmetric under time

\footnotetext{
${ }^{20}$ In the following we tacitly restrict ourselves to smooth $T(t)$, like e.g. in (2.51). For discontinuous $T(t)$, for instance (2.50), the conclusion $\langle\dot{x}\rangle \rightarrow 0$ for $\mathcal{T} \rightarrow \infty$ remains valid, but the reasoning has to be modified.
} 
inversion 21 , as for instance in (2.50), (2.51), the current $\langle\dot{x}\rangle$ must be an even function of $\mathcal{T}$ and thus typically decreases proportional to $\mathcal{T}^{-2}$ for large $\mathcal{T}$. Furthermore, our considerations along the lines of figure 2.6 suggest that, at least for potentials with only one maximum and minimum per period $L$, the current $\langle\dot{x}\rangle$ approaches zero from above if the minimum is closer to the adjacent maximum to the right, and from below otherwise. Here and in the following, we tacitly assume that apart form the variation of the time-period $\mathcal{T}$ itself, the shape of $T(t)$ does not change, i.e.

$$
\hat{T}(h):=T(\mathcal{T} h)
$$

is a $\mathcal{T}$-independent function of its dimensionless argument $h$ with period 1 .

Addressing small $\mathcal{T}$, i.e. fast temperature oscillations, it is physically plausible that the system cannot follow any more these oscillations and thus behaves for $\mathcal{T} \rightarrow 0$ like in the presence of a constant averaged temperature

$$
\bar{T}:=\frac{1}{\mathcal{T}} \int_{0}^{\mathcal{T}} d t T(t)=\int_{0}^{1} d h \hat{T}(h)
$$

Within this so-called sudden approximation we thus recover an effective SmoluchowskiFeynman ratchet dynamics (2.6). In other words, we expect that $\langle\dot{x}\rangle \rightarrow 0$ for $\mathcal{T} \rightarrow 0$. This behavior is confirmed by the analytical perturbation calculation in Appendix C, which yields moreover the leading order small- $\mathcal{T}$ result [118]

$$
\begin{aligned}
& \langle\dot{x}\rangle=\mathcal{T}^{2} B \int_{0}^{L} d x V^{\prime}(x)\left[V^{\prime \prime}(x)\right]^{2}+\mathcal{O}\left(\mathcal{T}^{3}\right) \\
& B:=\frac{4 L \int_{0}^{1} d h\left[\int_{0}^{h} d \hat{h}(1-\hat{T}(\hat{h}) / \bar{T})\right]^{2}}{\eta^{3} \int_{0}^{L} d x e^{V(x) / k_{B}} \bar{T} \int_{0}^{L} d x e^{-V(x) / k_{B} \bar{T}}} .
\end{aligned}
$$

Note that $B$ is a strictly positive functional of $T(t)$ and $V(x)$ and is independent of $\mathcal{T}$.

The most remarkable feature of (2.58) is that there is no contribution proportional to $\mathcal{T}$ independently of whether $T(t)$ is symmetric under time inversion or not. More according to our expectation is the fact that the current vanishes for very weak thermal noise, as a closer inspection of (2.58) implies: Similarly as for the weak noise analysis in section 2.5.1, for $\bar{T} \rightarrow 0$ the particles can never leave the local minima of the potential $V(x)$. In the opposite limit, i.e. for $\bar{T} \rightarrow \infty$, the potential should play no role any more and one expects again that $\langle\dot{x}\rangle \rightarrow 0$, cf. section 3.7. A more careful perturbative analysis of the high-temperature limit confirms this expectation. On the other hand, equation (2.58) predicts a finite limit for $\bar{T} \rightarrow \infty$, implying that the limits $\bar{T} \rightarrow \infty$ and $\mathcal{T} \rightarrow 0$ cannot be interchanged in this perturbative result. In other words, the correction of order $\mathcal{O}\left(\mathcal{T}^{3}\right)$ in (2.58) approaches zero for any finite $\bar{T}$ as $\mathcal{T} \rightarrow 0$, but is no longer negligible if we keep $\mathcal{T}$ fixed (however small) and let $\bar{T} \rightarrow \infty$.

The above predictions are compared with accurate numerical solutions in figure 2.7 for a representative case, showing very good agreement.

\footnotetext{
${ }^{21}$ Time inversion symmetry means that there is a $\Delta t$ such that $T(-t)=T(t+\Delta t)$ for all $t$.
} 


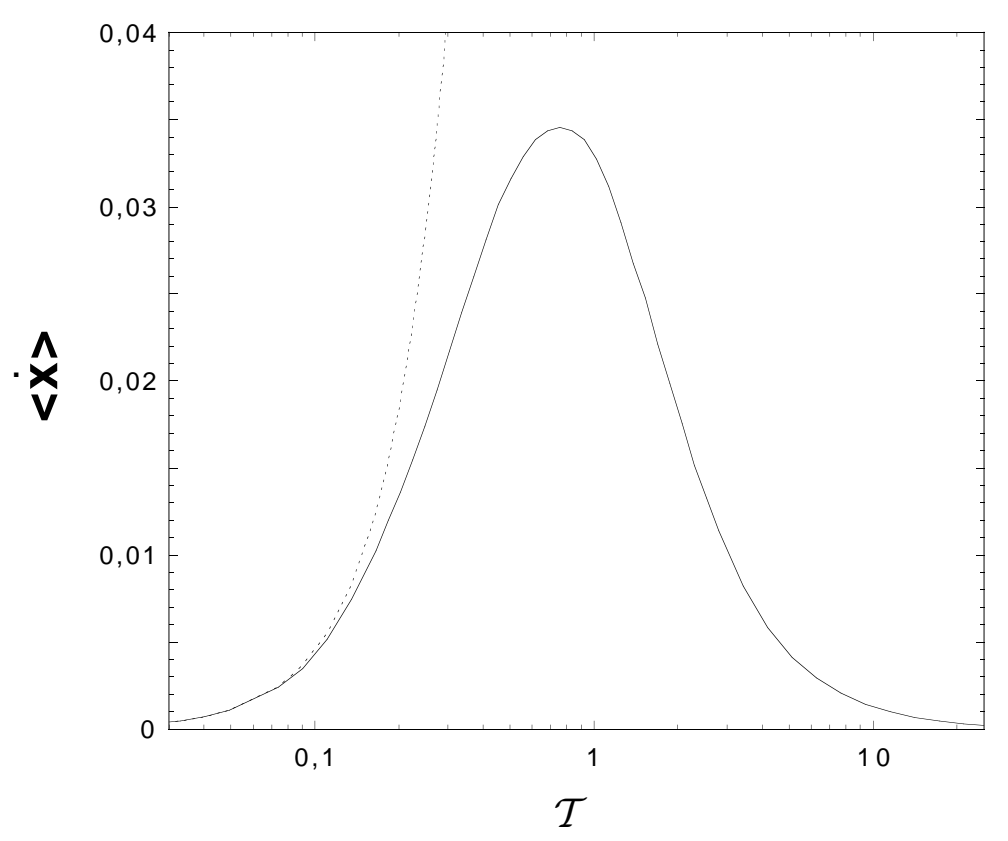

Figure 2.7: Average particle current $\langle\dot{x}\rangle$ versus period $\mathcal{T}$ for the temperature ratchet dynamics (2.3), (2.34), 2.47), 2.51) in dimensionless units (see section A.4 in Appendix A). Parameter values are $F=0, \eta=L=k_{B}=1, V_{0}=1 / 2 \pi, \vec{T}=0.1$, $A=0.7$. Solid: Time- and ensemble averaged current (2.53) by numerically evolving the Fokker-Planck equation (2.52) until transients have died out. Dotted: Analytical small- $\mathcal{T}$ asymptotics from (2.58).

\subsection{Current inversions}

The most basic qualitative prediction, namely that generically $\langle\dot{x}\rangle \neq 0$, is a consequence of Curie's principle. In this section we show that under more general conditions than in section 2.6.3, even the sign of the current $\langle\dot{x}\rangle$ may be already very difficult to understand on simple intuitive grounds, not to speak of its quantitative value. This leads us to another basic phenomenon in Brownian motor systems, namely the inversion of the current direction upon variation of a system parameter. Early observations of this effect have been reported in [35, 37, 39, 42, 159, 160]; here we illutrate it once more for our stantard example of the the temperature ratchet.

Since the quantity $B$ from (2.59) is positive, it is the sign of the integral in (2.58) which determines the direction of the current. For the specific ratchet potential (see equation (2.3) and figure 2.2) used in figure 2.7 this sign is positive, but one can easily find other potentials $V(x)$ for which this sign is negative. By continuously deforming one potential into the other one can infer that there must exist an intermediate $V(x)$ with the property that the particle current $\langle\dot{x}\rangle$ is zero at some finite $\mathcal{T}$-value. In the generic case, the $\langle\dot{x}\rangle$-curve passes with a finite slope through this zero-point, implying 118] the existence of a so-called "current inversion" of $\langle\dot{x}\rangle$ as a function of $\mathcal{T}$. An example of a potential $V(x)$ exhibiting such a current inversion is plotted in figure 2.8 and the resulting current in figure 2.9. As compared to the example from figure 2.2, the modification of the ratchet potential in figure 2.8 looks rather harmless. Especially, the explanation of a positive current $\langle\dot{x}\rangle>0$ for large $\mathcal{T}$ according to figure 2.5 
still applies. However, for small-to-moderate $\mathcal{T}$ this modification of the potential has dramatic consequences for the current in figure 2.9 as compared to figure 2.7.

Once a current inversion upon variation of one parameter of the model $(\mathcal{T}$ in our case) has been established, an inversion upon variation of any other parameter (for instance the friction coefficient $\eta$ ) can be inferred along the following line of reasoning [161]: Consider a current inversion upon variation of $\mathcal{T}$, say at $\mathcal{T}_{0}$, as given, while $\eta$ is kept fixed, say at $\eta_{0}$. Let us next consider $\mathcal{T}$ as fixed to $\mathcal{T}_{0}$ and instead vary $\eta$ about $\eta_{0}$. In the generic case the current $\langle\dot{x}\rangle$ as a function of $\eta$ will then go through its zero-point at $\eta_{0}$ with a finite slope, meaning that we have obtained the proposed current inversion upon variation of $\eta$, see figure 2.10.

In other words, Brownian particles with different sizes will have different friction coefficients $\eta$ and will thus move in opposite directions when exposed to the same thermal environment and the same ratchet potential. Had we not neglected the inertia effects $m \ddot{x}(t)$ in (2.1), such a particle separation mechanism also with respect to the mass $m$ could be inferred along the above line of reasoning, and similarly for other dynamically relevant particle properties!

Promising applications of such current inversion effects for particle separation methods, based on the ratchet effect, are obvious. Another interesting aspect of current inversions arises from the observation that structurally very similar molecular motors may travel in opposite directions on the same intracellular filament (see chapter 7). If we accept the temperature ratchet as a crude qualitative model in this context (cf. section 2.6.3), it is amusing to note that also this feature can be qualitatively reproduced: If two types of molecular motors are known to differ in their ATP consumption rate $1 / \mathcal{T}$, or in their friction coefficient $\eta$, or in any other parameter appearing in our temperature ratchet model, then it is possible to figure out a ratchet potential $V(x)$ such that they move indeed in opposite directions.

For a more general discussion of current inversion effects we refer to section 3.6 below. Additional material on the temperature ratchet model is contained in section 6.3 .

\footnotetext{
${ }^{22}$ See also section $\sqrt{3.6}$ for a detailed proof.
} 


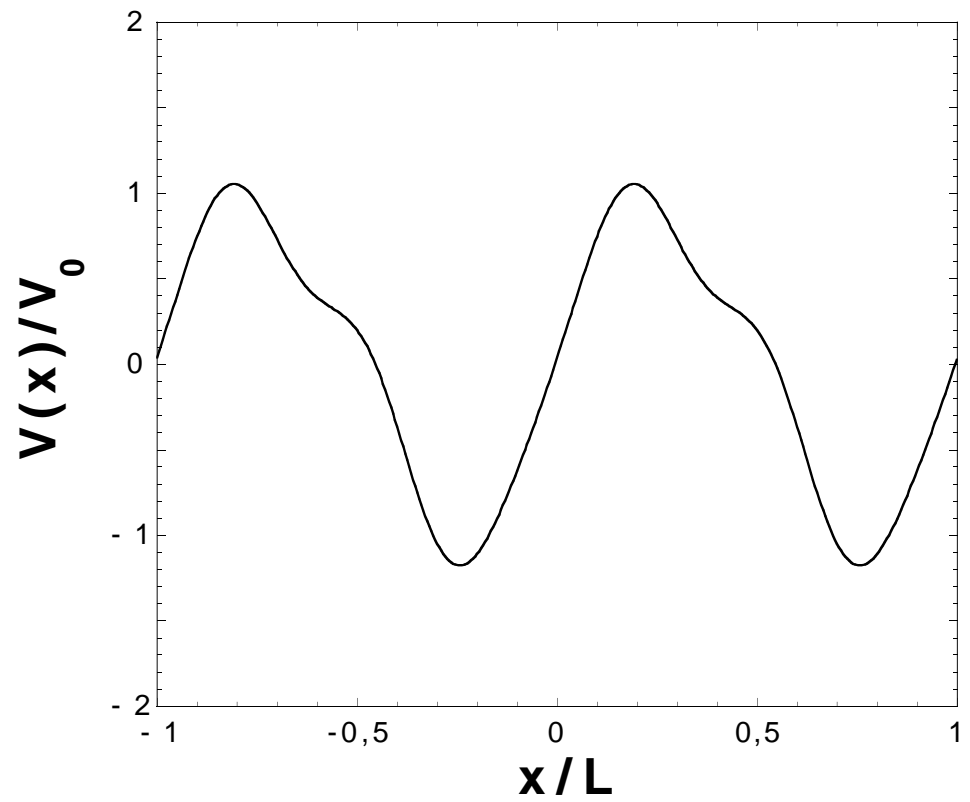

Figure 2.8: The ratchet potential $V(x)=V_{0}[\sin (2 \pi x / L)+0.2 \sin (4 \pi(x / L-0.45))+$ $0.1 \sin (6 \pi(x / L-0.45))]$.

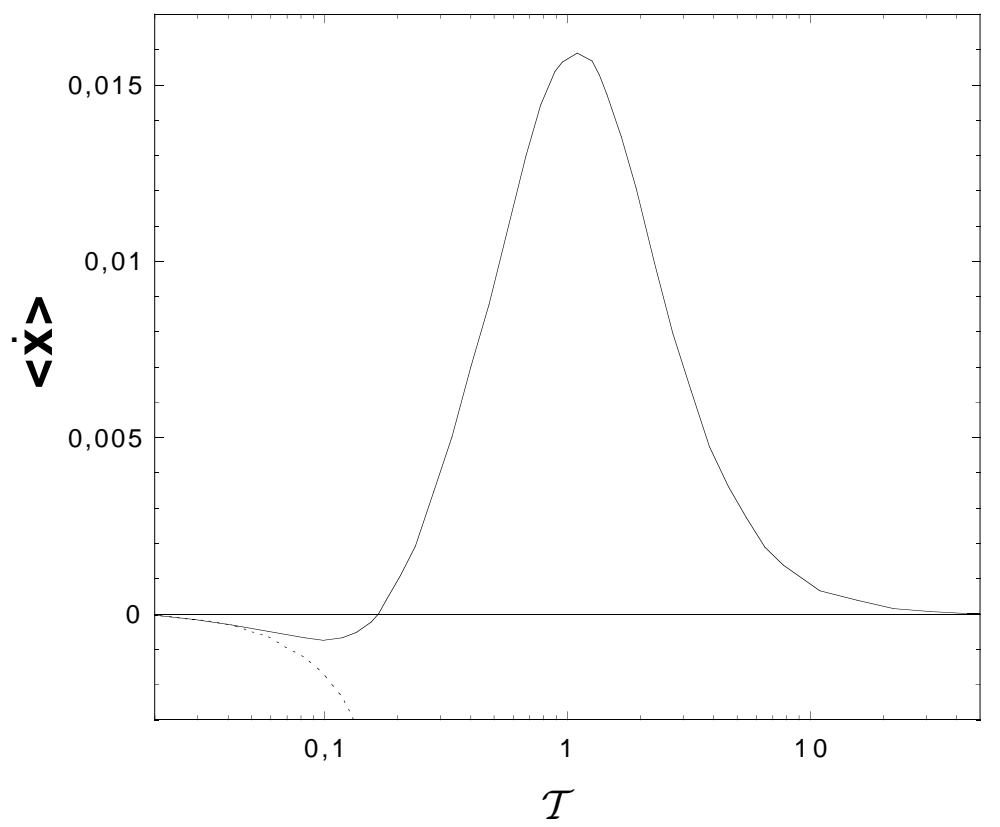

Figure 2.9: Same as figure 2.7 but for the ratchet potential from figure 2.8. 


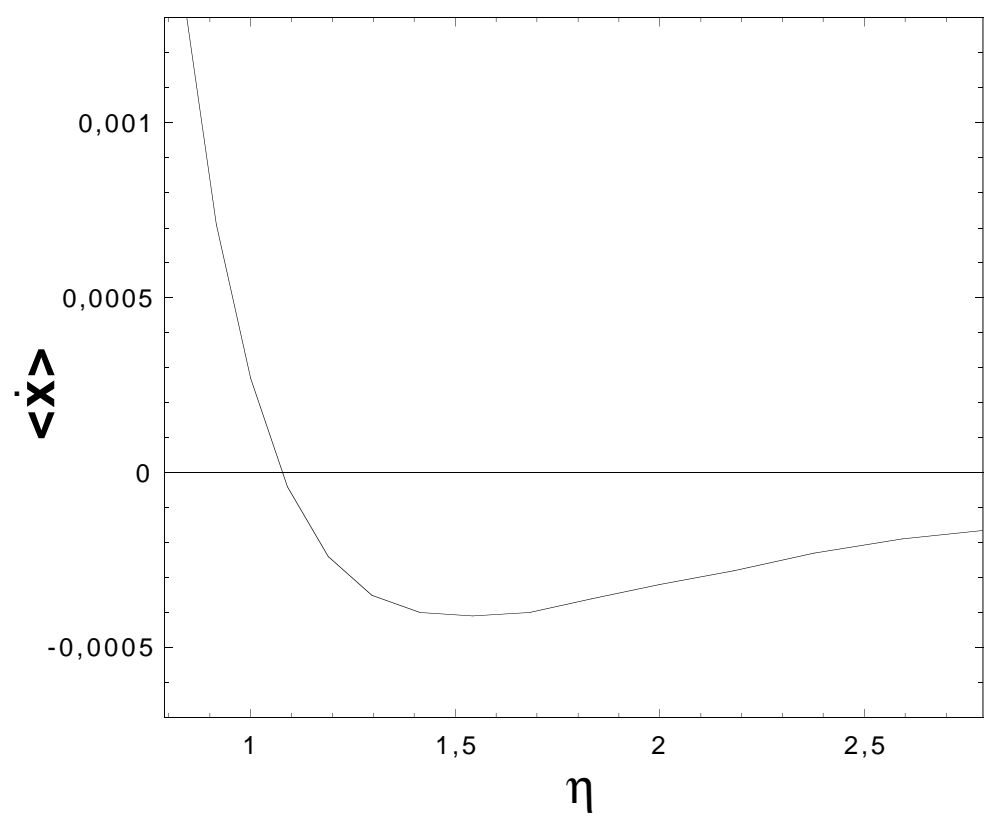

Figure 2.10: Same as in figure 2.9 but with a fixed period $\mathcal{T}=0.17$ (i.e. close to the inversion point in figure 2.9) and instead with a varying friction coefficient $\eta$. 


\section{Chapter 3}

\section{General Framework}

In chapters 349 we will review theoretical extensions and their experimental realizations of the concepts which were introduced by means of particularly simple examples in chapter 2. In the present chapter, we provide a first overview and general framework for the more detailed discussion in the subsequent chapters: The main classes of ratchet models and their physical origin are introduced. Symmetry considerations regarding the occurence or not of a finite particle current (ratchet effect) are a second important issue, complemeted by a general method of tailoring current inversions. Finally, a general treatment is provided for the asymptotic regimes of weak and strong noise-strength and of weak non-equilibrium perturbations. Specific examples and applications of these general concepts are mostely postponed to later chapters

\subsection{Working model}

In hindsight, the essential ingredient of the ratchet effect from section 2.6 .2 was a modification of the Smoluchowski-Feynman ratchet model (2.6) so as to permanently keep the system away from thermal equilibrium. We have exemplified this procedure by a periodic variation of the temperature (2.48) but there clearly exists a great variety of other options. In view of this example, the following guiding principles should be observed also in more general cases: (i) We require spatial periodicity and either invariance or periodicity under translations in time. (ii) All forces and gradients have to vanish after averaging over space ("coarse graining" over many spatial periods), over time (in the case of temporal periodicity), and over statistical ensembles (in the case of random fluctuations). (iii) The system has to be driven permanently out of thermal equilibrium and there should be no symmetries which prohibit a ratchet effect a priori. According to Curie's principle we can therefore expect the generic appearance of a finite particle current $\langle\dot{x}\rangle$. (iv) In view of the title of our present study, we will mostly (not exclusively) focus on models with a finite amount of thermal noise

According to these preliminary considerations, we adopt as our basic working model the overdamped one-dimensional stochastic dynamics

$$
\begin{aligned}
& \eta \dot{x}(t)=-V^{\prime}(x(t), f(t))+y(t)+F+\xi(t) \\
& \langle\xi(t) \xi(s)\rangle=2 \eta k_{B} T \delta(t-s),
\end{aligned}
$$

\footnotetext{
${ }^{1}$ Note that (iv) is not a consequence of (iii), as demonstrated by any dissipative driven system at zero temperature.
} 
where $\eta$ is the viscous friction coefficient and $V^{\prime}(x, f):=\partial V(x, f) / \partial x$. With respect to its spatial argument $x$, the potential is periodic for all possible arguments $f(t)$, i.e.

$$
V(x+L, f(t))=V(x, f(t))
$$

for all $t$ and $x$. Along the same line of reasoning as in section A.4 of Appendix A, inertia effects are neglected and thermal fluctuations are modeled by uncorrelated (white) Gaussian noise $\xi(t)$ of zero average and intensity $2 \eta k_{B} T$ (see also section 3.4.1 below). Finally, $F$ is a constant "load" force. Since such a bias violates the above requirement (ii), it should not be considered as part of the system but rather as an externally imposed perturbation in order to study its response behavior.

We furthermore assume that $f(t)$ and $y(t)$ are either periodic or stochastic functions of time $t$. In the case that one or both of them are a stochastic process, we make the simplifying assumption that this process is stationary, and in particular statistically independent of the thermal noise $\xi(t)$ and of the state of system $x(t)$. With the symbol $\langle\cdot\rangle$ we henceforth indicate an ensemble average over realizations of the stochastic dynamics (3.1), i.e. a statistical average with respect to the thermal noise $\xi(t)$ and in addition with respect to $f(t)$ and/or $y(t)$ if either of them is a stochastic process.

The quantity of central interest is the average particle current (cf. (2.15))

$$
\langle\dot{x}\rangle:=\langle\dot{x}(t)\rangle
$$

In most cases ${ }^{2}$ we will furthermore focus on the behavior of the particle current in the long-time limit $t \rightarrow \infty$ (cf. section 2.4). If both $f(t)$ and $y(t)$ are random processes in time, then the existence of a stationary long-time limit and its uniqueness are taken for granted. If $f(t)$ and/or $y(t)$ is a periodic function of $t$, then the existence of a unique periodic long-time behavior is assumed and a time average is tacitly incorporated into $\langle\dot{x}\rangle$ (cf. equation (2.53)). Both, for random and periodic processes, this long time limit of the current can usually be identified, due to ergodicity reasons, with the time averaged velocity of a single realization $x(t)$ of the stochastic dynamics (3.1), i.e. with probability 1 we have that

$$
\langle\dot{x}\rangle=\lim _{t \rightarrow \infty} \frac{x(t)}{t}
$$

independent of the initial condition $x(0)$.

A further quantity of interest is the effective diffusion coefficient

$$
D_{\text {eff }}:=\lim _{t \rightarrow \infty} \frac{1}{2 t}\left\langle[x(t)-\langle x(t)\rangle]^{2}\right\rangle
$$

For $-V^{\prime}(x(t), f(t))+y(t) \equiv 0$, the effective diffusion coefficient (3.6) agrees with the bare coefficient (2.11), independent of $F$. In general, its determination is a difficult

\footnotetext{
${ }^{2}$ There are only very few investigations on transient features of ratchet systems 162167 .

${ }^{3}$ Proof: The time averaged current from (3.4) can be rewritten as $\langle\dot{x}\rangle=\left\langle\lim _{t \rightarrow \infty} t^{-1} \int_{0}^{t} \dot{x}\left(t^{\prime}\right) d t^{\prime}\right\rangle=$ $\lim _{t \rightarrow \infty}\langle x(t)-x(0)\rangle / t$. The random process $x(t)-x(0)$ exhibits on top of the systematic drift $\langle x(t)-$ $x(0)\rangle / t$ a certain random dispersion (or diffusion) of the order $\sqrt{2 D_{\text {eff }} t}$ for large $t$, cf. equation (3.7). Due to the division by $t$ it follows that this dispersion is negligible, i.e. $\langle\dot{x}\rangle=\lim _{t \rightarrow \infty}[x(t)-x(0)] / t=$ $\lim _{t \rightarrow \infty} x(t) / t$ with probability 1 for any realization $x(t)$.
} 
time-dependent problems 1 . and we will restrict ourselves to a few special cases.

On a sufficiently coarse grained scale in space, the motion of the particle $x(t)$ takes the form of single "hopping" events which are independent of each other and equally distributed. According to the central limit theorem [100], a statistical ensemble of particles $x(t)$ with initial condition $x(0)=x_{0}$ thus approaches a Gaussian distribution $117,168,174$

$$
P(x, t) \simeq \frac{1}{\sqrt{4 \pi D_{\text {eff }}}} \exp \left\{-\frac{\left[\langle\dot{x}\rangle t-x_{0}\right]^{2}}{4 D_{\text {eff }} t}\right\}
$$

for large times $t$. As far as the objective of particle separation is concerned, we see that not only a large difference or even opposite sign of the velocities $\langle\dot{x}\rangle$ is important (cf. section 3.6), but also the effective diffusion coefficients and the time $t$ (or, equivalently, the length $\langle\dot{x}\rangle t$ of the experimental device) play a crucial role [34, 170, 172, 174, 175], see also section 6.6. A purely diffusive $(\langle\dot{x}\rangle=0)$ particle separation scheme will be discussed in section 5.7.

Once in a while, certain extensions of the above framework will appear, e.g. an additional finite inertia term $m \ddot{x}(t)$ on the left hand side of (3.1) or two instead of one spatial dimensions, see e.g. in sections 5.4 and 5.5, respectively. Furthermore, models with a time or space dependent temperature in (3.2) will be discussed in sections 6.1 6.3, and similarly in section 6.4 models with a time or space dependent friction. Deviations of the spatial periodicity (3.3) may arise in the form of some amount of quenched spatial disorder (section 6.8) or as a superposition of several periodic contributions with incommensurate periods (section 4.5.1). The case of a spatially discretized state variable is reviewed in section 6.7. A class of models with a non-trivial dependence of the process $f(t)$ upon the state $x(t)$ of the system appears in section 6.2 and in chapter 7. Generalizations of a more drastic nature are addressed in chapters 8 and 9 .

If $y(t)$ is a periodic function of time, say

$$
y(t+\mathcal{T})=y(t)
$$

then we can assume without loss of generality that

$$
\int_{0}^{\mathcal{T}} d t y(t)=0
$$

thanks to the free constant $F$ in (3.1). Similarly, if $y(t)$ is a stationary stochastic process then we can assume that

$$
\langle y(t)\rangle=0 \text {. }
$$

Without loss of generality, it is also sufficient to concentrate on $f(t)$ which are unbiased in the same sense as in (3.9), (3.10).

As far as unbiased stationary random processes are concerned, two examples are of particular importance due to their archetypal simplicity. To be specific, we will use the

\footnotetext{
${ }^{4}$ While for the current it is sufficient to consider an auxiliary dynamics with periodic boundary conditions, which approaches a stationary (if $f(t)$ and $y(t)$ are random processes) or periodic long time limit (cf. section 2.4), no such simplification is possible with respect to the effective diffusion coefficient. In particular, the effective diffusion coefficient is in general no longer related to the mobility via a generalized Einstein relation (2.11), i.e. $D_{\text {eff }}=k_{B} T \partial\langle\dot{x}\rangle / \partial F$ only holds when $f(t) \equiv 0, y(t) \equiv 0$, and $F=0$.
} 
symbol $f(t)$, while completely analogous considerations can of course be immediately transcribed to $y(t)$ as well. The first example is a so-called symmetric dichotomous noise or telegraphic noise [63, 176 178], i.e. a stochastic process which switches back and forth between two possible "states" $+\sigma$ and $-\sigma$ with a constant probability $\gamma$ per time unit. In the stationary state the distribution of the noise

$$
\rho(f):=\langle\delta(f-f(t))\rangle
$$

is thus given by

$$
\rho(f)=\frac{1}{2}[\delta(f-\sigma)+\delta(f+\sigma)]
$$

independent of the time $t$ in (3.11). One furthermore finds that the correlation is given by

$$
\langle f(t) f(s)\rangle=\sigma^{2} e^{-|t-s| / \tau},
$$

where $\tau:=1 / 2 \gamma$ is the correlation time and

$$
\sigma^{2}:=\left\langle f^{2}(t)\right\rangle=\int_{-\infty}^{\infty} d f f^{2} \rho(f)
$$

is the variance (independent of $t$ ).

Being abundant in natural systems as well as in technological applications, a stationary Gaussian distributed noise $f(t)$ is clearly a second type of random fluctuations that warrants to be analyzed in more detail. In the simplest case, these stationary Gaussian fluctuations are furthermore unbiased, and Markovian!. According to Doob's theorem [100], $f(t)$ is thus a so-called Ornstein-Uhlenbeck process [99, 101], characterized by a stationary probability distribution

$$
\rho(f)=\left(2 \pi \sigma^{2}\right)^{-1 / 2} e^{-f^{2} / 2 \sigma^{2}}
$$

and the same correlation as in (3.13). So, the variance $\sigma^{2}$ and the correlation time $\tau$ are the model parameters for both, dichotomous noise and Ornstein-Uhlenbeck noise.

\subsection{Symmetry}

\subsubsection{Definitions}

The potential $V(x, f(t))$ is called spatially symmetric or simply symmetric if there exists a $\Delta x$ such that

$$
V(-x, f(t))=V(x+\Delta x, f(t))
$$

for all $x$ and $t$. In other words, we will use the notions "symmetry" and "asymmetry" as synonyms for "spatial isotropy" and "anisotropy", respectively.

A further important symmetry regards the unbiased tilting process $y(t)$ : If $y(t)$ is periodic in time and there exists a $\Delta t$ such that $-y(t)=y(t+\Delta t)$ for all $t$ then we

\footnotetext{
${ }^{5}$ Note that $\sigma$ in $(3.14)$ is consistent with 3.12 ) and $(3.15)$.

${ }^{6}$ The future of $f(t)$ only depends on its present state, not on its past 101.
} 
call $y(t)$ "inversion symmetric" or simply symmetric. By performing the symmetry transformation twice, it follows that $y(t)=y(t+2 \Delta t)$ and under the assumption that $\mathcal{T}$ is the fundamental time-period, i.e. the smallest $\tau>0$ such that $y(t+\tau)=y(t)$, the symmetry condition takes the form [39]

$$
-y(t)=y(t+\mathcal{T} / 2) \text {. }
$$

If $y(t)$ is a stationary stochastic process, then we call it symmetric if all statistical properties of the process $-y(t)$ are the same as those of $y(t)$, symbolically indicated as

$$
-y(t) \hat{=} y(t)
$$

Examples are the symmetric dichotomous noise and the Ornstein-Uhlenbeck process as introduced at the end of the preceding subsection, or the symmetric Poissonian shot noise from section 5.1. Note that the assumption of an unbiased $y(t)$, see (3.9), (3.10), does not yet imply that $y(t)$ is symmetric.

Regarding nomenclature, an asymmetric potential is also called a ratchet potential. On the other hand, the dynamics (3.1) will be termed Brownian motor, ratchet dynamics, or simply ratchet not only if the potential $V(-x, f(t))$ is asymmetric but also if the driving $y(t)$ is asymmetric, while the potential may then be symmetric.

\subsubsection{Conclusions}

From the definition (3.16) it follows that a $L$-periodic potential $V(x, f(t))$ is symmetric if and only if it is of the general form

$$
V(x, f(t))=\sum_{n=1}^{\infty} a_{n}(f(t)) \cos (2 \pi n x / L) .
$$

Here and in the following, trivial freedoms in the choice of the $x$ - and $V$-origins are neglected. In the specific case (3.16) this means that we have silently set $\Delta x=0$ and $a_{0}(f(t))=0$ in (3.19). Similarly, one sees that the symmetry condition (3.17) for a periodic, deterministic driving $y(t)$ is equivalent to a Fourier representation of the general form

$$
y(t)=\sum_{n=1,3,5, \ldots} b_{n} \cos \left(\frac{2 \pi n t}{\mathcal{T}}+\phi_{n}\right)
$$

In the case that $y(t)$ is a stochastic process, the symmetry condition (3.18) is equivalent to the requirement that all its odd moments vanish [179, 180], i.e.

$$
\left\langle y\left(t_{1}\right) y\left(t_{2}\right) \cdots y\left(t_{2 n+1}\right)\right\rangle=0
$$

for all integers $n \geq 0$ and all times $7 t_{1}, t_{2}, \ldots, t_{2 n+1}$. Basically, the reason for this is that the stochastic process $y(t)$ is completely specified by the set of all its multiple-time joint probability distributions (Kolmogorov-theorem) and those are in turn completely fixed by all its moments [100]. On this basis, the equivalence of (3.18) and (3.21) follows.

\footnotetext{
${ }^{7}$ Here and in what follows we tacitly assume that all multiple-time moments of the precess $y(t)$ exists.
} 
If the potential $V(x, f(t))$ respects the symmetry condition (3.19) and the driving $y(t)$ either (3.20) or (3.21) then we can conclude that the long time averaged particle current (3.5) vanishes in the absence of a static tilt $F$ in (3.1), i.e.

$$
\langle\dot{x}\rangle=\lim _{t \rightarrow \infty} \frac{x(t)}{t}=0 .
$$

For a proof, we recall that the current in (3.5) is independent of the initial condition $x(0)$ and we may thus choose $x(0)=0$. If both, $V(x, f(t))$ and $y(t)$ are symmetric according to (3.16)-(3.18), or equivalently (3.19)-(3.21), then it follows that a realization $x(t)$ of the random process (3.1), (3.2) with $F=0$ in (3.1) occurs with the same probability as its mirror image $-x(t)$. Hence, we can infer from (3.5) that $\langle\dot{x}\rangle=-\langle\dot{x}\rangle$, implying (3.22). In other words, the main conclusion of this subsection is that if both, the potential $V(x, f(t))$ and the driving $y(t)$ are symmetric according to (3.10)-(3.21) then the average particle current (3.5) is zero.

If the potential and the driving $y(t)$ do not both satisfy their respective symmetry criteria, then, according to Curie's principle, a finite average current is expected in the generic case. The exceptional (non-generic) cases with zero current (3.22) in spite of a broken symmetry are either in some sense "accidental" [12 (analogous to the current inversion in figure 2.9) or can be traced back to certain "hidden" symmetry reasons of a more fundamental and systematic nature. Examples of the latter type will be the subject of sections 3.5 and 6.4.1, see also the concluding remarks in chapter 10 .

The generalization of these symmetry considerations to the case of a quasiperiodic driving $y(t)$ is due to [181, while an extension to two-dimensional systems (cf. section 5.5) and models with an internal degree of freedom (cf. section 6.5) is contained in 182, 183] and 184, respectively.

\subsection{Main ratchet types}

In this section we introduce the classification scheme underlying the organization of chapters 146. Some general physical considerations complementing this abstract classification are summarized in 3.4 .

As already discussed in section 2.6, of foremost interest is usually the current $\langle\dot{x}\rangle$ in the long time limit in the absence of a static tilt $F$ in (3.1). If both, the potential $V(x, f(t))$ and the tilting force $y(t)$ are symmetric, then a vanishing current will be the result (see preceding subsection). The following classification of the different types of ratchet models is on the one hand based on the systematic breaking of this symmetry, on the other hand it follows to some extent the historically grown, non-systematic nomenclature.

There are two fundamental classes of ratchet models arising from (3.1). The first one are models with $y(t) \equiv 0$, which we denote as pulsating ratchets. The second are models with $f(t) \equiv 0$, called tilting ratchets [49].

Within the realm of pulsating ratchets $(y(t) \equiv 0)$, the first main subclass is obtained when $f(t)$ in (3.1) is additive, i.e.

$$
V(x, f(t))=V(x)[1+f(t)] .
$$

Such models carry the name fluctuating potential ratchets. The summand 1 is a matter of convention, reflecting a kind of "unperturbed" contribution to the total potential. 
The class of fluctuating potential ratchets contains as special case the on-off ratchets when $f(t)$ can take only two possible values, one of them being -1 (potential "off"). Without loss of generality the other value can then be assumed to be +1 .

One readily sees that the potential $V(x, f(t))$ on the left hand side of $(3.23)$ satisfies the symmetry condition (3.16) if and only if $V(x)$ on the right hand side of (3.23) is symmetric as well. Furthermore, it is obvious that a symmetric $V(x)$ in 3.23 always results in a vanishing current $\langle\dot{x}\rangle$, whatever the properties of $f(t)$ are. We will therefore focus on the simplest non-trivial scenario, namely asymmetric potentials $V(x)$ in combination with symmetric $f(t)$. As the word "fluctuating potential" already suggests, we will mainly focus on random $f(t)$, though periodic $f(t)$ are in principle meant to be equally covered by this name.

A second subclass of pulsating ratchets, called traveling potential ratchets, have potentials of the form

$$
V(x, f(t))=V(x-f(t)) .
$$

The most natural choice, already suggested by the name "traveling potential", are $f(t)$ with a systematic long time drift $u:=\lim _{t \rightarrow \infty} f(t) / t$. As a consequence, $f(t)$ can only be a veritable periodic function or stationary stochastic process after subtraction of this systematic drift. We will call such a model a genuine traveling potential ratchet scheme. This slight extension of our general framework will be justified by our demonstration that such a model is exactly equivalent either to a tilting ratchet or to a so-called improper traveling potential ratchet, for which already the "original" $f(t)$ is a periodic function or a stationary stochastic process. Within a traveling potential ratchet scheme, the potential $V(x, f(t))$ on the left hand side of (3.24) never satisfies the symmetry criterion (3.16), independently of whether the potential $V(x)$ on the right hand side is symmetric or not. Both, the genuine and improper schemes are therefore interesting to study since a symmetric potential $V(x)$ is sufficient for current generation. Especially, the word "ratchet" does not necessarily refer to an asymmetric potential $V(x)$ in this context.

Next we turn to the tilting ratchet scheme, characterized by $f(t) \equiv 0$ and thus

$$
V(x, f(t))=V(x)
$$

in (3.1). When $V(x)$ is a ratchet potential, then we will restrict ourselves mostly to symmetric $y(t)$. If $y(t)$ is a stochastic process, we speak of a fluctuating force ratchet. The case of a tilting ratchet with a periodic driving $y(t)$ is of particular experimental relevance and carries the obvious name rocking ratchet [42].

Coming to symmetric potentials $V(x)$ in (3.25), a broken symmetry of $y(t)$ turns out to be necessary and generically also sufficient for a finite current $\langle\dot{x}\rangle$. We will use the name asymmetrically tilting ratchet if $y(t)$ is not symmetric, independently of whether it is a periodic function or a stochastic process, and independently of whether $V(x)$ is symmetric or not.

A further important class of ratchets is given by models of the form (3.1), (3.3) with both $f(t) \equiv 0$ and $y(t) \equiv 0$ but instead with a space or time dependent temperature $T$ in (3.2). They carry the names Seebeck ratchets and temperature ratchets, respectively. In the case of a space dependent temperature, $T(x)$ is assumed to have the same periodicity $L$ as the potential $V(x)$. In the case of a time-dependent temperature $T(t)$, again a periodic or stochastic, stationary behavior is assumed. We anticipate that 
models of this type are obviously not pulsating ratchets in the original sense, but as will be demonstrated in sections 6.1 and 6.3 - they can be mapped onto genuine pulsating ratchets. Also discussed in this context (section 6.2) will be so-called Feynman ratchets, i.e. the extension of the isothermal Smoluchowski-Feynman ratchet and pawl from figure 2.1 to the non-equilibrium case involving simultaneously two thermal baths at different temperatures. Starting with a faithful two-dimensional model, which is in fact equivalent to a generalized fluctuating potential scheme, additional simplifications give rise to a one-dimensional, Seebeck ratchet-like approximative description. Finally, the case of a varying friction coefficient in (2.11) (temporal and/or spatial) is denoted as friction ratchet. In section 6.4.1, we show that such a modification of the Smoluchowski-Feynman ratchet model (2.5), (2.6) does not break the detailed balance symmetry and thus does not admit a ratchet effect 9 , in contrast to a modified, so-called memory-friction modelling as discussed in section 6.4.3.

We remark that the main idea of the above classification scheme is the identification of different basic minimal models. Clearly, there are many possible combinations and generalizations, e.g. a simultaneously pulsating and tilting ratchet or the simultaneous breaking of more than one symmetry. Especially, there exist numerous pulsating ratchet schemes involving potentials $V(x, f(t))$ which go beyond the special cases of fluctuating potential and traveling potential ratchets. Such generalizations will not be systematically analyzed since no fundamentally new phenomena are expected. They are, however, realized in some interesting experimental systems and will be discussed in such specific contexts.

\subsection{Physical basis}

The physical situations in which a model of the form (3.1)-(3.3) may arise are extremely diverse. Therefore, a systematic discussion makes little sense and we restrict ourselves in this section to a few general remarks before turning to the various concrete systems in the subsequent chapters.

The stochastic process $x(t)$ in (3.1) has as state space the entire real axis and for simplicity is often called a "Brownian particle". While in some cases, $x(t)$ indeed represents the position of a true physical particle, in others it may also refer to some quite different type of collective degree of freedom or relevant (slow) state variable. Examples which we will encounter later on are the chemical reaction coordinate of an enzyme, the geometrical configuration or some other internal degree of freedom of a molecule, the position of the circular ratchet in figure 2.1 with respect to the pawl, the Josephson phase in a SQUID (superconducting quantum interference device), and the collective angular variable in phenomenological models for pinned charge density waves. In many cases the state variable $x(t)$ is thus originally of a phase-like nature with a circle as state space. The expansion to the real axis is immediate and has the additional advantage of counting the number of revolutions. Accordingly, the periodicity (3.3) - a central property of our model - may have its root either in a true spatial periodicity of the physical system or in the phase-like nature of the original state variable.

\footnotetext{
${ }^{8}$ Especially, such a modification requires a correct handling of the non-trivial overdamped limit $m \rightarrow 0$ in 2.1 , see section 6.4.1.
} 


\subsubsection{Thermal environment}

Another central feature in our working model (3.1), (3.2) is the presence of a thermal environment. In this section we continue and extend our discussion from section 2.1.2 (see also section A.1 in Appendix A) regarding the physical origin of the particularly simple form of the system-bath interaction in (3.1), (3.2), namely an additive white Gaussian noise and an additive viscous dissipation proportional to the instantaneous system velocity.

Adopting a phenomenological approach, in many cases [66, 99, 101] such an ansatz has proven to provide a rather faithful modeling, justified by its agreement with experimental measurements and the intuitive physical picture that has emerged on the basis of those observations.

A different approach starts with a microscopic modeling of the system of actual interest and its thermal environment. In the following we briefly sketch the main steps of such an approach. For a somewhat more detailed illustration of these general concepts for specific physical examples we also refer to sections 7.2, 7.3, and 8.1. On the one hand, such a microscopic foundation provides a physical picture of why the phenomenological modeling (3.1), (3.2) is successful in such a wide variety of different systems. On the other hand, a feeling for the conditions under which such a modeling is valid is acquired as well as an idea of how to modify the model when they break down.

Our starting point is a Hamiltonian of the general form

$$
H=\frac{p^{2}}{2 m}+V_{s}(x)+\sum_{j=1}^{N} \frac{p_{j}^{2}}{2 m_{j}}+V_{b}\left(x, x_{1}, \ldots, x_{N}\right),
$$

where $x$ and $p$ are the coordinate and momentum of the actual system of interest, while $x_{j}$ and $p_{j}$ are those of the numerous $(N \gg 1)$ microscopic degrees of freedom of the environment. The last term in (3.26) is a general interaction potential, including the coupling between system and environment. To keep things simple, we restrict ourselves to a single relevant (i.e. "slow") state variable $x(t)$, e.g. the cartesian coordinate of a particle in the absence of magnetic fields or the Josephson phase in a SQUID. We remark that in other cases, e.g. the chemical reaction coordinate of an enzyme, the geometrical configuration, or some other internal degree of freedom of a molecule, the respective "slow" relevant state variable $x(t)$ is usually a generalized coordinate (a non-trivial function of the cartesian coordinates of the nuclei, cf. section 7.2), and similarly for the "fast" bath degrees of freedom $x_{j}(t)$. As a consequence, the kinetic energy terms are of a more complicated form than in (3.26) and with respect to the potential terms there exists no longer a meaningful distinction between the "actual system of interest" and the "environment plus the system-bath-coupling". In those cases, our general line of reasoning remains still valid, but the detailed calculations become more involved [92, 93, 150, 185].

\section{Elimination of the bath degrees of freedom}

Having set the stage (3.26), our next goal is to get rid of the environmental degrees of freedom $x_{j}(t)$. To this end, we start by formally solving the respective equations of motions for any prescribed function $x(t)$ and initial conditions $\phi_{0}:=$ 
$\left(x_{1}(0), p_{1}(0), \ldots, x_{N}(0), p_{N}(0)\right)$ at time $t_{0}=0$. In other words, we can write down (formal) solutions $x_{j}\left(t,\left[x\left(t^{\prime}\right)\right], \phi_{0}\right)$ which are at the same time functions of $t$ and $\phi_{0}$ and functionals of the (explicitly still unknown) system dynamics $x\left(t^{\prime}\right)$ for $t^{\prime} \in[0, t]$. Introducing these solutions into the equation of motion for the system $x(t)$ is equivalent to a Newtonian dynamics of the general structure'

$$
m \ddot{x}(t)=f\left(x(t), t,\left[x\left(t^{\prime}\right)\right], \phi_{0}\right) .
$$

In most cases, an explicit analytical expression for $f\left(x(t), t,\left[x\left(t^{\prime}\right)\right], \phi_{0}\right)$ is not available ${ }^{\mathrm{T}} \mathrm{since}$ this would require analytical solutions $x_{j}\left(t,\left[x\left(t^{\prime}\right)\right], \phi_{0}\right)$ of a high dimensional chaotic dynamics and would in fact comprise the derivation of the basic principles of equilibrium statistical mechanics as special case. Rather, one proceeds the other way round, exploiting the fact that the environment is a thermal equilibrium heat bath and thus statistical mechanical principles can be invoked. Namely, one assumes that the systems initial conditions $x(0)$ and $p(0)$ are arbitrary but fixed, while the initial state of the bath $\phi_{0}$ is randomly sampled from a canonical probability distribution ${ }^{\text {TI }}$ $P\left(\phi_{0}\right) \propto \exp \left\{-H\left(x(0), p(0), \phi_{0}\right) / k_{B} T\right\}$. It is via this randomness of the environmental initial conditions $\phi_{0}$ that the system dynamics (3.27) acquires itself a stochastic nature. Denoting the average over those initial conditions by

$$
\tilde{f}\left(x(t), t,\left[x\left(t^{\prime}\right)\right]\right):=\left\langle f\left(x(t), t,\left[x\left(t^{\prime}\right)\right], \phi_{0}\right)\right\rangle
$$

we can decompose the right hand side of (3.27) into a sum of three terms,

$$
m \ddot{x}(t)=-V^{\prime}(x(t))-h\left(x(t), t,\left[\dot{x}\left(t^{\prime}\right)\right]\right)+\xi\left(x(t), t,\left[x\left(t^{\prime}\right)\right], \phi_{0}\right),
$$

where the first term is determined by the instantaneous state of the system, the second by its past history, and the third term is of microscopic origin, giving rise to the stochastic nature of the dynamics. Their explicit definitions are:

$$
\begin{aligned}
& V^{\prime}(x(t)):=-\tilde{f}\left(x(t), t,\left[x\left(t^{\prime}\right) \equiv x(t)\right]\right) \\
& h\left(x(t), t,\left[\dot{x}\left(t^{\prime}\right)\right]\right):=-\tilde{f}\left(x(t), t,\left[x\left(t^{\prime}\right)\right]\right)+\tilde{f}\left(x(t), t,\left[x\left(t^{\prime}\right) \equiv x(t)\right]\right) \\
& \xi\left(x(t), t,\left[x\left(t^{\prime}\right)\right], \phi_{0}\right):=f\left(x(t), t,\left[x\left(t^{\prime}\right)\right], \phi_{0}\right)-\tilde{f}\left(x(t), t,\left[x\left(t^{\prime}\right)\right]\right) .
\end{aligned}
$$

Here, $\left[x\left(t^{\prime}\right) \equiv x(t)\right]$ means that the function $x\left(t^{\prime}\right)$ keeps the same value $x(t)$ for all times $t^{\prime} \in[0, t]$ and is understood as a formal functional argument rather than an actual solution of the real system dynamics (3.29). Further, the modified functional argument $\left[\dot{x}\left(t^{\prime}\right)\right]$ on the left hand side of (3.31) is justified by the fact that any function $x\left(t^{\prime}\right)$ with $t^{\prime} \in[0, t]$ can be reconstructed from the knowledge of $x(t)$ and $\dot{x}\left(t^{\prime}\right)$. Finally, we remark that the souce of randomness $\phi_{0}$ enters via the "noise" (3.32), which has a vanishing mean value by construction.

\footnotetext{
${ }^{9}$ The explicit but formal expression of $f\left(x(t), t,\left[x\left(t^{\prime}\right)\right], \phi_{0}\right)$ in terms of the potentials in (3.26) and the formal solutions $x_{j}\left(t,\left[x\left(t^{\prime}\right)\right], \phi_{0}\right)$ is straightforward but of no further use, see below. Especially, $f\left(x(t), t,\left[x\left(t^{\prime}\right)\right], \phi_{0}\right)$ in (3.27) has nothing to do with $f(t)$ from (3.1).

${ }^{10}$ The only solvable exception - the so-called harmonic oscillator bath - arises when $V_{b}\left(x, x_{1}, \ldots, x_{N}\right)$ in 3.26 is a quadratic function of its arguments and thus the bath-dynamics is not chaotic, see section 8.1 .

${ }^{1}$ The physical origin of this canonical description is a "superbath" to which the bath of actual interest is weakly coupled.
} 
Observing that for $x\left(t^{\prime}\right) \equiv x(t)$ the bath keeps its initial canonical probability distribution and expressing the force on the right hand side of (3.27) in terms of the potentials in (3.26) one can infer from (3.28) and (3.30) that

$$
V(x)=V_{s}(x)-k_{B} T \ln \int \prod_{j=1}^{N} d x_{j} \exp \left\{-V_{b}\left(x, x_{1}, \ldots, x_{N}\right) / k_{B} T\right\} .
$$

In general, the bare system potential is thus renormalized (dressed) by the eliminated degrees of freedom of the environment and plays a role similarly to a free energy rather than a (bare) energy $92,93,150,186]$. However, if the potential $V_{b}\left(x, x_{1}, \ldots, x_{N}\right)$ is translation invariant (i.e. equal to $V_{b}\left(x+\Delta, x_{1}+\Delta, \ldots, x_{N}+\Delta\right)$ for all $\Delta$ ) then the renormalization in (3.33) boils down to an irrelevant additive constant.

\section{Linearized friction and thermal fluctuations}

While all so far formal manipulations are still exact, we finally make two approximations with respect to the "friction" term (3.31). First, we functionally expand $h\left(x(t), t,\left[\dot{x}\left(t^{\prime}\right)\right]\right)$ with respect to $\dot{x}\left(t^{\prime}\right)$. Considering that $h\left(x(t), t,\left[\dot{x}\left(t^{\prime}\right) \equiv 0\right]\right)=0$ (cf. (3.31)) and that $t^{\prime} \in[0, t]$, the leading order approximation is

$$
h\left(x(t), t,\left[\dot{x}\left(t^{\prime}\right)\right]\right) \simeq \int_{0}^{t} d s \frac{\delta h\left(x(t), t,\left[\dot{x}\left(t^{\prime}\right) \equiv 0\right]\right)}{\delta \dot{x}(s)} \dot{x}(s) .
$$

Second, we exploit the assumed property that the relevant state variable $x(t)$ changes "slowly" in comparison with the environment, hence $\dot{x}(s) \simeq \dot{x}(t)$ for all $s$-values which notably contribute in (3.34) (Markov approximation). By closer inspection one sees that within the same approximation the remaining intergal does no longer explicitly depend on $t$. As a result, we approximately find a friction term of the following general form

$$
h\left(x(t), t,\left[\dot{x}\left(t^{\prime}\right)\right]\right) \simeq \eta(x(t)) \dot{x}(t) .
$$

As far as the omitted corrections on the right hand side of (3.35) are not incidentally identically zero, by neglecting them we are tampering with the original equilibrium environment with the consequence of a (possibly very small but generically non-vanishing) breaking of thermal equilibrium and thus a violation of the second law of thermodynamics, see also sections 2.9 and 8.1. This shortcoming can only be remedied by a corresponding adjustment of the fluctuations in (3.32) in the following way: Along a similar line of reasoning as in 97 (see also section A.1 in Appendix A) one can show that the specific structure (3.29), (3.35) of the dynamics together with the requirement that the environment is at thermal equilibrium (respects the second law of thermodynamics) uniquely determine all statistical properties of those properly adjusted fluctuations appearing in (3.29). Namely, they are necessarily an unbiased Gaussian white noise whose correlations satisfy a fluctuation-dissipation relation of the form

$$
\left\langle\xi\left(x(t), t,\left[x\left(t^{\prime}\right)\right], \phi_{0}\right) \xi\left(x(s), s,\left[x\left(s^{\prime}\right)\right], \phi_{0}\right)\right\rangle=2 \eta(x(t)) k_{B} T \delta(t-s) .
$$

As already noticed below (2.5), the function $\eta(x)$ may thus be viewed as the coupling strength to the thermal environment. 
If the potential $V_{b}\left(x, x_{1}, \ldots, x_{N}\right)$ is known to be translation invariant then not only the renormalization of the potential in (3.33) reduces to an irrelevant additive constant but also the spatial dependence of the friction coefficient $\eta(x)$ disappears. In the overdamped limit $m \rightarrow 0$ we thus exactly recover our "unperturbed" working mode ${ }^{12}$ (3.1), (3.2). This omission of the inertia term in (3.1) is usually a quite satisfactory approximation for the typically very small systems under consideration, cf. section A.4 in Appendix A. A noteworthy exception is the case of a SQUID system[3], for which both the overdamped limit (3.1) as well as the case with finite inertia describe realistic experimental situations of interest, see section 5.6.

The translation invariance of $V_{b}\left(x, x_{1}, \ldots, x_{N}\right)$ and thus the $x$-independence of the system-bath coupling $\eta$ arises naturally if the periodic potential in (3.1) and the thermal environment have different physical origins. Since this is the case in most concrete examples which we will consider or at least it can be assumed without missing basic new effects, we will mostly focus on an $x$-independent friction coefficient $\eta$ henceforth. Prominent examples with $x$-dependent friction coefficients $\eta(x)$ are discussed in sections 6.4 .2 and 7.3 .

One basic assumption in our so far discussion has been the existence of a clear-cut separation between the characteristic time scales governing the "slow" system variable and those of the environment, with the consequence of a memoryless friction mechanism and uncorrelated thermal fluctuations. However, there exist physical systems for which this assumption is not fulfilled. One reason may be that one has overlooked additional relevant "slow" state variables and thus one simply has to go over to a higher dimensional vector $x(t)$ in the above calculations. However, in some cases the necessary dimensionality of $x(t)$ may become very high, while those additional dimensions are actually of no further interest, so that keeping a memory-friction and correlated noise may be more convenient. Restricting ourselves to the simples case with a translation invariant potential $V_{b}\left(x, x_{1}, \ldots, x_{N}\right)$, the approximation (3.34) takes the general form

$$
h\left(x(t), t,\left[\dot{x}\left(t^{\prime}\right)\right]\right) \simeq \int_{-\infty}^{t} d s \hat{\eta}(t-s) \dot{x}(s),
$$

where we have assumed that $\dot{x}(t)=0$ for all $t \leq 0$ in order to uniquely define the evolution of the integro-differential equation (3.29), and hence the lower integration limit could been extended to $-\infty$. Similarly as in (3.36), the assumption of thermal equilibrium then implies that the properly adjusted fluctuations appearing in (3.29) are necessarily an unbiased Gaussian noise whose correlation satisfies a fluctuationdissipation relation of the form

$$
\left\langle\xi\left(x(t), t,\left[x\left(t^{\prime}\right)\right], \phi_{0}\right) \xi\left(x(s), s,\left[x\left(s^{\prime}\right)\right], \phi_{0}\right)\right\rangle=\hat{\eta}(t-s) k_{B} T .
$$

Examples of this type will be discussed in sections 6.4 .3 and 8.1 .

It should be emphasized that the dynamics $(3.29)$ reproduces the correct equilibrium distribution $P(x, p) \propto \exp \left\{-\left[p^{2} / 2 m+V(x)\right] / k_{B} T\right\}$ in the long time limit,

\footnotetext{
${ }^{12}$ For the sake of notational simplicity only we have not included $f(t), y(t)$, and $F$ into the definition of $V_{s}(x)$ form 3.26 .

${ }^{13}$ The reason is that the "effective inertia" in a SQUID has a "macroscopic" origin, namely the capacitance of the considered circuit, cf. section 5.6.

${ }^{14}$ This can be physically realized by means of a time dependent potential $V_{s}(x, t)$ in $(3.26)$ which keeps $x(t)$ at a fixed position for $t \leq 0$ and switches to the actual potential of interest for $t>0$.
} 
independently of the choice of $\eta(x)$ or $\hat{\eta}(t)$ in (3.35)-(3.38). Especially, this distribution is exactly identical to the steady state result for the original system (3.30)-(3.32) before making any approximations [93]. Moreover, the second law of thermodynamics is strictly satisfied in all cases. It is only away from equilibrium that the specific choice of $\eta(x)$ or $\hat{\eta}(t)$ becomes important ${ }^{[5}$ and that the approximations made in (3.35)-(3.37) may have a noticeable effect.

In general, the above program of identifying "slow" and "fast" variables, establishing the microscopic model (3.26), and determining $\square(x)$ or $\hat{\eta}(t)$ according to (3.34) cannot be practically carried out [93]. The same applies for a well-controlled justification of the approximation (3.34), although this linearization turns out to provide remakably good approximations in a large variety of different systems. One reason may be the fact that in most cases only terms of odd order in the system velocity will contribute to the omitted corrections on the right hand side of (3.34) due to symmetry reasons. In view of those practical difficulties we are thus in some sense back at a phenomenological modeling which draws its legitimation from the comparison with experimental findings. However, as already mentioned, the microscopic modeling provides a general framework (functional form) for a large class of approximate models and a feeling for their wide range of applicability as well as for possible reasons in case they fail.

\subsubsection{Nonequilibrium perturbations}

There are two main types of possible "perturbations" of the "unperturbed" equilibrium system (3.1) with $f(t) \equiv 0, y(t) \equiv 0$, and $F=0$. The first acts essentially like the force $F$ in (3.1), i.e. the system $x$ gains (or looses) energy if it is displaced by one spatial period $L$. For instance, this may be a homogeneous force acting on a true Brownian particle or an angular momentum-type perturbation if $x$ was originally of a phase-like nature. In any case, such a perturbation interacts directly with the state variable $x$. The unbiased, time-dependent part of such a perturbation gives rise to the "tilting force" $y(t)$ and the systematic part to the "static force" $F$ in (3.1). The second possible type of perturbations interacts directly with the system variable $x$ but does not lead to an energy change if $x$ is displaced by one period $L$. A simple example is an electrical dipole with a single rotational degree of freedom in a homogeneous electrical field.

Another option is a perturbation which does not directly interact with the state variable $x$, but rather affects the physical mechanism responsible for the periodic potential in (3.1). Either some "internal degree of freedom" of the system $x$ is excited, which modifies the interaction with the periodic potential [13], or the periodic potential itself may be affected by the perturbation [187]. For instance, an electrical field may change the internal charge distribution (electrical polarization) of a neutral Brownian particle or of the periodic substrate with which it interacts. This type of perturbation gives rise to a "pulsating potential" $V(x, f(t))$ in (3.1). Depending on the details of the system, either one of the three basic types (fluctuating, improper traveling, or

\footnotetext{
${ }^{15}$ An obvious example is the mobility in the absence of the system potential $V_{s}(x)$, independently of whether the system is close to or far from equilibrium. Other observables which significantly depend on the choice of $\eta(x)$ or $\hat{\eta}(t)$ are escape rates (even so-called equilibrium rates) [66], as well as the particle current and the effective diffusion from (3.4) and (3.6).

${ }^{16}$ The explicit detemination of $V(x)$ according to 3.33 may still be feasible.
} 
genuine traveling potential) arises in its pure form, or a combination thereof, possibly even with a tilting ratchet admixture, is encountered.

One possible origin of those different types of "perturbations" may be an experimentally applied external field. While periodic signals then clearly represent the standard case, random perturbations have been realized as well [188]. Another possibility is a system-intrinsic source of "perturbations", usually of stochastic nature. The origin of such an intrinsic noise source may be either a non-equilibrium heat bath or a second thermal heat bath $\Pi$ at a different temperature than the $\xi(t)$-bath. As far as the tilting ratchet scheme is concerned, the coexistence of such an extra heat bath and the thermal $\xi(t)$-bath, which both interact directly with the state variable $x$ but practically not with each other, may be experimentally tailored, but is not very common in natural systems. Exceptions are electrical circuits, where non-equilibrium fluctuations, e.g. dichotomous noise [193] or shot noise (see Vol.1 of [115]) may naturally arise, and experimental analog electronic circuits for dichotomous [194, 195] or Gaussian 196 colored noise. More common are sources of noise which manifest themselves via an internal degree of freedom and thus lead to a pulsating ratchet scheme. Examples are catalytic chemical reactions with reactant and product concentrations far from their equilibrium ratio, or excitations induced by electromagnetic irradiation. Another example is a modified Feynman ratchet as discussed in section 6.2. In such cases, the coexistence of two practically independent sources of the noises $\xi(t)$ and $f(t)$ in (3.1) is indeed realistic.

At first glance, the property (3.3) that the potential is changing its shape in perfect synchrony over arbitrary distances $x$ might appear somewhat strange. However, this is in fact very natural if either $x$ is of a phase-like character or if the pulsating potential mechanism is caused by an internal degree of freedom of the system $x$. Also experimentally imposed external perturbations usually do not cause an asynchronous pulsating potential scheme. Asynchronously pulsating potentials 197 202 can only be expected if $x$ is a space-like variable and if the potential is subjected to independent "local" nonequilibrium noise sources, or in a specifically tailored experimental setup.

In the case of stochastic "perturbations" $f(t)$ or $y(t)$ in (3.1), we have assumed stationarity and especially $x$-independence of their statistical properties. Similarly as for the thermal noise $\xi(t)$, this reflects the assumption that their origin is a "huge" heat bath which is practically not influenced by the behavior of the "small" system $x(t)$. A more drastic assumption in (3.1) is the implicit omission of a back-coupling mechanism ("active decoupling") [11, 12, 15] to the $f(t)$ - or $y(t)$-heat bath, analogous to the dissipation mechanism in the case of the equilibrium $\xi(t)$-bath. This means that the coupling to this former bath is very weak and that this bath is very far away ("highly excited") from equilibrium with respect to the $\xi(t)$-bath at temperature $T$. Only then, the effect of the fluctuations $f(t)$ or $y(t)$ are still appreciable while the corresponding back-coupling effects are negligible 18 . In chapter 0 we will encounter a

\footnotetext{
${ }^{17}$ Microscopic models for two (or more) coexisting thermal heat baths at different temperatures have been discussed in 189 192. In the case of a tilting ratchet scheme it turns out that a ratchet effect (cf. section 2.6.2) is only possible for a correlated (non-white) thermal noise $y(t)$ and a concomitant memory friction term, see section 6.4.3. A generalization of these microscopic models to the case that one bath is out of equilibrium is also possible.

${ }^{18}$ For example, the origin of $f(t)$ or $y(t)$ may be a second thermal equilibrium bath at a temperature much higher than $T$. Though such a model may not be very realistic it is of great conceptual appeal as one of the simplest models for a system far from equilibrium 189]: Two thermal equilibrium baths are connected through a single degree of freedom $x(t)$ and can be exploited to do work. A concrete
} 
specific model where such a back-coupling mechanism is fully taken into account (see section 7.3.1). Furthermore, it will be demonstrated explicitly how this back-coupling may again become negligible as the corresponding source of noise is driven far away from equilibrium (see section 7.4.2). Another example with a nontrivial back-coupling appears in section 6.2.

Note that if $f(t)$ or $y(t)$ are such that a periodic perturbation of the system arises, then those back-coupling effects are also omitted in our model (3.1) but in this case such an omission is very common. The actual justification for doing so, however, follows in fact along the same line of reasoning as for random perturbations!

In all those various cases, it is clear that the system can never reach a thermal equilibrium state even in the long time limit: either this is prohibited by a permanent periodic perturbation or a second heat bath out of equilibrium or at equilibrium but with a temperature different from $T$. In either case, the second law of thermodynamics cannot be applied, i.e. the symmetry of detailed balance is violated. In the absence of any other prohibitive symmetries, which we have systematically broken by our classification scheme (cf. section 3.3), we thus expect the generic occurrence of the ratchet effect $\langle\dot{x}\rangle \neq 0$ according to Curie's principle. The corresponding intuitive microscopic picture is a permanent energy flow from the source of the perturbations $f(t)$ or $y(t)$ - be it a periodic external driving or a second heat bath - into the thermal bath at temperature $T$ via the single common degree of freedom $x(t)$.

\subsection{Supersymmetry}

In this section we continue our symmetry considerations from section 3.2, where we have seen that breaking thermal equilibrium, or equivalently, breaking the symmetry of detailed balance in whatever way, in a periodic, asymmetric system, is generically sufficient for the ratchet effect to manifest itself: In general, the occurrence of a finite current in such systems is the rule rather than the exception, in accord with Curie's principle. We thus more and more return to Smoluchowski and Feynman's point of view that away from thermal equilibrium, the absence rather than the presence of directed transport in spite of a broken symmetry is the truly astonishing situation. In this section, an entire class of such intriguing exceptional cases is identified which do not exhibit a ratchet effect in spite of broken thermal equilibrium and broken symmetry. Especially, such systems (cf. equations (3.1), (3.2) with $F=0$ ) exhibit zero current $\langle\dot{x}\rangle$ for any choice of the friction $\eta$, the temperature $T$, the amplitude and characteristic time scale of the drivings $f(t)$ and $y(t)$ etc., much like the symmetric systems from section 3.2. In contrast to usual current inversions (cf. sections 2.11 and 3.6), no fine-tuning of those parameters is thus required in order that $\langle\dot{x}\rangle=0$.

\subsubsection{Definitions}

We begin with the following definitions: We call a potential $V(x, f(t))$ with a periodic function $f(t)$ supersymmetric if there exist $\Delta x, \Delta t, \Delta V$ such that $-V(x, f(t))=$ $V(x+\Delta x, f(-t+\Delta t))+\Delta V$ for all $x$ and $t$. If $f(t)$ is a stochastic process then we call the potential $V(x, f(t))$ supersymmetric if for any $x$ all statistical properties of $-V(x, f(t))$ and $V(x+\Delta x, f(-t))+\Delta V$ are the same (no $\Delta t$ is needed since $f(t)$ is stationary).

example is the Feynman ratchet in section 6.2 . 
Especially, a static potential is supersymmetric if $-V(x)=V(x+\Delta x)+\Delta V$ for all $x$. Note that while we can and will choose the $t$ - and $V$-origins such that $\Delta t=$ 0 and $\Delta V=0$, the same is not possible for $\Delta x$. In fact, by applying the above defined supersymmetry transformation twice, we can conclude that $V(x+2 \Delta x, f(t))=$ $V(x, f(t))$ for all $x$ and $t$. Under the assumption that $L$ is the fundamental period of $V(x, f(t))$, i.e. the smallest $z>0$ with $V(x+z, f(t))=V(x, f(t))$, we can henceforth focus on $\Delta x=L / 2$. In summary, the supersymmetry criterion can thus be symbolically indicated (cf. (3.18)) for both, periodic and stochastic $f(t)$ as

$$
-V(x, f(t)) \hat{=} V(x+L / 2, f(-t)) .
$$

Turning to the driving $y(t)$, we will call it supersymmetric if for a periodic $y(t)$ we have that $-y(t)=y(-t+\Delta t)$ for all $t$ and an appropriate $\Delta t$, which can be transformed to zero as usual. For a stochastic $y(t)$ we speak of supersymmetry if $-y(t)$ and $y(-t)$ are statistically equivalent. In other words, supersymmetry means for both, periodic and stochastic $y(t)$, a parity-time-invariance of the form

$$
-y(t) \hat{=} y(-t)
$$

Regarding our above introduced notion of supersymmetry we remark that for undriven $(f(t) \equiv 0, y(t) \equiv 0, F=0)$ systems (3.1), a connection with supersymmetric quantum mechanics [203, 204] has been first pointed out in [205] and has been further developed in 206, 207], see also the [208] for a review. The basic idea is to transform the Fokker-Planck equation (cf. section 2.2) associated with the undriven stochastic dynamics (3.1) into a Schrödinger-type equation [99 101, 209, 210. By replacing in this equation the potential by its supersymmetric partner potential (in the quantum mechanical sense) a new Schrödinger equation emerges which can be transformed back into a new Fokker-Planck equation. The potentials of the original and the new FokkerPlanck equations then coincide (up to irrelevant shifts $\Delta x$ and $\Delta V$ ) if and only if the supersymmetry condition (3.39) is satisfied. In the presence of a periodic driving $y(t)$ (but still $f(t) \equiv 0, F=0$ ) in the stochastic dynamics (3.1), a similar line of reasoning has been developed in [211], yielding the supersymmetry condition (3.40). The case of various stochastic drivings $y(t)$ has been addressed in [212,213. Here, we will borrow the previously established notion of "supersymmetry" for the conditions (3.39), (3.49), but we will neither exploit nor further discuss their connection with quantum mechanical concepts.

\subsubsection{Main conclusion}

We now come to the central point of this section: We consider the general stochastic dynamics (3.1), (3.2) with $F=0$ together with the usual assumptions on $f(t)$ and $y(t)$ from section 3.1. By introducing $z(t):=x(-t)+L / 2$, we can infer that $\dot{z}(t):=-\dot{x}(-t)$, i.e. the averaged currents satisfy only deterministic and/or stationary stochastic processes appear in (3.1), (3.2), hence the evolution of the dynamics backward in time does not give rise to any problem.

\footnotetext{
${ }^{19}$ Note that it is not possible to derive this conclusion $\langle\dot{z}\rangle=-\langle\dot{x}\rangle$ from (3.5). The reason is that the initial and final times exchange their roles when going over from $x(t)$ to $z(t)$ and thus the implicit assumtion in (3.5) that the initial time is kept fixed while $t \rightarrow \infty$ is no longer fulfilled for $z(t)$. The properly generalized version of (3.5) reads $\langle\dot{x}\rangle=\lim _{t-t_{0} \rightarrow \infty}\left[x(t)-x\left(t_{0}\right)\right] /\left[t-t_{0}\right]$, from which one readily recovers $\langle\dot{z}\rangle=-\langle\dot{x}\rangle$.
} 
Especially, $-\xi(-t)$ is statistically equivalent to the forward Gaussian white noise $\xi(t)$. On the other hand, if both $V(x, f(t))$ and $y(t)$ are supersymmetric according to (3.39), (3.40) then one can readily see that $z(t)$ satisfies the same dynamics (3.1) as $x(t)$. Due to the self-averaging property of the current in (3.5) it follows that $\langle\dot{z}\rangle=\langle\dot{x}\rangle$. In view of our previous finding $\langle\dot{z}\rangle=-\langle\dot{x}\rangle$ we arrive at our main conclusion: if both $V(x, f(t))$ and $y(t)$ are supersymmetric according to (3.39), (3.49) then the average particle current $\langle\dot{x}\rangle$ is zero, see also [214 217].

We emphasize again that the conclusion $\langle\dot{x}\rangle=0$ only holds true if either both, the potential and the driving are symmetric or if both of them are supersymmetric. In any other case, $\langle\dot{x}\rangle \neq 0$ is expected generically. Especially, a symmetric but not supersymmetric potential in combination with a supersymmetric but not symmetric driving generically implies $\langle\dot{x}\rangle \neq 0$ (see sections 3.5 .3 and 5.8 for more details and examples).

\subsubsection{Examples}

Next we turn to the discussion of examples. Our first observation is the following completely general implication of the supersymmetry condition (3.39): For any minimum of $V(x, f(t))$, say at $x=x_{\min }$, there exists a corresponding maximum at $x=x_{\min }+L / 2$ and vice versa 20 . For the rest, the condition (3.39) is still satisfied by a very large class of potentials and their exhaustive characterization on an intuitive level seems rather difficult. Here, we restrict ourselves to two sufficient (but not necessary) simple criteria, which are still very general, namely:

1. The potential $V(x, f(t))$ is of the general form

$$
V(x, f(t))=\sum_{n=1,3,5, \ldots} \alpha_{n}(f(t)) \cos \left(\frac{2 \pi n x}{L}+\psi_{n}(f(t))\right)
$$

and $f(t)$ time-inversion invariant,

where time-inversion invariance of $f(t)$ means, in the same sense as in (3.40), that $f(-t) \hat{=} f(t)$. A typical example of this type (3.41) of supersymmetric potential $V(x, f(t))$ is depicted in figure 3.1. Note that in general not only the shape of $V(x, f(t))$ but also the location of the extrema may still be different for any $f(t)$-value. One readily sees that (3.41) indeed implies (3.39). For fluctuating potential ratchets, i.e. $V(x, f(t))=V(x)[1+f(t)]$, and especially for static potentials $V(x)$, also the inverse can be shown, that is, (3.41) is an exhaustive characterization of supersymmetric potentials in these special cases, but not in general.

2. A second class of supersymmetric potentials $V(x, f(t))$ is obtained by means of the representation

$$
\begin{aligned}
V(x, f(t)) & =V_{+}(x, f(t))+V_{-}(x, f(t)) \\
V_{ \pm}(x, f(t)) & :=\frac{V(x, f(t)) \pm V(x,-f(t))}{2}
\end{aligned}
$$

i.e., the potential is decomposed into symmetric and antisymmetric contributions with respect to $f(t)$,

$$
V_{ \pm}(x,-f(t))= \pm V_{ \pm}(x, f(t))
$$

\footnotetext{
${ }^{20}$ Since this property holds separately for any given $f(t)$-value, $x_{\min }$ and $x_{\max }$ may in general still depend on $t$.
} 


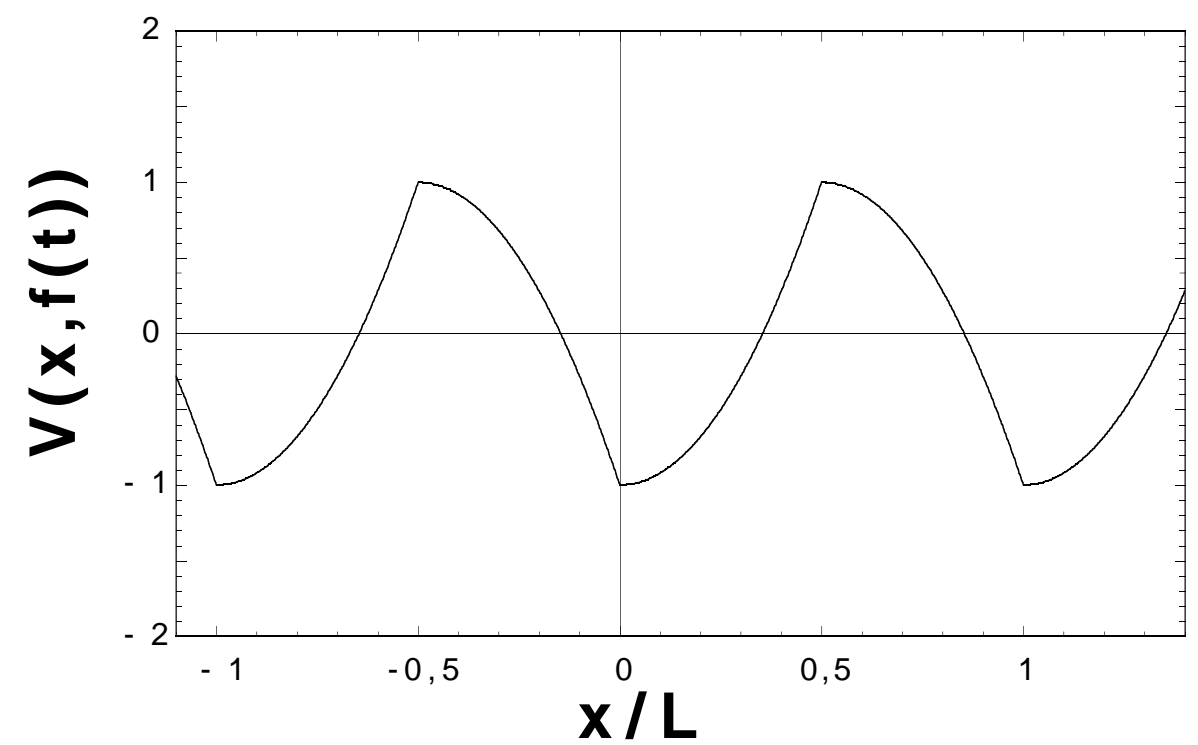

Figure 3.1: Example of a supersymmetric potential $V(x, f(t))$ (in arbitrary units) of the type (3.41) at an arbitrary but fixed $f(t)$-value.

Then, the following conditions are sufficient for the potential $V(x, f(t))$ to be supersymmetric:

$$
\begin{aligned}
& V_{+}(x, f(t))=\sum_{n=1,3,5, \ldots} \alpha_{n}(f(t)) \cos \left(\frac{2 \pi n x}{L}+\psi_{n}(f(t))\right) \\
& \text { and } V_{-}(x+L / 2, f(t))=V_{-}(x, f(t)) \\
& \text { and } f(t) \text { supersymmetric . }
\end{aligned}
$$

One readily verifies that these conditions (3.45) in combination with (3.42), (3.44) indeed imply (3.39), i.e. $V(x, f(t))$ is supersymmetric. A simple example is a supersymmetric $f(t)$ and

$$
V(x, f(t))=V_{1}(x)+V_{2}(x) f(t),
$$

where $V_{1}(x)$ is a static supersymmetric potential (cf. equation (3.41) and figure 3.1) and where $V_{2}(x)$ is an arbitrary $L / 2$-periodic function. In other words, in (3.42) the potential $V_{+}(x, f(t))$ is independent of $f(t)$ and $V_{-}(x, f(t))$ is linear in $f(t)$.

Next we come to the supersymmetry conditions 27 for the driving $y(t)$. If $y(t)$ is periodic then the condition (3.40) of supersymmetry is equivalent to a Fourier representation of the general form

$$
y(t)=\sum_{n=1}^{\infty} \gamma_{n} \sin (2 \pi n t / \mathcal{T}) .
$$

A typical example of such a supersymmetric $y(t)$ is depicted in figure 3.2. For a stochastic $y(t)$ we can rewrite $(3.40)$ as

$$
\left\langle y\left(t_{1}\right) y\left(t_{2}\right) \cdots y\left(t_{n}\right)\right\rangle=(-1)^{n}\left\langle y\left(-t_{1}\right) y\left(-t_{2}\right) \cdots y\left(-t_{n}\right)\right\rangle
$$

\footnotetext{
${ }^{21}$ They can of course be immediately transcribed into corresponding supersymmetry conditions for $f(t)$ as well.
} 


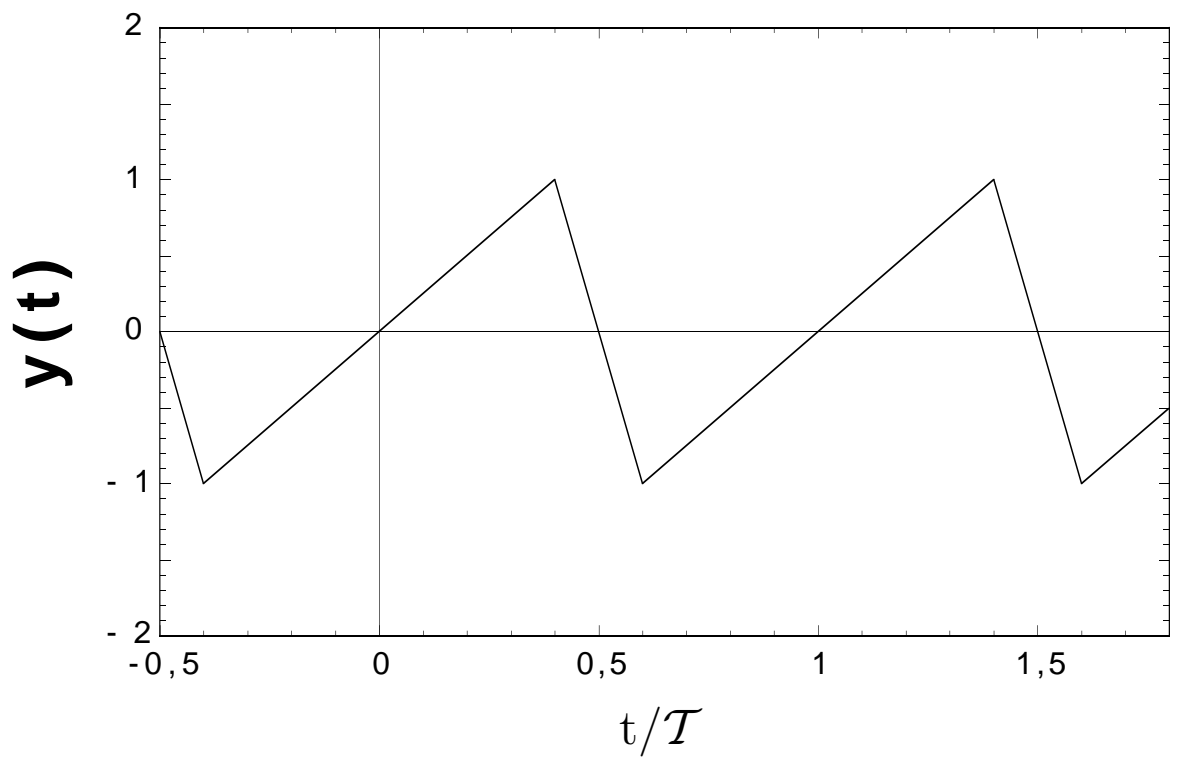

Figure 3.2: Example of a supersymmetric $\mathcal{T}$-periodic driving $y(t)$ (in arbitrary units) of the type (3.47).

for all integers $n \geq 1$ and all times $t_{1}, t_{2}, \ldots, t_{n}$ (see also the discussion below equation (3.21)). Note that out of the three possible symmetry properties of $y(t)$, namely (ordinary) symmetry, supersymmetry, and time-inversion invariance, two always imply the third. All three invariance properties are indeed satisfied for many particularly simple examples $y(t)$ which we will treat in more detail below, for instance symmetric dichotomous noise and Ornstein-Uhlenbeck noise (see section 3.1), as well as symmetric Poissonian shot noise (see section 5.1). Note also that arbitrary linear combinations of supersymmetric drivings are again supersymmetric.

A few specific examples which, prima facie quite astonishingly, produce zero current due to supersymmetry reasons are worth mentioning: The first set of examples are tilting ratchets $(f(t) \equiv 0)$ with a supersymmetric potential like in figure 3.1 and a periodic driving $y(t)$ like in (3.47), see also figure 3.2, or with a symmetric dichotomous noise $y(t)$, an Ornstein-Uhlenbeck noise $y(t)$, or a symmetric Poissonian shot noise $y(t)$. On the other hand, a symmetric, but not supersymmetric potential $V(x)$ (e.g. 3.19) with $a_{1} \neq 0$ and $\left.a_{2} \neq 0\right)$ in combination with a supersymmetric but not symmetric driving $y(t)$ (e.g. (3.47) with $\gamma_{1} \neq 0$ and $\gamma_{2} \neq 0$ ) does generically produce a finite current $\langle\dot{x}\rangle$, see section 5.8 .

A summary of the symmetry considerations for tilting ratchets with periodic drivings (i.e. rocking ratchets and asymmetrically tilting ratchets) is depicted in figure 3.3 . In order to bring out the essential features as clearly as possible, we have chosen in this figure stylized, non-smooth potentials $V(x)$ and drivings $y(t)$ and we have restricted ourselves to time-periodic $y(t)$.

In the case of a pulsating ratchet $(y(t) \equiv 0)$, a symmetric dichotomous noise $f(t)$, an Ornstein-Uhlenbeck noise $f(t)$, a symmetric Poissonian shot noise $f(t)$, or a periodic $f(t)$ of the form (3.47) yields $\langle\dot{x}\rangle=0$ if one of the following two conditions is met: (i) The potential $V(x, f(t))$ is for any given $f(t)$-value of the form (3.39), see also figure 3.1. We recall that not only the shape of $V(x, f(t))$ but also the location of 

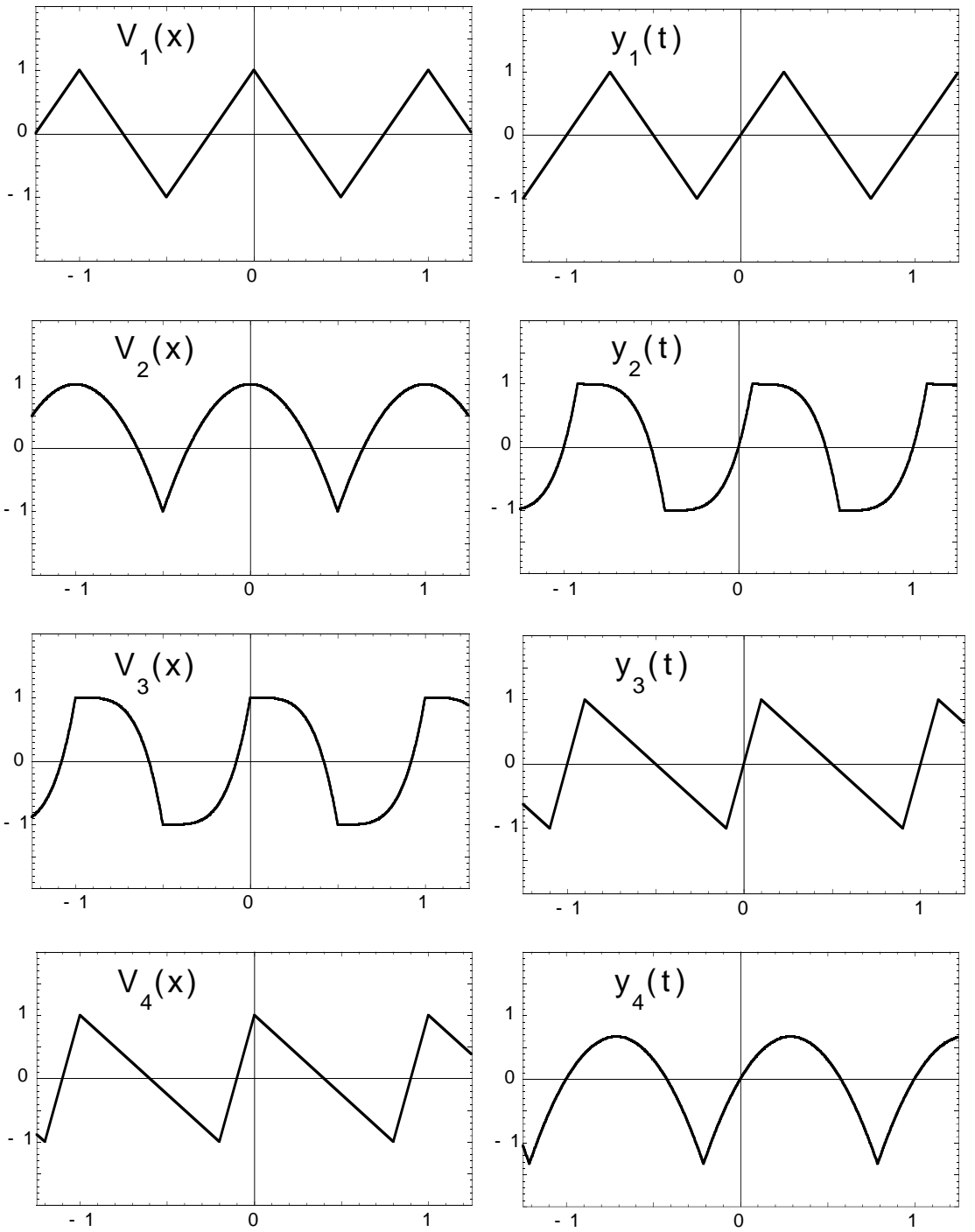

Figure 3.3: Summary of the symmetry considerations for tilting ratchets with potentials $V_{i}(x)$ and periodic drivings $y_{i}(t)$ (in arbitrary units). $i=1$ : symmetric and supersymmetric. $i=2$ : symmetric but not supersymmetric. $i=3$ : supersymmetric but not symmetric. $i=4$ : neither symmetric nor supersymmetric (but still satisfying (3.9)). The particle current (3.5) vanishes for arbitrary combinations of potentials and drivings which are either both symmetric or both supersymmetric. For any other combination of potentials and drivings, a finite current arises generically.

the extrema may be different for any $f(t)$-value, i.e. both fluctuating potential ratchet and (improper) traveling potential ratchets are covered. (ii) The potential $V(x, f(t))$ respects supersymmetry when $f(t)=0$ and is augmented for $f(t) \not \equiv 0$ by a fluctuating potential term $V_{2}(x) f(t)$ with an arbitrary $L / 2$-periodic function $V_{2}(x)$, see (3.46).

\subsubsection{Discussion}

As long as $V(x, f(t))$ and $y(t)$ are supersymmetric, the property $\langle\dot{x}\rangle=0$ is robust with respect to any change of the friction $\eta$, temperature $T$, amplitude and characteristic 
time scale of the drivings $f(t)$ and $y(t)$ etc. Much in contrast to ordinary current inversions, we thus find $\langle\dot{x}\rangle=0$ without fine-tuning any of these model parameters. The same is of course true for symmetric instead of supersymmetric $V(x, f(t))$ and $y(t)$. Note that this conclusion is no contradiction to Curie's principle since a generic variation within the entire class of admitted ratchet models also involves a change of $V(x, f(t))$ and $y(t)$ such that these symmetries are broken, see also the concluding discussion in chapter 10 .

In the above respect, but also upon comparison of (3.19)-(3.21) with (3.41), (3.47), (3.48), the formal structure and the consequences of symmetry and supersymmetry are remarkably similar. There is, however, also one fundamental difference which appears if an additional inertia term $m \ddot{x}(t)$ is included on the left hand side of the general ratchet dynamics (3.1): While symmetry implies $\langle\dot{x}\rangle=0$ even in the presence of inertia effects, the same conclusion no longer applies in the case of supersymmetry. For instance, a rocking ratchet with a cosine potential $V(x)$ and a driving $y(t)$ like in figure 3.2 implies $\langle\dot{x}\rangle=0$ in the overdamped limit [31,218,219] but generically $\langle\dot{x}\rangle \neq 0$ if inertia is included 220]. In the opposite limit of a deterministic Hamiltonian rocking ratchet dynamics (finite inertia, vanishing dissipation and thermal noise) a condition [221] reminiscent of supersymmetry will be discussed in section 5.4. In the intermediate regime of finite inertia and dissipation, no comparable symmetry concept is known. Since the current changes always continuously upon variation of any model parameter, it follows that for any sufficiently small deviations from a perfectly supersymmetric situation, e.g. in the presence of a very small ineria term, the current $\langle\dot{x}\rangle$ will still be arbitrarily small 2215. In the following we focus again on the overdamped limit.

\subsubsection{Generalizations}

We close with a brief look at the ratchet classes with both $f(t) \equiv 0$ and $y(t) \equiv 0$ in (3.1) but instead with a varying temperature $T$ in (3.2): In the case of Seebeck ratchets, characterized by a space dependent, $L$-periodic temperature $T(x)$, we speak of a (spatially) symmetric system if both, $V(x)$ and $T(x)$ satisfy the symmetry condition (3.16) with the same $\Delta x$, which may be transformed to zero as usual, i.e.

$$
V(-x)=V(x) \text { and } T(-x)=T(x) \quad \text { [symmetry] }
$$

Similarly, supersymmetry is defined by the following condition for the potential together with a modified such condition for the temperature:

$$
-V(x)=V(x+L / 2) \text { and } T(-x)=T(x+L / 2) \quad \text { [supersymmetry]. }
$$

Along the same line of reasoning as in section 3.2, i.e. by considering the mirror image $-x(t)$ of $x(t)$, one readily finds that the average current $\langle\dot{x}\rangle$ indeed vanishes if the symmetry conditions (3.49) are satisfied. On the other hand, by considering $z(t):=x(-t)+L / 2$, one verifies that $\langle\dot{x}\rangle=0$ if supersymmetry (3.50) is respected.

Finally, in the case of temperature ratchets, characterized by a time-dependent temperature $T(t)$, a zero current $\langle\dot{x}\rangle=0$ is recovered provided that either

$$
V(-x)=V(x) \quad[\text { symmetry }],
$$

independently of the properties of $T(t)$, or that

$$
-V(x)=V(x+L / 2)
$$

and $T(t)$ time-inversion invariant [supersymmetry] . 
Comparison with (3.16) and (3.41) confirms once more the similarity between pulsating ratchets and temperature ratchets (see also section 6.3).

Further generalization to higher dimensional systems are also possible but not further pursued here, see also section 9.2.3.

\subsection{Tailoring current inversions}

The argument which we have invoked at the end of section 2.11 can be considerably generalized as follows: We consider any ratchet model of the general form (3.1)-(3.3), usually (not necessarily ${ }^{22}$ with $F=0$ in (3.1), and possibly also with an $x$ - and/or $t$-dependent temperature $T$ in (3.2). Next we focus on an arbitrary parameter $\mu$ of the model and we prescribe an arbitrary reference value $\mu_{0}$. Under the only assumption that two potentials $V_{i}(x, f(t)), i=0,1$, with opposite currents $\langle\dot{x}\rangle$ at $\mu=\mu_{0}$ exist, we can then construct a third potential, say $V_{\lambda_{0}}(x, f(t))$, with the property that the current $\langle\dot{x}\rangle$ as a function of the parameter $\mu$ exhibits a current inversion at the prescribed reference value $\mu_{0}$.

The proof of this proposition is almost trivial. Namely, we define a set of potentials

$$
V_{\lambda}(x, f(t)):=\lambda V_{1}(x, f(t))+(1-\lambda) V_{0}(x, f(t)),
$$

parametrically dependent on $\lambda \in[0,1]$. In other words, the potentials $V_{\lambda}(x, f(t))$ continuously interpolate between the above defined two potentials $V_{i}(x, f(t)), i=0,1$, with opposite current directions at $\mu=\mu_{0}$. Under the tacit assumption that the current $\langle\dot{x}\rangle$ changes continuously upon variation of $\lambda$, it follows that it vanishes at a certain intermediate potential $V_{\lambda_{0}}(x, f(t))$. We remark that this assumption is very weak: For instance, one can show that a non-vanishing thermal noise $\xi(t)$ in (3.1) is sufficient, but by no means necessary. Since the sign of $\langle\dot{x}\rangle$ is robust against small changes of $V_{i}(x, f(t))$, it can furthermore be taken for granted that $V_{\lambda_{0}}(x, f(t))$ is a generic potential in the sense that the dynamics (3.1) is neither symmetric nor supersymmetric, nor exhibits any other "accidental" symmetry. In other words, we are dealing with the generic case that, upon variation of the parameter $\mu$, the current $\langle\dot{x}\rangle$ exhibits an isolated zero, i.e. a genuine current inversion, at $\mu=\mu_{0}$.

If the condition that two potentials $V_{i}(x, f(t))$ with opposite current directions at $\mu=\mu_{0}$ exist is not fulfilled, then also a current inversion at $\mu_{0}$ is obviously not possible, i.e. this condition is both necessary and sufficient. For instance, if the driving $y(t)$ is symmetric and the temperature $T$ independent of $x$ then we know that $V(-x, f(t))$ yields a current opposite to that associated with $V(x, f(t))$. Hence we can choose as $V_{0}(x, f(t))$ any potential with $\langle\dot{x}\rangle \neq 0$ and as $V_{1}(x, f(t))$ a slightly deformed modification of $V_{0}(-x, f(t))$ to conclude that a current inversion exists always.

We may also consider some characteristic property of the driving $y(t)$ as variable and instead leave all the other ingredients (especially the potential) of the ratchet dynamics (3.1) fixed. If the existence of two special drivings $y_{i}(t), i=0,1$, with opposite currents at $\mu=\mu_{0}$ is known, then we can prove along the same line of reasoning as above that there exists at least one $\lambda \in(0,1)$, say $\lambda_{0}$, such that

$$
y_{\lambda}(t):=\lambda y_{1}(t)+(1-\lambda) y_{0}(t)
$$

\footnotetext{
${ }^{22}$ Note that current inversions upon variation of any model parameter can obviously be enforced by applying an appropriately chosen external force $F$ [54, 195, 222, 223.
} 
produces a current inversion at the arbitrarily prescribed reference parameter value $\mu_{0}$. For instance, if $V(x, f(t))$ is symmetric (and the temperature $T$ constant) then we know that an asymmetric $y(t)$ generically produces a current $\langle\dot{x}\rangle \neq 0$ and $-y(t)$ a current in the opposite direction. Hence, an appropriate asymmetric driving $y(t)$ can be tailored which separates particles on opposite sides of an arbitrarily prescribed parameter value $\mu_{0}$. Since $y(t)$ is typically generated by an externally applied field, such a separation scheme may be of considerable experimental interest.

At this point it is worth recalling that once a current inversion upon variation of one model parameter has been established, the existence of an inversion upon variation of any other parameter follows along the same line of reasoning as in section 2.11.

Current inversions upon changing certain parameters of the system have been studied for the first time in the context of photovoltaic effects in non-centrosymmetric materials 159, 160]. Early observations in simple theoretical models as we study them here are due to [35, 37, 39, 42]. Since then the search and control of current inversions has been attracting much attention with respect to the possibility of new particle separation technologies based on the ratchet effect. Moreover, multiple current inversions have been exemplified e.g. in 170,223 232. In the latter case, particles with parameter values within a characteristic "window" may be separated from all the others. The first systematic investigation of such multiple inversions from [232] suggest that it may always be possible to tailor an arbitrary number of current inversions at prescribed parameter values. However, a corresponding generalization of our rigorous proof has not yet been established.

Our method of tailoring current inversions implies that, in general, the direction of the particle current, and even more so its quantitative magnitude, depends in a very complicated way on many details of the ratchet potential $V(x, f(t))$ and/or on the driving $y(t)$. In this respect, the leading order small- $\mathcal{T}$ behavior of the temperature ratchet in (2.58) is still a rather simple example. Therefore, any heuristic "explanation" or simple "rule" regarding current directions should make us suspicious unless it is accompanied by a convincing (and usually rather severe) restriction on the admitted potentials $V(x, f(t))$ and/or the driving $y(t)$. Otherwise, one can typically construct even quite innocent looking counter-examples of such a "rule".

The above described procedure is a very simple and universal tool for the construction of current inversions per se. However, little control over the more detailed dependence of the current as a function of the considered parameter $\mu$ is possible in this way. For instance, the maximal magnitudes of the currents may be very different in the positive and negative directions. Likewise, we can hardly avoid ending up with a quite complicated looking potential $V_{\lambda_{0}}(x)$ and/or driving $y_{\lambda_{0}}(t)$. For both purposes, "symmetrically" shaped current inversions as well as "simple" potentials and/or drivings which do the job, more detailed analytical predictions are invaluable. For instance, the results depicted in figure 2.9 have not been obtained directly by the above construction scheme. Rather, the approximation (2.58) has been exploited in order to obtain such an "innocent" looking ratchet potential with a current inversion.

\subsection{Linear response and high temperature limit}

For vanishing $f(t), y(t)$, and $F$ we recover a Smoluchowski-Feynman ratchet in (3.1), yielding zero current (3.5) in the long time limit (steady state), see sections 2.1 2.4. In the case of a tilting ratchet scheme, an interesting question regards the linear response 
behavior in the presence of a weak but finite driving $y(t)$ (while $f(t)$ and $F$ are still zero), i.e. the behavior of the averaged long-time current (3.5) in linear order $23 y(t)$. In the case of a symmetric driving $y(t)$ (cf. (3.17) and (3.18)) no such linear contribution can arise, since the averaged long-time current is clearly invariant under inversion of the driving amplitude, $y(t) \mapsto-y(t)$. In the general (asymmetric) case, we can expand $y(t)$ into a Fourier series, which does not exhibit a constant term since $y(t)$ is unbiased, see (3.9) or (3.10). In linear order $y(t)$, the net current follows simply by summing up all the contributions of the single Fourier modes. Since each Fourier mode corresponds to a symmetric driving, the resulting net current is zero. A similar line of reasoning applies for pulsating ratchets with a weak driving $f(t)$ (and $y(t)=F=0$ ). An exception is a genuine traveling potential scheme with a systematic long time drift $u:=\lim _{t \rightarrow \infty} f(t) / t$. In this case, the above Fourier expansion of $f(t)$ cannot be applied any more and indeed a finite linear order $f(t)$ contribution to the current is observed generically, see section 4.4.1. In other words, for tilting ratchets, fluctuating force ratchets, and improper traveling potential ratchets no directed current occurs within the linear response regime (linear order $y(t)$ and $f(t)$, respectively). The same conclusions obviously extends to systems with simultaneously small but finite $y(t)$ and $f(t)$ (but still $F=0$ ). Due to their equivalence with fluctuating potential ratchets, the conclusion also carries over to temperature ratchets (cf. section 6.3) with a small perturbation of the temperature $T(t)$ about the (finite) average value $\bar{T}$.

In the above line of reasoning we have tacitly assumed analyticity of the current with respect to the amplitude of the perturbations $y(t)$ and $f(t)$ and that a Fourier expansion ${ }^{2}$ of these perturbations is possible. (Especially, for a stochastic process, the word "weak perturbation" refers to its intensity, but not necessarily to its instantaneous value at any given time $t$.) Though this may be difficult to rigorously justify in general, a more careful analysis of each specific case (known to the present author) shows that the conclusion of vanishing linear response remains indeed correct.

Another limit which admits a completely general conclusion is that of asymptotically large temperatures $T$ in (3.1), (3.2): Again one finds that the current $\langle\dot{x}\rangle$ always approaches zero in the limit $T \rightarrow \infty$. While this result is physically rather suggestive (the effect of the potential $V(x, f(t))$ is completely overruled by the noise $\xi(t)$ ) the technical details of the mathematical proof go beyond the scope of this review. In those numerous cases for which the current also vanishes for $T \rightarrow 0$, a bell-shaped $\langle\dot{x}\rangle$-versus- $T$ curve is thus recovered.

We finally remark that in the limit of asymptotically strong drivings $y(t)$ and/or $f(t)$, no generally valid predictions are possible.

\subsection{Activated barrier crossing limit}

For many of the above defined classes of ratchets (3.1) it may turn out that in the absence of the thermal Gaussian noise $(T=0)$, the particle $x(t)$ is confined to a restricted part of one spatial period for all times. In the presence of a small amount of thermal noise, the particle will be able to cross the previously forbidden regions by thermal activation. Yet, such events will be rare and after each thermally activated

\footnotetext{
${ }^{23}$ Formally, this amounts to replacing $y(t)$ by $\epsilon y(t)$ and then performing a series expansion in $\epsilon$ while keeping $y(t)$ fixed.

${ }^{24}$ Or any other series expansion in terms of symmetric basis functions.
} 
transition from one spatial period into an adjacent one, the particle will again remain there for a long time. Since the duration of the actual transition events is negligible in comparison with the time the particle spends in a quasi steady state (metastable state) between the transitions, it follows that the probability for a transition per time unit can be described in very good approximation by a constant rate 2 .

Denoting the rate for a transition to the right by $k_{+}$and to the left by $k_{-}$, the average particle current readily follows 20 as

$$
\langle\dot{x}\rangle=L\left[k_{+}-k_{-}\right] \text {. }
$$

For a special case, this result has been derived explicitly already in equation 2.44). Exploiting the rate description for the transitions once more, also the effective diffusion coefficient (3.6) can be readily evaluated [100] with the result

$$
D_{\mathrm{eff}}=\frac{L^{2}}{2}\left[k_{+}+k_{-}\right] .
$$

The very same conclusions (3.55), (3.56) hold true if transitions are not excluded but still very rare at $T=0$. This may be the case for instance in a tilting ratchet $(f(t) \equiv 0)$ when $y(t)$ is a Gaussian random process with a small intensity $\int d t\langle y(t) y(0)\rangle$. On the other hand, genuine traveling potential ratchets will turn out to support an appreciable particle current typically even for $T=0$ and are henceforth excluded.

The evaluation of the current (3.55) and the diffusion coefficient (3.56) has thus been reduced to the determination of certain rates $k$ across rarely visited regions between some type of effective local potential wells or periodic attractors (if $f(t)$ or $y(t)$ is periodic in $t$ ). In the case that both $f(t)$ and $y(t)$ are stochastic processes (possibly one of them identically zero), this problem of thermally activated surmounting of a potential barrier with randomly fluctuating shape has attracted considerable attention since the discovery of the so-called resonant activation effect [238], see [68] for a review. On condition that a rate description of the barrier crossing problem is possible, i.e. the transitions are rare events especially in comparison with the time scale of the barrier fluctuations 68], the fluctuating barrier crossing problem is thus equivalent to determining the current and diffusion in a ratchet model. A large body of analytical results on the former problem 239 254 are thus readily applicable for our present purposes. Particularly closely related to the resonant activation effect are the theoretical works [158,255] on externally driven molecular pumps (cf. section 4.6.1) and their experimental counterparts in 256, 257].

If $f(t)$ and/or $y(t)$ are periodic in time with a large period $\mathcal{T}$ then a close connection to the phenomenon of stochastic resonance [62] can be established. If the time period $\mathcal{T}$ is not very large, then this problem of thermally activated escape over an oscillating potential barrier represents a formidable technical challenge [258]. The few so far available results on weak [259, 260], slow (but beyond the adiabatic approximation)

\footnotetext{
${ }^{25}$ If $f(t)$ or $y(t)$ is periodic in time, then we will have a quasi periodic behavior between transition events and the transition probability is only given by a constant after "coarse graining" over one time-period.

${ }^{26}$ We tacitly focus here on the simplest and most common case with just one metastable state per period $L$. For more general cases, the current and the effective diffusion coefficient can still be expressed in closed analytical form in terms of all the involved transition rates, but the formulas become more complicated, see 233235 and further references therein. For special cases, such formulas have been repeatedly re-derived, e.g. in 236,237.
} 
[261], fast 262], and general oscillations [263,264] can again be readily adapted for our present purposes via (3.55), (3.56).

One basic conclusion of all the above mentioned analytical studies of the thermally activated escape over fluctuating or oscillating barriers is that those rates become exponentially small with decreasing thermal noise strength, much like in the simple explicit example (2.45), (2.46). On top of that, also the ratio $k_{+} / k_{-}$generically becomes either exponentially small or large. In other words, practically only transitions in one direction occur in the course of time and $x(t)$ basically simplifies to a unidirectional Poissonian hopping process [100]. This type of unidirectionality is a distinct weak-noise feature. As soon as the thermal noise-strength increases, the stochastic trajectory $x(t)$ always displays appreciable displacement in both directions.

Though a substantial part of the present author's contributions to the field under review is concerned with the evaluation of such thermally activated rates over fluctuating or oscillating barriers, we desist from going into any further details of these technically rather involved theories. 


\section{Chapter 4}

\section{Pulsating ratchets}

In this chapter we focus on the pulsating ratchet scheme, i.e., we consider a stochastic dynamics of the form

$$
\eta \dot{x}(t)=-V^{\prime}(x(t), f(t))+\xi(t)
$$

complemented by the fluctuation-dissipation relation (3.2) for the thermal noise $\xi(t)$ and the periodicity condition (3.3) for the potential. Further, $f(t)$ is assumed to be an unbiased time-periodic function or stationary stochastic process. As compared to the general working model (3.1) we have set the load force $F$ equal to zero on the right hand side of (4.1) since this case is usually of foremost interest.

\subsection{Fast and slow pulsating limits}

We consider an arbitrary (unbiased, i.e. $\langle f(t)\rangle=\langle f(0)\rangle=0)$ stochastic process $f(t)$ and assume that its stationary distribution $\rho(f)$ (see (3.11)) and thus especially its variance $\left\langle f^{2}(t)\right\rangle=\left\langle f^{2}(0)\right\rangle$ (see (3.14) ) is always the same, while its correlation time円

$$
\tau:=\frac{\int_{-\infty}^{\infty} d t\langle f(t) f(s)\rangle}{2\left\langle f^{2}(t)\right\rangle}
$$

characterizing the decay of the correlation $\langle f(t) f(s)\rangle=\langle f(t-s) f(0)\rangle$, can be varied over the entire positive real axis. More precisely, we assume that the process $f(t)$ can be written in the form

$$
f(t)=\hat{f}(t / \tau)
$$

where $\hat{f}(h)$ is a suitably defined, fixed reference process with dimensionless timeargument $h$, cf. (2.56). The statistical properties of the process $f(t)$ then depend solely on the parameter $\tau$, while $\rho(f)$ is $\tau$-independent. One readily sees that the examples of dichotomous and Ornstein-Uhlenbeck noise from (3.12)-(3.15) are of this type. This so-called constant variance scaling assumption [265] is "natural" in the same sense as it is natural to keep in a time-periodic perturbation $f(t)$ the amplitude fixed while the frequency is varied, cf. section 2.10 .

\footnotetext{
${ }^{1}$ By means of a calculation similar to that in section A.2 of Appendix A one can show that the intensity $\int d t\langle f(t) f(s)\rangle$ and hence the correlation time 4.2 is always non-negative.
} 
For very small $\tau$, the noise $f(t)$ changes very quickly, while its strength $\int d t\langle f(t) f(s)\rangle$ tends to zero (see also previous footnote 1 ). Thus, for $\tau \rightarrow 0$ (fast pulsating limit) there will be no effect of $f(t)$ in (4.1), i.e. we recover a SmoluchowskiFeynman ratchet with $\langle\dot{x}\rangle=0$. Similarly, for $\tau \rightarrow \infty$ the noise becomes very slow, $f(t) \simeq f=$ const. (adiabatic approximation, see also section 2.10). Since for any fixed value of $f$ we have again a Smoluchowski-Feynman ratchet in (4.1), the result $\langle\dot{x}\rangle=0$ subsists after averaging over all those fixed $f$-values according to $\rho(f)$. The very same conclusion $\langle\dot{x}\rangle=0$ holds for periodic functions $f(t)$ in the limits of asymptotically long and short periods $\mathcal{T}$ and can be furthermore extended also to generic traveling potential ratchets (cf. section 4.4.1). In other words, we arrive at the completely general result that for any type of pulsating ratchet ( 4.1 ), the current $\langle\dot{x}\rangle$ disappears both in the fast and slow pulsating limit.

We exemplify the leading order correction to this asymptotic result $\langle\dot{x}\rangle=0$ for the case that $f(t)$ is an arbitrary stationary stochastic process with very small correlation time $\tau$. Generalizing an argument form [68, 246] we can, within a leading order approximation, substitute in (4.1) the random process $V(x, f(t))$ for any fixed $x$ by an uncorrelated Gaussian process with the same mean value

$$
V_{0}(x):=\langle V(x, f(t))\rangle=\int_{-\infty}^{\infty} d f \rho(f) V(x, f)
$$

and the same intensity $\int d t C(x, t)$, where the correlation $C(x, t)$ is defined as

$$
C(x, t):=\left\langle V^{\prime}(x, f(t)) V^{\prime}(x, f(0))\right\rangle-\left[V_{0}^{\prime}(x)\right]^{2} .
$$

An alternative representation of $C(x, t)$ analogous to the second equality in (4.4) is given by

$$
C(x, t)=\int_{-\infty}^{\infty} d f_{1} d f_{2} \rho\left(f_{1}, f_{2}, t\right) V^{\prime}\left(x, f_{1}\right) V^{\prime}\left(x, f_{2}\right)
$$

where $\rho\left(f_{1}, f_{2}, t\right)$ is the joint two-time distribution of the stationary process $f(t)$, i.e.

$$
\rho\left(f_{1}, f_{2}, t\right):=\left\langle\delta\left(f(t)-f_{1}\right) \delta\left(f(0)-f_{2}\right)\right\rangle .
$$

Referring to [68, 246] for the calculational details, one obtains in this way the result

$$
\langle\dot{x}\rangle=\frac{L}{\eta^{2} k_{B} T} \frac{\int_{0}^{L} d x V_{0}^{\prime}(x) \int_{-\infty}^{\infty} d t C(x, t)}{\int_{0}^{L} d x e^{V_{0}(x) / k_{B} T} \int_{0}^{L} d x e^{-V_{0}(x) / k_{B} T}} .
$$

Note that the effective potential $V_{0}(x)$ is again $L$-periodic. Under the conditions that $T>0$ and that (4.5) decays exponentially in time, the result (4.8) gives the leading order contribution for small correlation times $\tau$ of $f(t)$. Using (4.3) we can infer that

$$
\int_{-\infty}^{\infty} d t C(x, t)=\tau \int_{-\infty}^{\infty} d h \hat{C}(x, h)
$$

where $\hat{C}(x, t)$ is defined like in 4.5$)$ but with $\hat{f}(h)$ instead of $f(t)$ and thus the integral on the right hand side of (4.9) is $\tau$-independent. In other words, the asymptotic current (4.8) grows linearly with the correlation time $\tau$. 
In the special case that $V(x, f(t))$ is of the fluctuating potential ratchet form $V(x)[1+f(t)]$, the small- $\tau$ result from [266] is readily recovered from (4.8), namely

$$
\langle\dot{x}\rangle=\frac{2 L \tau\left\langle f^{2}(t)\right\rangle \int_{0}^{L} d x\left[V^{\prime}(x)\right]^{3}}{\eta^{2} k_{B} T \int_{0}^{L} d x e^{V(x) / k_{B} T} \int_{0}^{L} d x e^{-V(x) / k_{B} T}},
$$

see also [267].

A similar leading order correction of the adiabatic approximation $\langle\dot{x}\rangle=0$ is possible for large $\tau$ [266] but leads to a complicated formal expression which still depends on much more details of the noise than in (4.8).

For periodic driving $f(t)$, expansions along the lines of Appendix $\mathrm{C}$ can be undertaken, see section 6.3. Technically similar calculations can also be found in 268,269 for fast and 261] for slow periodic driving. We will not pursue this task here any further[2] since the main conclusion follows from the already complicated enough result (4.8), namely that a finite current $\langle\dot{x}\rangle$ is generically expected for driving signals $f(t)$ with a finite characteristic time scale and that its sign and magnitude depends very sensitively on the details of the potential $V(x)$ and the driving $f(t)$.

\subsection{On-off ratchets}

The on-off ratchet scheme has been introduced in a specific theoretical context in 1987 by Bug and Berne [32] and has been independently re-invented as a general theoretical concept in 1992 by Ajdari and Prost [34. In its latter form it is of archetypal simplicity and the predicted occurrence of the ratchet effect has been verified by several experimental implementations. The model is a special case of the overdamped onedimensional Brownian motion dynamics (4.1), namely

$$
\eta \dot{x}(t)=-V^{\prime}(x(t))[1+f(t)]+\xi(t)
$$

where $V(x)$ is spatially periodic, asymmetric "ratchet"-potential. The function $f(t)$ is restricted to the two values \pm 1 , so the potential in (4.11) is either "on" or "off".

In the simplest case the potential $V(x)$ has one maximum and minimum per spatial period $L$ (for examples see figures 2.2 and 4.1 ), the potential difference between maxima and minima is much larger than the thermal energy $k_{B} T$, and $f(t)$ is a timeperiodic function with long sojourn-times in the +1-state (potential "on"). Under these premises the analysis of the model proceeds in complete analogy to figure 2.6. Qualitatively, a net pumping of particles into the positive direction will occur if the minima of $V(x)$ are closer to their neighboring maxima to the right than to the left ("forward on-off ratchet"), otherwise into the negative directionf. Quantitatively, the average current $\langle\dot{x}\rangle$ can be readily expressed in closed analytical form [34] apart from an error function, which has to be evaluated numerically. Similarly, one can readily

\footnotetext{
${ }^{2}$ In the special case of slow on-off and slow, traveling potential ratchets, the qualitative behavior will become intuitively clear later on.

${ }^{3}$ If $V(x, f(t))$ is of the fluctuating potential ratchet form $V(x)[1+f(t)]$ it is possible to show that $\langle\dot{x}\rangle$ vanishes faster than proportional to $\tau^{-1}$ in the slow driving limit $\tau \rightarrow \infty$ for both, periodic and stochastic $f(t)$. In the latter case, this conclusion is also contained implicitly in the calculations of 266 .

${ }^{4} \mathrm{~A}$ computer animation (Java applet) which graphically visualizes the effect is available on the internet under 270.
} 
evaluate [34] the effective diffusion coefficient (3.6). Depending on their friction coefficient $\eta$, different species of particles which are initially mixed (say $x(0)=0$ for all of them) will exhibit after a time $t$ different displacements $\langle\dot{x}\rangle t$ and dispersions $\sqrt{2 D_{\text {eff }} t}$ and thus can be separated for sufficiently large $t$, see also equation (3.7).

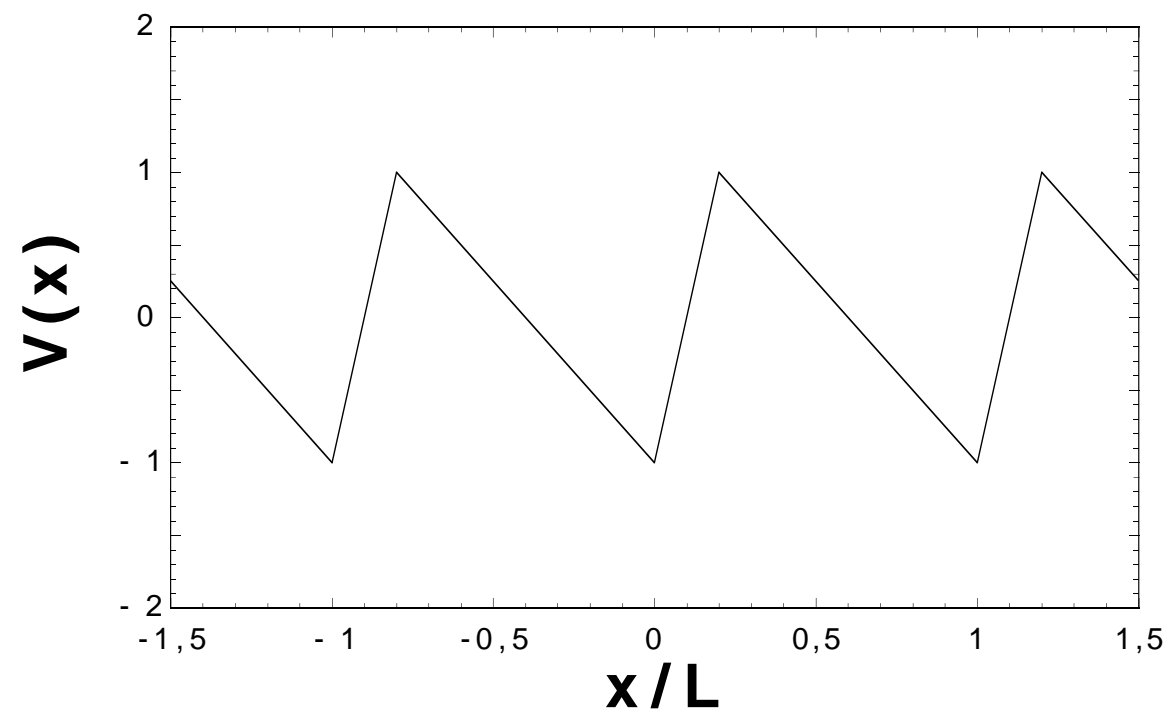

Figure 4.1: Schematic illustration of a piecewise linear "saw-tooth" ratchet potential $V(x)$ (in arbitrary units), consisting of two continuously matched linear pieces per period $L$, one with negative and one with positive slope, but otherwise asymmetric.

For more general potentials $V(x)$ and drivings $f(t)$, things become more complicated. As seen in section 4.1, the current $\langle\dot{x}\rangle$ approaches zero both for very fast and slow switching of $f(t)$ between \pm 1 . As long as the potential $V(x)$ is sufficiently similar to the simple examples from figures 2.2 and 4.1, a single maximum of $\langle\dot{x}\rangle$ develops at some intermediate switching time. For more complicated, suitably chosen potentials $V(x)$, the existence of current inversions follows from section 3.6 and has been exemplified in [271], see also 216, 272]. For a few additional quantitative analytical results we also refer to the subsequent section 4.3 on fluctuating potential ratchets, which includes the on-off scenario as a special case.

\subsubsection{Experimental realizations}

The theoretically predicted pumping effect $\langle\dot{x}\rangle \neq 0$ has been demonstrated experimentally by Rousselet, Salome, Ajdari, and Prost [38] by means of colloidal polysterene latex spheres, suspended in solution and exposed to a dielectric "ratchet"-potential, created by a series of interdigitated "Christmas-tree" electrodes which were deposited on a glass slide by photolithography and which were turned on and off periodically. With one adjustable parameter, accounting for the uncertainty about the shape of the one-dimensional "effective" potential $V(x)$, the agreement of the measured currents $\langle\dot{x}\rangle$ with the theory from [34 turned out to be quite good for all considered particle diameters between $0.2 \mu \mathrm{m}$ and $1 \mu \mathrm{m}$. 
A very similar experimental setup was used by Faucheux and Libchaber 273] (see also [274]) but they went one step further in that they studied solutions containing two different species of particles at a time, and demonstrated experimentally that they can be separated. Again, the quantitative findings depend on the particle size not only via the corresponding viscous friction coefficient $\eta$ but also via slightly different "effective" potentials $V(x)$.

A further experimental verification of the on-off ratchet scheme by Faucheux, Bourdieu, Kaplan, and Libchaber [275] works with single $1.5 \mu \mathrm{m}$ diameter polysterene spheres, confined to an effective one-dimensional "ratchet"-potential by laser-optical trapping methods (optical tweezers). The characteristics of such an optical ratchet potential can be determined with satisfactory accuracy, leading to a quite good quantitative agreement of the the observed ratchet effect with the simple theory from 34 without any adjustable parameters.

A first step towards a practical usable pumping and separation device was achieved by Gorre-Talini, Jeanjean, and Silberzan [276] (see also [277]). Due to their well defined geometry, also in this experiment latex spheres diluted in water with diameters mostly between $0.5 \mu \mathrm{m}$ and $2.5 \mu \mathrm{m}$ were used, but in principle nothing speaks against replacing them e.g. by micrometer sized biological objects like DNA, viruses, or chromosomes. The main ingredient for creating the (electrostatic) "ratchet"-potential is an optical diffraction grating, commercially available in a variety of periods and asymmetries. This setup overcomes many of the practical drawbacks of its above mentioned predecessors, agrees well with the simple theory from [34] without any fitting, and the predicted separating power may well lead to the development of a serious alternative to existing standard particle separation methods.

As regards transport and separation of DNA, all the so far discussed setups are expected to cease working satisfactorily for DNA fragments below one kilobase. By means of an interdigitated electrode array Bader and coworkers 278, 279 successfully micromachined an on-off ratchet on a silicon-chip, capable to transport DNA molecules of 25-100 bases in aqueous solution. With some improvements, such a "lab-on-a-chip" device could indeed provide a feasible alternative to the usual electrophoresis methods for nucleic acid separation, one of the central preprocessing tasks e.g. in the Human Genome Project [278 280].

As pointed out in [277, not only the separation of large DNA molecules by present standard methods seems to have become one of the major bottlenecks in sequencing programs 281, but also chromosomes, viruses, or cells exhibiting major biological differences may only differ very little from the physicochemical viewpoint, thus making their separation highly challenging [282, 283].

\subsection{Fluctuating potential ratchets}

The fluctuating potential ratchet model has been introduced practically simultaneously 1994 by Astumian and Bier [15] and independently by Prost, Chauwin, Peliti, and Ajdari [13]. The model is given by the same type of overdamped dynamics as the on-off ratchet in (4.11) except that the amplitude modulations $f(t)$ are no

\footnotetext{
${ }^{5}$ About 100 metallic strips perpendicular to the transport axis with alternating smaller and larger spacings are either alternatingly charged ("on") or all uncharged ("off").

${ }^{6}$ The closely related work by Peskin, Ermentrout, and Oster 17 came somewhat later and mainly focuses on the special case of an on-off ratchet.
} 
longer restricted to the two values \pm 1 . In other words, the time-dependent potential $V(x)[1+f(t)]$, to which the Brownian particle $x(t)$ is exposed, has always the same shape but its amplitude changes in the course of time between two or more values. Thus the on-off scheme is included as a special case throughout the considerations of this section.

One central and completely general feature of the fluctuating potential ratchet scheme readily follows from (4.11). Namely, within each spatial period there are $x$ values where $V^{\prime}(x)$ is zero. These points cannot be crossed by $x(t)$ in the overdamped dynamics (4.11) without the help of the fluctuations $\xi(t)$. In other words, in an overdamped fluctuating potential ratchet, thermal noise is indispensable for a finite current $\langle\dot{x}\rangle$. A second, completely general conclusion has been pointed out already in section 3.3, namely that only asymmetric potentials $V(x)$ admit a ratchet effect.

For the latter reason, we will mainly concentrate on the simplest non-trivial case of an asymmetric potential $V(x)$ in combination with a symmetric driving $f(t)$. As usual, one option in (4.11) is a periodic driving $f(t)$, see e.g. 271, 284, 285. For asymptotic results for fast periodic driving $f(t)$ we refer to section 6.3. In the following we focus, in accordance with most of the existing literature, on the two particularly simple examples of a stationary random processes $f(t)$ as introduced in section 3.1, see also the asymptotic result (4.10) for general fast stochastically fluctuating potentials.

A well-known experimental phenomenon which can be interpreted by means of a fluctuating potential ratchet scheme is the photoalignement (absorbtion-induced optical reorientation) of nematic liquid crystals [286 289], exemplifying the general considerations in [13]. Another realization by means of a Josephson junction decive similarly to that proposed in [290] (cf. section 5.3.3) is presently planned by the same authors. The large variety of potential applications of the fluctuating potential ratchet mechanism for small-scale pumping devices is the subject of [285].

\subsubsection{Dichotomous potential fluctuations}

The case that the amplitude modulations $f(t)$ in (4.11) are given by a symmetric dichotomous noise (see (3.12), (3.13)) has been considered in 13, 15, 17]. A numerical simulation of the time discretized stochastic dynamics (4.11) similarly as in equation (2.7) is straightforward. Equivalent to this stochastic dynamics is the following master equation for the joint probability densities $P_{ \pm}(x, t)$ that at time $t$ the particle resides at the position $x$ and the dichotomous process $f(t)$ is in the states $\pm \sigma$, respectively:

$$
\begin{aligned}
\frac{\partial}{\partial t} P_{+}(x, t)= & \frac{\partial}{\partial x}\left\{\frac{(1+\sigma) V^{\prime}(x)}{\eta} P_{+}(x, t)\right\}+\frac{k_{B} T}{\eta} \frac{\partial^{2}}{\partial x^{2}} P_{+}(x, t) \\
& -\gamma P_{+}(x, t)+\gamma P_{-}(x, t) \\
\frac{\partial}{\partial t} P_{-}(x, t)= & \frac{\partial}{\partial x}\left\{\frac{(1-\sigma) V^{\prime}(x)}{\eta} P_{-}(x, t)\right\}+\frac{k_{B} T}{\eta} \frac{\partial^{2}}{\partial x^{2}} P_{-}(x, t) \\
& -\gamma P_{-}(x, t)+\gamma P_{+}(x, t) .
\end{aligned}
$$

The derivation of the first two terms on the right hand side of (4.12) and (4.13) is completely analogous to the derivation of the Fokker-Planck equation (2.14): The first terms (drift terms) account for the Liouville-type evolution of the probability densities $P_{ \pm}(x, t)$ under the action of the respective force fields $-V^{\prime}(x)[1 \pm \sigma]$. The second terms (diffusion terms) describe the diffusive broadening of the probability densities 
due to the thermal white noise $\xi(t)$ of strength $2 \eta k_{B} T$ in (4.11). The last two terms in (4.12) and (4.13) are loss and gain terms due to the transitions of $f(t)$ between the two "states" $\pm \sigma$ with transition probability (flip rate) $\gamma$.

For the marginal density $P(x, t):=P_{+}(x, t)+P_{-}(x, t)$ one recovers from (4.12), (4.13) a master equation of the general form (2.17), whence the particle current 2.19) follows. Like in section 2.4 one sees that it suffices to focus on spatial periodic "reduced" distributions $\hat{P}_{ \pm}(x+L, t)=\hat{P}_{ \pm}(x, t)$ in order to calculate the average current $\langle\dot{x}\rangle$ and that in the long time limit $t \rightarrow \infty$ a unique steady state $\hat{P}_{ \pm}^{s t}(x)$ is approached. However, explicit analytical expressions for the probability densities and the particle current in the steady state can only be obtained in special cases. A prominent such case is that of a "saw-tooth" ratchet-potential as depicted in figure 4.1. Within each interval of constant slope $V^{\prime}(x)$, the steady state solutions of (4.12), (4.13) can be readily determined which then have to be matched together. For the straightforward but rather tedious technical details of such a calculation we refer to 15,291. The resulting expression for the current $\langle\dot{x}\rangle$ is awkward and not very illuminating. Qualitatively, the results are like for the on-off scheme (see also figure 2.6): If the local minima of the saw-tooth potential are closer to their neighboring maxima to the right than to the left, then the current is positive for all finite temperatures $T$ and flipping rates $\gamma$ of the dichotomous noise, and (cf. section 3.1) the current approaches zero if $T$ or $\gamma$ tends either to zero or to infinity [13, 15].

As long as the potential is sufficiently similar to a saw-tooth potential such that one can identify essentially one steep and one flat slope per period $L$, qualitatively unchanged results are obtained, and similarly for more general potential fluctuations $f(t)$. In this sense, the "natural" current direction in a pulsating ratchet is given by the sign of the steep potential slope.

For more general potentials $V(x)$, the same qualitative asymptotic features are expected for large and small $T$ and $\gamma$. However, as shown in section 3.6, the current direction may change as a function of any model parameter. So, general hand weaving predictions about the sign are impossible, not to mention quantitative estimates for $\langle\dot{x}\rangle$, as already the small- $\tau$ result (4.10) demonstrates.

A case of particular conceptual interest is the singular perturbation expansion about the unperturbed situation with vanishing thermal noise, $T=0$, and vanishing particle current $\langle\dot{x}\rangle=0$. On condition that the local potential extrema subsist for all values of $f(t)$, i.e. $|\sigma|<1$, the transitions between neighboring minima of the potential will be very rare for small $T$. As a consequence, the current $\langle\dot{x}\rangle$ can be described along the activated barrier crossing approach from section 3.8. Specifically, for our present situation of thermally assisted transitions across barriers with dichotomous amplitude fluctuations, the results of the singular perturbation theory from [254] are immediately applicable.

While for the case of a sawtooth ratchet potential $V(x)$, which is subjected to symmetric dichotomous amplitude fluctuations $f(t)$ in (4.11), current inversions can be ruled out [15], for more complicated fluctuations $f(t)$ this is no longer the case: The occurrence of a current inversion in a sawtooth potential $V(x)$ has been demonstrated for asymmetric (but still unbiased) dichotomous noise [216, 271, 272], for sums of dichotomous processes [266], and for a three-state noise [292]. Originally, the inversion effect has been reported in those works [216, 266, 271, 272, 292] for certain parameters characterizing the noise $f(t)$, but according to section 3.6 the effect immediately carries over to any parameter of the model. 


\subsubsection{Gaussian potential fluctuations}

Next we turn to the second archetypal driving $f(t)$, namely correlated Gaussian noise of the Ornstein-Uhlenbeck type (3.13), (3.15). If both, the thermal noise and the Ornstein-Uhlenbeck noise driven barrier fluctuations are sufficiently weak, transitions between neighboring potential wells of $V(x)$ are rare, and a considerable collection of analytical results 68, 243, 244, 246 248] (see also further references in [68]) - obtained in the context of the "resonant activation" effect - can be immediately applied via (3.55).

\section{Fast fluctuations (multiplicative white noise)}

In section 4.1 we have discussed for a general flashing ratchet scheme the asymptotics for a stochastic $f(t)$ with small correlation time $\tau$ under the assumption that the distribution (3.11) of the noise is kept fixed upon variation of $\tau$ (constant variance scaling). In the special case that the general flashing potential $V(x, f(t))$ in (3.1) depends linearly on its argument $f(t)$, as it is the case in our present fluctuating potential ratchet model (4.11), a second interesting scaling for small $\tau$ is possible: Namely, one keeps the intensity $\int d t\langle f(t) f(0)\rangle$ of the noise constant upon variation of $\tau$. The distinguishing feature of this so-called constant intensity scaling [265] is the emergence of a sensible white noise limit $\tau \rightarrow 0$ in the sense that the effect of the noise $f(t)$ approaches a non-trivial limiting behavior (for constant variance scaling the noise has no effect for $\tau \rightarrow 0$ ). Since the limit depends on the detailed properties of the noise $f(t)$, we focus on the archetypal example of Ornstein-Uhlenbeck noise (3.13), (3.15) with a constant ( $\tau$-independent) intensity

$$
Q:=\sigma^{2} / \tau,
$$

i.e. the variance $\sigma^{2}$ acquires an implicit dependence on $\tau$. In other words, the correlation (3.13) takes the form

$$
\langle f(t) f(s)\rangle=2 Q \delta_{\tau}(t-s)
$$

where

$$
\delta_{\tau}(t):=\frac{1}{2 \tau} e^{-|t| / \tau}
$$

approaches a Dirac-delta function for $\tau \rightarrow 0$.

While this choice of $f(t)$ is clearly of little practical relevance, it gives rise to a model that shares many interesting features with more realistic setups, but, in contrast to them, can be solved in closed analytical form [293]. Furthermore, this exactly solvable model will serve a the basis for our discussion of collective phenomena in section 9.2 . A mathematically similar setup, however, with as starting point a rather different physical system, will also be encountered in section 6.1.

The fact that the coupling strength $V^{\prime}(x(t))$ of the noise $f(t)$ in (4.11) depends on the state $x(t)$ of the system (so-called multiplicative noise) makes the white noise limit $\tau \rightarrow 0$ for constant intensity scaling subtle (so-called Ito-versus-Stratonovich problem 63, 99 101]). The basic reason is [294] that the $\tau \rightarrow 0$ limit does not commute with the $\hat{m} \rightarrow 0$ limit in (2.1) and the $\Delta t \rightarrow 0$ limit in (2.7). We will always assume in the following that the $\hat{m} \rightarrow 0$ and $\Delta t \rightarrow 0$ limits are performed first and that $\tau$ 
becomes small only afterwards. For the sake of completeness we mention that this sequence of limits amounts 294 to treating the multiplicative noise in (4.11) in the so called sense of Stratonovich [63, 99 101], though we will not make use of this fact in the following but rather implicitly derive it.

No such problem arises as far as the (additive) thermal noise $\xi(t)$ in (4.11) is concerned. Hence, we can replace for the moment the Dirac delta in (3.2) by the pre-Dirac-function $\delta_{\tau}(t)$ from (4.16) and postpone the limit $\tau \rightarrow 0$ according to our purposes. It follows that in (4.11) the sum of the two independent, unbiased, $\delta_{\tau^{-}}$ correlated Gaussian noises $-V^{\prime}(x(t)) f(t)$ and $\xi(t)$ are statistically equivalent】 to a single unbiased Gaussian multiplicative noise $\xi_{\tau}(t)$ of the form

$$
\begin{aligned}
& \dot{x}(t)=-\frac{V^{\prime}(x(t))}{\eta}+g(x(t)) \xi_{\tau}(t) \\
& g(x):=\left[\frac{k_{B} T}{\eta}+Q\left(\frac{V^{\prime}(x)}{\eta}\right)^{2}\right]^{1 / 2}
\end{aligned}
$$

with correlation

$$
\left\langle\xi_{\tau}(t) \xi_{\tau}(s)\right\rangle=2 \delta_{\tau}(t-s)
$$

By means of the auxiliary variable

$$
y(x):=\int_{0}^{x} \frac{d \bar{x}}{g(\bar{x})}
$$

it follows from (4.17) that $y(t):=y(x(t))$ satisfies the stochastic dynamics

$$
\dot{y}(t)=-\frac{d}{d y} \phi(x(y(t)))+\xi_{\tau}(t),
$$

where $x(y)$ is the inverse of (4.20) (which obviously exists) and where

$$
\phi(x):=\int_{0}^{x} d \bar{x} \frac{V^{\prime}(\bar{x})}{\eta g^{2}(\bar{x})} .
$$

Next we perform the white noise limit $\tau \rightarrow 0$ in 4.21). The corresponding FokkerPlanck equation for $P(y, t)$ follows by comparison with (2.6) and (2.14), reading

$$
\frac{\partial}{\partial t} P(y, t)=\frac{\partial}{\partial y}\left\{\left[\frac{d}{d y} \phi(x(y))\right] P(y, t)\right\}+\frac{\partial^{2}}{\partial y^{2}} P(y, t) \text {. }
$$

The probability densities $P(x, t)$ and $P(y, t)$ are connected by the obvious relation 101

$$
P(x, t)=\int_{-\infty}^{\infty} d y \delta(x-x(y)) P(y, t)=P(y(x), t) / g(x) .
$$

Upon introducing (4.24) into (4.23) we finally obtain a Fokker-Planck equation (2.17) for $P(x, t)$ with probability current

$$
J(x, t)=-\left\{\frac{V^{\prime}(x)}{\eta}+g(x) \frac{\partial}{\partial x} g(x)\right\} P(x, t) .
$$

\footnotetext{
${ }^{7}$ Proof: The two noises $\xi_{1}(t):=-V^{\prime}(x(t)) f(t)+\xi(t)$ and $\xi_{2}(t):=\sqrt{2 g(x(t))} \xi_{\tau}(t)$ with $g(x)$ from (4.18) and $\xi_{\tau}(t)$ from 4.19 are both Gaussian, have zero mean, and the same correlation, thus all their statistical properties are the same.
} 
Along the same calculations as in section 2.4 one now can derive for the reduced density (2.22) in the steady state the result 112 115] (see also (2.36))

$$
\hat{P}^{s t}(x)=\mathcal{N} \frac{e^{-\phi(x)}}{g(x)} \int_{x}^{x+L} d y \frac{e^{\phi(y)}}{g(y)},
$$

where the normalization $\mathcal{N}$ is fixed through (2.25), and for the particle current 2.26) one finds

$$
\langle\dot{x}\rangle=L \mathcal{N}\left[1-e^{\phi(L)-\phi(0)}\right] .
$$

\section{Discussion}

Our first observation is [293, 295] that the sign of the current is completely determined by the (reversed) sign of $\phi(L)-\phi(0)=\phi(L)$ (note that $\phi(0)=0$ ). Especially, the current vanishes if and only if $\phi(L)=\phi(0)=0$. As expected, this is the case in the absence of the potential fluctuations $(Q=0$, cf. section 2.4) or if $V(x)$ is symmetric. In any other case, we can infer that the current will be typically non-zero, notwithstanding the fact that only white noises are acting.

These basic qualitative conclusions become immediately obvious upon inspection of the transformed dynamics (4.21): The effective potential $\phi(x(y))$ from (4.22) is periodic in $y$ if and only if $\phi(L)=0$. In this case an effective Smoluchowski-Feynman ratchet (2.6) arises with the result $\langle\dot{y}\rangle=0$. If $\phi(L) \neq 0$, we are dealing in (4.21) with a tilted Smoluchowski-Feynman ratchet (cf. section 2.5), yielding a current $\langle\dot{y}\rangle$ with a sign opposite to that of $\phi(L)$. Considering that the ensemble average $\langle\dot{x}\rangle$ is equivalent to the time average of a single realization (3.5) and similarly for $y(t)$, it follows from (4.20) that

$$
\langle\dot{y}\rangle=\frac{\langle\dot{x}\rangle}{L} \int_{0}^{L} \frac{d x}{g(x)}
$$

especially the sign of $\langle\dot{x}\rangle$ must be equal to that of $\langle\dot{y}\rangle$. Remarkably, this exact relation (4.28) between the currents in the original (4.17) and the transformed (4.21) dynamics remains valid for arbitrary (not necessarily Gaussian white) noises $\xi_{\tau}(t)$ 296.

As an example we consider a piecewise linear potential with three continuously matched linear pieces per period $L$ with the following slopes: $V^{\prime}(x)=-1$ for $-2<$ $x<0, V^{\prime}(x)=\lambda$ for $0<x<1$, and $V^{\prime}(x)=2-\lambda$ for $1<x<2$, where $\lambda \in(0,2)$ is a parameter that can be chosen arbitrarily. Hence, the potential $V(x)$ has a minimum at $x=0$ and barriers of equal height 2 at $x= \pm 2$. Outside this 'fundamental cell' of length $L=4$ the potential is periodically continued 8 . One then finds from (4.22) that

$$
\phi(L)=\frac{2 Q\left(1-\lambda^{2}\right)[Q \lambda(2-\lambda)-3 T]}{(T+Q)\left(T+Q \lambda^{2}\right)\left(T+Q(2-\lambda)^{2}\right)} .
$$

As expected, $\phi(L)$ and thus the current (4.27) vanishes for $\lambda=1$, while for any $\lambda \neq 1$ the quantity $\phi(L)$ changes sign at $Q=3 T / \lambda(2-\lambda)$. It follows that the current $\langle\dot{x}\rangle$, considered as a function of either $T$ or $Q$ undergoes a current reversal. Similar reversals as a function of any model parameter follow according to section 3.6.

\footnotetext{
${ }^{8}$ In doing so we are working in dimensionless units (cf. section A.4 in Appendix A) with $\Delta V=2$, $L=4$, and $\eta=k_{B}=1$.
} 
A somewhat similar observation of a current inversion arising upon considering a slight modification of a symmetric sawtooth-potential $V(x)$ has been reported for dichotomous (and other) potential fluctuations in [229], see also [216, 271, 272, 297].

A modified ratchet model dynamics, without any thermal noise and no particular relation between $g(x)$ and $V(x)$ comparable to 4.18), but instead with a Gaussian multiplicative colored noise of finite correlation time $\tau$ in (4.17) has been studies numerically and by means of various approximations in 295,296]. The physical viewpoint in these works is thus closely related to that of the Seebeck ratchet scheme from section 6.1 rather than the fluctuating potential ratchet model of the present section.

\subsection{Traveling potential ratchets}

In this section we consider a special case of the stochastic dynamics (4.1) of the form

$$
\eta \dot{x}(t)=-V^{\prime}(x(t)-f(t))+\xi(t) .
$$

As usual, we are concentrating on the overdamped limit and the thermal fluctuations are modeled by unbiased Gaussian white noise $\xi(t)$ of strength $2 \eta k_{B} T$. Further, $V(x)$ is a periodic, but not necessarily asymmetric potential with period $L$. Thus, the effective potential experienced by the particle in (4.30) is traveling along the $x$ axis according to the function $f(t)$, which may be either of deterministic or stochastic nature.

Upon introducing the auxiliary variable

$$
y(t):=x(t)-f(t)
$$

in $(4.30)$, we obtain

$$
\eta \dot{y}(t)=-V^{\prime}(y(t))-\eta \dot{f}(t)+\xi(t)
$$

whence the average velocity $\langle\dot{x}\rangle$ of the original problem follows as

$$
\langle\dot{x}\rangle=\langle\dot{f}\rangle+\langle\dot{y}\rangle
$$

\subsubsection{Genuine traveling potentials}

As a first example of a so-called genuine traveling potential ratchet we consider a deterministic function $f(t)$ of the form

$$
f(t)=u t
$$

In other words, the potential $V(x-f(t))$ in (4.30) is given by a periodic array of traps (local minima of the potential), traveling at a constant velocity $u$ along the $x$-axis. Hence, (4.30) models basically the working principle of a screw or a screwlike pumping device - both invented by Archimedes [298] - in the presence of random perturbations. Qualitatively, we expect that the Brownian particle $x(t)$ will be dragged into the direction of the traveling potential traps. Next, we note that with (4.34), the auxiliary $y$-dynamics (4.32) describes the well-known overdamped motion in a "tilted 
washboard" potential' [99, 112 115]. Quantitatively, upon comparison of (4.31)-(4.34) with (2.34)-(2.37) the average velocity in the steady state takes the form

$$
\langle\dot{x}\rangle=u-\frac{L k_{B} T\left[e^{\eta u L / k_{B} T}-1\right]}{\eta \int_{0}^{L} d x \int_{x}^{x+L} d y e^{[V(y)-V(x)+(y-x) \eta u] / k_{B} T}} .
$$

Though this formula looks somewhat complicated, one sees that typically $\langle\dot{x}\rangle \neq 0$ with the expected exceptions that either $u=0$ or $V^{\prime}(x) \equiv 0$. Especially, a broken spatial symmetry of the potential $V(x)$ is not necessary for a finite current $\langle\dot{x}\rangle$. Furthermore, thermal noise is not necessary either: For $T \rightarrow 0$ one obtains directly from (4.31), (4.32) that

$$
\langle\dot{x}\rangle= \begin{cases}u-\frac{L}{\int_{0}^{L} \frac{d x}{u+V^{\prime}(x) / \eta}} & \text { if } u+V^{\prime}(x) / \eta \neq 0 \text { for all } x \\ u & \text { otherwise. }\end{cases}
$$

This deterministic behavior already captures the essential features of the more involved finite-T expression (4.35): Namely, $\langle\dot{x}\rangle$ has always the same sign as u but is never larger in modulus, in agreement with what one would have naively expected there are two basic "modes" of motion in (4.36). In the first case in (4.36), i.e. for large speeds $u$, the Brownian particle is only "loosely coupled" to the traveling potential (cf. section 2.7), it behaves like a swimmer afloat on the surface of the ocean and may thus be called a Brownian swimmer 299]. In the second case in (4.36), i.e. for small speeds $u$, we have a Brownian surfer [299], "riding" in a tightly coupled way on the traveling potential in (4.30), 4.34). The current $\langle\dot{x}\rangle$ tends to zero for both very small and very large speeds $u$, and has a maximum at the largest $u$ which still supports the surfing mode.

More general types of genuine traveling potential ratchets are obtained by supplementing the uniformly traveling contribution in (4.34) by an additional unbiased periodic function of $t$ or by a stationary random process. In the transformed dynamics (4.32) this yields a tilting ratchet mechanism with an additional constant external force $-\eta u$ as treated in chapter 5 in more details.

\section{Applications}

Out of the innumerable theoretical and experimental applications of the above described simple pumping scheme (4.34) we can only mention here a small selection.

\footnotetext{
${ }^{9}$ Note that a positive velocity $u$ corresponds to a washboard "tilted to the left".

${ }^{10}$ Particles $x(t)$, moving opposite to the traveling potential in 4.30 ), or, equivalently, particles $y(t)$ sliding down the tilted washboard faster than in the absence of a potential $V(y)$ (faster than $-\dot{f}(t)=$ $-u$ ) would indeed be quite unexpected. Since we did not find a rigorous proof in the literature, we give one herewith: If a function $f(x)$ is concave on an interval $I$, i.e. $f(\lambda x+(1-\lambda) y) \leq \lambda f(x)+(1-\lambda) f(y)$ for all $\lambda \in[0,1]$ and $x, y \in I$, then it follows by induction that $f\left(N^{-1} \sum_{i=1}^{N} X_{i}\right) \leq N^{-1} \sum_{i=1}^{N} f\left(X_{i}\right)$ for all $X_{1, \ldots, N} \in I$. Choosing $u>V^{\prime}(x) / \eta$ for all $x$ (especially $u>0$ ), $f(x)=1 / x, I=[0, \infty]$, and $X_{i}=u+V^{\prime}(i / N)$ it follows for $N \rightarrow \infty$ that $u^{-1}=\left[\int_{0}^{1} d x\left(u+V^{\prime}(x)\right)\right]^{-1} \leq \int_{0}^{1} d x\left(u+V^{\prime}(x)\right)^{-1}$. Working in dimensionless units with $L=\eta=1$ (cf. section A.4 in Appendix A) we can infer that $\langle\dot{x}\rangle \geq 0$ in (4.36) if $u \geq 0$, and similarly $\langle\dot{x}\rangle \leq 0$ if $u \leq 0$. Choosing $f(x)=\exp \{-x\}, I=\mathbf{R}$, $X_{i}=V(i / N)-V(i / N+z)$ it follows that $1=\exp \left\{\int_{0}^{1} d x[V(\bar{x}+z)-V(x)]\right\} \leq \int_{0}^{1} d x \exp \{V(x+z)-V(x)\}$ for any $z$. Hence, $\int_{0}^{1} d x \int_{x}^{x+1} d y \exp \{u(y-x)+V(y)-V(x)\}=\int_{0}^{1} d z \exp \{u z\} \int_{0}^{1} d x \exp \{V(x+z)-$ $V(x)\} \geq \int_{0}^{1} d z \exp \{u z\}=[\exp \{u\}-1] / u$. It follows that $\langle\dot{x}\rangle \geq 0$ in 4.35 if $u \geq 0$, and similarly $\langle\dot{x}\rangle \leq 0$ if $u \leq 0$.

${ }^{11}$ In the corresponding noiseless tilted washboard dynamics $(4.32$, the particle $y(t)$ permanently travels downhill in the first case, while it quickly comes to a halt in the second.
} 
The most obvious one is a particle, either suspended in a fluid or floating on its surface, in the presence of a traveling wave 299 304]. The resulting drift (4.36) has been predicted in the deterministic case $(T=0)$ already by Stokes [305]. In the presence of thermal fluctuations $(T>0)$, the closed analytical solution (4.35) has been pointed out in [301, 303]. The effect of finite inertia has been discussed in [299], based on the known approximative solutions for the corresponding tilted washboard dynamics 99. A model with an asymmetric potential $V(x)$ and a dichotomous instead of a Gaussian white noise $\xi(t)$ in (4.30) has been studied in [304] with the possibility of current inversions as its most remarkable feature, i.e. particles can now even move in the direction opposite to that of the velocity $u$. This result can be readily understood as a consequence of the ratchet effect in the equivalent fluctuating force ratchet 4.32), (4.34), cf. section 5.1.2. Further generalizations for superpositions of traveling waves in arbitrary dimensions are due to [300, 306], which will be discussed in somewhat more detail in section 4.5.1.

The mesoscopic analog of Archimedes' water pump is the adiabatic quantum electron pump by Thouless [307]. This theoretical concept has been realized in a quantum dot experiment in [308], triggering in turn further theoretical studies [309 311]. Similar single electron pumps, however, operating in the classical regime [308], have been realized in 312 315. For additional closely related single electron pumping experiments, see also 316, 317.

A theoretical analysis of diffusion (unpredictability) in clocked reversible computers in terms of a Brownian motion in a traveling potential is given in 318.

Experimental studies of Brownian particles $(2 \mu \mathrm{m}$ diameter polysterene spheres in water), moving on a circle in the presence of a traveling optical trap, have been reported in 319] and are in good agreement with the simple theoretical model 4.30), (4.34).

Single-electron transport by high-frequency surface acoustic waves in a semiconductor heterostructure device has been demonstrated for example in [320]. A more sophisticated variant with excitons (electron-hole pairs) instead of electrons, which is thus able to transport "light", has been realized in [321].

Though an asymmetry in the periodic potential $V(x)$ is not necessary to produce a current in (4.30), (4.34), one may of course consider traveling ratchet-shaped potentials nevertheless. This situation has been addressed for instance in 322, 323, leading to interesting effects for traveling wave trains of finite spatial extension (i.e. $V^{\prime}(x) \rightarrow 0$ for $x \rightarrow \pm \infty$ ) which are reflected at a wall and then pass by the same particle again in the opposite direction 299].

One basic effect of pumping particles by a traveling potential is a concentration gradient. The inversions, namely making a potential travel by exploiting a particle flux (e.g. due to a concentration gradient) is also possible [324], as exemplified by the chiral dynamics of a "molecular wind-mill" 325. Note that useful (mechanical) work can be gained in either way.

A number of further applications in plasma physics and quantum optics have been compiled in 299. In fact, a great variety of engines are operating in a cyclic manner with a broken symmetry between looping forward and backward and may thus be classified as ratchet systems, typically of the traveling potential type. The examples of screws, water pumps, propellers, or equally spaced traveling cars (representing the traveling potential minima for the passengers) demonstrate a certain danger of invoking an overwhelming practical relevance while the underlying basic principle may become 
trivial from the conceptual viewpoint of contemporary theoretical physics. Furthermore, these largely mechanical examples together with our above findings that neither a broken symmetry nor thermal noise are necessary, current inversions are impossible, and the tight $x(t)$-to- $f(t)$-coupling (see section 2.7) in the most important case of small speeds $u$ show that many characteristic aspects of the Brownian motor concept are actually absent in genuine traveling potential ratchets.

While the general framework in section 3.1 has been purposefully set such as to include traveling potential ratchets, they are at the boarder line in so far as they often involve a quite obvious a priori preferential direction of transport. The boarder between the realm of Brownian motors and that of "pumping between reservoirs" is definitely crossed when in addition the spatial periodicity and noise effects play no longer a central role.

\subsubsection{Improper traveling potentials}

Next we turn to the simples type of a so-called improper traveling potential ratchet, arising through a modification of the driving function $f(t)$ from (4.34) of the form

$$
f(t)=u t-\int_{0}^{t} d t^{\prime} \sum_{i=-\infty}^{\infty} n_{i} L \delta\left(t^{\prime}-\tau_{i}\right)
$$

where, the coefficients $n_{i}$ are either deterministically fixed or randomly sampled integers (not necessarily positive). Thus, most of the time the function $f(t)$ changes at a constant rate $u$, but at the special instants $\tau_{i}$ it jumps by an integer multiple of the spatial period $L$. These times are assumed to be ordered, $\tau_{i+1}>\tau_{i}$, and may be either regularly or randomly spaced. The main idea is to choose them such that $f(t)$ in (4.37) becomes an unbiased periodic function of time or a stochastic process with zero average (hence the name "improper traveling potential"). In other words, extending the meaning of the symbol $\langle\dot{f}\rangle$ analogously as in (3.5) we require that

$$
\langle\dot{f}\rangle=\lim _{t \rightarrow \infty} \frac{f(t)}{t}=0
$$

so that $\langle\dot{x}\rangle=\langle\dot{y}\rangle$ in (4.33), i.e. on the long term the discontinuous jumps in (4.37) have to counterbalance the continuous change $u t$. This condition is satisfied if and only if

$$
\mathcal{T} u \bar{n}=L,
$$

where we have introduced

$$
\begin{aligned}
& \bar{n}:=\lim _{k \rightarrow \infty} \frac{1}{2 k+1} \sum_{i=-k}^{k} n_{i}, \\
& \mathcal{T}:=\lim _{k \rightarrow \infty} \frac{1}{2 k+1} \sum_{i=-k}^{k}\left(\tau_{i+1}-\tau_{i}\right) .
\end{aligned}
$$

Especially, the so defined limits (4.40), (4.41) are assumed to exist and to be independent of the considered realization in the case that the summands are randomly sampled. 
Regarding examples, the simples choice of the coefficients $n_{i}$ in (4.37) is $n_{i}=1$ for all $i$. On the other hand, the simplest choice of the times $\tau_{i}$ arises if they are regularly spaced. Then $\mathcal{T}$ in (4.41) is obviously equal to this spacing, i.e.,

$$
\tau_{i}=i \mathcal{T}+\text { const }
$$

Another simple option are random $\tau_{i}$ with a Poisson statistics, i.e. the probability to have $m$ time points in a time interval of duration $t \geq 0$ is

$$
P_{m}(t)=\frac{(t / \mathcal{T})^{m}}{m !} e^{-t / \mathcal{T}}
$$

Then, in accordance with (4.41), $\mathcal{T}$ is again the mean value of $\tau_{i+1}-\tau_{i}$.

Returning to general processes (4.37) with integers $n_{i}$ respecting (4.38) or equivalently (4.39), we now come to the central point of this section, consisting in the following very simple observation: Since the discontinuous jumps of the driving $f(t)$ in (4.37) are always integer multiples of the period $L$ and the potential $V(x)$ is $L$-periodic, the jumps of $f(t)$ in (4.37) do not have any effect whatsoever on the stochastic dynamics (4.30)! In other words, the genuine traveling potential ratchet (4.30), (4.34) is equivalent to the improper traveling potential ratchet (4.30), (4.37), (4.38). Especially, the results (4.35), (4.36) for the current $\langle\dot{x}\rangle$ can be taken over unchanged. Due to (4.33), (4.38) the same results are moreover valid for $\langle\dot{y}\rangle$. With 4.37) the term $\dot{f}(t)$ in the $y$-dynamics (4.32) takes the form

$$
\dot{f}(t)=u-\sum_{i=-\infty}^{\infty} n_{i} L \delta\left(t-\tau_{i}\right) .
$$

In other words, we have found that the dynamics (4.32), (4.44) with (4.38) or equivalently (4.39), admits a closed analytic solution. In the special case of a Poisson statistics (4.43) the random process (4.44) is called a Poissonian shot noise 178, 326 329. Its mean value is zero owing to (4.38), and its correlation is found to be

$$
\begin{aligned}
& \langle\dot{f}(t) \dot{f}(s)\rangle=\frac{\overline{n^{2}} L^{2}}{\mathcal{T}} \delta(t-s) \\
& \overline{n^{2}}:=\lim _{k \rightarrow \infty} \frac{1}{2 k+1} \sum_{i=-k}^{k} n_{i}^{2},
\end{aligned}
$$

i.e. the shot noise is uncorrelated in time (white noise). The conclusion that a stochastic dynamics (4.32) in the presence of a white shot noise 330 333 or an unbiased periodic driving of the form (4.44) (with $n_{i}$ being integers) and simultaneously a white Gaussian noise $\xi(t)$ is equivalent to the Brownian motion in a traveling potential or in a tilted washboard potential and thus exactly solvable is to our knowledge new.

\section{Generalizations, equivalences, applications}

Generalizations of the above arguments are obvious and we only mention here a few of them. First, an arbitrary periodic $f(t)$ in (4.30) is equivalent to a dynamics 4.32) with a homogeneous periodic driving force $\dot{f}(t)$. Due to the periodicity of $f(t)$, this force is unbiased in the sense of $(4.38)$ and due to (4.33) the currents of the original (4.30) and the transformed dynamics (4.32) are thus strictly equal. Such a dynamics 
(4.32) will be considered under the labels "rocking ratchets" and "asymmetrically tilting ratchets" in chapter 5 . Both these classes of ratchets are thus equivalent to a Brownian motion in a back-and-forth traveling periodic potential. As we will see, a finite current $\langle\dot{x}\rangle=\langle\dot{y}\rangle \neq 0$ generically occurs if $V(x)$ and/or $\dot{f}(t)$ is asymmetric (see section 3.2) and unless both of them are supersymmetric according to section 3.5. An inversion of the current upon variation of an arbitrary parameter of the model can be designed along the same line of reasoning as in section 3.6.

The above exemplified procedure of transforming a genuine into an improper traveling potential ratchet is obviously very general. Due to (4.38) this transformed model can then be mapped once more onto an unbiased tilting ratchet scheme (4.32). In short, genuine and improper traveling potential models are equivalent to each other and moreover equivalent to a tilting ratchet.

Finally, we turn to a modification of the (genuine) uniformly traveling potential (4.30), (4.34), namely the case that $f(t)$ and thus the periodic potential advance in discrete steps:

$$
f(t)=\int_{0}^{t} d t^{\prime} \sum_{i=-\infty}^{\infty} \Delta_{i} \delta\left(t^{\prime}-\tau_{i}\right) .
$$

As seen before, steps $\Delta_{i}=n_{i} L$ do not have any effect on the dynamics (4.30). Thus, the simplest nontrivial case arises when two subsequent steps add up to one period $L$ :

$$
\Delta_{2 i}=i L, \quad \Delta_{2 i+1}=i L+\lambda, \quad \lambda \in(0, L) .
$$

We furthermore assume that the jumping times $\tau_{i}$ are regularly spaced

$$
\tau_{2 i}=i \mathcal{T}, \quad \tau_{2 i+1}=i \mathcal{T}+\tau, \quad \tau \in(0, \mathcal{T})
$$

For $\lambda=L / 2$ and $\tau=\mathcal{T} / 2$ the signal $f(t)$ in (4.47) is thus a discretized version of (4.34) advancing at equidistant steps in time and in space with the same average speed $u=L / \mathcal{T}$. For other values of $\lambda$ and $\tau$, every second step is modified. More steps per period, random instead of deterministic times $\tau_{i}$ and many other generalizations are possible but do not lead to essential new effects.

Recalling that jumps of $f(t)$ by multiples of $L$ do not affect the dynamics (4.30), we can infer that (4.47)-(4.49) is equivalent to

$$
\begin{aligned}
& f(t)=\left\{\begin{array}{lll}
0 & \text { if } & t \in[0, \tau) \\
\lambda & \text { if } & t \in[\tau, \mathcal{T})
\end{array}\right. \\
& f(t+\mathcal{T})=f(t)
\end{aligned}
$$

i.e., $f(t)$ periodically jumps between the two values 0 and $\lambda$. Such a genuine traveling potential advancing in discrete steps is thus equivalent to a periodic switching between two shifted potentials. The periodicity of $f(t)$ in (4.50) implies (4.38), thus the current in (4.33) agrees with that of the transformed dynamics (4.32), featuring an unbiased additive force $\dot{f}(t)$ with $\delta$-peaks of weight $\lambda$ at $t=i \mathcal{T}$ and weight $-\lambda$ at $t=i \mathcal{T}+\tau$. As before, this equivalent dynamics establishes the connection of a stepwise traveling potential with the rocking and asymmetrically tilting ratchet schemes from chapter 5 .

Without going into the details of the proofs we remark that: (i) A symmetric potential $V(x)$ in combination with $\tau=\mathcal{T} / 2$ in 4.49 implies $\langle\dot{x}\rangle=0$ for any $\lambda$ in 
(4.48). (ii) A symmetric potential $V(x)$ at temperature $T=0$ implies $\langle\dot{x}\rangle=0$ for any $\tau$ and $\lambda$. (iii) For $T>0, \lambda \neq L / 2, \tau \neq \mathcal{T} / 2$ a current $\langle\dot{x}\rangle \neq 0$ is generically expected [214]. (iv) If $V(x)$ is asymmetric 440, 334] or $f(t)$ supports more than two effectively different discrete states (cf. (4.50)), i.e. when transitions between more than two shifted potentials are possible [335, 336], then a ratchet effect $\langle\dot{x}\rangle \neq 0$ is expected generically.

Apart from those peculiarities, one expects basically the same qualitative behavior as for the uniformly traveling potential case (4.34). Quantitative results, confirming this expectation, are exemplified in [40,336. Like in the continuously traveling potential case, there are again two basic modes of motion (cf. the discussion below (4.36)): One which is "loosely" coupled to the traveling potential (Brownian swimmer), and one in which the particle "rides" on the traveling wave (Brownian surfer). This clear cut distinction is washed out by the thermal noise. The detailed dependence of $\langle\dot{x}\rangle$ on model parameters like $\lambda, \tau$, or $\eta$ shows furthermore certain traces [40] of the discrete jumps in (4.47) which are not present in the continuously traveling counterpart (4.34).

An experimental realization of directed motion by switching between two shifted ratchet shaped potentials has been reported in [337. The moving particle is a mercury droplet of about $1 \mathrm{~mm}$ in diameter and the two shifted ratchet potentials are created by suitably positioned electrodes. Both, for periodic and stochastic switching between the two ratchet potentials, the measured displacements agree very well with the simple $T=0$ theory from 40 .

The same ratchet scheme has been implemented experimentally for $\mu m$-sized latex spheres in 277]. The setup is similar to the one by Rousselet, Salome, Ajdari, and Prost [38] and by Faucheux and Libchaber [273] described in section 4.2.1 and thus the same uncertainties when comparing measurements with theory arise. The main difference in [277] is that now two superimposed "Christmas-tree electrodes" are used, shifted against each other so as to generate the two shifted ratchet potentials by switching the applied voltage. A further difference with the on-off experiments [38, 273] is that in such a traveling potential-type setup [277] thermal fluctuations are negligible in very good approximation (cf. section 4.4.1). For two different species of highly diluted particles (latex spheres with $0.2 \mu \mathrm{m}$ and $0.5 \mu \mathrm{m}$ diameters) the theoretically predicted effect that for a suitable choice of parameters, only one of them appreciably moves, was qualitatively verified in the experiment 277.

\subsection{Hybrids and further generalizations}

\subsubsection{Superpositions of traveling potentials}

In this section we consider combinations and other extensions of the fluctuating potential and traveling potential ratchet schemes from (4.11) and (4.30). As a first example, we consider a pulsating potential ratchet dynamics (4.1) involving superpositions of several traveling potentials 300,306

$$
\begin{aligned}
V(x, f(t)) & =\sum_{i} V_{i}\left(x-u_{i} t\right) \\
V_{i}\left(x+L_{i}\right) & =V_{i}(x) .
\end{aligned}
$$

At variance with all other cases considered in this chapter, the potential (4.51) is thus not periodic in the spatial variable $x$ unless the periods $L_{i}$ are all commensurable with 
each other.

The starting point for an approximate treatment is an expansion of the singlepotential result (4.35) up to the first nontrivial order in $V(x) / k_{B} T$, which turns out to be the second order [300, 301]. The next salient point is that for several potentials one simply can, within the same approximation, add up the contributions from all the single potentials provided that their traveling speeds $u_{i}$ and periods $L_{i}$ are, in modulus, different from each other. In other words, up to second order, no mixed contributions in the amplitudes of the traveling potentials appear [300, 306]. Basically, the reason is that the mismatch of the different temporal and spatial periods only leads to oscillating mixed terms which average out to zero in the long run. Proceeding along this line of reasoning, the final result for the net current $\langle\dot{x}\rangle$ is

$$
\begin{aligned}
\langle\dot{x}\rangle & =\sum_{i} u_{i}\left[\int_{0}^{L_{i}} \frac{d x}{L_{i}} \frac{V_{i}^{2}(x)}{\left(k_{B} T\right)^{2}}-\frac{\alpha_{i}}{e^{\alpha_{i}}-1} \int_{0}^{L_{i}} \frac{d x}{L_{i}} \int_{x}^{x+L_{i}} \frac{d y}{L_{i}} \frac{V_{i}(x) V_{i}(y)}{\left(k_{B} T\right)^{2}} e^{\alpha_{i}(y-x) / L_{i}}\right] \\
\alpha_{i} & :=\eta u_{i} L_{i} / k_{B} T
\end{aligned}
$$

where it is assumed that both terms in the square brackets are small in comparison to unity, i.e., $V_{i}(x) / k_{B} T$ needs to be small, but also $\alpha_{i}$ should be not too large in modulus. Specifically, for sinusoidal traveling potentials of the general form

$$
V(x, f(t))=\sum_{i} A_{i} \sin \left(\frac{2 \pi}{L_{i}}\left(x-u_{i} t\right)+\phi_{i}\right)
$$

one obtains

$$
\langle\dot{x}\rangle=\frac{1}{2\left(k_{B} T\right)^{2}} \sum_{i} u_{i} A_{i}^{2}\left[1+\left(\frac{L_{i} u_{i} \eta}{2 \pi k_{B} T}\right)^{2}\right]^{-1} .
$$

Thus, already with two superimposed potentials with opposite speeds $u_{1}$ and $u_{2}$ and $\left|u_{1}\right| \neq\left|u_{2}\right|,\left|L_{1}\right| \neq\left|L_{2}\right|$ one can tailor the two amplitudes $A_{1}$ and $A_{2}$ such that the current (4.55) will change its direction either upon variation of the temperature $T$ or, at a fixed but finite $T$, upon variation of the friction coefficient $\eta$. While for transport based on a single traveling potential, thermal fluctuations are not important (cf. section 4.4.1), they are thus indispensable for this type of particle separation scheme 300.

There is no reason to expect that the above effect is restricted to potentials of small amplitudes, but beyond this regime quantitative analytical progress becomes cumbersome. Qualitatively, the following very simple prediction is worth mentioning: We consider in (4.1) a potential that is given as a linear combination of two potentials, moving uniformly in opposite directions, i.e., $f(t)=u t$ and

$$
V_{\lambda}(x, f(t)):=\lambda V_{1}(x+u t)+(1-\lambda) V_{0}(x-u t),
$$

where $\lambda$ is a control parameter and the spatial periods of $V_{0}(x)$ and $V_{1}(x)$ may or may not agree. Similarly as in section 3.6 one sees that for a "generic" choice of $V_{0}(x)$ and $V_{1}(x)$ (no "accidental symmetries" of $V_{\lambda}(x, f(t))$ for any $\left.\lambda \in(0,1)\right)$ a $\lambda$-value must exists at which the current $\langle\dot{x}\rangle$ exhibits an inversion upon variation of an arbitrarily chosen parameter of the model (4.1). Note that in contrast to the prediction from (4.53) the present conclusion holds even if the thermal noise $\xi(t)$ in (4.1) is zero, see equation (4.36). 
Another variation with one static and one traveling potential, i.e.

$$
V(x, f(t)):=V_{0}(x)+V_{1}(x-u t),
$$

has been analyzed in 338] in the zero temperature limit $\xi(t) \equiv 0$ in (4.1). If at least one of the two potentials $V_{0}(x), V_{1}(x)$ is asymmetric and their relative amplitudes are properly chosen then the traveling potential is able to drag the particle $x(t)$ in (4.1) into one direction. However, if the traveling direction is reversed $(u \mapsto-u)$, the particle cannot be dragged in that direction anymore due to the asymmetry of the potential. The possibility of such a behavior becomes particularly obvious in the case of small speeds $u$ such that the particle tends to follow one of the instantaneous local minima of the total potential $V(x, f(t))$ in (4.57): For a very small amplitudes of $V_{1}(x)$, the particle clearly cannot be dragged into either direction, while for very large amplitudes it can be dragged into both directions. Thus there must be an intermediate amplitude of transition from localized to commoving behavior. Due to the spatial asymmetry, this transition amplitude is typically not the same for positive and negative speeds and commoving behavior in only one direction is recovered.

\subsubsection{Generalized pulasting ratchets and experimental realizations}

In the remainder of this section we focus again on periodic potentials (3.3) in the genuine pulsating potential ratchet setting (4.1). Still, the various possibilities of how to choose $V(x, f(t))$ obviously rule out an exhaustive discussion. We will restrict ourselves to a few representative examples which cover most of the existing experimental and theoretical literature and which already exhibit all main features that one may possibly expect in more general cases.

The simplest example is a hybrid of a uniformly traveling and simultaneously fluctuating potential ratchet of the form

$$
V(x, f(t))=V(x-u t)[1+\tilde{f}(t)]
$$

where $f(t):=u t$ and the auxiliary function $\tilde{f}(t):=\tilde{f}(f(t) / u)$ is assumed to be a periodic function of its argument. By means of the same transformation of variables as in (4.31)-(4.33) one can map this model onto a purely fluctuating potential ratchet with a superimposed tilt. Thus a finite current $\langle\dot{x}\rangle$ is generic and the possibility of current inversions is also immediately obvious.

A similar hybrid of a traveling and simultaneously fluctuating potential ratchet arises if $f(t)$ is not given by $u t$ in (4.58) but instead increases in discrete steps like in (4.47). In the simplest case, a model which switches either regularly or randomly between two different potentials $V_{m}(x)(m=1,2)$ arises (cf. equation (4.50), which both have the same shape but are shifted against each other and moreover differ in their amplitudes. Observing that the on-off ratchet is a special case and exhibits current inversions for suitably tailored potentials [40] (cf. section 4.2), the same property follows for the present more general situation.

Going just one step further, one may consider in (4.1) the case of a periodic or random switching between two potentials $V_{m}(x)(m=1,2)$ which have the same spatial period $L$ but are otherwise completely independent of each other. The generic occurrence of a non-vanishing current $\langle\dot{x}\rangle$ and the existence of current inversions for suitably chosen potentials is obvious. 
An experimental study of such a system has been performed by MenneratRobilliard, Lucas, Guibal, Tabosa, Jurczak, Courtois, and Grynberg [339]. Laser cooled Rubidium atoms in the presence of two suitably chosen counterpropagating electromagnetic waves can switch between two effective optical potentials $V_{m}(x)$ with the above described properties. The switching is caused by absorption-spontaneous emission cycles of the Rubidium atoms and results in an average velocity $\langle\dot{x}\rangle$ of the atoms of about $0.1 \mathrm{~m} / \mathrm{s}$. While the simple stochastic dynamics (4.1) with two alternating potentials $V_{m}(x)$ is sufficient for a qualitative explanation of the observed results, a quantitative comparison would require a semiclassical or even full quantum mechanical treatment (see also section 8.4).

Another generalization are so-called asynchronously pulsating ratchets [197] (see also sections 3.4 .2 and 6.7 and especially the so-called sluice-ratchet scheme 198 , 199, 201. In this latter case, the amplitudes of every second potential barrier are periodically oscillating in perfect synchrony, similarly as for a fluctuating potential ratchet. The rest of the potential barriers are also synchronously oscillating in the same way, but with a time-delay of $\mathcal{T} / 4$ (where $\mathcal{T}$ is the time-period). Thus, the Brownian particle $x(t)$ moves forward somewhat similar to a ship in an array of sluices and may achieve $100 \%$ efficiency in the adiabatic limit $\mathcal{T} \rightarrow \infty$ (see section 6.9). An experimental realization by semiconductor superlattice heterostructures is due to [200, 202].

We close with three promising experimental implementations of a pulsating ratchet scheme on a molecular scale which have so far been partially realized. The first one is based on the single triptycene[4] helicene molecules which we already encountered in section 2.1. By means of certain chemical processes which basically play the role of the non-thermal potential fluctuations in the pulsating ratchet scheme, Kelly, De Silva, and Silva 340 342] achieved a unidirectional intramolecular rotary motion. The system is so far only a "partial" Brownian motor in that only rotations by $120^{\circ}$ have been realized.

Monodirectional rotation in a helical alcene molecule with a definite chirality (broken symmetry) has been investigated by Koumura, Zijistra, van Delden, Harada, and Feringa 341, 343]. Basically, ultraviolet radiation induces transitions between two ratchet-shaped potentials which are identical except that they are shifted by half a period. In other words, a photochemical two-state pulsating (or traveling) potential ratchet scheme as anticipated theoretically in [13,28] is realized. Chemically, the lightinduced switching between the two potentials corresponds to a cis-trans isomerization, and each such transition is followed by a thermally activated relaxation process. While experimentally, each of the partial steps of a full cycle has so far been only demonstrated separately, there seems no reason why the system should not be able to also rotate continuously.

Finally, Gimzewski, Joachim and coworkers [344, 345] have visualized single propeller-shaped molecular rotors (hexa-tert-butyl decacyclene) deposited on a $\mathrm{Cu}$ surface by means of scanning tunneling microscopy (STM). Under appropriate conditions, the molecule is observed to perform a thermally driven rotary Brownian motion within an environment which gives rise to a highly asymmetric, ratchet-shaped effective potential of interaction with the rotor. In principle, we are thus dealing with another molecular realization of a Smoluchowski-Feynman ratchet and pawl gadget (cf. section 2.1), but in the present case the time resolution of the STM was too low to confirm the absence of a preferential direction of rotation. As the authors in 344,345 propose, 
with the help of a second non-thermal source of noise, for example a tunnel current, it should be possible to realize a ratchet effect in terms of a preferential direction of rotation. Considering that such a tunnel current would not directly interact with the angular state variable of the system but rather with some internal degree of freedom (of the environment), a pulsating ratchet scheme is expected according to our general analysis from section 3.4 .2 .

\subsection{Biological applications: molecular pumps and motors}

Consider an isothermal chemical reaction in the presence of a catalyst protein, i.e. an enzyme. In the simplest case, the reaction can be described by a single reaction coordinate, cycling through a number of chemical states. A suitable working model is then an overdamped Brownian particle (reaction coordinate) in the presence of thermal fluctuations in a periodic potential. The local minima within one period represent the chemical states and looping once through the chemical cycle in one or the other direction is monitored by a forward or backward displacement of the reaction coordinate by one spatial period. Completing a cycle in one direction means that one entity of reactant molecules have been catalyzed into product molecules, while a cycle in the other direction corresponds to the reverse chemical reaction.

Since our so simplified model is nothing else than a Smoluchowski-Feynman ratchet (2.6), the absence of a net current signals that we are dealing with a chemical process at equilibrium, i.e. the concentrations of reactants and products are at their equilibrium values and are not changing on the average under the action of the enzyme.

If the concentrations of the reactants and products are away from their equilibrium (detailed balance) ratio, then the catalyst molecule will loop through the chemical reaction cycle preferably in one way, namely such that the reaction proceeds towards equilibrium. In the corresponding Smoluchowski-Feynman ratchet model, the periodic potential has to be supplemented by a constant tilt ए2, resulting in a stochastic dynamics of the form (2.34). Note that while the environment of the catalyst is out of equilibrium as far as the concentrations of reactants and products are concerned, the properties of the random environmental noise and of the dissipation mechanism in (2.34) are still the same as for the equilibrium system (2.6).

Usually, one or several transitions between chemical states may also be (in a probabilistic or deterministic sense) accompanied by a change of the geometrical shape (mechanical configuration) of the catalyst molecule ("mechanochemical coupling"). Transitions between such configurations may then be exploited for doing mechanical work. Due to the preferential direction in which these transitions are repeated as time goes on, one can systematically accumulate useful mechanical energy out of the chemical energy by keeping up the nonequilibrium concentrations of reactants and products. This conversion of chemical into mechanical energy reminds one of the working of a macroscopic combustion engine, except that everything is taking place on a molecular scale and thus thermal fluctuations must be added to the picture.

\footnotetext{
${ }^{12}$ For a proper description of an out of equilibrium catalytic cycle on a more sophisticated level, see section 7.3.2. This description is in terms of a discrete chemical state variable $m$. On the same level, a consistent description in terms of a continuous state variable $x$ does not seem to exist (see also [186]) unless it is basically equivalent to the discrete description (activated barrier crossing limit, see section 3.8.
} 
Similarly as for the chemical reaction coordinate, in the simplest case the changes of the geometrical configuration can be described by a single mechanical coordinate, originally living on a circle but easily convertible to a periodic description on the real axis. In the absence of chemical reactions, another Smoluchowski-Feynman ratchet dynamics (2.6) for the mechanical state variable arises. One suggestive way to include the effect of the chemical reaction is the traveling potential scheme (4.30), where $x(t)$ and $f(t)$ are the mechanical and chemical state variables, respectively. Thus, the traveling potential proceeds in a preferential direction in accord with the chemical reaction and thereby is dragging the mechanical coordinate along the same preferential direction [3]. Another possibility is that, instead of producing a traveling potential, the chemical process gives rise to a fluctuating potential to which the mechanical coordinate is exposed, or an even more general type of pulsating periodic potential (4.1).

This general scheme seems to be indeed exploited by nature for numerous intracellular transport processes [23,24]. An example are "molecular pumps" (enzymes) in biological membranes, which transfer ions or small molecules from one side of the membrane to the other by catalyzing ATP (adenosine triphosphate) into ADP (adenosine diphosphate) and $\mathrm{P}_{i}$ (inorganic phosphate) [187, 346]. Another example, also fueled by ATP, are "molecular motors" which are able to travel along intracellular polymer filaments. A detailed discussion of the latter example will be presented in chapter 7 , especially in the final section 7.7. Finally, we remark that in principle nothing speaks against the possibility that the general scheme could be realized not only for enzymes (proteins) but also for much simpler kinds of catalysts.

\subsubsection{Externally driven molecular pumps}

Molecular pumps are enzymes that use energy from ATP hydrolysis to create and maintain concentration gradients of ions or other small molecules like sugars (glucose) or amino acids across membranes. As discussed before, such a chemical process requires that the concentrations of reactants and products are kept away from their equilibrium ratio. In living cells, this task is accomplished by intracellular "energy factories", maintaining the concentration of ATP about 6 decades above its thermal equilibrium value.

Experimentally, there exists another interesting option [16, 187], namely to suppress ATP hydrolysis either by low temperature or by bringing the ATP concentration close to its equilibrium value and instead apply an external time-dependent electric field. Without the field and in the absence of ATP hydrolysis, we thus recover the Smoluchowski-Feynman model (2.6) for the mechanical state variable $x(t)$ of the molecular pump 팩.

Since ATP hydrolysis is suppressed, the chemical state variable, previously denoted by $f(t)$, can be omitted in the following discussion and the same symbol $f(t)$ is now used for the external field. We first consider the case that the field $f(t)$ only couples to the mechanical coordinate $x(t)$ of the enzyme but not to the pumped molecule (e.g. ions are excluded if $f(t)$ is an electrical field). As a consequence, the effective potential $V(x, f(t))$ experienced by the mechanical state variable $x(t)$ changes its shape as

\footnotetext{
${ }^{13}$ Properly speaking, there is also a back-reaction of the mechanical on the chemical state variable. A more detailed discussion of the present modeling framework is given in chapter 7 .

${ }^{14}$ We recall that the mechanical coordinate represents the geometrical shape of the enzyme. Since ions or molecules are mechanically transferred through the membrane, a displacement of the mechanical coordinate monitors at the same time the pumping of ions or molecules.
} 
a function of $f(t)$ but will maintain always the same spatial periodicity. The corresponding model dynamics is thus of the general form (4.1). Though the detailed shape of the fluctuating periodic potential $V(x, f(t))$ is usually not known, the occurrence of a ratchet effect is generically expected for a very broad class of periodic or randomly fluctuating external driving signals $f(t)$. In other words, the molecular pump starts to loop in one or the other preferential direction and so pumps molecules across the membrane. An external driving can thus substitute for the chemical energy from ATP hydrolysis to power the molecular pump, i.e., $f(t)$ in (4.1) may represent either the chemical reaction coordinate or the external driving field, the main consequence $\langle\dot{x}\rangle \neq 0$ is the same.

The more general case that the external field not only induces a pumping of molecules across the membrane but also leads to a production of ATP out of ADP and $\mathrm{P}_{i}$ is discussed in 21,22]. Finally, ATP-driven pumping may also induce electrical fields in the vicinity of the enzyme [21, 22].

If the substance transported by the molecular pump itself couples to the external field $f(t)$, e.g. a ion when $f(t)$ is an electrical field, then the total potential experienced by the mechanical state variable $x(t)$ acquires a tilt in addition to the periodic contribution. If the mechanical coordinate $x(t)$ does not couple to the field, then the periodic contribution to the total potential is always the same and we recover the tilting ratchet scheme from chapter 5 . If the field affects both the transported substance and the enzyme, a combined pulsating and tilting ratchet mechanism will result.

For periodic fields $f(t)$, this effect has been discovered experimentally in 19, 20, 188 and explained theoretically in [21, 22, 347, 348] by means of a model with a discretized mechanical coordinate, hopping between four states at certain rates which change under the influence of the field $f(t)$. Employing the same type of models, the possibility that also a randomly fluctuating field $f(t)$ of zero average may put molecular pumps to work was first predicted in [158, 255] and subsequently verified experimentally in [256, 257. Though these and later discussions 349 351] are conducted mainly in the language of discrete state kinetic models, the underlying physical picture is equivalent to the spatially continuous ratchet paradigm [16, 187, 297, 352, 353]. Indeed, a plot reminiscent of a fluctuating potential ratchet (restricted to a fraction of the full spatial period) appears already in [255] and a full-fledged traveling ratchet scheme is depicted in [354]. Note also the close connection to the resonant activation effect from section 3.8 . 


\section{Chapter 5}

\section{Tilting ratchets}

\subsubsection{Model}

At the focus of this chapter is the one-dimensional overdamped stochastic dynamics

$$
\eta \dot{x}(t)=-V^{\prime}(x(t))+y(t)+\xi(t) .
$$

Here, as discussed in detail in section 3.1, $V(x)$ is a $L$-periodic potential, $\xi(t)$ is a white Gaussian noise of strength $2 \eta k_{B} T$, and $y(t)$ is either an unbiased $\mathcal{T}$-periodic function or an unbiased stationary random process (especially independent of $\xi(t)$ and $x(t)$ ). With respect to the load force $F$ from (3.1), we immediately focus on the case of main interest $F=0$.

According to Curie's principle (section 2.7), noise induced transport is expected when the system is permanently kept away from thermal equilibrium and does not exhibit a spatial inversion symmetry. Within the model (5.1), these requirements can be met in two basic ways: The first option is an asymmetric "ratchet-potential" $V(x)$ in combination with a perturbation $y(t)$ which is symmetric under inversion $y(t) \mapsto-y(t)$ (see section 3.2 for a precise definition), amounting to a "fluctuating force ratchet" if $y(t)$ is a random process, and to a "rocking ratchet" if $y(t)$ is periodic in $t$. The second option is a spatially symmetric $V(x)$ in combination with a broken symmetry of $y(t)$, called an "asymmetrically tilting ratchet".

A few models which go beyond the basic form (5.1) are also included in the present chapter: Before all, this concerns the discussion of photovolatic effects in section 5.2. Moreover, the influence of finite inertia is discussed in section 5.4, while twodimensional generalizations are the subject of section 5.5.

\subsubsection{Adiabatic approximation}

The simplest situation in (5.1) arises if the changes of $y(t)$ in the course of time are extremely slow 11]. Then, at any given instant $t$, the particle current has practically the same value as the steady state current (2.37) for the tilted Smoluchowski-Feynman ratchet (2.34) with a static tilt $F=y(t)$. Like in section 2.10, this so-called adiabatic approximation thus corresponds to an accompanying steady state description in which the time $t$ plays the role of a parameter.

For a periodic driving $y(t+\mathcal{T})=y(t)$, the time averaged current (3.5) in the 
adiabatic approximation thus follows as 11, 42, 52]

$$
\begin{aligned}
\langle\dot{x}\rangle & =\frac{1}{\mathcal{T}} \int_{0}^{\mathcal{T}} d t v(y(t))=\int_{0}^{1} d h v(\hat{y}(h)) \\
v(y) & :=\frac{L k_{B} T\left[1-e^{-L y / k_{B} T}\right]}{\eta \int_{0}^{L} d x \int_{x}^{x+L} d z e^{[V(z)-V(x)-(z-x) y] / k_{B} T}} \\
\hat{y}(h) & :=y(h \mathcal{T}) .
\end{aligned}
$$

Similarly as in section 2.10, it is assumed that apart from the variation of the timeperiod $\mathcal{T}$ itself, the shape of $y(t)$ does not change, i.e. $\hat{y}(h)$ is a $\mathcal{T}$-independent function of $h$. As a consequence, the right hand side of (5.2) is independent of $\mathcal{T}$, in close analogy to equation (2.57). In the zero-temperature limit $T \rightarrow 0$, one finds similarly as in 4.36 that

$$
v(y, T=0)= \begin{cases}\frac{\eta L}{\int_{0}^{L} \frac{d x}{y-V^{\prime}(x)}} & \text { if } y \neq V^{\prime}(x) \text { for all } x \\ 0 & \text { otherwise }\end{cases}
$$

For finite but very small temperatures $T$ this result is only slightly modified if $y \neq V^{\prime}(x)$ for all $x$. In the opposite case, there are ( $y$-dependent) solutions $x=x_{\max }$ and $x=x_{\min }$ of $y=V^{\prime}(x)$ with the property that $x_{\max }$ maximizes $V(x)-x y$ within the interval $\left[x_{\min }, x_{\min }+L\right]$ and $x_{\min }$ minimizes $V(x)-x y$ within $\left[x_{\max }-L, x_{\max }\right]$, cf. section 2.5.1. From (2.40)-(2.46) we can read off that

$$
\begin{aligned}
v(y) & =L\left[k_{+}-k_{-}\right] \\
& =\frac{L\left|V^{\prime \prime}\left(x_{\max }\right) V^{\prime \prime}\left(x_{\min }\right)\right|^{1 / 2}}{2 \pi \eta} e^{-\Delta V(y) / k_{B} T}\left[1-e^{-y L / k_{B} T}\right] \\
\Delta V & :=V\left(x_{\max }\right)-V\left(x_{\min }\right)-\left(x_{\max }-x_{\min }\right) y
\end{aligned}
$$

for sufficiently small temperatures such that $k_{B} T \ll \Delta V(y), \Delta V(y)-y L$.

If $y(t)$ in (5.1) is an unbiased stochastic process with an extremely large correlation time (cf. (4.2))

$$
\tau:=\frac{\int_{-\infty}^{\infty} d t\langle y(t) y(s)\rangle}{2\left\langle y^{2}(t)\right\rangle}
$$

then one obtains along the same line of reasoning as in (5.2) the adiabatic approximation 355

$$
\langle\dot{x}\rangle=\int_{-\infty}^{\infty} d y \rho(y) v(y) .
$$

Here, $\rho(y)$ is the distribution of the noise (cf. (3.11))

$$
\rho(y):=\langle\delta(y-y(t))\rangle
$$

and it is required that $\rho(y)$ does not change upon variation of the correlation time $\tau$. We have encountered this so-called constant variance scaling assumption already in section 4.1 and it is obviously the counterpart of the $\mathcal{T}$-independence of $\hat{y}(h)$ from (5.4) in the case of a periodic driving $y(t)$. 
For general analytic conclusions, the adiabatic expressions (5.2) or (5.9) are still too complicated, one has to plot concrete examples with the help of (5.3) numerically. Only in particularly simple special cases one may also be able to directly predict the direction of the current. Such an example arises if $y(t)$ can take only two possible values $\pm y_{0}$ with very rare deterministic or random flips, and $V(x)$ exhibits a very simple ratchet profile, consisting essentially of one steep and one flat slope (see e.g. figure 2.2 or 4.1). Upon increasing $y_{0}$, the condition $y \neq 0$ for all $x$ in (5.5) will be first satisfied either for $y=y_{0}$ or $y=-y_{0}$ with a resulting $T=0$ current in (5.2) or (5.9) with a sign equal to that of the flat slope. The intuitive picture is simple: Out of the two tilted asymmetric potentials $V(x) \mp y_{0} x$, one does not exhibit any extrema and thus supports a permanent downhill motion, while the other still exhibits extrema which act as motion-blocking barriers. One may go one step further and again decrease $y_{0}$ until both $V(x) \mp y_{0} x$ exhibit potential barriers and thus prohibit deterministic motion. One readily sees that the barrier induced by the steeper slope of $V(x)$ is higher than that induced by the flatter slope. With (5.6) it follows again that for weak thermal noise the current goes into the direction of the flat slope of $V(x)$. Similarly, for an asymmetrically tilting ratchet with only two possible values $y_{ \pm}$ for $y(t)$ and a symmetric potential $V(x)$, the sign of the bigger slope $y_{ \pm}$in modulus dictates the sign of the current $\langle\dot{x}\rangle$.

Numerical evaluations 111, 15, 42, 116, 182, 183, 193, 224, 228, 236, 265, 355 360] of the adiabatic expressions (5.2) or (5.9) for more complicated drivings $y(t)$ but still relatively "simple" potentials like in figures 2.2, 4.1 lead to analogous conclusions.

Another noteworthy feature arises if only small $y$-values are known to play a significant role in the expression for the adiabatic current (5.2) or (5.9). For $T=0$ it immediately follows from (5.5) that $\langle\dot{x}\rangle=0$ For $T>0$ and sufficiently small $y$, one may linearize (5.3) to obtain

$$
v(y)=y L^{2}\left[\eta \int_{0}^{L} d x e^{-V(x) / k_{B} T} \int_{0}^{L} d x e^{V(x) / k_{B} T}\right]^{-1} .
$$

Since $y(t)$ is unbiased, see (3.9) or (3.10), one recovers again $\langle\dot{x}\rangle=0$ from (5.2) or (5.9), in agreement with the general prediction from section 3.7.

\subsubsection{Fast tilting}

In the case of a stochastic process $y(t)$ with a very small correlation time (5.8) one may proceed under the assumption of constant variance scaling along the same line of reasoning like for the fast pulsating ratchet scheme in section 4.1. Thus, we can replace in leading order $\tau$ the random precess $y(t)$ by a white Gaussian noise with the same intensity $\int d t\langle y(t) y(0)\rangle=2 \tau\left\langle y^{2}(0)\right\rangle$. Like in section 4.3.2, the resulting two independent Gaussian white noises in (5.1) can be lumped into a single Gaussian white noise. We thus recover an effective Smoluchowski-Feynman ratchet, implying that in leading order $\tau$ no current $\langle\dot{x}\rangle$ is obtained the above simple type of argument to higher orders in $\tau$, such higher order results have to be derived separately for each specific type of noise $y(t)$. Similarly, for periodic perturbations $y(t)$ one finds zero current in leading order of the period $\mathcal{T}$ and one has to

\footnotetext{
${ }^{1}$ Strictly speaking, our argument is only valid for $T>0$. The conclusion, however, also remains true for $T=0$, see at the end of section 5.1 .
} 
proceed to higher orders. The main conclusion of those various expansions, reviewed in more detail in the next section, is that the current $\langle\dot{x}\rangle$ for fast tilting ratchets vanishes in leading order and depends on the detailed properties of $y(t)$ in higher orders, i.e. no simple universal results as for the pulsating ratchets in section 4.1 are accessible.

\subsubsection{Comparison with pulsating ratchets}

From section 5.0.3 we can infer as a first major difference in comparison with the pulsating ratchet scheme that for tilting ratchets, a finite current $\langle\dot{x}\rangle$ is generically observed in the limit of adiabatically slow tilting. Since in experiments it is often difficult to go beyond the adiabatic regime, this feature is an invaluable advantage of the tilting ratchet paradigm. An interesting exceptional class of asymmetrically tilting ratchets will be discussed in section 5.8.

Our second conclusion is that the "natural" current direction in fluctuating force and rocking ratchets is given by the sign of the flat potential slope. Comparison with section 4.3.1 shows that this "natural" direction is just opposite to the "natural" direction in a fluctuating potential ratchet. A similar "natural" direction can be identified for asymmetrically tilting ratchets. However, precise criteria of "simplicity" such that this natural current direction is actually realized are not available? Opposite current directions can definitely been observed in more complicated potentials $V(x)$ and also for "simple" potentials outside the adiabatic regime. Examples will be given later and can also been constructed along the lines of section 3.6.

A third major difference in comparison with the fluctuating potential ratchet model is that thermal noise is not indispensable for the occurrence of the ratchets effect provided sufficiently large tilting forces $y(t)$ appear in (5.2) or (5.9) such that a finite velocity in (5.5) is possible. This feature is of particular conceptual appeal in the case of a stationary stochastic process $y(t)$ with unrestricted support of $\rho(y)$, e.g. a Gaussian distributed noise. In the absence of the thermal noise $\xi(t)$ in (5.1) we obtain a ratchet effect for a system in a non-equilibrium environment of archetypal simplicity? see also chapter 3. Such models have been extensively studied under the label colored noise problem, see [67] for a review.

In section 5.0 .4 we have found that (within a constant variance scaling scheme) the current vanishes in leading order of the characteristic time scale in the fast tilting limit. Along a completely analogous line of reasoning one sees that for a Gaussian noise driven fluctuating potential ratchet within a constant intensity scaling scheme the current still vanishes in the white noise limit, while it remains finite for a fluctuating potential ratchet, see section 4.3 .2 (for a traveling potential ratchet this limit is not well defined). In other words, both in the fast and slow driving limits, pulsating and tilting ratchets behave fundamentally different.

\footnotetext{
${ }^{2}$ Such precise criteria would probably be very complicated (in the worst case a huge lookup table) and thus of little practical use and moreover different for any type of ratchet. On the other hand, there will be also many "complicated" examples which nevertheless lead to a "natural" current direction.

${ }^{3}$ We may always consider $y(t)+\xi(t)$ in $(5.1)$ as a single noise, stemming from one and the same non-equilibrium heat bath, but for $T \neq 0$ this viewpoint is not very "natural".
} 


\subsection{Fluctuating force ratchets}

In this section we consider the tilting ratchet scheme (5.1) with a spatially asymmetric, $L$-periodic potential $V(x)$ and a fluctuating force $y(t)$ which is given by a stationary stochastic process, symmetric under inversion $y(t) \mapsto-y(t)$ (in the statistical sense, see section 3.2), hence in particular unbiased (3.10).

Physically, this gives rise to a model of paradigmatic simplicity for a system under the influence of a non-thermal heat bath. Similarly as for the so-called "colored noise problem" [67, the setup is mainly of conceptual interest, while its direct applicability to real systems is limited, see also sections 3.4 .2 and 5.1 .2 .

As argued in the preceding section, if $y(t)$ is another Gaussian white noise then we are dealing with an effective Smoluchowski-Feynman ratchet. Hence, to obtain directed transport one either has to invoke a correlated (non-white), Gaussian or non-Gaussian noise ("colored noise"), giving rise to a so-called correlation ratche招 [265, 356], or a white, non-Gaussian noise.

As far a Gaussian colored noise is concerned, its properties are completely fixed by the first and second moments $\langle y(t)\rangle=\langle y(0)\rangle$ and $\langle y(t) y(s)\rangle=\langle y(t-s) y(0)\rangle$ 100,101. Focusing on unbiased stationary examples, the distribution is thus always given by (3.15), while the correlation $\langle y(t) y(s)\rangle$ can be chosen largely arbitrarilyf. The simplest example is Ornstein-Uhlenbeck noise with an exponentially decaying correlation (3.11).

A standard example of a non-Gaussian colored noise is the symmetric dichotomous noise from (3.12)-(3.13). A further example of interest is its generalization with three instead of two states [361], i.e. the noise $y(t)$ can take three possible values, $y(t) \in$ $\{-B, 0, B\}$. The transition rates from $\pm B$ to 0 are defined as $1 / \tau$ and the backward rates from 0 to $\pm B$ as $\lambda / \tau$,

$$
k_{ \pm B \rightarrow 0}=1 / \tau, \quad k_{0 \rightarrow \pm B}=\lambda / \tau .
$$

This so-called three state noise is thus characterized by the three parameters $B, \tau, \lambda>$ 0 . Note that the so defined $\tau$ is proportional but in general not identical to the correlation time defined in (5.8). The rather lengthy expression for the proportionality factor follows from a straightforward calculation but is of no further interest for us. In the context of the above three state noise, $\tau$ will always be understood as given by (5.12) rather than (5.8). The special case of a dichotomous noise is recovered in the limit $\lambda \rightarrow \infty$.

Finally, so-called symmetric Poissonian shot noise is defined as 178, 326 329]

$$
y(t)=\sum_{i=-\infty}^{\infty} y_{i} \delta\left(t-\tau_{i}\right),
$$

where the "spiking times" $\tau_{i}$ are independently sampled (thus Poissonian) random numbers with average interspike distance

$$
\mathcal{T}:=\lim _{k \rightarrow \infty} \frac{1}{2 k+1} \sum_{i=-k}^{k}\left(\tau_{i+1}-\tau_{i}\right) .
$$

\footnotetext{
${ }^{4}$ The same name has been introduced in [17] for a fluctuating potential ratchet in our present nomenclature.

${ }^{5}$ One obvious restriction is that the intensity $\int d t\langle y(t) y(s)\rangle$ and hence the correlation time in 5.8 must not be negative nor infinite.
} 
Furthermore, the spiking amplitudes $y_{i}$ in (5.13) are random numbers, independent of each other and of the $\tau_{i}$, distributed according to some symmetric distribution $P\left(y_{i}\right)$. Specifically, we will consider the example

$$
P\left(y_{i}\right)=\frac{1}{2 A} e^{-\left|y_{i}\right| / A}
$$

yielding a correlation of the form

$$
\langle y(t) y(s)\rangle=2 A^{2} \mathcal{T}^{-1} \delta(t-s),
$$

i.e. this type of shot noise is uncorrelated (white noise) with two model parameters $\mathcal{T}$ and $A$. Yet, in close analogy to correlated noise (cf. section 4.1 and 5.0.3), the $\mathcal{T}$-dependence of $y(t)$ is of the form $\hat{y}(t / \mathcal{T})$ with a suitably defined, $\mathcal{T}$-independent Poissonian white shot noise $\hat{y}(t)$.

Note that a similar (but asymmetric) type of Poissonian shot noise has already been encountered in (4.43)-4.45) and will later appear again in the asymmetrically tilting ratchet scheme in section 5.8.2. Throughout the present review, Poissonian shot noise (symmetric or not) will be employed as an interesting abstract example process of archetypal simplicity. For concrete applications in various contexts of electronic devices and solid state physics see for instance [137]. For models of chemical reactions and other transport processes in gases we refer to [362]. We furthermore remark that the above Poissonian symmetric shot noise can be recovered 329] as a limiting case of the three state noise (5.12) if

$$
\begin{aligned}
& \tau \rightarrow 0, \quad \lambda \rightarrow 0, \quad B \rightarrow \infty \\
& \mathcal{T}:=\tau / 2 \lambda, \quad A:=\tau B \text { fixed } .
\end{aligned}
$$

\subsubsection{Fast fluctuating forces}

We first address the case of a correlation ratchet (colored noise $y(t)$ ) in the regime of small correlation times $\tau$ in (5.8). Examples are a dichotomous noise or an OrnsteinUhlenbeck noise, cf. (3.12)-(3.15). As mentioned before (see section 5.0.4), a simple leading-order $\tau$ argument as for the pulsating ratchet scheme in section 4.1 yields the trivial result $\langle\dot{x}\rangle=0$, i.e. the correlation ratchet is in some sense reluctant to obey Curie's principle in the fast noise regime. Higher order $\tau$ contributions require a separate perturbation calculation for each type of noise $y(t)$, similar in spirit as the example in Appendix C.

The result of such perturbation calculations for various types of noises $y(t)$, among others symmetric dichotomous noise, three-state noise, and Ornstein-Uhlenbeck noise, can be written in the general form [35, 49, 355, 361, 363.

$$
\langle\dot{x}\rangle=-\frac{\tau^{3} L\left\langle y^{2}(0)\right\rangle}{\eta k_{B} T} \frac{Y_{1} \frac{\left\langle y^{2}(0)\right\rangle}{\left(k_{B} T\right)^{2}} \int_{0}^{L} d x\left[V^{\prime}(x)\right]^{3}+Y_{2} \int_{0}^{L} d x V^{\prime}(x)\left[V^{\prime \prime}(x)\right]^{2}}{\int_{0}^{L} d x e^{V(x) / k_{B} T} \int_{0}^{L} d x e^{-V(x) / k_{B} T}},
$$

where $Y_{1,2}$ are dimensionless and $\tau$-independent characteristic numbers of the specific noise $y(t)$ under consideration. For instance, for a dichotomous process one has 44, 355,363

$$
Y_{1}^{(D N)}=1, \quad Y_{2}^{(D N)}=1
$$


For Ornstein-Uhlenbeck noise one can infer from [35, 37, 265, 295, 296, 355] that

$$
Y_{1}^{(O U)}=0, \quad Y_{2}^{(O U)}=1 .
$$

For the three-state noise from (5.12) one has [361]

$$
Y_{1}^{(3)}=\left[3 \phi-\phi^{2}-1\right] / \phi^{3}, \quad Y_{2}^{(3)}=1 / \phi,
$$

where the so-called "flatness" is defined as

$$
\phi:=\left\langle y^{4}(0)\right\rangle /\left\langle y^{2}(0)\right\rangle^{2} .
$$

For the specific case of the three-state noise in (5.12) one obtains the result $\phi=1+1 / 2 \lambda$, which has to be substituted in (5.21).

The following assumptions are crucial for the validity of (5.18): (i) constant variance scaling of the colored noise $y(t)$; (ii) finite thermal noise strength $T>0$; (iii) smooth potential $V(x)$. It is not proven but may be expected as an educated guess that the general form (5.18) of the small- $\tau$ asymptotics remains valid even beyond the various examples of colored noises $y(t)$ so far covered in [35, 49, 265, 355, 361, 363.

Turning to the case of the symmetric Poissonian white shot noise (5.13), (5.15) one readily recovers the asymptotic behavior [126] for small characteristic times in (5.14) from the behavior of the three-state noise (5.18), (5.21) in the limit (5.17). Remarkably, the result is then again given by the same formula as in (5.18) if one makes the "natural" replacement

$$
\tau^{3}\left\langle y^{2}(0)\right\rangle^{2} \mapsto \mathcal{T}^{3} A^{4}
$$

and with

$$
Y_{1}^{(s h o t)}=-1, \quad Y_{2}^{(s h o t)}=0 .
$$

Our first observation in (5.18) is that $\langle\dot{x}\rangle$ vanished not only in leading order $\tau$, as already mentioned above, but also in second order, i.e. the fast fluctuating force ratchet is very reluctant to produce a current. Second, the functional dependence on the potential $V(x)$ in $(5.18)$ becomes identical to the fluctuating potential asymptotics in (4.10) when $Y_{2} \rightarrow 0$ (e.g. for shot noise) and identical to the asymptotics for the temperature ratchet in (2.58) when $Y_{1} \rightarrow 0$ (e.g. for Ornstein-Uhlenbeck noise). This comparison gives also a quantitative flavor about the necessary caution to be observed when comparing "natural" directions in fluctuation force and fluctuating potential ratchets.

Regarding the quantity $Y_{1}$ in (5.18), it has been conjectured in [35, 49] that, for a rather general class of colored noises $y(t)$, it is given by a simple function of the flatness (5.22), e.g. $Y_{1}=2-\phi$ for dichotomous and Ornstein-Uhlenbeck noise, and by (5.21) for the three-state noise (5.12). So far neither a proof nor a counterexample seems to be known. The coefficient $Y_{2}$ depends in general on additional details of the noise $y(t)$. E.g. for Gaussian noise (3.15) but with a correlation which is not given by the pure exponential decay (3.13), the flatness in $(5.22)$ is obviously always the same, while the expression for $Y_{2}$ is in general different from the one in (5.20), as can be concluded from [37,227] (see also footnote 5 on page 90 below).

The direction of the current in (5.18) is determined by the characteristics $Y_{1,2}$ of the noise $y(t)$ and the integrals over $\left[V^{\prime}(x)\right]^{3}$ and $V^{\prime}(x)\left[V^{\prime \prime}(x)\right]^{2}$. The latter fact 
makes once more explicit the warning from section 3.6 that beyond the most primitive potential shapes, there exists no simple rules and natural directions any more, the sign of $\langle\dot{x}\rangle$ depends on all the details of $V(x)$ [265]. Another surprising observation [35] is that a current inversion solely upon changing the statistical properties of the noise $y(t)$ is possible. An example [361] is the three-state noise (5.12) which in the shot noise limit (5.17) gives, according to (5.24), rise to a current direction in (5.18) opposite to that for the dichotomous noise limit $\lambda \rightarrow \infty$ (see (5.19)), at least for $\left\langle y^{2}(0)\right\rangle \gg k_{B} T$. In fact, when $\left\langle y^{2}(0)\right\rangle \gg k_{B} T$ such an inversion upon changing the noise statistics will occur for any potential $V(x)$ due to the factorization of the noise- and potentialproperties in the numerator of (5.18) and is thus of a very different nature than the inversion-tailoring procedure from section 3.6.

For the case of Ornstein-Uhlenbeck noise $y(t)$, the existence of rather simple looking potentials $V(x)$ has been first pointed out in 265] which give rise in the adiabatic limit $\tau \rightarrow \infty$ to a current $\langle\dot{x}\rangle$ in the corresponding "natural" direction (see section 5.0.5), but in the opposite direction in the small- $\tau$ limit according to (5.18), (5.20). As a consequence, a current inversion upon variation of the correlation time $\tau$ has been predicted theoretically and verified by precise numerical results in [265]. An analogous theoretical prediction and numerical verification in the case of dichotomous noise $y(t)$ is due to [250]. Considering that for simple (saw-tooth-like, but smooth) potentials $V(x)$, the "natural" current direction will definitely be recovered in the adiabatic limit $\tau \rightarrow \infty$ (cf. section 5.0.3), a current inversion as a function of the correlation time $\tau$ follows also for a three-state noise with suitably chosen parameters in (5.12) 224, see also [355, 361, 364, 365]. A similar conclusion was reached in 226] for a modified three-state noise $y(t)$ with broken symmetry by cycling through the three states in a preferential sequence (see also [336]). We remark that the three-state noise $y(t)$ from [226] is supersymmetric according to (3.40), hence $V(x)$ must not be supersymmetric (but may still be symmetric) in order that $\langle\dot{x}\rangle \neq 0$.

We recall that mere the existence of current inversions as exemplified above are just special cases of our general current inversion tailoring procedure from section 3.6. For a more detailed quantitative control of the effect, analytical approximations as exploited above are however invaluable.

We conclude our discussion of the fast potential fluctuation asymptotics with some remarks regarding the validity conditions (i)-(iii) mentioned below (5.22). First, if the potential $V(x)$ is not smooth, then the second integral in (5.18) is ill-behaving. The adequate small- $\tau$ analysis becomes much more involved and yields an "anomalous" $\tau^{5 / 2}$ leading order behavior $49,363,366$. 3aradoxically, a piecewise linear saw-tooth potential (figure 4.1), originally introduced as a stylized approximation of more realistic, smooth potentials in order to simplify the mathematics, actually makes the calculations more difficult for $\tau \rightarrow 0$. Second, we remark that while we are exclusively using here a constant variance scaling for the noise $y(t)$, in the literature on the small- $\tau$ asymptotics a constant intensity scaling is often (but not always) employed. Third, in the case $T=0$, which we excluded so far, one finds within the realm of constant variance scaling that for small $\tau$ the current $\langle\dot{x}\rangle$ approaches zero faster than any power of $\tau$ (for constant intensity scaling see [364]). 


\subsubsection{Specific types of fluctuating forces}

Beyond the fast and adiabatically slow fluctuating force limits, there has been a great variety of analytical and numerical studies. We restrict ourselves to a brief overview of the main analytical results and numerically observed effects together with the few so far suggested or actually realized experimental systems. For a more detailed discussion of the interesting special features in each particular case we refer to the cited works.

\section{Dichotomous noise}

For a dichotomous process $y(t)$ (see (3.12), (3.13)) closed, though not very transparent analytical solutions are possible for $T=0$ and arbitrary $V(x)$ [35, 193, 355, 367 and for arbitrary $T$ and piecewise linear $V(x)$ 15, 291, 355, 368 along the same lines as for the fluctuating potential scheme described in section 4.3.1.

For $T=0$ one sees from (5.5), (5.9), (3.12) that in the adiabatic limit the current vanishes for small amplitudes $\sigma$ of $y(t)$. Upon increasing $\sigma$, the current $\langle\dot{x}\rangle$ as a function of $\sigma$, sets in continuously but with a jump in its derivative when one of the two effective potentials $V(x) \mp \sigma x$ in (5.1) ceases to exhibit barriers against overdamped deterministic motion. A similar discontinuous derivative appears when the extrema of the other effective potential disappear. Upon adding in (5.1) a load force $F$ (and keeping $\sigma$ fixed), two analogous jumps in the "differential resistance" $\partial\langle\dot{x}\rangle / \partial F$ arise, while $\langle\dot{x}\rangle$ itself is always continuous. The same features are recovered [193, 195 not only in the adiabatic limit but for any finite correlation time $\tau$, basically because the noise $y(t)$ may remain with small but finite probabilities in the same state $+\sigma$ or $-\sigma$ for arbitrary long times.

If $\langle\dot{x}\rangle \neq 0$ for $T=0$, then a straightforward perturbation expansion for small but finite $T$ is possible with the expected result of small corrections to the unperturbed result $\langle\dot{x}\rangle$. More challenging is the case that $\langle\dot{x}\rangle=0$ for $T=0$, calling for a so-named singular perturbation theory for small $T$, see section 3.8. This task has been solved in 250 by a rate calculation based on WKB-type methods which become asymptotically exact for small $T$ for both, arbitrary correlation times $\tau$ and arbitrary (smooth) potentials $V(x)$. The connection between the rates obtained in this way and the current then follows as usual from (3.55), yielding a very good agreement with accurate numerical results 250.

An experimental ratchet system with additive dichotomous fluctuations has been proposed by way of combining in an electric circuit two components that will both be discussed separately in more detail below: On the one hand, an asymmetric dc-SQUID (superconducting quantum interference device) threatened by a magnetic flux gives rise to an effective ratchet-shaped potential for the phase, see [357] and section 5.6. On the other hand, a point contact with a defect, tunneling incoherently between two states, can act as a source of dichotomous noise, see 193 and section 5.8.2. Studies based on an experimental analog electronic circuit have been performed for negligibly small thermal noise $T \rightarrow 0$ both in the overdamped limit as well as in the presence of a finite inertia term $m \ddot{x}(t)$ on the right hand side of (5.1) in 194, 195]. Inertia-like effects have also been theoretically addressed, both for dichotomously fluctuating potential and fluctuating force ratchets, in [369]. 


\section{Gaussian noise}

The simplest type of Gaussian noise $y(t)$ is Ornstein-Uhlenbeck noise, characterized by an exponentially decaying correlation (3.13), (3.15). A first, numerical study of the corresponding correlation ratchet dynamics (5.1) has been reported in [11], recognizing as main difference in comparison, e.g. with dichotomous noise, the fact that even in the absence of the thermal noise $(T=0)$, a ratchet effect $\langle\dot{x}\rangle \neq 0$ arises generically for any finite intensity of $y(t)$.

The case $T=0$ has been further studied analytically for small $\tau$ in 35, 265, 295, 296] and especially in [37], indicating that even within the restricted class of Gaussian colored noises $y(t)$, the direction of the current may change solely by modifying the statistical properties of this Gaussian noise?t. This prediction has been numerically corroborated and extended to the finite- $T$ regime in [227, 370], revealing moreover multiple current inversions beyond the realm of small $\tau$. Additional details of the $T=0$ case have been studied theoretically in [131, 196, 371-373] and by means of an experimental analog electronic circuit in 196.

Very accurate numerical results over extended parameter regimes as well as two different analytical approximations for arbitrary (smooth) potentials $V(x)$ and OrnsteinUhlenbeck noise of arbitrary correlation time $\tau$ in the activated barrier crossing regime (i.e. weak noises $\xi(t)$ and $y(t))$ are contained in in [265]. These approximations exploit the connection (3.55) between the particle current $\langle\dot{x}\rangle$ and the rate expressions from a path-integral [247] and a so-called generalized unified colored noise approximation [248, 249], originally derived in the context of the so-called "resonant activation" effect. While the path integral method yields qualitatively the correct behavior over the whole $\tau$ regime, including the occurrence and location of current inversions, the generalized unified colored noise approximation is limited to small $\tau$ values, where it is superior to the path integral approach. Supplementary studies along the same lines with particular emphasize on the above mentioned accurate numerical methods and results are contained in 370,374

For tilting ratchets driven by Ornstein-Uhlenbeck noise $y(t)$, several groups have studied in detail the effect of finite inertia, i.e. if on the right hand side of (5.1) a term $m \ddot{x}(t)$ is included $1119,156,222,223,375,376$. Analytically, this problem represents a considerable technical challenge and the results of various approximative approaches are not always compatible. The upshot of those analytical as well as numerical explorations is the convincing demonstration that also the particle mass is a parameter, upon variation of which the current may change sign, i.e. a mass-sensitive particle separation is feasible. Similar conclusions have been reached in [195, 369 for dichotomous instead of Ornstein-Uhlenbeck noise $y(t)$.

\footnotetext{
${ }^{6}$ The reason is the infinite support of the distribution $\rho(f)$ in $(3.15)$ as compared to the bounded support e.g. for dichotomous noise in (3.12), thus the potential barrier cannot block completely any transport.

${ }^{7}$ For an unbiased, stationary Gaussian process, the statistical properties are completely determined by its correlation $\langle y(t) y(s)\rangle=\langle y(t-s) y(0)\rangle$. While $Y_{1}=2-\phi$ in 5.18 is always zero, $Y_{2}$ may change its sign upon modification of the correlation. The prediction from [37] is that the sign of $Y_{2}$ is given by that of $\int d t t^{2}\langle y(t) y(0)\rangle$.

${ }^{8}$ The case without a white Gaussian noise $\xi(t)$ but instead with an Ornstein-Uhlenbeck noise $y(t)$, an additional periodic (rocking) force, and possibly also a memory friction (cf. section 6.4.3) has been considered numerically in [119].
} 


\section{Shot noise}

The symmetric Poissonian shot noise (5.13) is of interest for several reasons. First, it demonstrates that the appearance of a net current in the fluctuating potential scheme (5.1) it is not necessary that the noise $y(t)$ is correlated in time [126]. Second, its "natural direction" is typically opposite to that of correlated noise $y(t)$ in the adiabatic limit. E.g. in a saw-tooth potential $V(x)$, the current direction turns out to have the same qualitative features as for the on-off saw-tooth potential [126] treated in section 4.2 if one identifies the characteristic time $\mathcal{T}$ from (5.14) with the correlation time in the on-off scheme. An intuitive explanation of this prima facie astonishing similarity follows from the discussion of the three-state noise in [35] in combination with its shot noise limiting behavior according to (5.17). Since for shot noise there is no correlation time, and the noise distribution $\rho(y)$ is not well defined, an adiabatic limit in the sense of (5.9) does not exist. The regime of a slow time scale $\mathcal{T}$ in (5.14) is therefore of a fundamentally different nature. Again analogous to the on-off scheme, one finds 126 that the current approaches zero as $\mathcal{T}$ becomes very large (both for constant variance and constant intensity scaling).

\subsection{Photovoltaic effects}

In this section we discuss experimental ratchet systems which cannot be realistically captured by the simple model (5.1) but are is physically closely related to it.

In non-centrosymmetric materials, photocurrents are induced by short-wavelength irradiation (optical or x-ray illumination) in the absence of any externally applied fields [377]. Experimental observations of this so-called photovoltaic effectf in ferroelectrics, piezoelectrics, and pyrroelectrics such as $\mathrm{BaTiO}_{3}$ or $\mathrm{LiNbO}_{3}$ can be traced back at least to the mid 60-s. The basis of its correct theoretical explanation was laid 1974 by Glass, von der Linde, and Negran [27], recognizing that it is not a surface or interface effect in contrast e.g. to photovoltaic effects occurring in n-p junctions (see sections 6.1 and 8.4) - but rather a bulk phenomenon with the asymmetry of the crystal lattice $\square$ playing a central role. Furthermore, they already touch upon the points that the absence of thermal equilibrium is another crucial precondition, that the effect should be a general

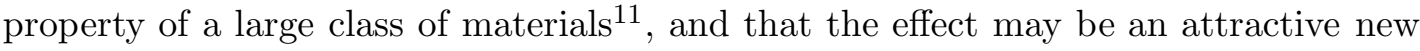
method of energy conversion in large-area pyroelectric polymers or ceramics, acting e.g. as "solar cells".

These basic ideas have been subsequently developed into a full-fledged theory by Belinicher, Sturman, and others. Several hundred experimental and theoretical papers on the subject are reviewed in [28, 29] and various general conclusions therein are remarkably similar to those of our present paper. For instance, the counterpart of the Smoluchowski-Feynman Gedankenexperiment in this context corresponds to the question why a steady state photovoltaic effect cannot exist under isotropic thermal

\footnotetext{
${ }^{9}$ Practically synonyms are "photorefractive effect" and "photogalvanic effect".

${ }^{10}$ Most of these systems exhibit a spatial periodicity, but this is de facto not an indispensable prerequisite in this context.

${ }^{11}$ Examples are monocrystalline piezoelectric materials, such as ferroelectric ceramics, or liquids and gases showing natural optical activity due to a chirality of their constituent molecules. More recent systems are provided by asymmetric semiconductor superlattices and heterostructures [37\&].
} 
blackbody irradiation. To answer such questions it is pointed out of

"gradients in concentration, temperature, or light intensity ... the current direction is controlled ... by the internal symmetry. It constitutes the generation of a directed current in a uniform medium on homogeneous illumination ... in any medium (without exception) that lacks a center of symmetry ... The absence of a center of symmetry ... results in a current in virtually any nonequilibrium stationary state. There is no current in thermodynamic equilibrium, in accordance with the second law of thermodynamics ... Under the nonequilibrium conditions provided by illumination, that detailed balancing mechanism is violated and the asymmetry in the elementary processes gives rise to a current ... The photovoltaic effect is a kinetic effect and thus has various extensions. Uniform illumination in the absence of a center of symmetry may produce not only an electrical current but also fluxes of other quantities: heat (photothermal effect), neutral particles, spin, etc. On the other hand, light beams do not exhaust the nonequilibrium sources. ... The disequilibrium may be not only due to light but to sound or to colliding or isotropic particle fluxes etc."

Another point already recognized in various studies during the 70-s and reviewed in [28, 29] is the fact that the photovoltaic effect is a nonlinear effect in the irradiation field amplitude, no current arises within the realm of linear response (cf. section 5.1.1). Furthermore, current reversals upon changing the frequency or polarization of the irradiation [159, 160] and upon changing the temperature [160] have been observed in this context.

The microscopic theoretical analysis is conducted in terms of electron scattering processes in solids [28, 29] and goes beyond our present scope. Though such an approach has little in common with our present working model (5.1), it is remarkable that veritable one-dimensional effective ratchet potentials exactly like in figure 2.1 are appearing in the discussion along these lines. We mention that it is not immediately obvious whether the effects of the irradiation, treated on an adequate quantum mechanical level, should be associated with a fluctuating force or rather with a rocking ratchet scheme: On the one hand, besides the direct interaction with the electrons, there may also be non-negligible effects of the irradiation on the host material, giving typically rise to a fluctuating potential ratchet mechanism [13]. On the other hand, the naive viewpoint that a signal, which is typically a monochromatic electrical wave, induces an electrical current suggests that the classification as a rocking ratchet - as adopted in the following - may be justified.

The photovoltaic effect is practically exploited in holography, beam amplification and correction, wavefront reversal etc. [29]. Basic research activity has somewhat decreased in recent years, focusing e.g. on the so-called mesoscopic photovoltaic effect, where random impurities in conductors or microjunctions imitate local symmetry breaking [379, 380], on x-ray induced giant photovoltaic effects [381], and on photovoltaic effects in asymmetric semiconductor heterostructures and superlattices 378.

Another variation of the photovoltaic effect has been theoretically studied in [382, 383]. Namely, in a mesoscopic, disordered normal-metal ring, a breaking of the

\footnotetext{
${ }^{12}$ In the following we are quoting from [29], but most of these statements can be found already in $[28]$.
} 
inversion symmetry can be achieved by a static magnetic flux threatening the ring, which survives even after averaging over the quenched disorder of the individual samples. As theoretically predicted in [382, 383], in such a setup the non-linear response to an additional high-frequency electromagnetic field is a directed ring-current. While somewhat similar "persistent currents" may also exist at thermal equilibrium, i.e. in the absence of the high-frequency field, only away from equilibrium these currents can be exploited to do work, i.e. we are dealing with a veritable ratchet effect. Note that the basic ingredients are remarkably similar to the SQUID ratchet systems from sections 5.3.3 and 5.6, but the detailed physical mechanisms are completely different.

Finally, worth mentioning in this context is also the generation of directed photocurrents in undoped, bulk semiconductors with an intact centrosymmetry by adjusting the relative phases of two optical beams at frequencies $\omega$ and $2 \omega$, see 384 388] and further references therein and also the discussion at the end of section 8.3 below. Such a modified photovoltaic effect leads us beyond the realm of the rocking ratchet scheme and will be treated in more detail under the label asymmetrically tilting ratchets in sections 5.8.1 and 8.3.

\subsection{Rocking ratchets}

In this section we address the tilting ratchets dynamics (5.1) with an $L$-periodic, asymmetric potential $V(x)$ and a $\mathcal{T}$-periodic, symmetric external driving force $y(t)$.

\subsubsection{Fast rocking limit}

In contrast to the slow rocking limit (adiabatic approximation), the regime of very high frequencies has turned out to be rather obstinate against analytical approximations or intuitive explanations. Attempts have been made [228, 268, 269] but cannot be considered as fully satisfactory. Numerical results, on the other hand, show [42] as a quite remarkable feature that in the fast rocking regime, the "natural" current direction (i.e. the one realized for "simple" potentials $V(x)$ sufficiently similar to the asymmetric saw-tooth potential from figure 4.1) is just opposite to the one for slow rocking. In order to finally conclude this issue, we sketch in the following the main steps of an analytical solution of the fast rocking asymptotics (details of this calculations will be presented in [389]).

Under the assumption that the $\mathcal{T}$-periodic function $y(t)$ in (5.1) is of the form (5.4), the asymptotics of the current $\langle\dot{x}\rangle$ in (5.1) for small $\mathcal{T}$ can be in principle determined along the same lines as in Appendix C. In practice, the calculations become extremely tedious since, as we will see, to obtain the first non-trivial contribution to the current, one has to go up to the 4 th order in $\mathcal{T}$. Things can be simplified a lot by mapping (5.1) onto an equivalent improper traveling potential ratchet dynamics (cf. (4.32)) as follows: With the definition

$$
X(t):=x(t)-\frac{\mathcal{T}}{\eta} \hat{y}_{1}(t / \mathcal{T})
$$

where (cf. (5.4))

$$
\begin{aligned}
\hat{y}_{0}(h) & :=\hat{y}(h)=y(h \mathcal{T}) \\
\hat{y}_{i}(h) & :=\int_{0}^{h} d s \hat{y}_{i-1}(s)+\int_{0}^{1} d s s \hat{y}_{i-1}(s), \quad i=1,2, \ldots
\end{aligned}
$$


one readily finds from (5.1) that

$$
\eta \dot{X}(t)=-V^{\prime}\left(X(t)+\frac{\mathcal{T}}{\eta} \hat{y}_{1}(t / \mathcal{T})\right)+\xi(t) .
$$

Since the relations $\hat{y}_{i}(h+1)=\hat{y}_{i}(h)$ and $\int_{0}^{1} d h \hat{y}_{i}(h)=0$ are fulfilled for $i=0$, it follows with (5.27) by induction that the same relations are respected for $i=1,2, \ldots$ Using the self-averaging property (3.5) of the particle current, we can thus infer from (5.25) that

$$
\langle\dot{x}\rangle=\langle\dot{X}\rangle
$$

After expanding on the right hand side of (5.28)

$$
V^{\prime}\left(X(t)+\frac{\mathcal{T}}{\eta} \hat{y}_{1}(t / \mathcal{T})\right)=\sum_{k=0}^{\infty} \frac{V^{(k+1)}(X(t))}{k !}\left[\frac{\mathcal{T} \hat{y}_{1}(t / \mathcal{T})}{\eta}\right]^{k}
$$

one sees that in comparison with (5.1) we have "gained" one order of $\mathcal{T}$, the "perturbation" in (5.30) is of leading order $\mathcal{T}$ only. Due to this simplification, the approach from Appendix $\mathrm{C}$ is now applicable with a reasonable effort and yields the leading- $\mathcal{T}$ result 389]

$$
\begin{aligned}
\langle\dot{x}\rangle & =\frac{2 \mathcal{T}^{4} L Y \int_{0}^{L} d x V^{\prime}(x)\left[V^{\prime \prime \prime}(x)\right]^{2}}{\eta^{5} \int_{0}^{L} d x e^{V(x) / k_{B} T} \int_{0}^{L} d x e^{-V(x) / k_{B} T}} \\
Y & :=\int_{0}^{1} d h\left[\hat{y}_{2}(h)\right]^{2} .
\end{aligned}
$$

Here, we have exploited that $y(t)$ in (5.1) is symmetric (cf. (3.17)), otherwise additional terms of order $\mathcal{T}^{4}$ would appear in (5.31), see section 5.8.1 below.

Our first conclusion from (5.31) is that the fast rocking ratchet is exceedingly reluctant to produce a current, all contribution up to the order $\mathcal{T}^{3}$ are zero. This fact suggest that also a simple intuitive explanation of the current direction may be very difficult to figure out. Second, for sufficiently simple (saw-tooth-like but smooth) potentials $V(x)$, the sign of the current in (5.31) is dictated by that of the steeper slope of $V(x)$, and this independently of any further details of the driving $y(t)$. Our result (5.31) thus correctly reproduces the numerical observation [42] that the "natural" current direction of the fast and slow rocking ratchets are opposite. In other words, a current inversion upon variation of $\mathcal{T}$ is typical ("natural") in rocking ratchet systems at finite temperatures $T>0$.

We finally remark that - much like in the approximation (2.58) for the temperature ratchet - the limits $\mathcal{T} \rightarrow 0$ and $T \rightarrow \infty$ do not commute, i.e. (5.31) is not valid for a fixed (however small) $\mathcal{T}$ if one lets $T \rightarrow \infty$, cf. section 3.7. In the special case of a fast sinusoidal driving $y(t)$ with asymptotically small amplitude our result (5.31) reproduces the one from 269]. Also worth noting is that (5.31) is strictly quadratic in the driving amplitude (see (5.32) ). Deviations from this strictly quadratic behavior are expected only in the next-to-leading order $\mathcal{T}$ contributions that have been neglected in (5.31). For this reason, the limit of asymptotically large driving amplitudes can once again not be interchanged with the fast driving limit $\mathcal{T} \rightarrow 0$. 


\subsubsection{General qualitative features}

A first remarkable feature of a periodically rocked ratchet dynamics (5.1) occurs if in the deterministic limit $(T \rightarrow 0)$. Namely, the current $\langle\dot{x}\rangle$ as a function of the rocking amplitude $y(t)$, but also as a function of other parameters, displays a complex structure of constant "plateaux" which are separated by discontinuous jumps [11, 39, 42, 51, 228, 357, 371]. For a qualitative explanation we first note that the current $\langle\dot{x}\rangle$, understood as a long time average (3.5), is independent of the initial condition ${ }^{[3} x\left(t_{0}\right)$ [39]. The emergence of the current-plateaux can be analytically understood in detail for a sawtooth potential $V(x)$ and a driving which periodically jumps between a few discrete values [39,228], while in more complicated cases numerical solutions must be invoked [11, 42, 357]. Very loosely speaking, the deterministic dynamics (5.1) with periodic $y(t)$ and $\xi(t) \equiv 0$ is equivalent to a two-dimensional autonomous dynamics and thus admits as attractors generalized fixed points and periodic orbits, where the word "generalized" refers to the fact that we identify $x$ and $x+L$ as far as the attracting set is concerned. Thus, in the long time limit, that is, after transient effects have died out, the particle is displaced by some multiple $m$ of the spatial period $L$ after a certain multiple $n$ of the time period $\mathcal{T}$, i.e.

$$
\langle\dot{x}\rangle=(L / \mathcal{T})(m / n)
$$

Remarkably, though $\langle\dot{x}\rangle$ is independent of the initial condition, several generalized periodic attractors (with the same $m / n$ ) may still coexist 42]. The structural stability of these attractors implies that as a function of various model parameters, the ratio $m / n$ and thus $\langle\dot{x}\rangle$ jumps only at discrete points and is constant in between. In other words, a kind of locking mechanism is at work, closely related to the one responsible for the Shapiro steps in symmetric potentials with an extra tilt $F$ on the right hand side of (5.1) 390, 391]. Further intriguing features, like the appearance of Devil's staircases of current-plateaux or current reversals of $\langle\dot{x}\rangle$ as a function of the driving amplitude $y(t)$, are discussed in detail in [39, 42, 51, 228].

Upon including the thermal noise in (5.1), the details of the complex behavior of $\langle\dot{x}\rangle$ as a function of various model parameters is washed out. While for simple, sawtooth-type potentials $V(x)$ like in figures 2.2, 4.1 and not too large rocking amplitudes, the deterministic $(T=0)$ current $\langle\dot{x}\rangle$ is known [11, 39, 42, 228] to always exhibit the same direction, a current inversion for sufficiently fast driving sets in as soon as a finite amount of thermal noise $(T>0)$ is added, as confirmed by our perturbative result (5.31).

If the deterministic current $(T=0)$ vanishes, then for weak thermal noise (small $T)$ an activated barrier crossing problem arises which can be reduced to an escape rate problem via (3.55). In general, analytical progress requires technically sophisticated path-intergal and WKB-type singular perturbation methods which are beyond our present scope, see also section 3.8.

Both, in the limits of small and large driving amplitudes one can readily see that the current approaches zero. Hence, there must be an "optimal" amplitude in between for which the current is maximized. Typically, the dependence of $\langle\dot{x}\rangle$ upon the amplitude is roughly speaking of a single-humped shape [39,42], onto which, however, the previously

\footnotetext{
${ }^{13}$ This property readily follows from the fact that $x\left(t_{0}\right)$ and $x\left(t_{0}\right)+L$ obviously lead to the same $\langle\dot{x}\rangle$ and that different trajectories $x(t)$ cannot cross each other [39].
} 
described (non-monotonic) fine-structure for small or zero thermal noise intensity is superimposed.

\subsubsection{Applications}

An experimental realization of a rocking ratchet system has been reported in [392]: A mercury drop in a capillary with a periodically but asymmetrically varying diameter is subjected to an oscillating external electrical force of electrocapillary nature. While thermal fluctuations are negligible and the experimental situation is at most qualitatively captured by the one-dimensional model dynamics (5.1), besides the directed transport itself also the "resonance-like" dependence of the current $\langle\dot{x}\rangle$ upon the rocking amplitude, as predicted theoretically, has indeed been observed in the experiment.

Several further experimental realizations of the rocking ratchet scheme have been proposed: In 357 it has been demonstrated that the phase across an asymmetric SQUID threatened by a magnetic flux may be modeled by a rocking ratchet dynamics. For more details we refer to section 5.6 below.

A second realization of the rocking ratchet scheme has been suggested in [393]: The proposed system consists of a one-dimensional ${ }^{\text {Ma }}$ parallel array of Josephson-junctions with alternating critical currents and junction areas in the overdamped limit, see also section 9.1. In such a system, it can be shown that the relevant soliton-type solutions (also referred to as kinks, vortices, or fluxons) are approximately governed by a one-dimensional overdamped dynamics in an effective pinning potential which can be chosen ratchet-shaped. In other words, such a Josephson kink can be considered as quasi-particle (endowed with effective mass, velocity, interaction with other kinks, and other particle-like properties) moving in an effective one-dimensional ratchet-potential along the array and can be observed by measuring the time- and space-resolved dc voltage along the array. Taking into account an external periodic driving and thermal fluctuations, a rocking ratchet setup is thus recovered. The technical details of the problem are rather involved and finally require a numerical evaluation, see [393] for more details. Significant experimental progress towards a realization of the ratchet effect in such sorts of Josephson-junction arrays has been accomplished in [394]. A modification, based on a continuous, one-dimensional long Josephson junction (of annular shape), has been put forward in 290]. An effective ratchet potential for the kink dynamics emerges either by applying an external magnetic field and choosing a properly deformed shape of the annular Josephson junction or by modulating its width. A further option is to deposite a suitably shaped "control line" on top of the junction in order to modulate the magnetic flux through it [395]. In either way, not only rocking ratchets - as in [393, 394] - but also fluctuating potential ratchets (not necessarily overdamped) can be realized 2290,395. Further theoretical as well as experimental studies along closely related lines by several groups are currently in progress, see also sections 5.2 and 8.4 .

As a third realization of the rocking ratchet scheme, it has been proposed in [396] that the application of an alternating current to a superconductor, patterned with an asymmetric pinning potential, can induce a systematic directed vortex motion. Thus, by an appropriate choice of the ratchet-shaped pinning potential, the rocking ratchet scheme can be exploited to continuously remove unwanted trapped magnetic flux lines out of the bulk of superconducting materials. Quantitative estimates [396] show that

\footnotetext{
${ }^{14}$ Practically, a closed loop topology can replace the straight periodic setup of infinite length.
} 
thermal fluctuations are practically negligible in this application of the rocking ratchet model (5.1). For a two-dimensional version [397] of the same idea see section 5.5.

Finally, it has been predicted [237] within a simplified hopping-model (activated barrier crossing limit) for a crystalline surface, consisting of atomically flat terraces and monoatomic steps, that by application of an ac-field a surface-smoothening can be achieved due to an underlying rocking ratchet mechanism. First experimental findings which can be attributed this theoretically predictied effect are due to [398.

For additional experimental realizations see also section 8.4.

\subsection{Influence of inertia and Hamiltonian ratchets}

The rocking ratchet dynamics (5.1) supplemented by a finite inertia term $m \ddot{x}(t)$ on the right hand side is not only of experimental interest (cf. the asymmetric SQUID model in section 5.6 below) but exhibits also interesting new theoretical aspects 5 . Without the noise $\xi(t)$, the periodically driven deterministic dynamics is equivalent to a three-dimensional autonomous dynamics and thus in general admits chaotic attractors in the "generalized" sense specified at the beginning of this section. Numerical simulations [170, 230, 231, 399, 400] show that a chaotic behavior is indeed realized in certain parameter regions of the model. As another crucial difference in comparison with the overdamped case, the current in the long time average (3.55) in general still depends on the initial conditions [170, 221].

As a function of various model parameters, the current shows a still much more complex behavior than in the overdamped case, including multiple inversions even for a "simple" potential-profile like in figure 2.2. For very weak damping, the sign of the current is in fact predominantly opposite to that in the overdamped limit 170. These general features of $\langle\dot{x}\rangle$ are expected to be robust also against a certain amount of noise. The same is not expected for further interesting details of the deterministic dynamics reported in [170, 230, 231, 399 401], some of them strikingly reminiscent of previous findings in the context of deterministic diffusion in symmetric one-dimensional maps 402 408.

Of significant conceptual interest is the noiseless case in the limit of vanishing dissipation, i.e. a conservative (Hamiltonian) deterministic rocking ${ }^{10}$ ratchet dynamics

$$
m \ddot{x}(t)=-V^{\prime}(x(t))+y(t) .
$$

The salient difference in comparison with a dissipative system is the time-inversion invariance provided the time-periodic driving $y(t)=y(t+\mathcal{T}$ ) satisfies (after an irrelevant shift of the time origin) the symmetry condition 215, 221]

$$
y(-t)=y(t)
$$

see also below 77 equation (3.41). Another basic feature is the generic appearance of (Hamiltonian) chaos with its complicated hierarchical fine structure of disjoint stochastic (chaotic) layers, islands, KAM-tori etc. 4110 412]. As a consequence, the behavior

\footnotetext{
${ }^{15}$ Regarding the issue of finite inertia in traveling potential ratchets, Seebeck ratchets, fluctuating force ratchets, and quantum ratchets see sections 4.4.1, 6.1, 5.1.2, and 8.1, respectively

${ }^{16}$ A Hamiltonian generalized traveling potential ratchet model has been considered in [409].

${ }^{17}$ Note that in the present context of Hamiltonian ratchets the word "rocking ratchet"- unlike in the rest of this review - is not necessarily reserved for symmetric drivings $y(t)$, i.e. $y(t)$ is $\mathcal{T}$-periodic but need not satisfy (3.17).
} 
of the system depends in general on the initial conditions 18 unless one is in the limiting case of strong (hyperbolic) Hamiltonian chaos [165, 409, 413, 414]. Strictly speaking, this case is not generic but it is often adopted as an approximation for sufficiently strong perturbations of an integrable system with initial conditions in that stochastic layer which contains $\dot{x}$-values of either sign. While diffusive transport with its intriguing anomalous features (e.g. so-called Lévy flights) has been analyzed in great detail 410 412, our understanding of directed transport in such a system with broken symmetry is considerably less well developed.

Under the assumptions that the symmetry (5.35) is respected it has been predicted in 221 that $\langle\dot{x}\rangle=0$ provided the initial condition $x(0), \dot{x}(0)$ is part of a stochastic layer which also contains an initial condition with $\dot{x}(0)=0$. Especially, this prediction is independent of whether the potential $V(x)$ is asymmetric or not. The basic reason is that such a trajectory $x(t)$, due to ergodicity reasons, gives on the one hand rise to the same average current (3.5) as its time inverted counterpart $z(t):=x(-t)$, i.e. $\langle\dot{x}\rangle=\langle\dot{z}\rangle$. On the other hand, one also concludes that $\dot{z}(t)=-\dot{x}(-t)$ and thus $\langle\dot{x}\rangle=-\langle\dot{z}\rangle$. As a consequence, it follows [221] that $\langle\dot{x}\rangle=0$. A similar conclusion holds 221] if the symmetry conditions from section 3.2 are respected by the potential $V(x)$ and the periodic driving $y(t)$ (cf. equations (3.16) and (3.17)). Accordingly, the symmetry condition (5.35) may be considered in some sense as the Hamiltonian counterpart of the supersymmetry concept for overdamped systems (see section 3.5.4). These different symmetries have been explored in quantitative detail in 215] by means of a kinetic Boltzmann-equation approach with special consideration of the weak and strong damping regimes. Returning to the limit of a Hamiltonian rocking ratchet, we can conclude that if neither of the above mentined symmetry conditions is satisfied then the occurrence of a finite current $\langle\dot{x}\rangle$ (ratchet effects) is generically expected (and numerically observed) 166, 215, 221, 415].

On the other hand, if the initial condition $x(0), \dot{x}(0)$ is not part of a stochastic layer which also contains an initial condition with $\dot{x}(0)=0$ then generically $\langle\dot{x}\rangle \neq 0$ even if the symmetry conditions (5.35) or (3.16) and (3.17) are respected. Examples with a finite current in spite of the symmetry property (5.35) are discussed in [165, 413, 414] (see also the previous footnote).

Though it may be difficult in practice, in principle the entire phase space of the Hamiltonian dynamics (5.34) can be decomposed into its different ergodic components 5 , each of them characterized by its own particle current $\langle\dot{x}\rangle$. Next we observe 413, 414] that the "fully averaged particle" current according to the uniform (microcanonical) phase space density can be written as

$$
\int_{0}^{\mathcal{T}} d t \int_{0}^{L} d x \int_{-\infty}^{\infty} d p \dot{x}=\lim _{p_{0} \rightarrow \infty} \int_{0}^{\mathcal{T}} d t \int_{0}^{L} d x \int_{-p_{0}}^{p_{0}} d p \frac{\partial H}{\partial p}
$$

cf. sections 2.4 and 3.1. Since the Hamiltonian of the dynamics (5.34) is $H=p^{2} / 2 m+$ $V(x)-x y(t)$ it follows that the microcanonically weighted average velocity over all

\footnotetext{
${ }^{18}$ The dependence of the current $(3.5)$ on the initial conditions $x\left(t_{0}\right), \dot{x}\left(t_{0}\right)$, and especially on the "initial phase" $t_{0}$ in $y\left(t_{0}\right)$ is obvious in the special case that $V^{\prime}(x) \equiv 0$ in (5.34). Though this special case is untypical in that it does not exhibit chaos it still captures some of the essential physics of the general case.

${ }^{19}$ In the typical case, some of them are regular and some of them are chaotic. Furthermore, the borderlines between them are the intact KAM tori. Their number is infinite and they are arranged in a very complicated hierarchical pattern 410 412.
} 
ergodic components in (5.36) is equal to zero 413, 414]. An immediate implication of this "sum rule" is that a necessary requirement for directed transport is a mixed phase space since the microcanonical distribution is the unique invariant (reduced) density in this case and is always approached in the long time limit. In other words, even in the absence of the above mentioned symmetries, systems with strong (hyperbolic) chaos do not admit a ratchet effect 413, 414.

While in 221,415] the above mentioned Lévy flights are proposed as the main reason for directed transport in Hamiltonian ratchets, the emphasize in 413, 414 is put on the picture that transport in the chaotic layers has its origin in the "unbalanced" currents within the regular islands. The situation in systems with a more than two-dimensional phase space (bringing along Arnold diffusion) has so far not been considered at all.

\subsection{Two-dimensional systems and entropic ratchets}

By explicitly keeping the dynamics that governs the driving $y(t)$ or $f(t)$ in the basic ratchet model dynamics (3.1) - independently of whether a back coupling is absent (see section 3.4.2) or present (see section 7.3.1) - one trivially ends up with a twodimensional system. In this section, however, genuine vectorial generalizations of the basic model (3.1) are considered. The simplest case of such a two-dimensional ratchet system consists of two completely independent equations of the form (5.1), one for each spatial dimension $x_{1}$ and $x_{2}$. Pro forma, one may then define a common total potential $V\left(x_{1}, x_{2}\right)$ as the sum of the two individual potentials. Such a system offers the possibility to separate particles with different ratios $\left\langle\dot{x}_{1}\right\rangle /\left\langle\dot{x}_{2}\right\rangle$ according to their traveling direction in the $x_{1}-x_{2}$-plane.

A more complicated situation arises if the dynamics involves a non-trivial common potential $V\left(x_{1}, x_{2}\right)$, periodic and/or asymmetric in only one or both arguments. An example of this kind (periodic in one component only) has been treated already in the context of Feynman's ratchet in equations (6.4)- 6.7), see also [416]. Another example (periodic in both components) which, instead of the usual linear directed transport, leads to a permanent circular motion of particles, has been worked out in [117, see also 418,419. In fact, by giving up the requirement of a simple periodicity of the system along any straight spatial direction, it should be in principle possible to direct particles along arbitrarily pre-assigned pathways in properly designed two-dimensional systems 420,421, possibly even along different routes for different species of particles with identical seeds.

In 116] a two-dimensional potential landscape $V\left(x_{1}, x_{2}\right)$ was considered which consists of one straight "valley" along the $x_{1}$-axis and periodically repeated "side valleys" of finite length (dead ends). If the angle between those side-valleys and the $x_{1}$-axis is different from $\pm \pi / 2$ then the spatial symmetry along the $x_{1}$-direction is broken and a time-periodic rocking force generically induces a finite current $\left\langle\dot{x}_{1}\right\rangle$. Since this ratchet effect will occur even if there are no potential barriers along the $x_{1}$-axis, i.e. $V\left(x_{1}, x_{2}=0\right)=$ const., the name entropic ratchet has been coined for this system 116. Moreover, if an additional bias $F$ is applied along the $x_{1}$-axis, a non-monotonic behavior of $\left\langle\dot{x}_{1}\right\rangle$ as a function of $F$ may result [116]. This so-called negative differential resistance has also been previously observed in the closely related context of networks with dead-ends, see [422] and further references therein. Very similar two-dimensional entropic ratchet schemes have been proposed in [423 for the purpose of separating DNA molecules (see also 424 427] and section 5.8.1), in [397] for the purpose of pumping, 
dispersing, and concentrating fluxons in superconductors by electrical ac-currents (cf. section 5.3.3), and in [428] for the purpose of rectifying electronic currents with the help of the Coulomb blockade effect, see also 429.

Another two-dimensional rocking ratchet scheme is obtained by choosing a potential $V\left(x_{1}, x_{2}\right)$ which has basically the effect of a two-dimensional, periodic array of obstacles ("scatterers"). The spatial symmetry is broken by the shape of the single obstacles, in the simplest case a triangle. In its simplest form, such a setup can be imagined as a Galton-board-type device with a broken spatial ("left-right-") symmetry. This basic idea has been put forward already in the context of the photovoltaic effect in non-centrosymmetric materials, see section 5.1. For the purpose of separating macromolecules such as DNA, two-dimensional arrays of obstacles ("sieves") have been proposed and quantitatively analyzed in [146, 283, 419, 430 432]. The technological feasibility of such sieves - however with symmetric obstacles - has been demonstrated already before these works in 433]. An experimental implementation of the same basic concept has been realized in 434 for the purpose of transporting and separating phospholipid molecules in a two-dimensional fluid bilayer. In contrast to other standard separation methods, such a rocking ratchet system is re-usable and enables continuous operation.

Experimentally, transport of electrons in two-dimensional periodic arrays of triangular antidot scatterers under far-infrared irradiation has been demonstrated in [435]. With an approximative classical description of the system being justified in the considered parameter regime, essentially a two-dimensional rocking ratchet scheme is thus recovered.

A further two-dimesional SQUID ratchet system will be treated in section 5.6 below. Also the experimental ratchet devices described in section 4.2.1 and at the end of sections 4.4 .2 and 8.4 - though admitting suggestive and rather faithful effective one-dimensional descriptions - are strictly speaking of two-dimensional character. A three-dimensional ratchet dynamics is discussed in section 6.6. Further models with two degrees of freedom are treated in sections 6.5 and 7.6 .

\subsection{Rocking ratchets in SQUIDs}

In the theoretical work [357] it has been demonstrated that the phase across an asymmetric SQUID (superconducting quantum interference device) threatened by a magnetic flux may be modeled by a one-dimensional rocking ratchet dynamics (cf. section 5.3.3). The starting point is the standard RSJ model (resistively shunted junction model, also called Steward-McCumber model) for the phase difference $\varphi$ of the macroscopic quantum mechanical wave function across a conventional Josephson junction

$$
\frac{\Phi_{0} C}{2 \pi} \ddot{\varphi}(t)+\frac{\Phi_{0}}{2 \pi R} \dot{\varphi}(t)+I_{c} \sin \varphi(t)=I(t)+\xi(t),
$$

where $C, R$, and $I_{c}$ are the capacitance, resistance, and critical current of the junctions, $I(t)$ is the electrical current flowing through the junction, and $\Phi_{0}:=h / 2 e$ is the flux quantum. Thermal fluctuations are modeled by unbiased Gaussian white noise $\xi(t)$ of strength $2 k_{B} T / R$. For the total phase difference across a series of two identical such Josephson junctions one recovers 357 the same equation (5.37) except that $\varphi(t)$ is replaced by $\varphi(t) / 2$ and the noise strength $2 k_{B} T / R$ by $k_{B} T / R$. Next, one considers a SQUID with the usual "loop"-geometry, formed by two conducting "arms" in parallel, 
but with two identical Josephson junctions in series in one "arm", and a third junction with characteristics $C^{\prime}, R^{\prime}$, and $I_{c}^{\prime}$ in the other "arm". The total current $I_{\text {tot }}$ through the conducting loop follows by adding the currents through both arms. Under the assumption that the loop inductance is much smaller than $\Phi_{0} /\left(I_{c}+I_{c}^{\prime}+I_{\text {tot }}(t)\right.$ ) (see also the discussion below (5.51) below), the equation for the total phase difference $\varphi$ across the loop is then governed by the equation [357

$$
\begin{aligned}
& \frac{\Phi_{0}}{2 \pi}\left(\frac{C}{2}+C^{\prime}\right) \ddot{\varphi}(t)+\frac{\Phi_{0}}{2 \pi}\left(\frac{1}{2 R}+\frac{1}{R^{\prime}}\right) \dot{\varphi}(t)=-V^{\prime}(\varphi(t))+I_{\mathrm{tot}}(t)+\xi_{\mathrm{tot}}(t) \\
& V(\varphi):=-\frac{I_{c}}{2} \cos (\varphi / 2)-I_{c}^{\prime} \cos \left(\varphi+2 \pi \Phi / \Phi_{0}\right)
\end{aligned}
$$

where $\Phi$ is the total magnetic flux threatening the loop and where $\xi_{\text {tot }}(t)$ is a Gaussian white noise with correlation

$$
\left\langle\xi_{\text {tot }}(t) \xi_{\text {tot }}(s)\right\rangle=2 k_{B} T\left(\frac{1}{2 R}+\frac{1}{R^{\prime}}\right) \delta(t-s) .
$$

The noise- and time-averaged "phase current" $\langle\dot{\varphi}\rangle$ is connected to the averaged voltage $U$ across the loop according to the second Josephson equation [357]

$$
U=\frac{\Phi_{0}}{2 \pi}\langle\dot{\varphi}\rangle
$$

and thus directly accessible to an experimental measurement. In other words, for appropriately chosen external currents $I_{\text {tot }}(t)$ and (static) magnetic fields, a rocking ratchet dynamics is recovered from (5.38), which is in particular of the overdamped form (5.1) if $I_{c} R^{2} C, I_{c}^{\prime} R^{\prime 2} C^{\prime} \ll \Phi_{0}$, cf. section A.4 in Appendix A. Potentials (5.39) with additional Fourier modes may be obtained by more complicated SQUIDs with additional "arms" in parallel.

Next we turn to one of the first systems for which a ratchet effect has been theoretically described and experimentally measured [25,26]. While these early works focus on the realm of adiabatically slow rocking, the extension beyond this regime has been realized experimentally very recently in [182, 183]. The setup consists of the following two-dimensional modification of the above described rocking ratchet SQUID system (5.37)-(5.41): The starting point is a SQUID with the usual "loop"-geometry, consisting of one Josephson junction in each of the two parallel "arms" of the loop. The phase across the junctions in the left (index "l") and right (index "r") arm are thus both governed by an equation of the form (5.37). The difference between the two phases due to the vector potential of the enclosed magnetic flux is governed by the "flux quantization" relation

$$
\varphi_{l}-\varphi_{r}=2 \pi \Phi_{\text {tot }} / \Phi_{0}
$$

The enclosed flux $\Phi_{\text {tot }}$ is divided between an externally applied magnetic flux $\Phi$ and the flux from the circulating current in the loop, yielding 436]

$$
\Phi_{\text {tot }}=\Phi-\left[L_{l} I_{l}-L_{r} I_{r}\right]
$$

where $L_{l, r}$ are the inductances of the two junctions. Under the simplifying assumptions that

$$
C_{l}=C_{r}=: C \quad, \quad R_{l}=R_{r}=: R
$$


and with the definitions

$$
\begin{aligned}
I_{c}:=\frac{I_{c, l}+I_{c, r}}{2}, \quad \alpha_{I}:=\frac{I_{c, l}-I_{c, r}}{I_{c, l}+I_{c, r}} \\
\bar{L}:=\frac{L_{l}+L_{r}}{2}, \quad \alpha_{L}:=\frac{L_{l}-L_{r}}{L_{l}+L_{r}} \\
\varphi:=\frac{\varphi_{l}+\varphi_{r}}{2}, \quad \psi:=\frac{\varphi_{l}-\varphi_{r}}{2}
\end{aligned}
$$

it follows by adding and subtracting the two equations of the form (5.37) with indices "l" and "r" that 182, 183

$$
\begin{gathered}
\frac{\Phi_{0} C}{2 \pi} \ddot{\varphi}(t)+\frac{\Phi_{0}}{2 \pi R} \dot{\varphi}(t)=-\frac{\partial V(\varphi(t), \psi(t), t)}{\partial \varphi}+\frac{I_{\mathrm{tot}}(t)}{2}+\xi_{1}(t) \\
\frac{\Phi_{0} C}{2 \pi} \ddot{\psi}(t)+\frac{\Phi_{0}}{2 \pi R} \dot{\psi}(t)=-\frac{\partial V(\varphi(t), \psi(t), t)}{\partial \psi}+\xi_{2}(t) \\
V(\varphi, \psi, t):=-I_{c}\left[\cos \varphi \cos \psi-\alpha_{I} \sin \varphi \sin \psi\right] \\
+\frac{\pi}{4 \Phi_{0} \bar{L}}\left[\psi \frac{\Phi_{0}}{\pi}-\Phi+\bar{L} \alpha_{L} I_{\mathrm{tot}}(t)\right]^{2} .
\end{gathered}
$$

Here, $I_{\text {tot }}(t):=I_{l}(t)+I_{r}(t)$ is the total electrical current flowing through the SQUID and $\xi(t)(i=1,2)$ are two unbiased Gaussian white noises with correlation

$$
\left\langle\xi_{i}(t) \xi_{j}(s)\right\rangle=\frac{k_{B} T}{R} \delta_{i j} \delta(t-s) .
$$

Finally, the time averaged voltage $U$ across the loop is again given by (5.41).

Our first observation is that for $\bar{L} \ll \Phi_{0} /\left(I_{c}+I_{\text {tot }}(t)\right)$ it follows from (5.50) that $\psi \simeq \pi \Phi / \Phi_{0}$ and we are left with an effective one-dimensional problem (5.48). The same type of approximation has been made in the derivation of (5.38)-(5.40). In any case, the potential $(5.50)$ is periodic in the variable $\varphi$, while the $\psi$-dependence is confined by the quadratic term on the right hand side. On condition that $\Phi$ is not a multiple of $\Phi_{0} / 2$ and that either $\alpha_{I} \neq 0$ or $\alpha_{L} \neq 0$, the potential (5.50) is neither inversion symmetric under $(\varphi, \psi) \mapsto(-\varphi, \psi)$ nor $(\varphi, \psi) \mapsto(-\varphi,-\psi)$, thus a ratchet effect is theoretically predicted and has been experimentally observed [182, 183. Especially, a non-vanishing externally applied magnetic field is necessary, since otherwise $\Phi=0$.

Given that the above conditions $\left(2 \Phi / \Phi_{0}\right.$ not an integer and $\alpha_{I} \neq 0$ or $\left.\alpha_{L} \neq 0\right)$ are fulfilled, it is instructive to rewrite (5.48)-(5.50) in the form

$$
\begin{aligned}
& \frac{\Phi_{0} C}{2 \pi} \ddot{\varphi}(t)+\frac{\Phi_{0}}{2 \pi R} \dot{\varphi}(t)=-\frac{\partial \tilde{V}(\varphi(t), \psi(t))}{\partial \varphi}+\frac{I_{\text {tot }}(t)}{2}+\xi_{1}(t) \\
& \frac{\Phi_{0} C}{2 \pi} \ddot{\psi}(t)+\frac{\Phi_{0}}{2 \pi R} \dot{\psi}(t)=-\frac{\partial \tilde{V}(\varphi(t), \psi(t))}{\partial \psi}-\frac{\alpha_{L} I_{\text {tot }}(t)}{2}+\xi_{2}(t) \\
& \tilde{V}(\varphi, \psi):=-I_{c}\left[\cos \varphi \cos \psi-\alpha_{I} \sin \varphi \sin \psi\right]+\frac{\Phi_{0}}{4 \pi \bar{L}}\left[\psi-\frac{\pi \Phi}{\Phi_{0}}\right]^{2} .
\end{aligned}
$$

In other words, a two-dimensional rocking ratchet scheme is recovered, with a "rocking force" which acts along the $\varphi$-direction if $\alpha_{L}=0$ and points into a more general direction in the $\varphi$ - $\psi$-plane if $\alpha_{L} \neq 0$.

Further studies on related Josephson ratchet systems are addressed in sections 5.3.3 and 9.1, see also section 5.2. 


\subsection{Giant enhancement of diffusion}

In this section we return to the overdamped, one-dimensional tilting ratchet scheme (5.1), however, with the effective diffusion coefficient (3.6) rather than the particle current being the quantity of our interest. To this end, it turns out that the asymmetry of the potential $V(x)$ in (5.1) is not essential, and we will therefore focus on the simplest case of a symmetric, periodic potential $V(x)$.

In contrast to the investigation of directed transport in terms of $\langle\dot{x}\rangle$, studies of diffusive transport in periodic driven systems are still rather scarce. While the determination of the effective diffusion coefficient is, in general, technically more demanding (cf. section 3.1) its relevance e.g. for particle separation purposes may well be comparable to the schemes based on directed transport.

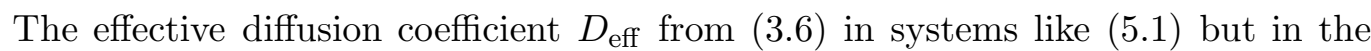
absence of an external driving $y(t)$ has been considered in 437 with the main result that $D_{\text {eff }}$ is for non-trivial potentials $V(x)$ always smaller than the bare diffusion coefficient (2.11). Diffusive separation of particles in the same system (5.1) but with a static tilt $y(t) \equiv F$ (cf. (2.34)) has been addressed in 438], demonstrating an improvement of one to two orders of magnitude in selectivity as compared with conventional continuous field free-flow electrophoresis methods, see also [117, 173, 174]. Asymptotic results for fast pulsating and fluctuating force ratchet schemes have been derived in [439] and [440], respectively. Here, we will focus on the case of a deterministic, timeperiodic perturbation $y(t)$ in (5.1), naturally arising in typical experimental settings. Related studies are 170, 391,441 443 and especially the work of Gang, Daffertshofer, and Haken [444]. Our present system is a conceptually simpler and more effective variation of the setup from [391, 444] which enables a controlled selective enhancement of diffusion that in principle can be made arbitrarily strong.

We focus on the simplest case of a symmetric sawtooth potential $V(x)$ with period $L$ and barrier height $V_{0}$ (figure 5.1 a) and a time-periodic driving force $y(t)$ with three states $y_{0}, 0$, and $-y_{0}$. As illustrated in figure 5.1 b, time-segments of length $t_{\mathrm{t}}$ with a constant tilt $y(t)= \pm y_{0}$ are separated by "waiting-periods" $t_{\mathrm{w}}$ with vanishing $y(t)$. Further, we henceforth restrict ourselves to weak thermal noise $\xi(t)$, i.e. $k_{B} T \ll V_{0}$.
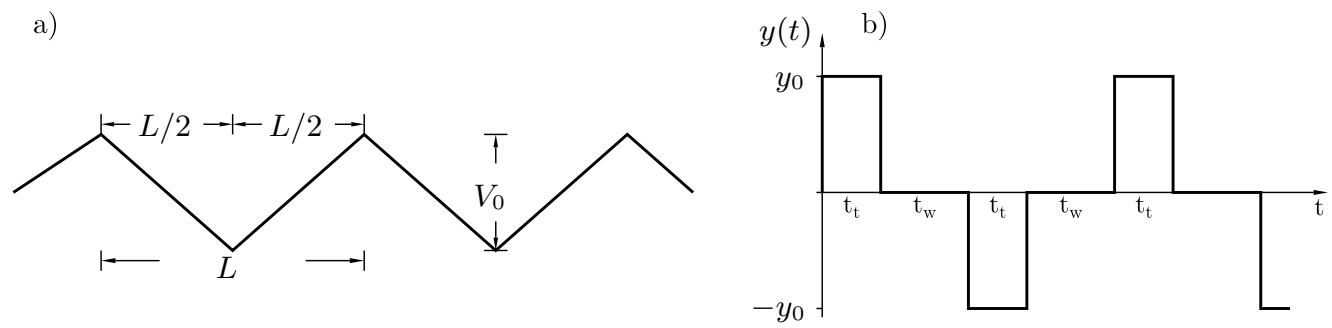

Figure 5.1: a) Symmetric sawtooth potential $V(x)$ with period $L$ and barrier height $V_{0}$. b) Time-periodic, piecewise constant driving force $y(t)$ with model-parameters $y_{0}$ ("tilt"), $t_{\mathrm{t}}$ ("tilting-time"), and $t_{\mathrm{w}}$ ("waiting-period").

We assume that $y_{0}>2 V_{0} / L$ and that the initial particle distribution at time $t=0$ consists of a very narrow peak at a minimum of the potential $V(x)$, say at $x=0$. As long as $t \leq t_{\mathrm{t}}$ we have $y(t) \equiv y_{0}$, so the peak moves to the right under the action of the deterministic forces and also broadens slightly due to the weak thermal noise in 
(5.1). The deterministic time $t_{n}$ at which the peak crosses the $n$-th maximum of $V(x)$ at $x=(n-1 / 2) L$ while $y(t)=y_{0}$ is acting, can be readily figured out explicitly [228]. If now $t_{\mathrm{t}}$ just matches one of those times $t_{n}$, then the original single peak is split into two equal parts and if the subsequent "waiting-interval" $t_{\mathrm{w}}$ with $y(t) \equiv 0$ is sufficiently long the two parts will proceed towards the respective nearest minimum of $V(x)$ at $x=(n-1) L$ and $x=n L$. The result consists in two very sharp peaks after half a period $t=t_{\mathrm{t}}+t_{\mathrm{w}}$ of the driving force $y(t)$. Similarly, after a full period $\tau:=2\left(t_{\mathrm{t}}+t_{\mathrm{w}}\right)$ one obtains three narrow peaks at $x=-L, 0, L$ with weights $1 / 4,1 / 2,1 / 4$, respectively. For the variance $\left\langle x^{2}(t)\right\rangle-\langle x(t)\rangle^{2}$ one thus obtains the result $L^{2} / 2$. In the same way one sees that after $n$ periods the variance amounts to $n L^{2} / 2$, yielding for the effective diffusion coefficient (3.56) the expression

$$
D_{\text {eff }}=L^{2} / 8\left(t_{\mathrm{t}}+t_{\mathrm{w}}\right) .
$$

In the case that $t_{\mathrm{t}}$ does not match any of the times $t_{n}$, the initial single peak is split after half a period $t=t_{\mathrm{t}}+t_{\mathrm{w}}$ into two peaks with unequal weights. If $t_{\mathrm{t}}$ is sufficiently different from any $t_{n}$ and the thermal fluctuations are sufficiently weak, one of those two peaks has negligible weight. Consequently, after a full period almost all particles will return to $x=0$. The effective diffusion coefficient $D_{\text {eff }}$ is therefore very small, in particular much smaller than for free thermal diffusion (2.11).
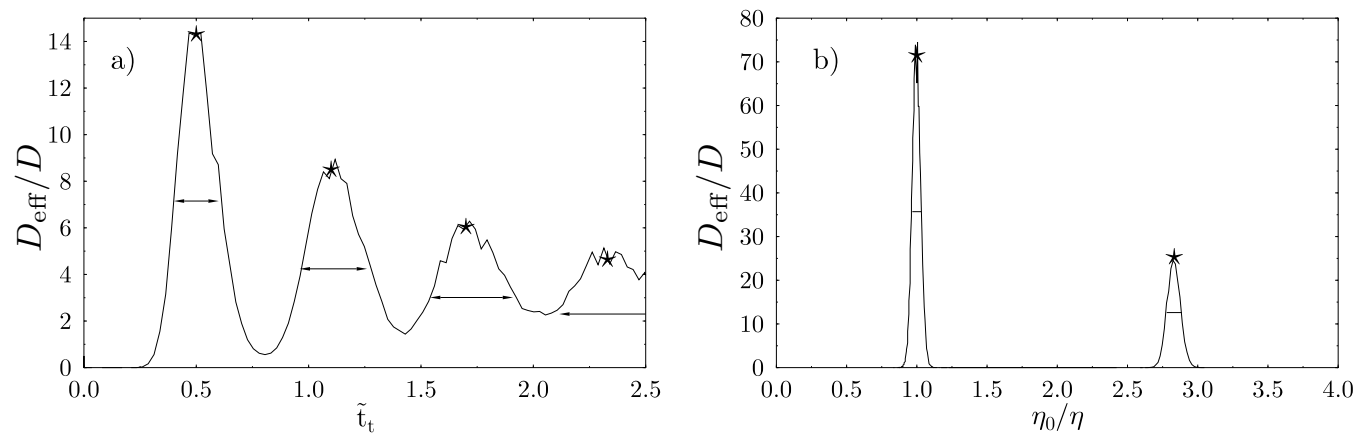

Figure 5.2: a) Effective diffusion coefficient (3.56) in units of the "bare" $D$ from (2.11) versus scaled "tilting-time" $\tilde{t}_{\mathrm{t}}:=t_{\mathrm{t}} V_{0} / \eta L^{2}$ from numerical simulations of the stochastic dynamics (5.1). The wiggles reflect the statistical uncertainty due to the finite though extensive number of realizations. The relevant dimensionless parameters in figure 5.1 are $k_{B} T / V_{0}=0.01, t_{\mathrm{w}} V_{0} / \eta L^{2}=0.375$, and $y_{0} L / V_{0}=3$. Theoretical predictions for the height of the peaks from (5.55) are indicated by stars. In addition, the theoretical estimate for the peak-widths at half height from 228] are indicated by arrows. b) Effective diffusion coefficient versus friction coefficient $\eta$ from simulations of (5.1) with $k_{B} T / V_{0}=0.005$ and $y_{0} L / V_{0}=22$. The times $t_{\mathrm{t}}$ and $t_{\mathrm{w}}=t_{\mathrm{t}}$ are both kept at fixed values and also define $\eta_{0}$ via $\eta_{0}=\left(2 L y_{0}-4 V_{0}\right) / L^{2} t_{\mathrm{t}}$. Theoretical predictions are indicated analogous to a).

An example of the effective diffusion coefficient $D_{\text {eff }}$ as a function of $t_{\mathrm{t}}$ is depicted in figure 5.2a. As usual (cf. section 3.6) such a multi-peak-structure of $D_{\text {eff }}$ is not only expected upon variation of $t_{\mathrm{t}}$ but also by keeping $t_{\mathrm{t}}$ fixed and varying for instance the friction coefficient $\eta$, corresponding to the situation that different types of particles are moving in the same rocked periodic potential. As figure 5.2 $\mathrm{b}$ demonstrates, the dynamics (5.1) can indeed act as an extremely selective device for separating different 
types of particles by controlled, giant enhancement of diffusion. Closer inspection shows [228] that the peaks in the effective diffusion coefficient $D_{\text {eff }}$ can in fact be made arbitrarily narrow and high by decreasing the temperature or increasing $V_{0}$ at fixed $T$ while at the same time keeping $y_{0} L / V_{0}$ large. Similarly as for the friction coefficient $\eta$, particles can also be separated, e.g., according to their electrical charge since this implies different values of the "coupling-parameters" $V_{0}$ and $y_{0}$.

All these findings are obviously robust against various modifications of the model as long as one maintains periodicity in space and time and sufficiently long "waitingperiods" $t_{\mathrm{t}}$ with $y(t) \equiv 0$ between subsequent "tilting-times" with non-vanishing $y(t)$. A practical realization of such a particle separation device should be rather straightforward.

\subsection{Asymmetrically tilting ratchets}

In this section we consider the ratchet model dynamics (5.1) with a symmetric, $L$ periodic potential $V(x)$ in combination with a driving $y(t)$ of broken symmetry, either periodic or stochastic.

If the characteristic time scale of the driving $y(t)$ is very large, the adiabatic approximation (5.2) for the periodic and (5.9) for the stochastic case can be applied. Exploiting the symmetry of $V(x)$, a straightforward calculation confirms the expected property that $v(y)$ in (5.3) is an odd function of its argument. In general, the contributions of $y$ and $-y$ in (5.2) or (5.9) will not cancel each other and hence $\langle\dot{x}\rangle$ will generically be different from zero. However, even though $y(t)$ is asymmetric, prominent examples exists for which the contributions of $y$ and $-y$ do cancel each other, namely those respecting supersymmetry $(3.40)$. Examples are a periodic driving $y(t)$ of the form (3.47) with $\gamma_{1} \neq 0$ and $\gamma_{2} \neq 0$ or the example depicted in figure 3.2. In this case, $\langle\dot{x}\rangle \rightarrow 0$ as the characteristic time scale of $y(t)$ tends to infinity, which is a quite exceptional feature within the class of tilting ratchets. Since for fast driving the current approaches zero as well, a qualitative behavior which in fact is reminiscent of a pulsating ratchet arises. It is worth emphasizing, since it may appear counterintuitive at first glance, that a symmetric, but not supersymmetric potential $V(x)$ (e.g. in (3.19) with $a_{1} \neq 0$ and $\left.a_{2} \neq 0\right)$ combined with a supersymmetric but not symmetric $y(t)$ (e.g. in figure 3.2) generically does lead to a ratchet effect ${ }^{20}\langle\dot{x}\rangle \neq 0$, see also figure 3.3. If moreover finite inertia effects $m \ddot{x}(t)$ are included on the right hand side of (5.1) then supersymmetry does no longer prohibit a current and thus even a pure sinusoidal potential $V(x)$ may be chosen.

\subsubsection{Periodic driving}

The case of slow periodic driving is covered by the adiabatic approximation (5.2). In the opposite case of a very small period $\mathcal{T}$, one finds along the same line of reasoning

\footnotetext{
${ }^{20}$ To dissolve any remaining doubts, we have verified this fact by numerical simulations. A similar prediction has been put forward previously in [445, 446] without, however, recognizing the subtleties of supersymmetry in this context.
} 
as in section 5.3.1 the leading order asymptotics 389

$$
\begin{aligned}
\langle\dot{x}\rangle & =\frac{\mathcal{T}^{4} L\left[Y_{-} \int_{0}^{L} d x\left[V^{\prime \prime \prime}(x)\right]^{2}+Y_{+} \int_{0}^{L} d x\left[V^{\prime \prime}(x)\right]^{3} / 2 k_{B} T\right]}{4 \eta^{5} \int_{0}^{L} d x e^{V(x) / k_{B} T} \int_{0}^{L} d x e^{-V(x) / k_{B} T}} \\
Y_{ \pm} & :=\int_{0}^{1} d h\left[\hat{y}_{0}(h) \pm 2 \hat{y}_{2}(h)\right]\left[\hat{y}_{2}(h)\right]^{2}
\end{aligned}
$$

where $\hat{y}_{0}(h)$ and $\hat{y}_{2}(h)$ are defined in (5.26) and (5.27). Here we have exploited the symmetry of $V(x)$. In the completely general case, the asymptotic current $\langle\dot{x}\rangle$ is given by the sum of the contributions in (5.31) and (5.56).

We notice that if the potential $V(x)$ is not only symmetric but also supersymmetric then $\int_{0}^{L} d x\left[V^{\prime \prime}(x)\right]^{3}=0$ and thus the sign of the current in (5.56) is dictated solely by that of $Y_{-}$. On the other hand, for a supersymmetric driving $y(t)$, both coefficients $Y_{ \pm}$ in (5.57) vanish, that is, $\langle\dot{x}\rangle$ approaches zero even faster than $\mathcal{T}^{4}$ as $\mathcal{T} \rightarrow 0$.

The possibility that a directed current, or, equivalently, a finite voltage under open circuit conditions, may emerge in a symmetric periodic structure when driven by unbiased, asymmetric microwave signals of the form

$$
y(t)=\gamma_{1} \cos (2 \pi t / \mathcal{T})+\gamma_{2} \cos (4 \pi t / \mathcal{T}+\Phi)
$$

has been reported for the first time in the experimental work by Seeger and Maurer [30]. From the traditional viewpoint of response theory in this context, the basic mechanism responsible for producing a dc-output by an unbiased ac-input (5.58) then amounts to the so-called harmonic mixing of the two microwaves of frequencies $2 \pi / \mathcal{T}$ and $4 \pi / \mathcal{T}$ in the nonlinear response regime. The electrical transport in such quasi-onedimensional conductors is usually described in terms of pinned charge density waves, which in turn are modeled phenomenologically as an overdamped Brownian particle in a symmetric, periodic "pinning" potential [31, 218 220]. The particle couples to the externally applied field (5.58) via an effective charge, i.e. we recover exactly the asymmetrically tilting ratchet model (5.1). We remark that both in the experimental work [30] and in the subsequent theoretical studies [31, 218 220] no emphasize is put on the fact of generating a dc-output by means of an unbiased ac-input per se, and in this sense the ratchet effect has been observed only implicitly. Also worth mentioning is that the "pinning"-potential $V(x)$ is usually assumed to be of sinusoidal shape and thus respects supersymmetry. Since the driving (5.58) becomes supersymmetric for $\Phi=\pi / 2$, the current $\langle\dot{x}\rangle$ will exactly vanish at this point [31, 218, 219]. This feature does no longer arise for symmetric but not supersymmetric potentials $V(x)$ or if finite inertia effects [220] become relevant, see also [215, 400].

In the context of current generation by photovoltaic effects (cf. at the end of section 5.2) very closely related theoretical and experimental investigations are due to 384 388. The same basic idea to produce a directed current by means of the asymmetric tilting ratchet scheme has also been exploited experimentally in a process called zero-integrated field gel electrophoresis 2 which uses unbiased pulsed electric fields to separate chromosomal DNA 423 427,447 449.

The ratchet effect in a periodically driven, asymmetrically tilting ratchet has been independently re-discovered in [39]. Moreover, the complex structure of the current

\footnotetext{
${ }^{21}$ The gel network in which the DNA moves does not exhibit the usual spatial periodicity but rather acts as a random potential (due to basically static obstacles) in three dimensions.
} 
$\langle\dot{x}\rangle$ at $T=0$, featuring plateaux and Devil's staircases, similarly as for the rocking ratchet system in section 5.3.2, has been demonstrated for especially simple examples of asymmetrically tilting ratchet models in [39]. Further variations and extensions of such theoretical models, the details of which go beyond our present scope, can be found in 215, 259, 359, 423, 425 427, 447, 450 453]. For Hamiltonian (finite inertia, vanishing dissipation and thermal noise) and quantum mechanical asymmetrically tilting ratchet systems we refer to section 5.4 and to sections 8.3 and 8.4 , respectively.

\subsubsection{Stochastic, chaotic, and quasiperiodic driving}

The generation of directed transport in symmetric, periodic potentials $V(x)$ by an asymmetric stochastic driving $y(t)$ of zero average in (5.1) has been for the first time exemplified in [330 333] for the case of Poissonian white shot noise ${ }^{22}$, see also [179, 454]. At zero thermal noise $(T=0)$, a closed analytical solution is available [330, 332], while for $T>0$ one has to recourse to asymptotic expansions, piecewise linear potentials, or numerical evaluations 331, 333]. Besides the fact of a white-noise induced directed transport in symmetric potentials per se, the most remarkable finding is that the current always points into the same direction as the $\delta$-spikes of the asymmetric shot noise for any periodic potential (symmetric or not, but different from the trivial case $\left.V^{\prime}(x) \equiv 0\right)$. We are thus facing one of the rare cases for which our procedure of tailoring current inversions (see section 3.6) cannot be applied unless an additional systematic bias $F$ is included in (5.1). Leaving aside minor differences in the $\delta$-spikes statistics (cf. footnote 12) the basic reason for this unidirectionality can be readily understood by the mapping onto an improper traveling potential ratchet scheme according to (4.31), (4.32) and our discussion of the corresponding current (4.33), (4.35), (4.38).

The generic occurrence of a ratchet effect whenever $y(t)$ breaks the symmetry (3.18) has been pointed out in [356] and exemplified by means of an asymmetric two-state noise $y(t)$ in the adiabatic limit, cf. (5.9). Similar conclusions have been reached in [179, 180. In the case of an asymmetric dichotomous noise $y(t)$ and without thermal fluctuations $(T=0)$ in (5.1), the exact analytical solution for arbitrary noise characteristics and potentials has been figured out and discussed from different viewpoints in [193, 358, 367, 455, 456]. Similarly as in (5.17), the above mentioned shot noise model [330,332] is recovered as a special limit [329] from this analytical solution for dichotomous noise. Approximations and analytically soluble particular cases in the presence of a finite amount of thermal noise $(T>0)$ have been elaborated in [291, 368]. Regarding the asymptotics of fast asymmetric tilting we remark that the expression (5.18) vanishes for symmetric potentials $V(x)$, hence a significantly different structure of the leading order behavior is expected (compare also the corresponding results (5.31) and (5.56) for periodic $y(t))$. For asymmetric dichotomous noise such an asymptotics has been derived in [368] within a constant intensity scaling scheme, while for constant variance scaling, as we mainly consider it in our present review, such an asymptotics has not yet been worked out.

Turning to applications, it has been argued in [356] that the absence of a priori symmetry reasons and thus the appearance of an asymmetric noise $y(t)$ should be a rather common situation in many systems far from equilibrium, especially in biochem-

\footnotetext{
${ }^{22}$ The specific shot noise considered in 330 333] is of the form 4.43)-(4.44) but with the weights $n_{i}$ in (4.44) not being integers but rather exponentially distributed, positive random numbers, see also $5.13), 5.15$.
} 
ical contexts involving catalytic cycling (cf. section 4.6 and chapter 7). Specifically, if $y(t)$ represents a source of unbiased nonequilibrium current fluctuations then an asymmetrically tilting ratchet scheme can be readily realized by means of a Josephson junction [358,455, 456], see (5.37). A concrete such source of current fluctuations has been pointed out in [193]. Namely, an asymmetric dichotomous noise may arise intrinsically in point contact devices with a defect which tunnels incoherently between two states [132 138. A modified Josephson junction system with an asymetric total noise composed of two correlated symmetric noise sources has been proposed in 457, 458, see also 459461 .

It is well-known 402 404,462 464 that in many situations, a low dimensional dynamical system exhibiting deterministic chaos can induce similar effects as a veritable random noise 3 . In the present case of the asymmetrically tilting ratchet scheme, the emergence of directed transport (ratchet effect) when the driving $y(t)$ is generated by a low dimensional chaotic dynamics has been demonstrated in [36], see also [179, 180,465].

Another interesting intermediate between a stochastic and a periodic driving is represented by the case of a quasiperiodic driving $y(t)$, bringing along the possibly of a strange nonchaotic attractor [466]. Asymmetrically tilting ratchets of this type have been studied in [181.

\footnotetext{
${ }^{23}$ In fact, we may consider a noise (stochastic process) as generated by a chaotic deterministic dynamics in the limit of infinitely many dimensions. The close similarity between deterministic chaos and noise is also exploited in any numerical pseudo-random number generator.
} 


\section{Chapter 6}

\section{Sundry Extensions}

In this chapter we address various significant modifications and extensions of the pulsating and tilting ratchet schemes from chapters 4 and 5 as well as an additional important observable in the context of Brownian motors, namely their efficiency. Remarkably, while most of those generalizations are conceptually very different from a pulsating or tilting ratchet in the original sense, an approximate or even exact mathematical equivalence can be established in several cases. In other cases, both the physics and the mathematics are fundamentally different.

\subsection{Seebeck ratchets}

In this section we consider periodic systems under the influence of thermal fluctuations, the intensity of which exhibits a spatial variation with the same periodicity as the relevant potential, while no other non-equilibrium perturbations are acting.

In a closed circuit composed of two dissimilar conductors (or two dissimilarly doped semiconductors) a permanent electric current arises when their junctions are kept at different temperatures [467]. This constitutes a thermoelectric circuit that converts thermal energy into electrical energy. The effect has been discovered in 1822 by Seebeck and has been exploited, e.g., to provide electrical power for satellites. In essence, the Seebeck effect has the following microscopic origin: Due to the different Fermi-levels prevailing in each of the conductors, a kind of effective potential ramp for the electrons arises at the junction円. Moving along the circuit in a definite direction, the electrons will encounter at one junction an increasing potential ramp and at the other junction a decreasing counterpart. When looping in the opposite direction, the roles of the ramps is exchanged. While sliding down a decreasing ramp is "for free", climbing up an increasing ramp requires thermal activation. Therefore, if one junction is kept at a higher temperature than the other, the looping of electrons in one direction is more likely than in the other.

Expanding the circular motion through the closed circuit to the real axis yields a periodic effective potential $V(x)$ and a periodic temperature profile $T(x)$. Both have the same spatial period and each of them is typically symmetric under spatial inversion. The spatial symmetry of the system is broken in that the two periodic functions $V(x)$

\footnotetext{
${ }^{1}$ Within this very elementary picture we neglect electron-electron interaction effects in the form of screening by inhomogeneous charge densities around these potential ramps 468 .
} 
and $T(x)$ are out of phased. The simplest model for the electron motion consists in an overdamped dynamics like in (4.17) with

$$
g(x)=\left[k_{B} T(x) / \eta\right]^{1 / 2} .
$$

This model has been studied by Büttiker [33] and independently by van Kampen [469], and has been further discussed by Landauer 470]. Later, similar models, either derived from a microscopic description of the environment in terms of harmonic oscillators (cf. section 8.1), or based on a phenomenological approach have been considered in [190, 191, 471, 472] and [295, 296, 473, 474], respectively, see also section 6.4.

Though the physical systems behind this Seebeck ratchet model and the one in (4.17) are quite different, the mathematics is practically the same and in this sense the Seebeck ratchet is closely related to a fluctuating potential ratchet [51]. One difference is that in one case the potential $V(x)$ is asymmetric and the fluctuations of this potential of course "in phase" with the "unperturbed" (average) potential, while in the other case the symmetry is broken due to a phase shift between $V(x)$ and $T(x)$. A second possible difference is that after the white noise limit $\tau \rightarrow 0$ in (4.19) the adequate treatment of the multiplicative noise in (4.17) may not always be in the sense of Stratonovich. For instance, if the dynamics (4.17) arises as limiting case with negligible inertia effects (white noise limit $\tau \rightarrow 0$ in $(4.19)$ before the limit of vanishing mass) then [294, 471, 472, 475, 476] a white noise $\xi(t)$ in the sense of Ito [63, 99] arises in (4.17). As a consequence, the second summand in (4.25) takes the modified form $\partial g^{2}(x) / \partial x$ and the integrand in (4.26) acquires an extra factor $g(y) / g(x)$. A still different treatment of the thermal noise $\xi(t)$ in (4.17) may be necessary in physical contexts without an inertia term right from the beginning, see 469, 470, 477] and further references therein. Independent of these details, the main conclusion is that $\langle\dot{x}\rangle \neq 0$ if and only if

$$
\int_{0}^{L} \frac{V^{\prime}(x)}{T(x)} d x \neq 0
$$

provided that both, $V(x)$ and $T(x)$ are $L$-periodic 3 . One readily verifies that the two "systematic" conditions implying $\langle\dot{x}\rangle=0$ are indeed the symmetry and supersymmetry criteria from (3.49) and (3.50), respectively.

Though the Seebeck ratchet thus exhibits striking similarities with a fluctuating potential ratchet, the equivalence is not exact. However, such an exact equivalence can be readily established with respect to the more general class of pulsating ratchet models by choosing $\xi(t) \equiv 0$ and

$$
V^{\prime}(x, f(t))=V^{\prime}(x)+\sqrt{2 \eta k_{B} T(x)} f(t)
$$

\footnotetext{
${ }^{2} \mathrm{~A}$ ratchet effect also arises for asymmetric $V(x)$ and/or $T(x)$ in phase, however, typically in a quite different physical context, see section 4.3.2.

${ }^{3}$ Similarly as in equation 4.27 ), the sign of the current $\langle\dot{x}\rangle$ is found to be opposite to the sign of the intergal on the left hand side of (6.2). Therefore, a current inversions upon variation, e.g., of $\eta$ is not possible in this model.

${ }^{4}$ Strictly speaking, (6.3) does still not respect the $L$-periodicity $(3.3)$. To remedy this flaw, one has to multiply the square-root in $(6.3)$ by a factor $\chi(x)$, defined as $\chi(x):=1$ for $x \in\left[0, x_{0}\right), \chi(x):=$ -1 for $x \in\left[x_{0}, L\right)$, and $\chi(x+L):=\chi(x)$. The reference position $x_{0}$ is then chosen such that $\int_{0}^{L} \chi(x)[T(x)]^{1 / 2} d x=0$ with the result that 3.3 is indeed satisfied. Note that this extra factor $\chi(x)$ in (6.3) does not affect the stochastic dynamics 3.1) in any noticeable way.
} 
with $f(t)$ being a $\delta$-correlated Gaussian noise. The basic physical picture underlying this mathematical equivalence is rather simple: Thermal fluctuations with a spatially periodic variation of their strength (temperature) may equivalently be viewed as (very fast) potential fluctuations (cf. section 4.3.2). We furthermore remark that by first applying the transformation (6.3) to a pulsating ratchet, and then considering the symmetry and supersymmetry criteria (3.16) and (3.39) for such a pulsating ratchet model, one indeed recovers the corresponding original criteria for Seebeck ratchets in (3.49) and (3.50), respectively.

Besides the Seebeck effect itself, another application of the model may be the electron motion in a superlattice irradiated by light through a mask of the same period but shifted with respect to the superlattice [33]. In such a case, it may no longer be justified to neglect inertia effects in the stochastic dynamics (4.17). The so-called underdamped regime of such a dynamics, i.e. friction effects are weak in comparison to the inertia effects, has been analytically treated in 478] by generalizing the methods developed in 479.

There are several well-known phenomena which may in fact be considered a close relatives of the Seebeck effect and thus as further instances of the corresponding ratchet scheme: First, we may augment our closed circuit, composed of two differently doped semiconductors, by a piece of a metal wire. In other words, we are dealing with an electrical circuit that contains a semiconductor diode (n-p junction). Again, an electrical current results if the diode is kept at a temperature different from the rest of the circuit (thermogenerator), see also sections 2.9 and 8.4 . Second, the same device can also act as a photodiode or photoelement by exposing the n-p junction to a source of light. Especially, in the case of black-body irradiation, one basically recovers the previous situation with two simultaneous heat baths at different temperatures. Third, one may replace the semiconductor diode by a tube diode. In this context, the two above mentioned ways of generating an electrical current are then closely related to the so-called Richardson-effect and photoeffect, respectively.

\subsection{Feynman ratchets}

Throughout the discussion of Smoluchowski and Feynman's Gedankenexperiment in section 2.1.1 we have assumed that the entire gadget in figure 2.1 is surrounded by a gas at thermal equilibrium. In his lectures [2], Feynman also goes one step further in considering the case that the gas around the paddles is in a box at temperature $T_{1}$, while the ratchet and pawl are in contact with a different bath (e.g. another gas in a box) at temperature $T_{2} \neq T_{1}$, see figure 6.1.

While Feynman's discussion [2] focuses on a thermodynamic analysis of this nonequilibrium system and apparently contains a misconception [110, 111, 480, 481], here we concentrate on its microscopic modeling in terms of a stochastic process. Our first observation is that there are essentially two relevant (slow) collective coordinates: One is an angle, which characterizes the relative position of the pawl and an arbitrary reference point on the circumference of the ratchet in figure 6.1 and which we will henceforth consider as expanded to the entire real axis and denoted as $x(t)$. As we have seen in section 2.1.1, the possibility that the pawl spontaneously (due to thermal fluctuations) lifts itself up so that the ratchet can freely rotate underneath, is a crucial feature of the system. Therefore, another relevant collective coordinate is the "height" $h(t)$ of the pawl, i.e. its position in the direction perpendicular to $x$ (the "radial" 


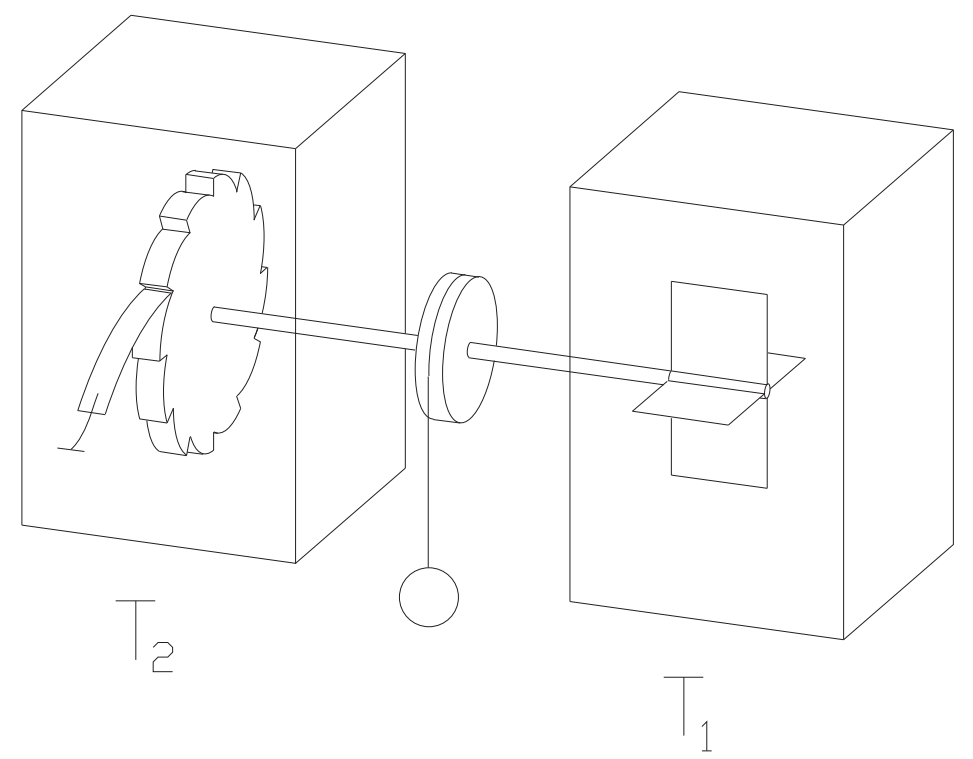

Figure 6.1: $\quad$ Same as figure 2.1 but with the ratchet and pawl kept at a different temperature than the paddles and its surrounding gas.

direction in figure 6.1).

The next modeling step consists in taking into account the thermal environment of the paddles, governing the state variable $x(t)$, and the second heat bath, governing the dynamics $h(t)$ of the pawl. A realistic description both of the impacts of the gas molecules on the paddles (e.g. by means of a Boltzmann-equation [215]) and of the thermal fluctuations of the pawl on a microscoping footing is very involved. Along the general spirit of section 2.1, a phenomenological modeling is the only realistically practicable modeling approach. In a first approximation 111, 480, these environmental effects may be modeled by an overdamped dynamics for both $x(t)$ and $h(t)$, i.e.

$$
\begin{aligned}
& \eta_{1} \dot{x}(t)=-\frac{\partial V(x(t), h(t))}{\partial x}+\xi_{1}(t) \\
& \eta_{2} \dot{h}(t)=-\frac{\partial V(x(t), h(t))}{\partial h}+\xi_{2}(t)
\end{aligned}
$$

with two independent white (thermal) Gaussian noises

$$
\left\langle\xi_{i}(t) \xi_{j}(s)\right\rangle=2 \eta_{i} k_{B} T_{i} \delta_{i j} \delta(t-s)
$$

at temperatures $T_{1}$ and $T_{2}$, respectively [110]. The interaction between $x(t)$ and $h(t)$ arises through the common potential $V(x, h)$ which incorporates the fact that the pawl is (weakly) pressed against the ratchet (e.g. by a spring or due to its own elasticity) and the constraint that the pawl cannot penetrate the ratchet. The latter, non-holonomous constraint can be included by appropriate "potential walls" into $V(x, h)$. An explicit example [111] is

$$
V(x, h)=\kappa h+\frac{\mu}{h-H(x)}
$$


where $\kappa$ is the "spring constant" of the pawl, $H(x)$ is the geometrical profile of the ratchet, and $\mu$ is a parameter characterizing the "steepness" of the potential walls which account for the constraint $h>H(x)$.

Note that (6.4) seems in fact to perfectly fit into the general framework of a fluctuating potential ratchet scheme (4.1). However, it actually goes somewhat beyond this scheme in that our usual assumption of the "potential fluctuations" $h(t)$ being independent of the system $x(t)$ is no loner respected, there is a "back-coupling" in (6.5).

In spite of the various so far made approximations, the model is still only tractable by means of numerical simulations. Detailed quantitative results of such simulations can be found in 111, 480. Here, we proceed with the additional approximation that the pawl remains permanently in contact with the ratchet, i.e. the constraint $h>H(x)$ is replaced by $h=H(x)$. Physical realizations of such a modified system with a fixed, one-dimensional "track" $(x, H(x))$ of the pawl can be readily figured out. Moreover, it is clear that in those regions of the track with a small slope $H^{\prime}(x)$, the noise acting on $x(t)$ dominates, while the noise acting on $h(t)$ dominates for large slopes $H^{\prime}(x)$. In other words, an effective one-dimensional ratchet dynamics with a state dependent effective temperature $T(x)$ is recovered [111,481 483]: The Feynman ratchet can be approximately reduced to a Seebeck ratchet model.

The main results of such a simplified one-dimensional description are qualitatively the same as for the more complicated two-dimensional original model (6.4)-(6.7) 111, 484: If the paddles experience a higher temperature than the pawl $\left(T_{1}>T_{2}\right)$ then the rotation is in the direction naively expected already in figure 2.1. Remarkably, for $T_{1}<T_{2}$ the direction is inverted, i.e. the pawl preferably climbs up the steep slope of the ratchet profile $H(x)$.

Experimental realizations of the above Feynman ratchet and pawl gadget are not known. In order that thermal fluctuations will play any significant role, such an experiment has to be carried out on a very small scale. Quantitative estimates in 111 indicate that the necessary temperature differences in order to achieve an appreciable ratchet effect are probably not experimentally feasible. However, modified two-dimensional settings of the general form (6.4)-(6.7), e.g. with $\xi_{2}(t)$ consiting of a thermal noise at the same temperature as $\xi_{1}(t)$ and a superimposed external driving, may well be experimentally realizable, see section 5.5. Finally, a Feynman ratchet-type model for a molecular motor (cf. chapter 7) has been proposed in [5, 6], though this model was later proven unrealistic by more detailed quantitative considerations 142, 143].

\subsection{Temperature ratchets}

The properties and possible applications of the temperature ratchet [118] with timeperiodic temperature variations (2.6), (2.47) have been discussed in detail in sections 2.6, 2.10, and 2.11. A modified model in which the temperature changes $T(t)$ are governed by a dichotomous random process (cf. equations (3.11)-(3.13)) has been studied in [126, 127, 485, 486]. The resulting, so-called composite noise $\xi(t)$ gives rise to a "minimal" ratchet model in (2.6) in the sense that $\xi(t)$ is a stationary, unbiased, white noise with correlation

$$
\langle\xi(t) \xi(s)\rangle=2 \eta k_{B} T\left(1+\sigma^{2}\right) \delta(t-s) .
$$


The noise is, however, not a thermal noise (e.g. it is not Gaussian distributed), thus the generic appearance of the ratchet effect is not in contradiction to the second law of thermodynamics 126, 127, 485, 486.

Next, we consider again the general case that $T(t)$ may be either a periodic function or a random process, satisfying $T(t) \geq 0$ for all $t$. Introducing the auxiliary time 118, 487

$$
\begin{aligned}
& \hat{t}(t):=\int_{0}^{t} d t T(t) / \bar{T} \\
& \bar{T}:=\lim _{t \rightarrow \infty} \frac{1}{t} \int_{0}^{t} d t T(t)
\end{aligned}
$$

it follows that the temperature ratchet dynamics (2.48) can be rewritten in terms of $y(\hat{t}):=x(t(\hat{t}))$ in the form

$$
\begin{aligned}
\eta \dot{y}(\hat{t}) & =-V^{\prime}(y(\hat{t}))[1+f(\hat{t})]+\bar{\xi}(\hat{t}) \\
f(\hat{t}) & :=\frac{d t(\hat{t})}{d \hat{t}}-1,
\end{aligned}
$$

where $t(\hat{t})$ is the inverse of (6.9) (which obviously exists) and where $\bar{\xi}(\hat{t})$ is a Gaussian white noise with correlation

$$
\langle\bar{\xi}(\hat{t}) \bar{\xi}(\hat{s})\rangle=2 \eta k_{B} \bar{T} \delta(\hat{t}-\hat{s})
$$

which is moreover statistically independent of $f(\hat{t})$. Exploiting (6.9), (6.10) one can furthermore show that $f(\hat{t})$ is unbiased.

In general, if $T(t)$ is a stochastic process then the relation between properties of $y(\hat{t})$ and $x(t)$ is not obvious, since the time-transformation (6.9) is different for each realization of $T(t)$. However, with respect to the steady state current we can infer from the self-averaging property (3.5) in combination with (6.9) that

$$
\langle\dot{y}\rangle=\langle\dot{x}\rangle .
$$

If $T(t)$ is a periodic function of $t$ then the very same conclusion follows immediately. In other words, from (4.11), (6.11), (6.14) we can conclude that, at least with respect to the particle current, the temperature ratchet (2.6), (2.47) is exactly equivalent to a fluctuating potential ratchet (4.11), independently of whether the time variations of $T(t)$ are given by a periodic function or a stochastic process. On the other hand, a fluctuating potential ratchet can be mapped onto a temperature ratchet, provided $f(t)>-1$ for all $t$ in (4.11). Especially, from the asymptotics (4.10) for fast stochastic potential fluctuations the corresponding result [126, 485,486] for a temperature ratchet is recovered. Likewise, from the prediction (2.58) for a periodically modulated temperature ratchet we can read off the asymptotics for ratchets with fast, periodically fluctuating potentials. For similar reasons, the qualitative analysis of the temperature ratchet for slow dichotomous temperature variations in figure 2.6 is practically the same as for the on-off ratchet scheme [34].

The basic physical picture behind this equivalence of a temperature ratchet and a fluctuating potential ratchet is as follows: Very loosely speaking one may mimic temperature modulations by potential modulations since, under many circumstances, 
it is mainly the ratio of potential and temperature which plays the dominant role in transport phenomena (cf. figure 2.6).

We finally recall that, apart from "accidental" cases, the "systematic" conditions implying $\langle\dot{x}\rangle=0$ are the symmetry and supersymmetry criteria from (3.51) and (3.52), respectively. Not surprisingly, these are practically the same as the corresponding criteria of symmetry $(3.16)$ and supersymmetry $(3.39)$ for a fluctuating potential ratchet $V(x, f(t))=V(x)[1+f(t)]$.

\subsection{Inhomogeneous, pulsating, and memory friction}

\subsubsection{A no-go theorem}

In the preceding sections we have discussed modifications of the SmoluchowskiFeynman ratchet model (2.6) with either a spatial or a temporal variation of the temperature $T$ in (2.5). In the generic case, a finite particle current $\langle\dot{x}\rangle$ results in such a model, as expected from Curie's principle. In the following, we discuss an apparently rather similar modification of the Smoluchowski-Feynman ratchet model (2.5), (2.6), namely spatial and/or temporal variations of the friction coefficient $\eta$, with the rather unexpected result that the average particle current in the steady state is always zero.

In the case of a non-constant friction coefficient $\eta(x, t)$, the overdamped limit $m \rightarrow$ 0 is a subtle issue 469,471, 472, 488, 489 and one better keeps a finite mass $m$ in (2.1) right away. The corresponding Fokker-Planck equation for the probability density $P=P(x, v, t)$ follows along the same line of reasoning as in section 2.2 and Appendix $\mathrm{B}$ with the result 99

$$
\frac{\partial}{\partial t} P=-v \frac{\partial}{\partial x} P+\frac{1}{m} \frac{\partial}{\partial v}\left\{V^{\prime}(x)+\eta(x, t) v+\frac{\eta(x, t) k_{B} T}{m} \frac{\partial}{\partial v}\right\} P
$$

where $v:=\dot{x}$. Going over to the reduced density $\hat{P}(x, v, t)$ (cf. (2.22)), which is periodic in $x$ but still satisfies (6.15), one readily verifies that the Boltzmann distribution

$$
\hat{P}^{\mathrm{st}}(x, v)=Z^{-1} \exp \left\{-\left[m v^{2} / 2+V(x)\right] / k_{B} T\right\}
$$

is a steady state solution (cf. (2.31)). Under the sufficient (but not necessary) condition that $\eta(x, t)>0$ for all $x$ and $t$ (and that $T>0$ ) this long time asymptotics can be proven to be furthermore unique [82, 83, 100, 108, 109]. The remarkable feature of the steady state distribution (6.16) is that the friction coefficient $\eta(x, t)$ does not appear at all. For $\eta=$ const. we are dealing with an equilibrium system and the second law of thermodynamics implies the result

$$
\langle\dot{x}\rangle=0
$$

for similar reasons as in section 2.1. Considering that (6.16) does not depend on the friction coefficient, it is quite plausible that the result (6.17) carries over to arbitrary $\eta(x, t)$. The same conclusion is corroborated 191, 471, 472, 474 by a more detailed calculation similarly as in sections 2.3 and 2.4 .

The basic physical reason behind the result (6.17) is that the model (2.1), (2.5) describes an equilibrium system for arbitrary $\eta(x, t)$ : In fact, we have noticed below equation (2.5) that the friction coefficient can also be considered as the coupling strength between the system and its thermal environment. In the absence of other 
perturbations, the model (2.1) thus continues to describe an equilibrium system even for a non-constant coupling $\eta(x, t)$. Since an equilibrium system reaches an equilibrium state in the long time limit, the second law of thermodynamics can be invoked and (6.17) follows. (Only the transient dynamics depends on the details of $\eta(x, t)$.) Thus, there is no contradiction to Curie's principle: The current-prohibiting symmetry, which is easily overlooked at first glance, is in fact once again the detailed balance symmetry.

Returning finally to the overdamped limit $m \rightarrow 0$, we only state here the outcome of a more rigorous analysis [469,471, 472, 488, 489], namely that this limit cannot be consistently carried out in the stochastic dynamics (2.1) itself but only on the level of the Fokker-Planck equation (6.15), with the result of a probability current in (2.17) of the form like in (2.21) with $\eta(x, t)$ in place of $\eta$. The conclusion (6.17) then follows along the same line of reasoning as in section 2.4.

\subsubsection{Inhomogeneous and pulsating friction}

The microscopic origin of a time-independent inhomogeneous friction mechanism $\eta(x)$ has been discussed in section 3.4.1, namely a broken translation invariance of the thermal environment with respect to the relevant (slow) state variable(s) of interest. Physical examples are the Brownian motion near geometrical confinements of the fluid due to deviations from Stokes friction [376, 474, 490 492], phase dependent dissipation in Josephson junctions due to the interference of pair and quasiparticle tunneling currents [493], generic chemical reactions [494, 495] (cf. section 3.4.1), and protein friction in molecular motors, see section 7.3. In the following, we restrict ourselves to the most important case that $\eta(x)$ is strictly positive and exhibits the same periodicity $L$ as the potential $V(x)$.

As mentioned in the preceding subsection, the overdamped limit in the presence of an inhomogeneous friction amounts 469,471, 472, 488,489 to replacing $\eta$ by $\eta(x)$ in (2.17), (2.21). By means of the transformation

$$
\begin{aligned}
& \bar{x}(x):=\int_{0}^{x} d x^{\prime} \sqrt{\eta\left(x^{\prime}\right) / \bar{\eta}} \\
& \bar{\eta}:=\left[\int_{0}^{L} \frac{d x^{\prime}}{L} \sqrt{\eta\left(x^{\prime}\right)}\right]^{2}
\end{aligned}
$$

the Fokker-Planck equation for $P(\bar{x}, t)$ takes exactly the constant friction form 2.14) if one replaces $x$ by $\bar{x}$ and $\eta$ by $\bar{\eta}$. Including the "perturbations" $f(t), y(t)$, and $F$ (cf. (3.1)), the transformed equivalent Langevin equation takes the form

$$
\bar{\eta} \dot{\bar{x}}(t)=-\bar{V}^{\prime}(\bar{x}(t), f(t))+\sqrt{\bar{\eta} / \eta(\bar{x})}[y(t)+F]+\xi(t)
$$

where $\eta(\bar{x}):=\eta(x(\bar{x}))$ and

$$
\bar{V}(\bar{x}, f(t)):=V(x(\bar{x}), f(t))+\left(k_{B} T / 2\right) \ln (\eta(\bar{x}) / \bar{\eta}) .
$$

With (6.18), (6.19) one sees that $\bar{V}(\bar{x})$ and $\eta(\bar{x})$ exhibit again the same periodicity $L$ as $V(x)$ and $\eta(x)$.

In other words, we have mapped the original overdamped ratchet dynamics with inhomogeneous friction to our standard working model (3.1) with the only exception that 
the homogeneous external perturbation $[y(t)+F]$ acquires a spatially periodic multiplicative factor. Namely, an originally pure tilting ratchet now picks up some pulsating potential admixture, while a static force $F$ is now accompanied by a modification of the static part of the periodic potential profile. As a consequence, the basic qualitative features of such inhomogeneous friction ratchet models can be readily understood on the basis of our previously discussed results. For instance, a ratchet effect may now arise even if both $V(x, f(t))$ and $\eta(x)$ are symmetric according to (3.16) but each with a different $\Delta x$-value, i.e. they are out of phase, since this gives rise to a genuine effective ratchet potential with broken symmetry in (6.21). Regarding various interesting quantitative results for several specific models we refer to [190, 191, 471 473, 496 499].

An additional time-dependence of the friction $\eta(x)$ may arise under certain temporal variations of the system-plus-environment which are sufficiently slow in comparison with the characteristic relaxation time of the environment in order to always maintain an (approximate) accompanying equilibrium state of the bath. In such a case, the time dependence of $\eta(x, t)$ may be absorbed into the potential and the forces appearing on the right hand side of the properly rewritten original stochastic dynamics (3.1) similarly as in section 6.3. Afterwards, the remaining $x$-dependence can again be transformed away as in (6.20).

The special instance of a pulsating potential $V(x, f(t))$ in combination with a pulsating friction coefficient $\eta(x, f(t))$, both with the same periodicity in $x$, has been studied in the case of a dichotomous driving $f(t)$ in 474 . Since detailed balance symmetry gets lost in this way and in the absence of special symmetries, a ratchet effect is recovered [474] for such a pulsating friction ratchet. Especially, both $V(x, f(t))$ and $\eta(x, f(t))$ may be symmetric according to (3.16) but each with a different $\Delta x$-value, i.e. they are out of phase. As a further generalization, the transition probabilities between the two states of $f(t)$ may also periodically vary with $x$. Unless both of them are in phase with $\eta(x, f(t))$, a ratchet effect is then generically observed even in the absence of the potential| $V(x, f(t))$.

\subsubsection{Memory friction and correlated thermal noise}

Instead of forcing an unbiased $(F=0)$ system of the general form (3.1) by means of the perturbations $f(t)$ or $y(t)$ away from thermal equilibrium, one may as well consider a modification of the friction term $\eta \dot{x}(t)$ (while $f(t)=y(t)=0$ ). Much like in the previous subsection, the overdamped limit becomes then rather subtle and one better keeps a finite mass $m$ in the original description (2.1). The simplest such generalization [66, 79 81, 84, 89, 92 96] includes a so-called linear memory friction of the form

$$
m \ddot{x}(t)+V^{\prime}(x(t))=-\int_{-\infty}^{t} \hat{\eta}\left(t-t^{\prime}\right) \dot{x}\left(t^{\prime}\right) d t^{\prime}+\xi(t)
$$

see also sections 3.4.1 and 8.1 (the lower integration limit 0 in (8.4) is recovered from (6.22) by observing that $\dot{x}(t) \equiv 0$ for times smaller than the initial time $t=0$ ). The proper generalization (cf. (2.5), (3.2)) of the fluctuation-dissipation relation then

\footnotetext{
${ }^{5}$ A trivial example is: if $f(t)$ is in state 1 then for $x \in[0, L]$ the friction $\eta(x)$ is non zero only within $[0,3 L / 4]$ and the transition probability into state 2 only within $[L / 2,3 L / 4]$; if $f(t)$ is in state 2 then everything is shifted by $L / 2$.
} 
reads 66, 79 81, 84, 89, 92,96$]$

$$
\langle\xi(t) \xi(s)\rangle=\hat{\eta}(t-s) k_{B} T,
$$

see also (3.37), (3.38). Unless $\xi(t)$ is a stationary Gaussian process with zero mean and correlation (6.23), the environment responsible for the dissipation and fluctuations in (6.22) cannot be a thermal equilibrium bath [97] and therefore a ratchet effect is expected generically (and indeed observed), as exemplified in 119. Especially, the fact that some noise (Gaussian or not) is uncorrelated (white) does not necessarily imply that its origin is a thermal equilibrium environment nor does a correlated noise exclude thermal equilibrium.

\subsection{Ratchet models with an internal degree of freedom}

In this section we briefly review Brownian motors which posses - in addition to the mechanical coordinate $x$ - an "internal degree of freedom" analogous to the chemical state variable of molecular motors (cf. section 4.6 and chapter 7 ), but without the main intention of representing a faithful modeling of such intacellular transport processes. Another closely related model class are the two-dimensional tilting ratchet systems from section 5.5 .

So-called active Brownian particles [228, 500, 501] with an "energy depot" as additional internal variable have been considered in [502, 503] under the influence of a static ratchet potential. The internal energy depot models the capability to take up energy from the environment, store it, and (partially) convert it into directed motion. While the original, phenomenological model dynamics from [502, 503] does not fit into the generalized pulsating ratchet scheme from (7.3), it is possible to transform it into an equivalent form closely related to (7.3), namely a combined fluctuating potential and temperature ratchet with a back-coupling mechanism. Upon variation of the noise strength or of the energy supply, a remarkably rich behavior of the particle current $\langle\dot{x}\rangle$, both in magnitude and sign, is recovered [502,503].

A different type of "active Brownian particles", namely a reaction-diffusion system with one species of particles possessing a fluctuating potential ratchet type internal degree of freedom (chemical reaction cycle), has been demonstrated in [504] to induce a pattern forming process. Note also the connection of this setup with the collective ratchet models from chapter 9 .

A precursor of a two-headed motor enzyme model is the "elastic dumb-bell" from 41], consisting of two point-like Brownian particles which are linked by a (passive) elastic spring, and which move in either the same or two different (shifted) on-off ratchet potentials, see also [505] and [506] for the cases of fluctuating and traveling potential ratchet schemes, respectively. The corresponding "rocking-ratchet" situation, i.e. a static ratchet potential but a periodically varying external driving force, has been studied in [507] and extended in [508] and [184] to the cases when each of the two partcles moves in a two-dimensional ratchet potential and in a one-dimensional symmetric periodic potential, respectively. The analogous "fluctuating force ratchet" in the limit of a "rigid dumb-bell" has been considered in 371, 505, 509. Note that there exists a close connection to the models for single molecular motors in section 7.6, especially those in 451, 510 512.

A first experimental realization of such a two-head-like system with an active, spring-like element was reported as early as 1992 [513]: A curved strip of gel with 
periodically varying curvature (by externally applied electric fields) moves in a wormlike fashion with its two ends ("heads") along a ratchet-shaped substrate. A second experimental ratchet system with an "internal degree of freedom" was presented in [514]: A water droplet in oil is positioned on a ratchet shaped surface and its shape (internal degree of freedom) is periodically changed by means of externally applied electric fields. With the shape also the contact angles between the droplet and the surface change, with the result of a systematic directed motion. Since the droplet covers several periods of the ratchet, the rough picture is a somewhat similar wormlike motion as before, though the actual systems and their possible applications are of course completely different.

A Brownian particle in a periodic electric potential with an autonomously rotating "internal electric dipole" has been theoretically analyzed in [515]. Since the direction of this rotation breaks the spatial symmetry, the periodic potential may be chosen symmetric in this model. While there exists a close formal analogy with the traveling potential ratchet scheme from section 4.4.1, the physical picture is different 303.

\subsection{Drift ratchet}

In this section we discuss in some detail the so-called drift ratchet scheme 175 which resembles a rocking ratchet but at the same time goes substantially beyond our original tilting ratchet model from (5.1). We will outline the theoretical framework of a particle separation device based on this drift ratchet scheme, presently under construction 516 in the laboratories of the Max-Planck-Institut in Halle (Germany).

The system basically consists of a piece of silicon - a so-called silicon wafer - pierced by a huge number of identical pores with a ratchet-shaped (periodic but asymmetric) variation of the diameter along the pore-axis [516], see figure 6.2. The pores are filled with a liquid (e.g. water) which is periodically pumped back and forth in an unbiased fashion, i.e. such that no net motion of the liquid is produced on the average. Suspended into the liquid are particles of micrometer size and the objective is to separate them according to their size.

For a theoretical description of the particle motion we consider a single, infinitely long pore under the idealizing assumptions that the particles have spherical shape, that the suspension is sufficiently diluted such that particle interaction effects are negligible, and that the interaction with the pore walls can be captured by perfectly reflecting boundary conditions. For the typical parameter values of the real experiment, buoyancy effects due to the influence of gravitation as well as inertia effects of the particle are negligibly small, i.e., the particle dynamics in the viscous liquid is strongly overdamped. Assuming that the three-dimensional time-dependent velocity field $\vec{v}(\vec{x}, t)$ of the liquid is known, the particle $\vec{x}(t)$ is governed by the deterministic dynamics $\dot{\vec{x}}(t)=\vec{v}(\vec{x}(t), t)$. Here, $\vec{v}(\vec{x}, t)$ is, stictly speaking, not the velocity field of the fluid alone but rather the speed with which a spherical particle with center at $\vec{x}(t)$ and a small but finite radius is carried along by the surrounding liquid. This deterministic dynamics induced by the streaming liquid has to be complemented by the diffusion of the micrometer sized particle due to random thermal fluctuations $\vec{\xi}(t)$, which are caused by the impacts of the surrounding liquid molecules, and which we model in the usual way as Gaussian white noise. We thus end up with the following stochastic 


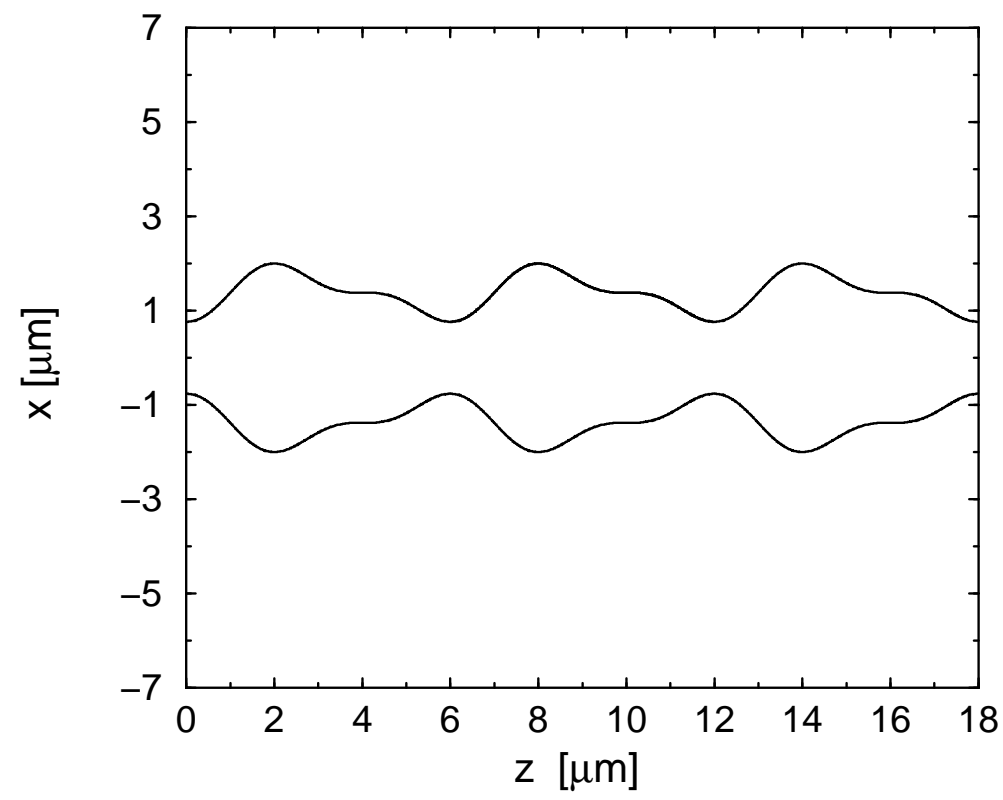

Figure 6.2: Schematic cross-section ( $x$-z-plane) through a single pore with an experimentally realistic, ratchet-shaped variation of the diameter along the pore axis $(z$ axis).

dynamics for the trajectory $\vec{x}(t)$ of a microsphere inside a single pore:

$$
\dot{\vec{x}}(t)=\vec{v}(\vec{x}(t), t)+\vec{\xi}(t) .
$$

The vector components $\xi_{i}(t), i=1,2,3$, of the noise $\vec{\xi}(t)$ are unbiased Gaussian processes with correlation

$$
\left\langle\xi_{i}(t) \xi_{j}(s)\right\rangle=\frac{2 k_{B} T}{\eta} \delta_{i j} \delta(t-s)
$$

The friction coefficient $\eta$ is in very good approximation given by Stokes law $6 \pi R \nu$, where $R$ is the particle radius and $\nu$ the viscosity of the liquid.

In view of the external, time-periodic pumping of the liquid through the pores, the above so-called drift-ratchet scheme has a certain similarity to a rocking ratchet system. On the other hand, it also remids one of the hydrodynamic ratcheting mechanism based on the the so-called Stokes drift [299 301, 303, 304 as discussed in the context of traveling potential ratchets in sections 4.4 and 4.5. However, in contrast to both, the rocking as well as the traveling potential ratchet paradigms, in the present case (6.24) no "ratchet-potential" is involved " Furthermore, the dynamics within a single pore is still a complicated three-dimensional problem that cannot be reduced in a straightforward manner to an effective one-dimensional model.

After one period of driving, the liquid in the pore returns to the same position from where it started out. Why should we not expect the same null-effect for the suspended particles? The basic reason is as usual the far from equilibrium situation, created

\footnotetext{
${ }^{6}$ Under the assumption of an incompressible fluid, i.e. $\vec{\nabla} \cdot \vec{v}=0$, the velocity field $\vec{v}$ appearing in (6.24) can be written as the curl of some vector potential, but never as the gradient of a scalar potential.
} 
in the present case by the periodic pumping, in combination with Curie's principle, which predicts the generic appearance of a preferential direction of the stochastic particle dynamics with broken spatial symmetry (6.24). The physical mechanism for the emergence of such a non-vanishing net particle current are the thermal diffusion between "liquid layers" of different speed and the collisions with the pore walls: Through the asymmetry of the pore-profile, an asymmetry between pumping forth and back arises for both the thermal inter-layer diffusion and the collisions with the pore-walls, resulting in a non-vanishing particle displacement on average after one driving period. The fact that the excursions of the particles during one driving period are typically much larger than the net displacement after one period (see figure 6.3) motivates the name "drift ratchet".

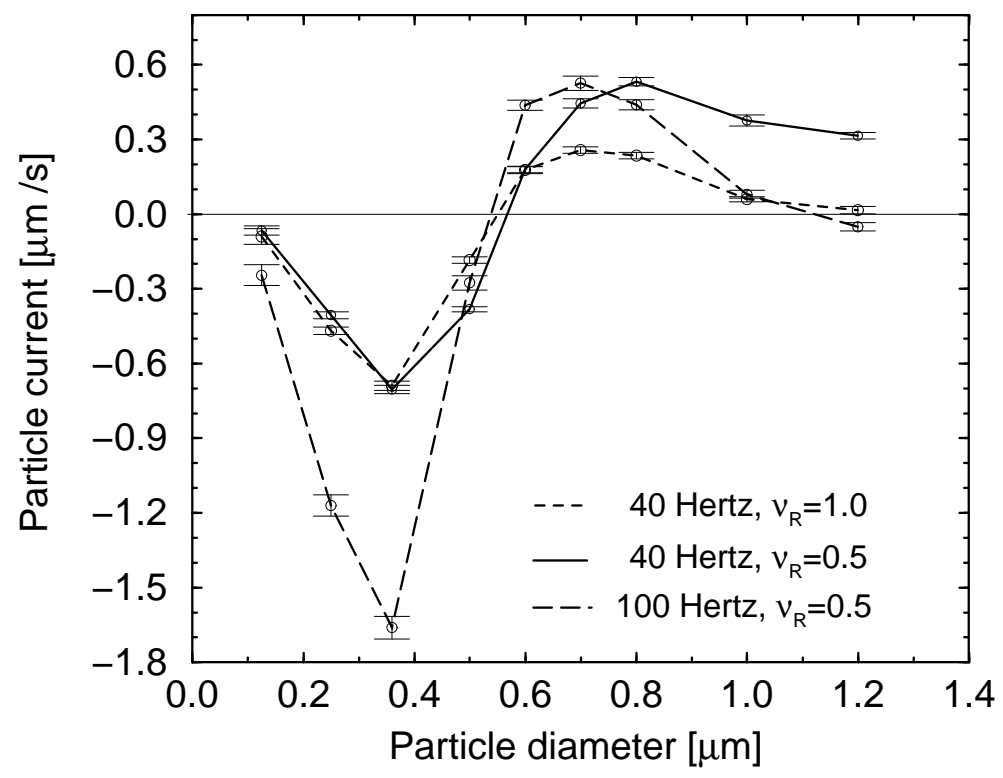

Figure 6.3: Numerical simulation of the stochastic dynamics (6.24), (6.25) for a pore shape as depicted in figure 6.2, at room temperature $(T=293 K)$. The friction coefficient $\eta$ in (6.25) is given by Stokes law $6 \pi R \nu$, where $R$ is the particle radius and $\nu=\nu_{\mathrm{R}} \nu_{\text {water }}$ the viscosity of the liquid in units of the viscosity $\nu_{\text {water }}$ of water. The velocity field in (6.24) has been obtained numerically with a sinusoidal pumping of the liquid at a frequency of $40 \mathrm{Hertz}$ and $100 \mathrm{Hertz}$. The pumping amplitude $A$ is chosen as $A=2 L$, where $L=6 \mu \mathrm{m}$ is the period of the ratchet-shaped pore in figure 6.2. Depicted is the time- and ensemble-averaged particle current $\langle\dot{z}\rangle$ along the pore axis ( $z$ axis) versus the particle diameter for various driving frequencies and viscosities.

The calculation of the velocity field $\vec{v}$ in (6.24) is a rather involved hydrodynamic problem in itself. For details of the necessary approximations (and their justification) in order to make the problem tractable at least by numerical methods we refer to [175]. Once such an approximation for $\vec{v}$ is available, the numerical simulation of the stochastic dynamics is straightforward. Typical results for realistic parameter values are depicted in figure 6.3, demonstrating that the direction of the particle current depends very sensitively on the size of the particles.

While, according to section 3.6, such current inversions are a rather common phenomenon, the distinguishing feature of our present device is its highly parallel architec- 


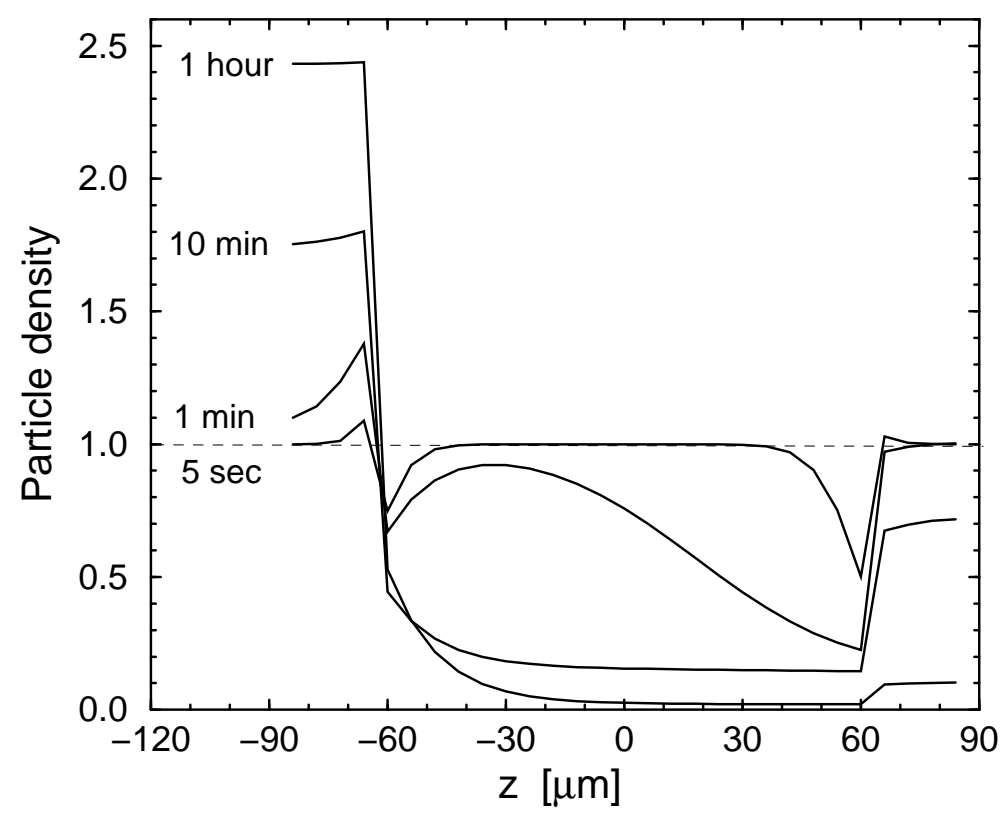

Figure 6.4: Time evolution of the particle density (within the liquid-plus-particle suspension) along the $z$-axis starting with a homogeneous initial distribution (normalized to unity). The pore length (along the $z$ axis) is $126 \mu m$ and the extension $\Delta z$ of each of the two adjacent basins along the $z$ axis is $24 \mu \mathrm{m}$. Other details are like in figure 6.3 with particle radius $R=0.36 \mu \mathrm{m}$, pumping frequency $100 \mathrm{Hertz}$, pumping amplitude $A=L$, and relative viscosity $\nu_{R}=0.5$.

tur£: a typical silicon waver contains about one million pores per square centimeter. On the other hand, the pores in a real silicon wafer are not of infinite length - as so far assumed - but rather the wafer is connected at both ends to basins of the liquidplus-particle suspension and the actual pumping device. For practical applications, not the steady state current in an infinite pore is of main importance, but rather the time needed to achieve reasonably large concentration differences between the two basins (see also the discussion below equation (3.7)). We now focus on the case of two identical basins, each of an extension $\Delta z$ along the $z$ axis and of the same cross section as the wafer (perpendicular to the $z$ axis). The typical time evolution of the particle density for such a setup is depicted in figures 6.4 and 6.5. These calculations predict a remarkable theoretical separating power of the device. Its experimental realization - presently under construction [516] - thus appears to be a promising new particle separation device, possibly superior to existing methods for particles-sizes on the micrometer scale.

\subsection{Spatially discrete models and Parrondo's game}

The spatially discretized counterpart of our working model (3.1) arises when the state variable $x(t)$ is restricted to a set of discrete values $x_{i}$. In the simplest case, the

\footnotetext{
${ }^{7}$ We remark that also the experimental systems from 38,273, 277] (discussed in section 4.2.1 and at the end of section 4.4.1) include a parallelization in two dimensions, while in the present case three dimensions are exploited.
} 


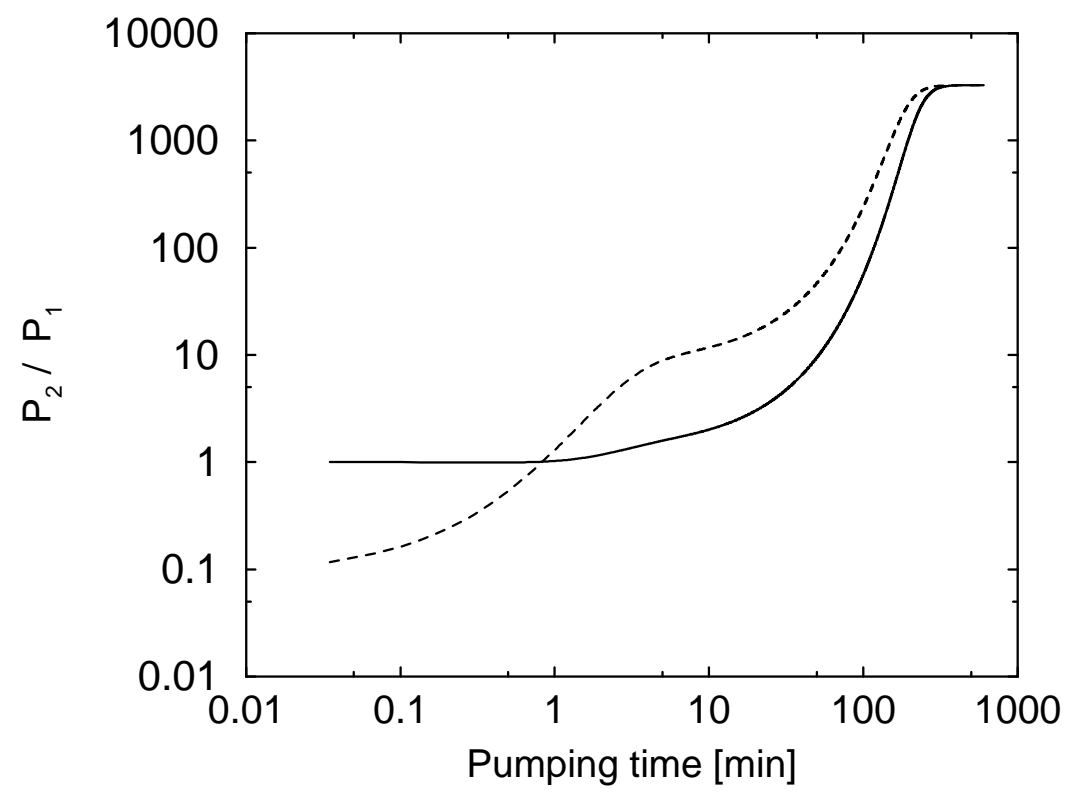

Figure 6.5: Ratio $P_{2} / P_{1}$ of particle densities for two types of particles versus time $t$. The setup is the same as in figure 6.4 but with a pumping amplitude $A=2 L$ and with radii of the two types of particles $R_{1}=0.36 \mu \mathrm{m}$ and $R_{2}=0.7 \mu \mathrm{m}$ (corresponding to opposite current directions in figure 6.3). The ratio of the densities $P_{2} / P_{1}$ refers to the border of the right basin at $z=87 \mu m(=126 / 2 \mu m+24 \mu m)$. Solid line: Overall homogeneous initial densities. Dashed line: Initially homogeneous densities in the pore region and vanishing densities in the two basin regions.

time evolution is given by a so-called Markov-chain dynamics, i.e. transitions are only possible between neighboring states $x_{i}$ and $x_{i \pm 1}$, and they are governed by transition rates $k_{i \rightarrow i \pm 1}(t)$, which in general may still depend on time. As a consequence, the probability distribution $P_{i}:=\left\langle\delta\left(x_{i}-x(t)\right)\right\rangle$ evolves in time according to a master equation of the form

$$
\dot{P}_{i}(t)=-\left[k_{i \rightarrow i+1}(t)+k_{i \rightarrow i-1}(t)\right] P_{i}(t)+k_{i+1 \rightarrow i}(t) P_{i+1}(t)+k_{i-1 \rightarrow i}(t) P_{i-1}(t) .
$$

The spatial periodicity of the system implies that there is an integer $l$ with the properties that

$$
\begin{aligned}
x_{i+l} & =x_{i}+L \\
k_{i+l \rightarrow j+l}(t) & =k_{i \rightarrow j}(t)
\end{aligned}
$$

for all $i$ and $j$.

A periodic Markov-chain model (6.26)-( $(6.28)$ may arise in several different contexts. The most prominent is the activated barrier crossing limit as discussed in section 3.8, i.e., the spatially continuous dynamics (3.1) is characterized by rare transition events between metastable states $x_{i}$. In the simplest (and most common) case $l=1$, i.e. there is only one metastable state $x_{i}$ per spatial period $L$ and the rates are - possibly after temporal coarse graining (see section 3.8) - independent of time. While the actual calculation of those rates $k_{ \pm}:=k_{i \rightarrow i \pm 1}$ is in general highly non-trivial, once they are 
given, the determination of the current and the diffusion coefficient is straightforward, see (3.55), (3.56) and the footnote on page 56 .

The problem of calculating the rates $k_{i \rightarrow i \pm 1}(t)$ simplifies a lot if the characteristic time scale of the driving $f(t)$ and/or $y(t)$ in (3.1) is much larger than the intrawell relaxation time within any metastable state (but not necessarily larger than the characteristic interwell transition times $1 / k_{i \rightarrow i \pm 1}(t)$ themselves). Under these circumstances, an adiabatic approximation like in section 2.10 can be adopted, with the result that at any given time $t$, the rates $k_{i \rightarrow i \pm 1}(t)$ are given by a Kramers-Smoluchowski type expression analogous to (2.45). Comparing (3.1) with (2.34), we see that in those rate expressions not only the instantaneous effective potential $V_{\text {eff }}(x, t)=V(x, f(t))-x y(t)-x F$ (cf. (2.35)) depends on $f(t)$ and/or $y(t)$, but also the locations $x_{\min }=x_{i}$ of the metastable states (local minima) and of the activated states (local maxima) $x_{\max }$. Besides the slow variations of $f(t)$ and/or $y(t)$, the implicit assumptions of this approximation are that the number of metastable states within one spatial period $L$ is the same for all times $t$, that their position changes in the course of time continuously or with not too big jumps, and that the potential barriers between any two of them is much larger than the thermal energy $k_{B} T$. Within these restrictions, any spatially continuous class of ratchets from section 3.3 immediately entails a spatially discretized counterpart. Especially, we note that the characteristic features of the diffusion ratchet scheme will be a time-dependent temperature $T(t)$ in the Kramers-Smoluchowski rates (2.45), while for a Seebeck ratchet (section 6.1), the effective barriers $\Delta V_{\text {eff }}$ and pre-exponential factors in (2.45) have to be calculated along the lines of section 4.3.2.

Along this general ideology, the spatially discretized on-off ratchet scheme (see section 4.2. has been worked out in [172, 517], while a modified on-off description of a Feynman ratchet (see section 6.2]) is due to [484]. As another variation, an asynchronously pulsating on-off model (cf. section 3.4.2) has been put forward in [197]. In such a model, (6.28) is no longer satisfied and instead within each spatial period $L$ the potential switches independently between its on- and an off-state. If these switching events within neighboring periods are correlated or anticorrelated, the current is enhanced as compared to the completely uncorrelated case [197]. Related, spatially continuous, asynchronously pulsating ratchet models have been studied in 198 202].

Spatially discretized pulsating ratchet models have been addressed in [129, 518], temperature ratchets in [128, 129], traveling potential ratchets in [164], and rocking ratchets in 129, 172, 518], see also [233, 234, 236, 237, 519] for the case of extremely slow rocking.

For biological intracellular transport processes (cf. sect 4.6 and chapter 7), spatially discretized descriptions arise naturally and have been analyzed in detail e.g. in 8, 9, 16, 21, 22, 186, 187, 520 526.

In all those works, the above mentioned approximation of the rates $k_{i \rightarrow i \pm 1}(t)$ by instantaneous Kramers-Smoluchowski type expressions (2.45) have been exploited. The advantage of such an approach is that closed analytical solutions can often be obtained, especially if the driving $f(t), y(t)$, and/or $T(t)$ jumps (either periodically or randomly) between only a few different values. Since the main qualitative findings are very similar as for the spatially continuous case (see chapters 45 ) we do not discuss these features in any further detail at this place. We only remark that if the spatially continuous model leads to a vanishing current in the adiabatically slow driving case (e.g. for fluctuating potential and temperature ratchets), then at least two metastable states $x_{i}$ per period $L$ (i.e. $l \geq 2$ ) are required for a ratchet effect in the spatially 
discrete counterpart [517]. In any other case, one metastable state $x_{i}$ per period $L$ (i.e. $l=1$ ) is sufficient. Such spatially discretized, adiabatically driven models with a minimal number $l$ of states per period are sometimes called minimal ratchets in view of their mathematical and conceptual simplicity.

We emphasize again that while discrete models are usually easier to analyze than their spatially continuous counterparts, the actual hard problem has now been shifted to justifying such a discretized modeling and to determine the rates ("phenomenological model parameters") either from a more detailed (usually continuous) description (cf. section 3.8) or from experimental observations.

A second context in which a spatially discretized dynamics (6.26) arises is the numerical method for solving the originally continuous problem (3.1) which has been introduced in 361 and applied to various specific models in 162, 250, 369, 527, 528. Chosing the rates $k_{i \rightarrow i \pm 1}(t)$ according to the recipe from [361], this numerical scheme approximates the solution of the continuous system better and better as the number of states $l$ per period increases. Conversely, the often analytically solvable models with only very few states $x_{i}$ per period $L$ may be still considered as a first rough approximation of the spatially continuous problem.

Another cute application of the discretized on-off ratchet scheme has been invented by Parrondo [529 535]. Namely, the spatially discretized random dynamics for both the on- and the off-configurations of the potential are re-interpreted as games, and by construction each of these two games in itself is fair (unbiased). The astonishing phenomenon of the ratchet effect then translates into the surprising observation that by randomly switching between two fair games one ends up with a game which is no longer fair. This so-called Parrondo paradox is thus in some sense the game theoretic transfiguration of Brillouin's paradox from section 2.9. Generalizations are obvious: For instance, by switching between two games, each (weakly) biased into the same direction, the resulting game may be biased just in the opposite direction. Another option is to take as starting point for the translation into a game a ratchet model different from the on-off scheme [536, 537], and so on.

\subsection{Influence of disorder}

In this section we briefly review some basic effects which arise if the periodicity of the potential (3.3) is modified by a certain amount of quenched spatial disorder. Our starting point is an "unperturbed", strictly periodic system in the activated barrier crossing limit as discussed in section 3.8, i.e. transitions between neighboring spatial "cells" of length $L$ can be described by "hopping"-rates $k_{+}$and $k_{-}$to the right and left, respectively. Without loss of generality we furthermore assume that the unperturbed current $\langle\dot{x}\rangle$ in $(3.55)$ is positive, i.e.

$$
k_{+}>k_{-}
$$

In the simplest case we may now introduce a quenched randomness as follows: For each pair of neighboring cells we interchange with a certain probability $p$ the original transition rates $k_{+}$and $k_{-}$to the right and left. For instance, in a piecewise linear "sawtooth-potential" as depicted in figure 4.1, such an interchange of the transition rates can be realized by randomly inverting the orientation of each single saw-tooth with probability $p$ independently of each other. Without loss of generality we can 
restrict ourselves to probabilities

$$
0 \leq p \leq 1 / 2
$$

The following basic effects have been unraveled by Derrida and Pomeau [233, 234]: Upon increasing $p$ the particle current $\langle\dot{x}\rangle$ monotonically decreases from its initial value (3.55) and vanishes for $p \geq p_{1}$, where

$$
p_{1}:=\frac{k_{-}}{k_{+}+k_{-}} .
$$

More precisely, for $p \geq p_{1}$ the mean displacement $\langle x(t)\rangle$ grows asymptotically slower than linearly with $t$. The effective diffusion coefficient (3.6) increases monotonically from its unperturbed value (3.56) and diverges at $p=p_{2}$, where

$$
p_{2}:=\frac{k_{-}^{2}}{k_{+}^{2}+k_{-}^{2}} .
$$

For $p_{2} \leq p \leq p_{3}$, where

$$
p_{3}:=\frac{\sqrt{k_{-}}}{\sqrt{k_{+}}+\sqrt{k_{-}}},
$$

a superdiffusive behavior arises $\left(D_{\text {eff }}=\infty\right)$, i.e. the dispersion $\left\langle[x(t)-\langle x(t)\rangle]^{2}\right\rangle$ grows asymptotically faster than linearly with $t$, switching over [538] to a subdiffusive behavior (slower than linear growth of the dispersion, i.e. $D_{\text {eff }}=0$ ) for $p_{3}<p \leq 1 / 2$. (Note that $0<p_{2}<p_{1}<p_{3}<1 / 2$ ). At least in the regimes where they are finite, the quantities $\langle\dot{x}\rangle$ and $D_{\text {eff }}$ are self-averaging, i.e. the same (finite) value is observed with probability 1 for any given realization of the quenched disorder. A simple intuitive explanation of these results does not seem possible, which may not be so surprising in view of the above mentioned self-averaging issue and other subtle problems of commuting limits in this context, see [235, 538, 539] and references therein.

More general, but still uncorrelated randomizations of the transition rates between pairs of neighboring "cells" of length $L$ are given already by Derrida in [234]. A variety of cases with correlated randomization has been discussed in [540] (see also the review [538]) together with several interesting physical applications.

A bold but apparently quite satisfactory approximative extension beyond the activated barrier crossing limit has been proposed in [171]: The basic idea is to evaluate, either analytically or numerically, for the unperturbed (strictly periodic) ratchet dynamics both the current $\langle\dot{x}\rangle$ and the diffusion coefficient $D_{\text {eff }}$. Introducing these results for $\langle\dot{x}\rangle$ and $D_{\text {eff into } 3.55)}$ and (3.56) yields formal expressions for the rates $k_{ \pm}$even though these rates no longer adequately describe the actual transitions between neighboring "cells". Assuming that a randomization of the ratchet potential can still be captured by a corresponding randomization of the formal forward and backward rates $k_{ \pm}$, one thus can continue to use Derrida and Pomeau's formulas 2233, 234 for an approximative description of such a randomized ratchet dynamics. For the example of an on-off ratchet scheme, a fair agreement of this approximative approach with accurate numerical simulations has been reported in 171.

Another, more systematic first step beyond the activated barrier crossing limit is due to [541], considering a fluctuating force ratchet with a very general disordered 
potential $V(x)$ that is (additively) driven by asymptotically weak symmetric Poissonian shot noise (cf. section 5.1).

A deterministic $(T=0)$ rocking ratchet model with quenched spatial disorder has been addressed in [542]. Similarly as before, the current decreases and the (deterministic) diffusion accelerates with increasing disorder, but apparently these quantities no longer exhibit the experimentally important self-averaging property. Results more in accordance with the above described standard scenario of Derrida and Pomeau are recovered upon including inertia effects [101]. A similar overdamped case but with finite $T$ and adiabatically slow rocking has been addressed in [543].

\subsection{Efficiency}

The issue of efficiency of Brownian motors has recently developed into an entire subfield of its own right. Here, we resrict ourselves to a very short overview.

The most widely accepted definition of the efficiency for a ratchet dynamics of the general form (3.1) is given by the ratio $R$ of the average mechanical work per time unit $\langle\dot{x}\rangle F$ produced by the the "energy transducer" $x(t)$ and the average net power input $\left\langle P_{\text {in }}\right\rangle$ stemming from the external driving $f(t)$ and/or $y(t)$, i.e.

$$
R:=\frac{\langle\dot{x}\rangle F}{\left\langle P_{\text {in }}\right\rangle} .
$$

Both averages in this equation are meant with respect to all random processes and time-periodicities involved in (3.1) and transients are assumed to have died out. For ergodicity reasons, both averages can then also be rewritten as long time averages for a single realizations of the stochastic dynamics (3.1), cf. (3.5). In order to quantitatively calculate the efficiency (6.34) for the different classes of ratchet models (3.1), a very general and elegant framework has been developed by Sekimoto [324, 480, 544 546], unifying and putting on firm grounds the various previously proposed, model-specific expressions for $\left\langle P_{\text {in }}\right\rangle$ in (6.34).

As pointed out in section 3.4.2, the origin of a random external driving $f(t)$ and/or $y(t)$ may be conceived as a thermal heat bath, very weakly coupled to the system variable $x(t)$ in order that back-coupling (friction-type) effects are negligible 8 , but at a temperature much higher than the temperature $T$ of the thermal noise $\xi(t)$. From the viewpoint of a Carnot machine, the temperature $T$ is thus to be associated with the cooler heat bath and the maximally achievable Carnot efficiency is practically $100 \%$. If the external driving is not random but periodic, it is quite suggestive that the same conclusion with respect to the achievable efficiency still carries over.

While it is not yet clear whether efficiency issues are of major relevance in practical applications of the ratchet effect or for intracellular transport processes, their principle interest has stimulated a considerable amount of theoretical studies. Apparently the first such discussion goes once again back to Feynman's lectures [2], though the conclusion that under certain conditions the maximal Carnot efficiency is reached, cannot be upheld f against more detailed studies of microscopic Feynman ratchet mod-

\footnotetext{
${ }^{8}$ We remark that Sekimoto's framework 324, 480, 544 546 for evaluating the efficiency in $(6.34)$ remains valid even when such back-coupling effects are included, as it is the case e.g. for the Feynman ratchet model in section 6.2 or the molecular motors studied in chapter 7 .

${ }^{9}$ The same misguided method of calculating efficiencies has been adopted in 7 , see also 481, 482].
} 
els [110, 111, 480, 481,547, see also section 6.2. For a representative example, a maximal efficiency of the order of $10^{-4}$ has been obtained in 4480].

Efficiency aspects of photovoltaic and photorefractive effects in non-centrosymmetric materials (see section 5.2) are surveyed in [28]. Typical values of the efficiency in real materials are found to be $10^{-3}-10^{-2}$, while theoretical situations with up to $100 \%$ efficiency are conceivable [28]. The existence of so-called reversible ratchet models which achieve in certain limits the maximal possible efficiency of $100 \%$ has also been demonstrated for rocking ratchets 10 in [549, 550], for generalized pulsating ratchets (i.e. neither of the purely fluctuating nor traveling potential type) in [198, 199, 201, and for an even more general class of models in [551]. A first condition for reaching the maximal Carnot efficiency of $100 \%$ is that the system is at every given time instant in a quasi equilibrium state [552]. Especially, all temporal variations due to the external driving $f(t)$ and/or $y(t)$ must be adiabatically slow. A second condition is 198, 199, 201 that the current $\langle\dot{x}\rangle$ approaches zero not faster than proportional to the inverse characteristic time scales of $f(t)$ and $y(t)$ in the adiabatic limit. E.g. for fluctuating potential ratchets and the closely related temperature ratchets (cf. section 6.3), the maximal Carnot efficiency can not be reached since the latter condition is not met (see section 4.1).

The efficiency of a Seebeck ratchet model (see section 6.1) with a temperature $T(x)$ which varies periodically in space between two different values $T_{1}$ and $T_{2}>T_{1}$, has been addressed in [476, 546, 553, 554. According to [476, 553], for a suitable choice of the model parameters, an efficiency arbitrarily close to the maximal Carnot efficiency $\left(T_{2}-T_{1}\right) / T_{2}$ can be reached. The above mentioned requirement that the system is at any time in a quasi equilibrium state may then be granted by the overdamped limit $m \rightarrow 0$, entailing a vanishingly small relaxation time of the particle $x(t)$ towards local thermal equilibrium. On the other hand, in [546, 554] it is argued that Carnot efficiency is unattainable in such systems. A related model of a non-isothermal electrical circuit with a diode (thermogenerator, see section 6.1) has been analyzed from the viewpoint of efficiency in [157]. While this one-diode setup, much like its mechanical Feynman ratchet counterpart, always leads to an efficiency smaller than the Carnot value, an extended model with two diodes may approach this theoretical upper limit for the efficiency arbitrarily close [555], demonstrating that even a system which is simultaneously in contact with more than one heat bath may still operate reversibly, see also 550.

Universal, i.e. largely model-independent features of efficiencies for ratchet models close to thermal equilibrium (Onsager regime of linear response) have been worked out in 114,484, 544, 551. Remarkably, by moving out of the linear response regime into the far from equilibrium realm the efficiency may not necessarily decrease [551]. Similarly, for some ratchet models, the efficiency may even increase upon increasing the temperature $T$ of the thermal heat bath both, for systems near 551 and far from equilibrium [129, 528, 556], in contrast to what one would expect from a Carnot efficiency point of view.

As already mentioned, fluctuating potential ratchets and temperature ratchets cannot reach the maximal Carnot efficiency. Specifically, the on-off scenario leads under typical conditions to efficiencies of a few percent [198, 517, 527, 528, 551]. However, in the case that many on-off ratchet are coupled together (see section 7.4 .4 ) the efficiency

\footnotetext{
${ }^{10}$ However, under typical conditions only a few percent are actually reached 499,548].
} 
may again reach values of $50 \%$ and beyond [14,557. Efficiencies of at most a few percent have also been reported for fluctuating potential ratchets (see section 4.3) [129], temperature ratchets (see section 6.3) 128 130, and coupled rocking ratchets [558 (see equation (9.34)).

Based on experimental measurements of intracellular transport processes, the possibility that the molecular motor kinesin (cf. chapter 7) may reach an efficiency as high as $50 \%$ or even $80-95 \%$ is discussed in [525] and [521,522, respectively, see also [303,559].

Other definitions of efficiencies than in (6.34) have been introduced and discussed in 12, 284, 303, 547, 551, 560 564. Related quantities like entropy production, Kolmogorov information entropy, and algorithmic complexity have been explored in [190, 484, 527, 528]. Evidently, with respect to the definitions of such alternative efficiency-type quantities it does not make sense to ask whether they are "right" or "wrong" (apart from the trivial requirement that they are "well-defined" in the mathematical sense). Rather, the crucial question regards their usefulness [551]. For instance, it may be possible to agree on one such quantity as being a particularly appropriate quality measure in a certain context [12]. In many cases this will indeed be the standard "efficiency for generating force" (6.34). However, in other cases, it may be important to accomplish a certain task not only by means of a minimal amount of input energy - as in (6.34) - but in addition within a prescribed, finite amount of time. This constrained optimization task is the basis of the alternative "efficiency of transprotation" concept from [303, 561, 562], which has been to some extent anticipated in [560], and which is also closely related to the issue of finite-time thermodynamics [565 567]. For further details, we refer to the above cited original works, see also at the end of section 8.4 . 


\section{Chapter 7}

\section{Molecular motors}

In this chapter we exemplify in detail the typical stochastic modeling procedure by elaborating the general scheme from section (4.6) for a particularly important special case of intracellular transport, namely so-called motor enzymes or molecular motors which are able to travel along polymer filaments inside a cell. Specifically, we shall focus on molecular motors from a subfamily of the the so-called kinesin superfamily, which are capable of operating individually. For the two other main superfamilies of motor enzymes (myosin and dynein) many of the basic qualitative modeling ingredients remain the same as for kinesin, while the details are different 568, 569; we will briefly address the case of molecular motors which only can operate collectively, e.g. the socalled myosin II subfamily, later in this chapter. More involved variants of intracellular transport like rotary mechanochemical energy transducers are treated e.g. in [570 574. Also not covered by the present chaper are "Brownian ratchets" - a notion which has been coined in a rather differen context, namely as a possible operating principle for the translocation of proteins accross membranes [121 125]. A collection of computer animations which visualize several of these intracellular transport processes is available on the internet under [575].

\subsection{Biological setup}

The most primitive living cells are the so-called prokariotes, i.e. cells without a nucleus (mostly bacteria) [346]. Their interior is basically one large soup without any internal partition. Since prokaryotic cells are at the same time very small, the intracellular transport of various substances can be accomplished passively, namely through thermal diffusion. In contrast, eucaryotic cells (the constituents of any multicellular organism) are not only higher organized but also considerably larger so that passive diffusive transport becomes too inefficient [576]. Their distinguishing features are the existence of a cell nucleus (responsible for the storage and transcription of the genetic material), many other internal compartments, called organelles, and a network of polymer filaments - the "cytoskeleton" - which organizes and interconnects them. These filaments radiate from a structure near the nucleus called the centrosome to the periphery of the cell and so support the shape of the cell. Besides several other intracellular functions, which go beyond our present scope, they act as a circulatory system, connecting and feeding distinct regions of the cell. They are paths along which nutrients, wastes, proteins, etc. are transported in packages, called vesicles, by specific 
motor proteins (mechanoenzymes).

One major type of such polymer filaments are fibers of proteins called microtubuli, with the constituent protein "tubulin" - a dimer of two very similar globular proteins ( $\alpha$-tubulin and $\beta$-tubulin) about $4 \mathrm{~nm}$ in diameter and $8 \mathrm{~nm}$ long [346]. The microtubulus is composed of typically 13 protofilaments (rows of tubulin-dimers) that run parallel to the axis of the fiber. The emerging shape of the microtubulus resembles that of a hollow, moderately flexible tube with an outer diameter of about $25 \mathrm{~nm}$, and inner diameter of about $17 \mathrm{~nm}$, and an overall length of up to a few $\mu \mathrm{m}$. Due to the asymmetry of the tubulin-dimers, the tube has a polarity, one end exposes only $\alpha$-tubulin, and the other only $\beta$-tubulin. On top of that, the tube exhibits a definite chirality or helicity since the dimer-rows of neighboring parallel protofilaments are shifted against each other.

One specific motor enzyme which can travel on a microtubulus and pull along various objects like chromosomes, viruses, or vesicles with chemicals in it, is the protein "kinesin" [346,568. The necessary energy to move against the viscous drag is supplied by the so-called ATPase, i.e. the exothermic chemical hydrolysis of ATP (adenosine triphosphate) into ADP (adenosine diphosphate) and $\mathrm{P}_{i}$ (inorganic phosphate). The shape of a single kinesin molecule is rather elongated, about $110 \mathrm{~nm}$ in length and about $10 \mathrm{~nm}$ in the other two spatial directions. One of its ends consists of a bifurcated "tail", capable of grasping the cargo to be carried, then follows a very long rod-shaped middle segment, the $\alpha$-helical coiled-coil stalk, while the other end bifurcates into two identical globular "heads" or "motor domains" 143,577. In spite of the nomenclature, the functioning of the heads is actually quite similar to that of human legs, proceeding along the microtubulus in a "step-by-step" or "hand-over-hand" fashion [578]. We emphasize that the comparison with the walking of a human is common but should not be overstretched: There is evidence that the bound head in fact produces a rotation that "swings" the second head towards its next binding site [143, 568. The reason is that the kinesin as a hole seems to possess an (approximate) axis of rotational symmetry by $180^{\circ}$, implying that we should think of the two heads not as "right and left feet" but rather as "two left feet" [143]. Each single foot, on the other hand, does not share this (spatial inversion-) symmetry, it has well distinguishable "heel" and "fingers".

Each head comprises in particular a microtubulus-binding site as well as an ATPbinding site, called the ATP-binding pocket. Accordingly, each head can bind and hydrolyze ATP on its own. The underlying chemical reaction cycle consists of the following four basic steps (and corresponding states) with the result of about $20 k_{B} T$ energy gain per cycle [9]: State 1: The motor domain is interacting with the environment and attached to the microtubulus, but without anything else bound to it. Transition into state 2: The head binds one ATP molecule out of the environment in its ATP binding pocket. Transition into state 3: The ATP is broken up into ADP and $\mathrm{P}_{i}$ - the so-called power-stroke - with the above mentioned energy gain of about $20 k_{B} T$. Transition into state 4: The $\mathrm{P}_{i}$ is released from the ATP binding pocket and simultaneously the affinity to the microtubulus decreases dramatically, so that the head typically detaches. Transition into state 1: The ADP is released, the affinity to the binding sites ( $\beta$-tubulin) of the microtubulus becomes again large, with the result that the head will, after some random diffusion, attach to one of them, and we are

\footnotetext{
${ }^{1}$ Additional intermediate steps can be identified [579] but are usually neglected due to their short lifetimes.
} 
back in state 1 .

The "energy factories" of the cell are constantly supplying fresh ATP and removing the used ADP and $\mathrm{P}_{i}$, thereby keeping the concentration of ATP inside the cell about 6 decades above its thermal equilibrium (detailed balance) value, so that the probability of an inverse (endothermic) chemical cycle, transforming ADP and $\mathrm{P}_{i}$ back into ATP is completely negligible.

It is noteworthy that the heads do not hydrolyze ATP at any appreciable rate unless they interact with the microtubulus, indicating that at least part of the chemical cycle is intimately coupled to the binding to a microtubulus [578]. The hydrolyzing step takes place while the head is attached to the microtubulus; the subsequent release of $\mathrm{P}_{i}$ enables the head to release its hold so that it can take another step on its journey along the microtubulus. The key to the energy transduction is thus the large change in affinity between the heads of the motor protein and the protein filament on which it walks. A particularly strong affinity develops between the microtubulus-binding site of a head and the $\beta$-tubulin monomers. As a consequence, each tubulin dimer can bind at most one head and thus a single head has to cover the length of two dimers (about $16 \mathrm{~nm}$ ) during each step of the motor enzyme along the microtubulus. To complete the picture, it should be mentioned that the motor enzyme proceeds along the microtubulus in a straight way, it does not "spiral" around the hollow tube during its journey [143, 508,578, 580]. Rather it follows with high fidelity a path parallel to the protofilaments so that the helicity of the microtubulus most likely plays no essential role; the main origin of the spatial asymmetry as far as the kinesin walk is concerned is that of the constituent dimers of the microtubulus together with that of the binding sites of the single heads. Remarkably, each given species of the kinesin superfamily can travel only in one preferential direction along the microtubulus, but different species may move in opposite directions though they may be of striking structural similarity [143, 577, 581 584.

Kinesin is a so-called processive motor enzyme, that is, it can operate individually. A single kinesin molecule can cover a distance of the order of $1 \mu \mathrm{m}$ before it may loose contact with the microtubulus and diffuses away [568, 577, 585, 586], and this possibly even against an opposing force of up to 5 piconewtons [142, 587]. The reason seems to be on the one hand that the time-interval during which a single head is detached from the microtubulus while "stepping forward" is relatively short (one speaks of a high "duty ratio") and on the other, that the two heads coordinate their actions so that at least one head is always attached [579]. A striking manifestation of this coordination is the fact [143, 568] that apparently it is the energy gain out of the power stroke of the "front" head which triggers the "rear" head to make a step forward.

For a more detailed exposition of the biophysical basics and experimental findings we refer to the excellent recent monograph by Howard [525].

\subsection{Basic modeling-steps}

\subsubsection{Biochemical framework}

Our first step in modeling a motor enzyme consists in recalling the description of a general biochemical reaction [185,588 594]: In principle, the starting point should be a quantum chemical $a b$ initio treatment of all the electrons and atomic nuclei of the molecules involved in the reaction. Due to the clear cut separation of electron and 
nuclei masses, the electron dynamics can be adiabatically eliminated for each fixed geometrical configuration of the nuclei (Born-Oppenheimer approximation [589, 591]) with the result of an effective potential energy landscape for the nuclei's motion alone. In principle, there are many quantum mechanical energy eigenstates of the electrons for any fixed configuration of the nuclei, giving rise to a multitude of possible "potential energy surfaces" in the configuration space of the nuclei [589,591,593]. We assume that only one of them (the ground state energy of the accompanying electrons) is relevant in our case and especially is always well separated from all the other potential energy surfaces. In other words, the effective potential landscape governing the dynamics of the nuclei is single valued and no excitations of the electronic states are involved in the reaction cycle. Since the nuclei are already fairly massive objects, quantum mechanical effects will often play only a minor role for their dynamics, and we can focus on an approximate classical treatment. Indeed, while for very simple chemical reactions, a semiclassical or fully quantum chemical treatment may be necessary and still feasible, classical molecular dynamics is the only practically realistic approach in the case of a complex biomolecular system with hundreds or thousands of atoms, as we consider it here. In other words, all the relevant quantum mechanics of the system is assumed to be already encapsulated in the effective potential in which the nuclei move?

So far our description still comprises both the molecular motor and its environment, typically some aqueous solution containing in particular ATP, ADP, and $\mathrm{P}_{i}$ molecules in certain concentrations. The role of the environment is twofold: On the one hand, it acts as a heat bath, giving rise to randomly fluctuating forces and to the associated damping (energy dissipation) mechanism in the molecular motor's dynamics. On the other hand, it represents a source and sink of the reactants (ATP molecules) and products (ADP and $\mathrm{P}_{i}$ ) of the chemical reaction cycle.

The influence of that part of the environment which acts as thermal heat bath can be taken into account along the same line of reasoning as in sections 2.1 .2 and 3.4.1. The result is a classical stochastic dynamics for the motor enzyme with a certain type of random noise and dissipation term, possibly supplemented by a renormalization of the effective potential landscape and the nuclear masses [92,93. Under the assumption that the typical potential barriers are large compared to the thermal energy $k_{B} T$, the configurations of the motor enzyme (defined by the coordinates of the nuclei) will be restricted for most of the time to the local minima (metasable states) of the potential landscape and small fluctuations there aboutf, while transitions between different local minima are rare events.

In the case of simple chemical reactions, these transitions furthermore occur practically always along the same "most probable escape path", called also "chemical pathway", "reaction path", or "intrinsic reaction coordinate" in this context [588, 592, 593]. One thus can describe all the essential configurations of the reaction in terms of this single intrinsic reaction coordinate and small (thermal) fluctuations there about. The latter can again be taken into account by means of dissipation and fluctuation terms in complete analogy to the above mentioned modeling of the thermal heat

\footnotetext{
${ }^{2}$ Several of the above assumptions are in fact not necessary for the validity of our final reduced description (see below), i.e. after the elimination of the (fast) bath degrees of freedom and the discretization of the chemical state variables.

${ }^{3}$ More precisely: the compound motor-filament system, see below.

${ }^{4}$ Trivial neutral translational and rotational degrees of freedom are assumed to have been eliminated already.
} 
bath $92,93,185$. As a result, a renormalization of the potential, the noise, the dissipation mechanism etc. in the stochastic dynamics of the "intrinsic reaction coordinate" will arise, but the main point is that finally an effective description of the entire reaction in terms of a single generalized coordinate (also called collective coordinate, state variable or reaction coordinate) can been achieved, see also section 3.4.1.

In the case of complex biomolecules such as a motor enzyme, different possible paths between the various metastable states may be realized with non-negligible probability [579, 594. In such a case, more than one collective coordinate (state variable) has to be kept in order to admit a faithful representation of all the possible pathways in the reduced description. Moreover, only some of those state variables can be identified with chemical reaction coordinates, while others are of a more mechanical or geometrical nature (see below). Finally, these concepts can also be generalized to cases without a clear cut distinction of metastable states and rare transition events, i.e. some of the (non-chemical) state variables may be governed by a predominantly relaxational or diffusive dynamics.

Often, an equivalent way to discriminate relevant (generalized) coordinates which should be explicitly kept from "irrelevant noise" which can be savely eliminated is according to their characteristic time scale [93, 150, 186] (see also section 3.4.1): On the smallest time scales (femtoseconds) the motion of the molecule consists of fast but small fluctuations, while significant conformational changes will develop only on a much slower time scale of milliseconds.

\subsubsection{Mechanical and chemical state variables}

For realistic systems, the above program - starting with the full quantum mechanical problem and ending with a simple approximate dynamics in terms of a few relevant classical stochastic variables - cannot be practically carried out. Therefore, a phenomenological modeling, roughly based on the above intuitive picture and supplemented by experimental evidence is necessary, see also section 3.4.1. In the case of a motor enzyme like kinesin, the picture one has in mind is the following: The actual chemical conversion of ATP into ADP and $\mathrm{P}_{i}$ takes place in relatively well defined and small regions of the enzyme - the ATP binding pockets of the two heads. This chemical cycle is captured by a set of chemical reaction coordinates or state variables $y$. On the other hand, the much larger conformational changes of the enzyme as a whole are represented by a different set of "mechanical" pollective coordinates or state variables $x$. Note that both $y$ and $x$ are ultimately describing nothing else than the geometrical configuration of the nuclei, but the distinction between chemical and mechanical coordinates are both conventional and suggestive ${ }^{5}$.

Particularly difficult to explicitly derive from first principles is the central feature of the enzymatic chemical reaction cycle, namely that reactant and product molecules can be exchanged with the environment. Typically, such events are possible (with non-negligible probability) only in certain specific configurations of the enzyme and

\footnotetext{
5 "Mechanical" may refer here either to the fact that $x$ represents the global geometrical shape of the molecule, or to the fact that some mechanical "strains" in the molecule, which have been created by the chemical transitions, may be released through a relaxational dynamics of $x$.

${ }^{6}$ Note that the same applies for the (already eliminated) "irrelevant" degrees of freedom both of the environment and of the molecular motor itself: The dissipation and fluctuation effects, to which they give rise, may be either due to "mechanical" processes (vibrations, elasic and/or inselastic collisions etc.) or due to chemical processes (making and braking of chemical bonds etc.).
} 
it is assumed that the collective coordinates $(x, y)$ are capable to faithfully monitor such events and, in particular, of whether some reactant/product molecule is presently attached to one of the heads or not?. The binding probabilities for both reactants and products depend on their concentrations in the environment of the enzyme. The fact that these dependences should be simply proportional to the respective concentrations is very suggestive and we will take it for granted in the following without any further derivation from a more fundamental description.

It is quite plausible that whether or not one or both heads of the kinesin are attached to the microtubulus will have a significant influence on both, the chemical reaction process and the mechanical behavior [525,587]. A priori, we should therefore not speak of an isolated kinesin but rather of the compound kinesin-microtubulus system. However, similar as for the previously discussed attachment and detachment of reactants and products, the attachment and detachment of the heads as well as the influence of the microtubulus in the attached state can be represented by the relevant collective coordinates $(x, y)$ of the motor enzyme alone, if they have been appropriately chosen.

\subsubsection{Discrete chemical states}

We recall that the "mechanical coordinates" $x$ describe configurational changes of the enzyme as a whole, while the actual chemical ATPase is monitored by the "chemical coordinates" $y$ and takes place in the rather restricted spatial regions of the ATP binding pockets. One therefore expects that transitions between different "chemical states" $y$ are accomplished during rather short time intervals in comparison with the typical time scales on which the global geometrical configuration $x$ notably changes [525]. As a consequence, one can neglect the details of the transitions between chemical states itself and focus on a discrete number of states, $m=1,2, \ldots M_{\text {tot }}$, with certain "instantaneous" transition rates $k_{m \rightarrow m^{\prime}}(x)$ between them, which in general still depend on the configuration $x$. Similarly, the potential landscape, which $x$ experiences, still depends on the "chemical state" $m$. Formally, the transition rates $k_{m \rightarrow m^{\prime}}(x)$ are those between the local minima with respect to the $y$-coordinates at fixed $x$, which, however, need not necessarily be local minima in the full $x-y$-space.

In doing so, it is taken for granted that a well-defined, relatively samll number $M_{\text {tot }}$ of discrete "chemical states" exists and that all transitions between them can be described in terms of rates $k_{m \rightarrow m^{\prime}}(x)$. Though such an approach is known to be problematic in other types of proteins due to their general "glass-like" properties and especially for the binding and unbinding processes of reactants and products in "pockets" of the proteins [595,596], in the context of motor enzymes like kinesin it has to our knowledge not been theoretically or experimentally challenged so far and we will therefore follow the general belief in its adequacy.

At this point it should be emphasized again that one motor enzyme incorporates two "heads", each endowed with an ATP-binding pocket and able to loop through its own chemical reaction cycle. Thus the set of "chemical coordinates" (vectors) $y$ is in fact composed of two subsets (scalars), $y=\left(y_{1}, y_{2}\right)$, one for each head, and similarly

\footnotetext{
${ }^{7}$ It is indeed plausible that the set of possible geometrical shapes of the enzyme while a reactant molecule is bound will be satisfactorily disjoint from the corresponding set in the absence of the reactant, and similarly for the products, provided the coordinates $(x, y)$ have been suitably chosen.
} 
the discretized states are of the form

$$
m=\left(m_{1}, m_{2}\right), m_{1,2} \in\{1,2, \ldots, M\} .
$$

For instance, for the standard model for the ATP reaction cycle consisting of $M=4$ distinct states (cf. section 7.2.1), the compound set of states $m$ will comprise $M_{\text {tot }}=$ $M^{2}=16$ elements. Note that exactly simultaneous reaction steps in both heads have negligible probability, i.e., only indices $m=\left(m_{1}, m_{2}\right)$ with $m^{\prime}=\left(m_{1}^{\prime}, m_{2}\right)$ or $m^{\prime}=\left(m_{1}, m_{2}^{\prime}\right)$ are possible in $k_{m \rightarrow m^{\prime}}(x)$. In order to further reduce the number of nontrivial transition rates $k_{m \rightarrow m^{\prime}}(x)$, one common and suggestive assumption [525] is that only transitions between "neighboring" states within either of the two chemical cycles occur with non-negligible probability, i.e.,

$$
k_{m \rightarrow m^{\prime}}(x)=0 \text { if } m^{\prime} \notin\left\{\left(m_{1} \pm 1, m_{2}\right),\left(m_{1}, m_{2} \pm 1\right)\right\},
$$

where states $m_{1}$ which differ by a multiple of $M$ are identified, and similarly for $m_{2}$. In other words, each of the two chemical reaction cycles loops through a definite sequence of states, bifurcations into different chemical pathways are ruled out.

Note that the cooperativity between the two heads, mentioned at the end of section 7.1, is mediated by the geometrical configuration $x$ and will manifest itself in the $x$ dependence of the rates, possibly reducing the number of non-trivial transition rates (7.2) once again.

\subsection{Simplified stochastic model}

While the so far reasoning and approximations have been relatively systematic and microscopically well founded, further possible simplifications are necessarily of a more drastic and phenomenological nature.

In concrete models, the geometrical configuration of the motor is usually assumed to be characterized by a singl \& $^{8}$ relevant state variable $x$. One convenient choice for $x$ turns out to be the position of the molecular motor along the microtubulus. To be precise, $x$ may for instance be chosen to represent the position of the common center of mass of the two heads. Indeed, knowing that the motor enzyme walks in a stepby-step fashion straight along the $\beta$-tubulin sites of one and the same protofilament, it is suggestive that the geometrical configuration of each of the two heads can be reconstructed quite faithfully from the knowledge of the position $x$ (the rest of the motor molecule ("tail" and "middle segment", cf. section 7.1) does not seem to play a significant role for the actual "motor function").

Once the relevant collective chemical and mechanical state variables have been identified along the above line of reasoning, their "thermal environment" consits of two parts (cf. section 7.2.1): Namely, on the one hand there are the huge number of "irrelevant" degrees of freedom of the liquid which surrounds the protein, and on the other hand there are those of the protein itself and of the microtubulus with which it interacts. Upon eliminating them along the lines of section 3.4.1, their effects on the discretized chemical state variables are captured by the phenomenological rates (7.2). However, their effects on the mechanical state variable $x$ are more involved

\footnotetext{
${ }^{8}$ In other words, each "head" has its own (discrete) chemical state variable (cf. equation (7.1)), but the geometrical shape of the entire motor (including the two "heads") is described by a single (continuous) state variable $x$.
} 
due to the fact that $x$ does not simply represent the cartesian coordinate of a point particle but rather the complicated geometrical configurations of the entire motor protein and in this sense is a generalized coordinate. As a consequence, the so-called solvent friction, caused by the eliminated degrees of freedom of the surrounding fluid, comprises not only a Stokes-type viscous friction against straigh translational motion but also a damping force against configurational changes of the geometrical enzyme structure. Similarly, the so-called protein friction [9,525,597, caused by the eliminated degrees of freedom of the enzyme and the microtubulus, is composed of two analogous partial effects: on the one hand, a viscous drag against straight translational motion due to the continuous making and breaking of bonds between the motor and the microtubulus; on the other hand an effective "internal" frictional force against changes of the geometrical configuration. All these friction mechanisms are in general not invariant under arbitrary translations of $x$ and are therefore explicitly $x$-dependent? The same carries over to the thermal fluctuations which they bring along ("solvent noise" and "protein noise"), see also section 3.4.1. Since quantitatively the effects of protein friction are typically comparable or even more important than those of solvent friction [9,525], a quite significant spatial inhomogeneity of the friction and the thermal noise is expected [560]. We recall that the microscopic origin of both solvent and protein friction is partly of a mechanical (geometrical) nature (mainly collisions and vibrations, respectively) and partly due to the making and braking of numerous weak chemical bonds, as detailed in section 7.2.1. On an even more basic level, all these distinctions become again blurred since the ultimate origin of friction is always the "roughness" of some effective potential energy landscape.

\subsubsection{Stochastic ratchet dynamics}

On the basis of the above considerations, the simplest working model for the stochastic dynamics governing the mechanical coordinate (position) $x(t)$ is of the form

$$
\eta \dot{x}(t)=-V_{m}^{\prime}(x(t))+F+\xi(t)
$$

where $m=m(t)$ is understood as a stochastic process, with states (7.1) and transition rates $k_{m \rightarrow m^{\prime}}(x)$. The assumption of a first order (overdamped) dynamics in time is justified as usual by the fact that on these small scales inertia effects can be safely neglected [186]. The damping coefficient $\eta$ and the random noise $\xi(t)$ model the effects of the environment and of the eliminated fast degrees of freedom of the molecular motor itself (possibly also of the microtubulus) and both these contributions are treated as a single thermal bath. Under the assumption that the origin of $\xi(t)$ is a very large number of very fast processes (on the time scale of $x$ ) we can model those fluctuations as a Gaussian noise of zero mean and negligible correlation time

$$
\langle\xi(t) \xi(s)\rangle=2 \eta k_{B} T \delta(t-s) .
$$

In fact, already the very form of the dissipation assumed on the left hand side of (7.3) leaves no other choice for the noise $\xi(t)$ at equilibrium, see section 3.4.1. A further

\footnotetext{
${ }^{9}$ Regarding the Stokes-type viscous friction, we recall that $x$ represents not only the position but also the changing geometrical shape of the motor molecule. As mentioned in section 6.4.2 we furthermore expect corrections of Stokes friction due to the nearby microtubulus, which are again in general $x$ dependent.
} 
assumption implicit in (7.3) is the independence of the coupling to the heat bath $\eta$ (see below (2.5)) from the chemical state $m$ and the geometrical configuration $x$. The former simplification is plausible in view of the fact that the chemical processes only involve a very restricted region of the entire motor enzyme. On the other hand, the $x$-independence of $\eta$ is not obvious in view of our above considerations about solvent and protein friction, but can be justified as follows: First, inhomogeneous friction, and in particular protein friction, can be modeled quite well by means of potentials $V_{m}(x)$ in (7.3) with a suitably chosen "roughness" on a very "fine" spatial scale. After a spatial coarse graining, only the broader structures of the potential survive while the initially homogeneous "bare" friction is dressed by an inhomogeneous renormalization contribution. A second possibility consists in a change of variable ${ }^{\mathbb{T}}$ as detailed in section 6.4.2.

Note that $\eta$ accounts for the coupling of the thermal environment (fast degree of freedom) of the molecular motor only. The additional slow variable representing the cargo of the motor can be accounted for [587] via a contribution of the form $-\langle\dot{x}\rangle \eta_{\text {cargo }}$ to the force $F$ in (7.3) under the tacit but apparently realistic assumption that its connection to the motor (via "tail" and middle segment", cf. section 7.1) is sufficiently elastic [511,512,570]. Although the cargo is typically much bigger than the motor itself, this viscous drag force seems negligibly small [587] in comparison with the intrinsic friction of the motor, modeled by $\eta \dot{x}(t)$ in (7.3).

The deterministic mechanical forces in (7.3) on the one hand derive from an effective, free-energy like potential $V_{m}(x)$ and on the other hand leave room for the possibility of an externally applied extra force $F$. Originating from the potential energy landscape in which the nuclei of the motor and its environment move, the effective (renormalized) potential $V_{m}(x)$ in addition accounts for some of the effects of the eliminated fast degrees of freedom. The approximate independence of this effective potential $V_{m}(x)$ from the external load $F$ is assumed here for the sake of simplicity [559,598 600]. On the other hand, the dependence of the potential on the chemical state $m$ is crucial. The latter in conjunction with the $x$-dependence of the chemical reaction rates $k_{m \rightarrow m^{\prime}}(x)$ is called the mechanochemical coupling mechanism of the model motor enzyme, decisive for the chemical to mechanical energy transduction [2]. The underlying picture is that certain chemical reaction steps take place preferably or even exclusively while the molecular motor has a specific geometrical shape $x$. In turn, certain mechanical relaxations of strains or thermally activated configurational transitions may be triggered or made possible only after a certain chemical reaction step has been accomplished.

Clearly, the dynamical behavior of the motor enzyme is invariant after a step of one head has been completed if at the same time the chemical states $m_{1}, m_{2}$ of the two heads are exchanged [143, 601]. This invariance under a displacement $x \mapsto x+L$

\footnotetext{
${ }^{10}$ In this case, the transformed potentials in 7.3 remain periodic but in general pick up an $F$ dependence, which we neglect for the sake of simplicity (see below).

${ }^{11}$ As far as $x$ describes the center of mass of the molecular motor, the simple $F$-dependence on the right hand side of 7.3 is fully justified. However, in so far as $x$ at the same time accounts for the geometrical shape of the motor molecule, the relation between position and shape and hence the effective potentials $V_{m}(x)$ are expected to change upon application of a force $F$.

${ }^{12}$ The $F$-independence of the rates $k_{m \rightarrow m^{\prime}}(x)$ (and a forteriori of the number $M_{\text {tot }}$ of chemical states) is plausible on the basis of the physical picture from section 7.2.3 (the chemical processes are spatially localized and thus involve negligibly small changes of the geometrical configuration of the motor molecule).
} 
and simultaneously $\left(m_{1}, m_{2}\right) \mapsto\left(m_{2}, m_{1}\right)$ has to be respected by the potentials $V_{m}(x)$ and the rates $k_{m \rightarrow m^{\prime}}(x)$,

$$
\begin{aligned}
& V_{m}(x+L)=V_{\bar{m}}(x) \\
& k_{m \rightarrow m^{\prime}}(x+L)=k_{\left(\bar{m} \rightarrow \overline{m^{\prime}}\right)}(x),
\end{aligned}
$$

where the bar denotes the exchange of the vector-components:

$$
\overline{\left(m_{1}, m_{2}\right)}:=\left(m_{2}, m_{1}\right) \text {, }
$$

and where the spatial period $L$ is given by the length of one tubulin dimer (about $8 \mathrm{~nm}$ ). Consequently, the functions $V_{m}(x)$ and $k_{m \rightarrow m^{\prime}}(x)$ are invariant under $x \mapsto x+2 L$ without any change of the chemical states.

The polarity of the microtubulus, on which the motor walks, reflects itself in a generic spatial asymmetry of the potential $V_{m}(x)$ as well as of the rates $k_{m \rightarrow m^{\prime}}(x)$. Note that on top of that, there is also an intrinsic asymmetry of the motor domains (but not of the entire enzyme, see section 7.1): If one detaches a motor domain from the microtubulus, turns it around by $180^{\circ}$, and puts it back on the microtubulus, no invariance arises 143,582,583, that is, reflection symmetry is broken. In other words, the asymmetry of the microtubulus is necessary to make manifest the asymmetry of the motor, while the asymmetry of the compound system is caused and maybe even mutually enhanced by both [17, 602 .

The stochastic dynamics (7.3) as it stands is a convenient starting point for numerical simulations (cf. section 2.2) but not for quantitative analytical calculations. Exactly like for the fluctuating potential ratchet model in equations (4.12), (4.13), one obtains the following master equation (reaction-diffusion equation) equivalent to (7.3):

$$
\begin{aligned}
\frac{\partial}{\partial t} P_{m}(x, t) & =\frac{\partial}{\partial x} \frac{\left[V_{m}^{\prime}(x)-F\right] P_{m}(x, t)}{\eta}+\frac{k_{B} T}{\eta} \frac{\partial^{2}}{\partial x^{2}} P_{m}(x, t) \\
& -P_{m}(x, t) \sum_{m^{\prime}} k_{m \rightarrow m^{\prime}}(x)+\sum_{m^{\prime}} P_{m^{\prime}}(x, t) k_{m^{\prime} \rightarrow m}(x),
\end{aligned}
$$

where $P_{m}(x, t)$ is the joint probability density that at time $t$ the chemical state is $m$ and the motor enzyme is at the position $x$, with normalization $\sum_{m} \int d x P_{m}(x, t)=1$. In order to technically simplify matters one defines similarly as in (2.22) reduced densities

$$
\hat{P}_{m}(x, t):=\frac{1}{2} \sum_{n=-\infty}^{\infty}\left\{P_{m}(x+2 n L, t)+P_{\bar{m}}(x+2(n+1) L, t)\right\} .
$$

The reduced densities satisfy the same master equation (7.8) but are periodic in $x$ with period $2 L$ and normalization

$$
\sum_{m} \int_{0}^{2 L} d x \hat{P}_{m}(x, t)=1
$$

The symmetries (7.5), (7.6) furthermore imply that

$$
\hat{P}_{m}(x, t)=P_{\bar{m}}(x+L, t)=\hat{P}_{m}(x+2 L, t) .
$$


Once $\hat{P}_{m}(x, t)$ is determined, the average speed of the motor enzyme follows along the same line of reasoning as in section 2.3 as ${ }^{13}$

$$
\langle\dot{x}\rangle=\frac{1}{\eta}\left[F-\sum_{m} \int_{0}^{2 L} d x V_{m}^{\prime}(x) \hat{P}_{m}(x, t)\right] .
$$

A further interesting quantity is the rate $r_{A T P}=r_{A T P}(t)$ of ATP-consumption per time unit, given by

$$
r_{A T P}=\sum_{m, m^{\prime}} \chi_{m, m^{\prime}}^{A T P} \int_{0}^{2 L} d x\left\{\hat{P}_{m}(x, t) k_{m \rightarrow m^{\prime}}(x)-\hat{P}_{m^{\prime}}(x, t) k_{m^{\prime} \rightarrow m}(x)\right\}
$$

where $\chi_{m, m^{\prime}}^{A T P}$ is the indicator function for ATP-binding transitions $m \rightarrow m^{\prime}$. For example, using the labeling of the chemical states from section 7.2.1 for the standard ATP-hydrolysis cycle with $M=4$ states, we have

$$
\chi_{m, m^{\prime}}^{A T P}=\left\{\begin{array}{cccc}
1 & \text { if } & m=\left(1, m_{2}\right) & \text { and } m^{\prime}=\left(2, m_{2}\right) \\
1 & \text { if } & m=\left(m_{1}, 1\right) & \text { and } m^{\prime}=\left(m_{1}, 2\right) \\
0 & \text { otherwise } & &
\end{array}\right.
$$

A comparison of the above model setup with the working model from section 3.1 very obviously establishes a close connection between our present section about molecular motors and the general framework for our studies of ratchet models, especially the class of pulsating ratchets 5 according to the classification scheme from section 3.3 . However, there is also one important point in which the present model goes beyond the latter general framework. Namely, there is a back-coupling of the state-variable $x(t)$ to the "potential fluctuations" $m(t)$ through the $x$-dependence of the transition rates $k_{m \rightarrow m^{\prime}}(x)$. Especially, the statistical properties of the potential fluctuations $m(t)$ can no longer be assumed a priori as stationary. We will show later in section 7.4 .2 that far away from equilibrium an effective $x$-independence of the potential fluctuations $m(t)$ may arise nevertheless, entailing stationarity of their statistical properties in the long time limit, i.e. a veritable pulsating ratchet scheme is recovered.

\subsubsection{Nonequilibrium chemical reaction}

At thermal equilibrium, the concentrations of ATP, ADP, and $\mathrm{P}_{i}$ are not independent, their ratio $C_{A T P}^{0} / C_{A D P}^{0} C_{P_{i}}^{0}$ satisfies the so-called mass action law. Especially, the numerical value of this ratio must be independent of whether any motor enzymes (acting as catalyst) are present or not. Since this represents a single constraint for three variables, there still remains a freedom in the choice of two out of the three equilibrium

\footnotetext{
${ }^{13}$ We recall that the argument $t$ in $\langle\dot{x}\rangle$ is omitted (cf. (3.4) since in most cases one is interested in the steady state behavior with $\hat{P}_{m}(x, t)=\hat{P}_{m}^{s t}(x)$. The same applies for the rate $r_{A T P}=r_{A T P}(t)$ in (7.13).

${ }^{14}$ The driving $f(t)$ of the pulsating potential $V(x, f(t))$ is denoted here by $m(t)$ and the pulsating potential itself by $V_{m}(x)=V_{m(t)}(x)$ (cf. (3.1) and below (7.3), respectively). Moreover, $m(t)$ is here a discrete and - in general - two-dimensional state variable (cf. (7.1)), though in most concrete models (see sections 7.4 7.6) again a simplified, effectively one-dimensional description will be adopted.

${ }^{15}$ It may be worth to recall that for traveling potential ratchets and their descendants (sections 4.4 and 4.5) a broken symmetry of the potential is not necessary for directed transport, though for real molecular motors this symmetry will be typically broken.
} 
concentrations $C_{A T P}^{0}, C_{A D P}^{0}$, and $C_{P_{i}}^{0}$. We consider an arbitrary but fixed such choice from now on. Since the system is an equilibrium system, the stochastic dynamics has furthermore to respect the so-called condition of detailed balance [98 101, 148, 152]. For our specific model (7.3) this condition can be readily shown to imply the following relation between the transition rates $k_{m \rightarrow m^{\prime}}(x)$ and the corresponding potentials $V_{m}(x)$ and $V_{m^{\prime}}(x)$ for any pair of chemical states $m$ and $m^{\prime}$ :

$$
\frac{k_{m \rightarrow m^{\prime}}(x)}{k_{m^{\prime} \rightarrow m}(x)}=\exp \left\{\frac{V_{m}(x)-V_{m^{\prime}}(x)}{k_{B} T}\right\} .
$$

Thus, one of the two rates in (7.15) can be considered as a free, phenomenological function of the model, while the other rate is then fixed. Note that the appearance of negligibly small rates in (7.2) as well as the symmetry relations (7.5), (7.6) are still compatible with (7.15).

The salient point is now to clarify what is meant by saying that one goes "away from equilibrium" in our present context. Meant is, that as far as the heat bath properties of the environment (random fluctuations and energy dissipation mechanism) are concerned, nothing is changed as compared to the thermal equilibrium case. The only things which change are the concentrations of reactants and/or products 603.

For instance, if the ATP concentration $C_{A T P}$ is changed away from its equilibrium value $C_{A T P}^{0}$, then all the rates $k_{m \rightarrow m^{\prime}}(x)$ remain unchanged except those which describe the binding of ATP to one of the two heads of the molecular motor. As discussed in section 7.2 .2 these rates simply acquire an extra multiplicative factor of the form $C_{A T P} / C_{A T P}^{0}$, i.e., (7.15) is generalized

$$
\frac{k_{m \rightarrow m^{\prime}}(x)}{k_{m^{\prime} \rightarrow m}(x)}=\left[1+\left(\frac{C_{A T P}}{C_{A T P}^{0}}-1\right) \chi_{m, m^{\prime}}^{A T P}\right] \exp \left\{\frac{V_{m}(x)-V_{m^{\prime}}(x)}{k_{B} T}\right\},
$$

where $\chi_{m, m^{\prime}}^{A T P}$ is the ATP-binding indicator function from (7.14). Similar modifications arise if the concentrations of $\mathrm{ADP}$ and $\mathrm{P}_{i}$ are changed. However, in order to describe the real situation one may without loss of generality assume that these concentrations have already their correct value due to our choice of $C_{A D P}^{0}$ and $C_{P_{i}}^{0}$. In doing so, it follows from the quantitative biological findings mentioned in section 7.1 that $C_{A T P}$ has to be chosen about 6 decades beyond its equilibrium value $C_{A T P}^{0}$ :

$$
\frac{C_{A T P}}{C_{A T P}^{0}} \simeq 10^{6}
$$

From the conceptual viewpoint we are thus facing the following interesting setup of a far from equilibrium system: On one hand, the system is in contact with a thermal equilibrium heat reservoir as far as dissipation and fluctuations are concerned. On the other hand, it is in contact with several reservoirs of reactant and products with concentrations which are externally kept far away from equilibrium. All these various reservoirs are physically localized at the same place but the effects due to their direct interaction with each other is practically negligible. Only the indirect interaction by way of the motor molecules (catalysts) is relevant.

\footnotetext{
${ }^{16}$ Without discretizing the chemical state variable(s) (or equivalently, assuming that a separation of time-scales exists such that a rate description is justified) the proper reformulation of a relation like in (7.16) does not seem possible, see also section 4.6 and 186 .
} 


\subsection{Collective one-head models}

At this stage, the number of free, phenomenological functions in $(7.8)$ is still very large. There is little chance to make a convincing guess for each of them on the basis of our present knowledge about the structure and functioning of the real motor enzyme, while for fitting the dynamical behavior of the model to experimental curves, the available variety and accuracy of measurements is not sufficient. Our next goal must therefore be to reduce the effective number $M_{\text {tot }}$ of relevant chemical states.

\subsubsection{A.F. Huxley's model}

The most prominent such simplification goes back to A.F. Huxley's 1957 paper [4] and consists in the assumption of one instead of two heads per motor enzyme. In our model (7.3) this means that $m$ is no longer composed of two "substates", see equation (7.1), but rather is a scalar state variable with $M_{\text {tot }}=M$ values

$$
m \in\{1,2, \ldots, M\} .
$$

Likewise, $x$ now represents the center of mass of a single head. As a consequence, the symmetries (7.5), (7.6) become

$$
V_{m}(x+L)=V_{m}(x), \quad k_{m \rightarrow m^{\prime}}(x+L)=k_{m \rightarrow m^{\prime}}(x)
$$

and equations (7.9)-(7.11) are replaced by

$$
\begin{aligned}
& \hat{P}_{m}(x, t):=\sum_{n=-\infty}^{\infty} P_{m}(x+n L, t) \\
& \hat{P}_{m}(x+L, t)=\hat{P}_{m}(x, t) \\
& \sum_{m=1}^{M} \int_{0}^{L} d x \hat{P}_{m}(x, t)=1 .
\end{aligned}
$$

Furthermore, equations (7.12), (7.13) assume the form

$$
\begin{aligned}
& \langle\dot{x}\rangle=\frac{1}{\eta}\left[F-\sum_{m=1}^{M} \int_{0}^{L} d x V_{m}^{\prime}(x) \hat{P}_{m}(x, t)\right] \\
& r_{A T P}=\int_{0}^{L} d x\left\{\hat{P}_{1}(x, t) k_{1 \rightarrow 2}(x)-\hat{P}_{2}(x, t) k_{2 \rightarrow 1}(x)\right\} .
\end{aligned}
$$

Finally, all rates $k_{m \rightarrow m^{\prime}}(x)$ with $m^{\prime} \neq m \pm 1$ are zero according to (7.2), and for $m^{\prime}=m \pm 1$ equation (7.16) takes the form

$$
\frac{k_{m \rightarrow m^{\prime}}(x)}{k_{m^{\prime} \rightarrow m}(x)}=\left[1+\left(\frac{C_{A T P}}{C_{A T P}^{0}}-1\right) \delta_{m, 1} \delta_{m^{\prime}, 2}\right] \exp \left\{\frac{V_{m}(x)-V_{m^{\prime}}(x)}{k_{B} T}\right\}
$$

where the ATP binding transition is assumed to be $m=1 \rightarrow m^{\prime}=2$ and where states which differ by a multiple of $M$ are identified.

A second ingredient of Huxley's model is a "backbone" to which a number $N$ of such single headed motors is permanently attached. The emerging intuitive picture is a centipede, walking along the polymer filament. The interaction of the single-headed 
motors is mediated by the common backbone, assumed rigid and moving with constant speed $\langle\dot{x}\rangle$, but otherwise they are considered as operating independently of each other. We may then concentrate on any of the single heads and without loss of generality denote the site where this specific head is rooted in the backbone by $\langle\dot{x}\rangle t$. In physical terms, we are dealing with a mean field model $(N \rightarrow \infty)$, described by an arbitrary but fixed reference head according to (7.3), where the potentials $V_{m}(x)$ and the rates $k_{m \rightarrow m^{\prime}}(x)$ may, in general, acquire an additional dependence on the backbone site $\langle\dot{x}\rangle t$. The possible difference between the center of mass of the head $x$ and the point $\langle\dot{x}\rangle t$ where it is attached to the backbone may, for instance, reflect a variable angle between the head's length axis and the polymer filament, similarly to a human leg while walking. Thus, we may also look upon $\langle\dot{x}\rangle t$ as an additional relevant (slow) mechanical state variable of the motor. However, no extra equation of motion for this coordinate is needed since it already follows in the spirit of a mean field approach from the behavior of the other relevant mechanical state variable $x$. For instance, a term of the form $\kappa(x-\langle\dot{x}\rangle t)^{2}$ in the potentials $V_{m}(x)$ models a harmonic coupling of the head to the uniformly advancing backbone, with spring constant $\kappa$. As in any mean field model, the characteristic feature is the appearance of an a priori unknown "order parameter", $\langle\dot{x}\rangle$ in our case, which has to be determined self-consistently in the course of the solution of the model (7.3), (7.12) (for an explicit example see (7.28), (7.29) below). We emphasize that for a rigid backbone, in the limit $N \rightarrow \infty$ Huxley's mean field approach is not an approximation but rather an exact description because the interaction between the single motors is of infinite range.

We remark that such a model of $N$ single headed motors with a mean field coupling through a rigid "backbone" may even be acceptable as a rough approximation in the case of a single kinesin molecule. Admittedly, the number $N=2$ of involved heads makes a mean field approximation somewhat questionable. On the other hand, taking into account that a rather "heavy" load is attached to the motor, may render the assumption of an uniformly moving backbone not so bad [587. On top of that, the cooperativity of the two heads in the real kinesin is at least roughly incorporated into the model through their interaction via the backbone and through the implicit assumption that the motor will not diffuse away from the microtubulus even if both heads happen to take a step at the same time.

More suggestive is the case when an appreciable number $N$ of single motor molecules truly cooperate. This may be a couple of kinesins which drag a common "big" cargo. More importantly, there exist motor enzymes different from kinesin which indeed are interconnected by a backbone-like structure by nature. Examples are so-called myosin enzymes, walking on polymer filament tracks called actin, thereby not carrying loads but rather playing a central role in muscular contraction [346, 568, 576]. While the quantitative and structural details are different from the kinesin-microtubulus system, the main qualitative features of the myosin-actin system are sufficiently similar [582, 604, 605] such that the same general framework $(7.2)-(7.12)$ is equally appropriate in both cases $\square$. Though a single myosin enzyme again consists of two individual motor domains, their cooperativity seems not so highly developed as for kinesin [582, 607] and therefore the above mentioned mean field approximation for a large number $N$ of interacting single heads appears indeed quite convincing.

\footnotetext{
${ }^{17}$ Intriguingly enough, certain species of the myosin superfamily (e.g. the so-called myosin V subfamily) show again a behavior similar to kinesin [568, 506]. In the following we always have in mind collectively operating myosin species (the myosin II subfamily).
} 
In his landmark paper 迎, Huxley proposed a model of this type without any knowledge about the structural features of an individual motor enzyme! It is not difficult to map the slightly different language used in his model to our present framework, but since the details of his setting cannot be upheld in view of later experimental findings, we desist from explicitly carrying out this mapping here. While the model is apparently in satisfactory agreement with the main experimental facts available at that time, Huxley himself points out [4 that "there is little doubt that equally good agreement could be reached on very different sets of assumptions, all equally consistent with the structural, physical and chemical data to which this set has been fitted. The agreement does however show that this type of mechanism deserves to be seriously considered and that it is worth looking for direct evidence of the side pieces."

\subsubsection{Free choice of chemical reaction rates}

One specific point of Huxley's model is worth a more detailed discussion since it illustrates a much more general line of reasoning in the construction of such models. In doing so, we first recall that we are dealing with a single head motor model described by $M=4$ (scalar) chemical states $m$ : 1 . the head without anything bound to it; 2 . the head with an ATP bound; 3 . the head with an ADP and a $\mathrm{P}_{i}$ bound; 4 . the head with an ADP bound. The chemical state variable travels back and forth between neighboring states of this cycle according to the transition rates $k_{m \rightarrow m^{\prime}}(x)$, respecting (7.25) if $m^{\prime}=m \pm 1$ and being zero otherwise. For the case of kinesin, we have discussed at the beginning of section 7.1 in addition the "affinity" between head and filament in each state, which essentially tells us whether the head is attached to the filament or not in the respective state, and which has to be taken into account in the concrete choice of the respective model potentials $V_{m}(x)$. We remark that this correspondence between states and affinity is somewhat different for myosin [9] and again different in Huxley's model, but will not play any role in the following, since it only regards quantitative, but not qualitative properties of the potential $V_{m}(x)$.

As a first simplification, Huxley postulates a 3 -state model, in which $m=2$ and $m=3$ in our above scheme are treated as a single state, and the question arises of whether and how this can be justified, at least in principle. One possible line of reasoning goes as follows: Aiming at a unification of $m=2$ and $m=3$ means in particular that we should choose $V_{2}(x)=V_{3}(x)$ and thus $k_{2 \rightarrow 3}(x)=k_{3 \rightarrow 2}(x)$ according to (7.25). Since there are no further a priori restrictions on the choice of these rates, we may take them as independent of $x$ and very large 18 . Thus, as soon as the system reaches either state 2 or 3 it will be practically instantaneously distributed among both states with equal probability. One readily sees that the two states can now be treated as a single "superstate" if the two transition rates out of this state are defined as half the corresponding original values $k_{2 \rightarrow 1}(x)$ and $k_{3 \rightarrow 4}(x)$. At first glance, it may seem that in this reduced 3-state model, the condition (7.25), in the case that $m$ represents the new "superstate", has to be modified by a factor $1 / 2$. However, since in the stochastic dynamics (7.3) only the derivative of the potential $V_{m}(x)$ appears, this factor $1 / 2$ can be readily absorbed into an additive constant of that potential.

Given the reduced model with $M=3$ states, Huxley furthermore assumes that the 3 "forward" rates $k_{m \rightarrow m+1}(x)$ can be freely chosen, while the 3 "backward" rates

\footnotetext{
${ }^{18}$ Such a choice is obviously admissible within our general modeling framework; how to justify it against experimental findings is a different matter 579,608610 .
} 
$k_{m+1 \rightarrow m}(x)$ are negligibly small. On the other hand, equation (7.25) tells us that the 3 forward rates can indeed be chosen freely, but once they are fixed, the 3 backwards rates are also fixed. At this point, one may exploit once again the observation that only the derivatives $V_{m}^{\prime}(x)$ enter the dynamics (7.3) and therefore we still can add an arbitrary constant to any of the three model potentials $V_{m}(x)$. Under the additional assumption that $\exp \left\{\left[V_{m}(x)-V_{m+1}(x)\right] / k_{B} T\right\}$ varies over one spatial period $L$ at most by a factor significantly smaller than $\left(C_{A T P} / C_{A T P}^{0}\right)^{1 / 3} \simeq 10^{2}$ (see 7.17), one readily sees that by adding appropriate constants to the 3 potentials $V_{m}(x)$ one can make the ratios $k_{m \rightarrow m+1}(x) / k_{m+1 \rightarrow m}(x)$ rather small for all 3 values of $m$ according to (7.25). Pictorially speaking, by adding proper constants to the potentials $V_{m}(x)$ one can split and re-distribute the factor $C_{A T P} / C_{A T P}^{0}$ from (7.17) along the entire chemical reaction cycle. In this way, all 3 backward rates $k_{m+1 \rightarrow m}(x)$, though not exactly zero, can indeed be practically neglected. Generalizations to more than 3 states and to the neglection of only some, but not all, backward rates are obvious.

A final important observation concerns the case $M=3$ with all the forward rates still at our disposition and all the backward rates approximately neglected. Specifically, one may assume that $V_{2}^{\prime}(x)=V_{3}^{\prime}(x)$ and that the corresponding forward rate $k_{2 \rightarrow 3}$ is $x$-independent and very large. The two states $m=2$ and $m=3$ can then again be lumped into a single superstate. The result is [16] a model (7.3) with $M=2$ effective chemical states but with both, the forward and backward rates between these two states, still free to choose.

We have thus achieved by way of various simplifying assumptions our goal to substantially reduce the number of free, phenomenological functions in the model (7.3). Still, even for the minimal number $M=2$ of chemical states the shape of the two potentials and especially the choice of the two rates [9, 520, 587, 601 are very difficult to satisfactory justify on the basis of experimental findings. Accordingly, the existing literature does not seem to indicate that a common denominator of how these functions should be realistically chosen is within hands reach.

\subsubsection{Generalizations}

Huxley's choice of model parameters and functions (7.18), (7.19) in the general setup (7.3) has been subsequently modified and extended in various ways in order to maintain agreement with new experimental findings. Most of the following works include verifications of the theoretical models against measurements, though we will not repeat this fact each time. Moreover, a detailed discussion of the specific choices and justifications of the free, phenomenological parameters and functions in the general model (7.3) in those various studies goes beyond the scope of our review. Our main focus in this section will be on the character of the mechanochemical coupling (cf. section 2.7) and the relevance of the thermal noise for the dynamics of the mechanical state variable in $(7.3)$, see also section 7.7 for a more systematic discussion of these points.

With more structural data of the actin-myosin system on the molecular level becoming available, A.F. Huxley and Simmons [611] already in 1971 came up with a more realistic modification of the original model, featuring a "fast" (chemical) variable with a small number of discrete states, tightly coupled to a "slow" (mechanical) continuous coordinate. For more recent studies along these lines see also 612 618 and references therein. A very recent, analytically solvable model, closely resembling A. F. Huxleys original setup and in quantitative agreement with a large body of experimental data, 
is due to 525.

The issue of the chemical to mechanical coupling has been for the first time addressed in detail by Mitsui and Oshima [144, pointing out that deviations from a simple and rigid one-to-one coupling may play an important role.

A connection between a model of the Huxley type with Feynman's ratchet-andpawl gadget has apparently been realized and worked out for the first time by Braxton and Yount [5, 6], though their model was later proven unrealistic by more detailed quantitative considerations 142, 143]. A similar Feynman-type approach has been independently elaborated by Vale and Oosawa [7]. More importantly, they seem to have been the first to bring into play the crucial question of the relative importance of the thermal fluctuations appearing in the dynamics of the mechanical coordinate (7.3) as compared to conformational (relaxational) changes powered by the chemical cycle (that is, ultimately by the power stroke).

One extreme possibility is characterized by barriers of the potentials $V_{m}(x)$ which can be crossed only with the help of the thermal noise $\xi(t)$ in (7.3), independently of how the chemical state $m$ evolves in the course of time. An example is the fluctuating potential ratchet (4.11) with $f(t)$ restricted to a discrete number $(M)$ of possible values, all smaller than unity in modulus. In such a case, the role of the chemical cycle is merely the breaking of the detailed balance, necessary for a manifestation of the ratchet effect in the $x$-dynamics. Moreover, the mechanochemical coupling is typically (i.e. unless the rates $k_{m \rightarrow m^{\prime}}(x)$ exhibit a very special, strong $x$-dependence, see below) loose, the number of chemical cycles per mechanical cycle randomly varies over a wide range.

The opposite possibility is represented by the traveling potential ratchet mechanism, see section 4.4. Each chemical transition $m \rightarrow m^{\prime}=m+1$ induces a strain in the mechanical coordinate via $V_{m^{\prime}}^{\prime}(x)$ in $(7.3)$ which then is released while $x$ relaxes towards the closest local minimum of $V_{m^{\prime}}^{\prime}(x)$. As $m$ proceed through the chemical loop, also the local minima of $V_{m}(x)$ are shifting forward in sufficiently small steps such that $x$ typically advances by one period $L$ after one chemical cycle. In this case, the thermal noise has only an indirect effect through the chemical rates $k_{m \rightarrow m^{\prime}}(x)$, but as far as the mechanical dynamics $(7.3)$ is concerned, almost nothing changes in comparison with a purely deterministic $(\xi(t) \equiv 0)$ behavior. In other words, the mechanochemical coupling is very rigid, the mechanical coordinate $x$ is almost exclusively powered by the chemical reaction and its behavior is basically "slaved" by the chemical transitions. The mechanical coordinate $x$ may at most play a role in that the practically deterministic relaxation of $x$ after a chemical transition $m \rightarrow m^{\prime}$ may delay the occurrence of the next transition $m^{\prime} \rightarrow m^{\prime \prime}$ until the new local minimum of $V_{m^{\prime}}(x)$ has been reached. Essentially, the system can thus be described by the chemical reaction cycle alone, possibly augmented by appropriate deterministic refractory periods (waiting times) after each reaction step [521, 522, 524]. It then does not seem any more appropriate to speak of a noise induced transport in the closer sense and the ratchet effect only enters the picture via the somewhat trivial traveling potential ratchet mechanism from section 4.4, see also section 2.7 .

A situation intermediate between these two extreme cases arises if the potential $V_{m}(x)$ exhibits (approximately) flat segments, requiring diffusion but no activated barrier crossing for being transversed. An example is the on-off ratchet scheme from section 4.2.

Another compromise between the two extremes consists in the following scenario: 
Thermally activated barrier crossing is unavoidable for the advancement of $x$. Yet, due to the choice of the rates $k_{m \rightarrow m^{\prime}}(x)$, the next chemical step becomes only possible after the respective barrier crossing has been accomplished. In other words, though thermal noise effects are an indispensable ingredient for the working of the motor enzyme model, the stepping of $x$ and $m$ is tightly coupled. Since the thermal activation processes can be considered as rate processes, such a model can be mapped in very good approximation to an augmented reaction cycle, with some mechanical states added to the chemical ones. The proper notion for such a situation seem to be "mechanochemical reaction cycle". For a more systematic treatment of such issues see section 7.7.

The conclusion of Vale and Oosawa [7] is that, within their Huxley-type model (7.3), thermal noise in the mechanical coordinate $x$ plays an important role; specifically, a mechanism similar to a fluctuating potential ratchet (section 4.3), a temperature ratchet model (sections 2.6 and 6.3), or a combination of both is postulated (later criticized as being unrealistic in [9, 142, 143]).

While the latter conclusion is mainly of a qualitative nature, a more quantitative investigation of the same question is due to Cordova, Ermentrout, and Oster [10], with the result that for cooperating motor enzymes like myosin, thermal activation processes are - within their choice of model parameters and functions in $(7.3)$ - crucial in the dynamics of the mechanical variable $x$, while for kinesin such processes may be of somewhat less importance. In deriving the latter conclusion, these authors go one step beyond Huxley's framework in that they also analyze the motion of a single head (no backbone), and especially of two heads without invoking a mean field approximation for the motion of the backbone, which, in this context should then rather be viewed as a "hinge" connecting the two heads.

A further refined variation of Huxley's model has been worked out by Leibler and Huse [8,9], together with a few-head model (beyond mean field) in a general spirit similar to that of Cordova, Ermentrout, and Oster [10]. In this model, however, a tight mechanochemical coupling is built-in from the beginning, namely the choice of the parameters and functions in $(7.3)$ is such that thermal noise effects on the mechanical coordinate $x$ play a minor role by construction. Furthermore, all the transition rates $k_{m \rightarrow m^{\prime}}$ are assumed to be independent of $x$. Within such a model, it is shown that at least $M=4$ chemical states are required to avoid incompatibilities with known experimental findings. The main achievement of these studies [8,9] is a unified description of "porter" motor proteins, e.g. kinesin, operating individually and spending a relatively short time detached from the polymer filament (moderate-to-large duty ratio), and of "rowers", e.g. myosin, which operate collectively and are characterized by a small duty ratio. Thus, "porters" are essentially processive, and "rowers" non-processive motor enzymes. A refined model similar in spirit has been put forward in [520].

\subsubsection{Jülicher-Prost model}

One of the most striking statistical mechanical features of interacting many body systems, both at and far away from thermal equilibrium, is the possibility of spontaneous ergodicity-breaking, entailing phase transitions, the coexistence of different (meta-) stable phases, and a hysteretic behavior in response to the variation of appropriate parameters. There is no reason why such genuine collective effects should not be expected also in Huxley-type mean field models, but it was not before 1995 that Jülicher and Prost [557] explicitly demonstrated the occurrence of those phenomena in such 
a model, see also [14, 601, 619 621. Specifically, they focused on the dependence of the average velocity $\langle\dot{x}\rangle$ upon (parametric) variations of the external force $F$ in (7.3), henceforth called $\langle\dot{x}\rangle$-versus- $F$ characteristics. As already mentioned, formally the crucial point in such a mean field approach is the appearance of a self-consistency equation for the "order parameter" $\langle\dot{x}\rangle$. Typically, this equation is nonlinear ${ }^{\mathbb{P}}$ and the existence of multiple (stable) solutions signals the breaking of ergodicity.

After having observed such a situation in their model, Jülicher and Prost pointed out in a subsequent work 622] the following remarkable consequence of the hysteretic $\langle\dot{x}\rangle$-versus- $F$ characteristics: If the rigid backbone is coupled to a spring, then an effective external force $F$ depending on the position of the backbone arises. If the spring is sufficiently soft, then the changes of $F$ are sufficiently slow such that the parametric $\langle\dot{x}\rangle$-versus- $F$ characteristics can be used. If this relation furthermore exhibits a hysteresis loop with the two $\langle\dot{x}\rangle$-versus- $F$ branches confined to either side of $\langle\dot{x}\rangle=0$, then a permanent periodic back-and-forth motion of the backbone is the result. Remarkably, strong indications for both, spontaneous ergodicity breaking (dynamical phase transition in the velocity-froce-relationship) as well as spontaneous oscillations can indeed be observed in motility assays 623 and in muscle cells under suitable conditions [14,601,619 622, 624 626], respectively.

We recall that spontaneous breaking of ergodicity with its above mentioned consequences is a common phenomenon already at equilibrium. In contrast, a finite current $\langle\dot{x}\rangle \neq 0$ at $F=0$ as well as spontaneous oscillations [627 631] represent genuine collective non-equilibrium effects which are excluded at thermal equilibrium by the second law of thermodynamics.

Both, from the conceptual viewpoint and with regard to the mechanochemical coupling issue, the Jülicher-Prost model exhibits a couple of noteworthy features. A first crucial assumption of the model is that not only the backbone itself but also the positions of the $N$ individual motors with respect to the backbone are perfectly rigid. Since the backbone moves with a speed $\langle\dot{x}\rangle$ it follows that for any single motor

$$
\dot{x}=\langle\dot{x}\rangle \text {. }
$$

Much like any intensive state variable in equilibrium thermodynamics, the "order parameter" $\langle\dot{x}\rangle$ within such a mean field approach is a macroscopic state variable and is not any more subject to any kind of random fluctuations in the thermodynamic limit $N \rightarrow \infty$. In other words, the stochastic equation for the single (uncoupled) motors (7.3) simplifies to an equivalent deterministic (noise-free) dynamics (7.26) for every single motor in the presence of a mean field (perfectly rigid all-to-all) coupling. Here $\langle\dot{x}\rangle$ plays the role of a formal (not yet explicitly known) deterministic force and - as already pointed out in section 7.4.1 - our next goal must now be to derive a self consistency equation for this order parameter $\langle\dot{x}\rangle$ if we wish to determine its explicit value. To this end we first notice that working with $(7.26)$ instead of $(7.3)$ is tantamount to setting $T=0$ and $-V_{m}^{\prime}(x)+F=\eta\langle\dot{x}\rangle$ in (7.3). Accordingly, the first two terms on the right hand side of the master equation $(7.8)$ may be replaced by the equivalent simplified expression $-\langle\dot{x}\rangle \partial P_{m}(x, t) / \partial x$.

A second essential assumption is that the $N$ individual motor enzymes are rooted in the backbone either at random positions or - biologically more realistic - with a constant spacing which is incommensurate with the period $L$ of the polymer filament.

\footnotetext{
${ }^{19}$ For an example, see equations $(\sqrt{7.28}), \sqrt{7.29}$ below.
} 
As a consequence, the reduced spatial distribution of particles $\sum_{m} \hat{P}_{m}(x, t)$ approaches an $x$ - and $t$-independent constant value for $N \rightarrow \infty$.

As a final assumption, a one-head description of the individual motor enzymes with $M=2$ chemical states is adopted 20 . Exploiting the above mentioned fact that $\hat{P}_{1}(x, t)+\hat{P}_{2}(x, t)$ is a constant and normalized on $[0, L]$ according to $(7.22)$, one can eliminate $\hat{P}_{2}(x, t)$ from the master equation $(\overline{7.8})$, yielding in the steady state (superscript $s t$ ) the ordinary first order equation [557]

$$
\langle\dot{x}\rangle \frac{d}{d x} \hat{P}_{1}^{s t}(x)=-\hat{P}_{1}^{s t}(x) k_{1 \rightarrow 2}(x)+\left[1 / L-\hat{P}_{1}^{s t}(x)\right] k_{2 \rightarrow 1}(x),
$$

supplemented by the periodic boundary condition ${ }^{22} \hat{P}_{1}^{s t}(x+L)=\hat{P}_{1}^{s t}(x)$. The unique solution is

$$
\hat{P}_{1}^{s t}(x)=\frac{\int_{x}^{x+L} d y k_{2 \rightarrow 1}(y) \exp \left\{\int_{x}^{y} d z \frac{k_{1 \rightarrow 2}(z)+k_{2 \rightarrow 1}(z)}{\langle\dot{x}\rangle}\right\}}{L\langle\dot{x}\rangle\left[\exp \left\{\int_{0}^{L} d z \frac{k_{1 \rightarrow 2}(z)+k_{2 \rightarrow 1}(z)}{\langle\dot{x}\rangle}\right\}-1\right]} .
$$

Note that the non-negativity of $\hat{P}_{1}^{s t}(x)$ is guaranteed if $k_{1 \rightarrow 2}(x) \geq 0$ and $k_{2 \rightarrow 1}(x) \geq 0$ for all $x$. Finally, by eliminating in the same way $\hat{P}_{2}(x, t)$ in the self-consistency equation (7.23) for $\langle\dot{x}\rangle$ one finds that

$$
\langle\dot{x}\rangle=\frac{1}{\eta}\left[F-\int_{0}^{L} d x\left(V_{1}^{\prime}(x)-V_{2}^{\prime}(x)\right) \hat{P}_{1}^{s t}(x)\right] .
$$

By introducing (7.28) into (7.29) a closed (transcendental) self consistency equation for the order parameter $\langle\dot{x}\rangle$ is obtained. Much like in the elementary mean field theory (Weiss theory) for a ferromagnet, the occurrence of multiple solutions will signal the breaking of ergodicity and thus a phase transition. Apart from the need of solving a transcendental equation at the very end, the above model is one of the very rare special cases (cf. section 4.3.1) of an analytically exactly tractable fluctuating potential ratchet. We finally recall that by interpreting the $M=2$ state model as a reduced $M=4$ state description, both rates $k_{1 \rightarrow 2}(x)$ and $k_{2 \rightarrow 1}(x)$ are still at our disposition (see section 7.4.2).

Besides the tremendous technical simplification of the problem, the most remarkable feature of the Jülicher-Prost model (7.28), (7.29) is that only the difference $V_{1}(x)-V_{2}(x)$ of the two potentials counts (one may thus choose one of them identically zero without loss of generality). It follows that the emerging qualitative results for a generic ( $L$-periodic and asymmetric) choice of $V_{1}(x)-V_{2}(x)$ will be valid independently of whether the mechanochemical coupling is loose (e.g. a dichotomously fluctuating potential ratchet with $V_{2}(x) \propto V_{1}(x)$, see section 4.3) or tight (e.g. a traveling twostate ratchet with $V_{2}(x)=V_{1}(x+L / 2)$, see section 4.4.2). Whether this feature should

\footnotetext{
${ }^{20}$ According to section 7.4.1, this model may equally well be viewed as a $M=2$ state model of enzymes with two highly coordinated heads. See also section 7.4 .2 for references and more details regarding such a model.

${ }^{21}$ The convergence towards a steady state in the long time limit is tacitly taken for granted. A partial justification of this ansatz can be given a posteriori by showing that such a solution indeed exists and moreover satisfies certain stability conditions against perturbations. Especially, the task to prove that no additional (non-stationary) long time solutions co-exist is a delicate issue. In practice, the only viable way consists in a direct numerical simulation of a large number $N$ of coupled stochastic equations.

${ }^{22}$ There is no normalization condition for $\hat{P}_{1}^{s t}(x)$ alone.
} 
be considered as a virtue (robustness) or shortcoming (oversimplification) of the model is not clear.

Modified Huxley-Jülicher-Prost type models have been explored by Vilfan, Frey, and Schwabl [632, 633]. Their basis is a $M=2$ state description of the single motors with a built-in tight mechanochemical coupling through the choice of the rates and potentials, but, at variance with Jülicher and Prost, without a completely rigid shape of the motors with respect to the backbone: unlike in (7.26), the center of mass of an individual motor may differ from the position where it is rooted in the backbone, say $\langle\dot{x}\rangle t$. Similarly as in Huxley's original work, the possibility of "strain" dependent (i.e. $x-\langle\dot{x}\rangle t$ dependent) rates $k_{m \rightarrow m^{\prime}}(x)$ plays an important role. With a rigid backbone, a mean field approach is still exact for $N \rightarrow \infty$ but technically more involved than in the Jülicher-Prost model, while resulting in qualitative similar collective phenomena [633]. In contrast, by admitting an elastic instead of a rigid backbone? the interaction between the motors is no longer of infinite range and corrections to a mean field approximation may become relevant under certain experimental conditions 632].

Another refined version of the Huxley-Jülicher-Prost setup, taking into account an extended number of biological findings, is due to Derenyi and Vicsek 635. While $M=4$ chemical states are included, only two different potential shapes $V_{m}(x)$ are proposed, one of them being identically zero, and a tight mechanochemical coupling is built in through the choice of the rates $k_{m \rightarrow m^{\prime}}(x)$. While a very good agreement with different experimentally measured curves is obtained, the issue of genuine collective phenomena is not specifically addressed.

Further studies of collective effects in coupled Brownian motors will be discussed in chapter 9 .

\subsection{Coordinated two-head model}

In this subsection we return to the description of a single motor enzyme with two heads within the general modeling framework (7.1)-(7.17). Especially, we recall that this model respects an invariance under a spatial displacement by one period if simultaneously the chemical states of the two heads are exchanged, see (7.5), (7.6). We furthermore recall that for a processive motor enzyme, i.e., one which can operate individually (for instance kinesin), the two heads need to coordinate their actions in order that at least one of them is always attached to the polymer filament.

Our goal is to approximately boil down the two-dimensional chemical state vector $m=\left(m_{1}, m_{2}\right)$ into an effective one-dimensional (scalar) description. To this end, we make the assumption that the two heads are so strongly coordinated that between subsequent steps there exists a time instant at which not only both heads are attached to the filament, but on top of that, the heads are in the same chemical state, $m_{1}=m_{2}$. Taking such a configuration as reference state, one of the two heads will be the first to make a chemical transition into another state. This may be, with certain, generically unequal probabilities, either the front or the rear head, and the chemical state may, again with typically unequal probabilities (cf. (7.16), (7.17)), either go one step forward or backward in its reaction cycle as time goes on ${ }^{2}$. Our central assumption is now,

\footnotetext{
${ }^{23} \mathrm{~A}$ computer animation (Java applet) which graphically visualizes the effect is available on the internet under 634.

${ }^{24}$ There seems to be no general agreement upon whether such inverse processes are possible with finite (however small) probability [18] or not [143].
} 
that once one of the heads has left the reference state $m_{1}=m_{2}$, the other head will not change its chemical state until the first one has returned into the reference state.

We are not aware of experimental observations which indicate that such a property is strictly fulfilled, but it appears to be an acceptable approximation, especially in view of the great simplification of the model it entails. Moreover, if one starts with a reduced description of the chemical cycle in each head in terms of only two effective states, based on a similar line of reasoning as in the preceding section 7.4.2, then necessarily one of these two states must correspond to the head being attached to the polymer filament and the other to the detached situation. Since both heads cannot be detached simultaneously, our assumption is thus automatically fulfilled in such a two-state description for each head.

If one makes the additional simplifying assumption that, starting from the reference state with both heads attached, only the rear head is allowed to detach, then an effective one-dimensional description of the chemical states of the two heads is straightforward: After the rear head has returned into the reference state, it either will have attached at the same binding site ( $\beta$-tubulin) from which it started out or it will have advanced to the next free binding site at a distance $2 L$. In the former case, it is again the same head which will make the next chemical reaction out of the reference state, while the other head continues to be stuck. In the latter case, the rear head has completed a stept5, $x \mapsto x+L$, and is now the new front head. If we additionally exchange the chemical labels $m_{1}$ and $m_{2}$ of the two heads then we are back in the original situation due to the symmetry of the system (7.5), (7.6). In other words, we have obtained an effective description in which only one of the chemical state variables, say $m_{1}$, can change, while $m_{2}$ is stuck all the time. Dropping the index of $m_{1}$, one readily sees that the effective potentials $V_{m}(x)$ and rates $k_{m \rightarrow m^{\prime}}(x)$ in this new description are now indeed $L$-periodic in $x$ and satisfy (7.25).

The more general case that out of the reference state $m_{1}=m_{2}$ both, the front and the rear head may detach from the filament with certain probabilities, can only be captured approximately by means of an effective one-dimensional chemical state variable ${ }^{26}$ : Namely, one has to assume that if the rear head detaches, then $x$ can only take values larger than the initial reference position $x_{\text {ref }}$ (but smaller than $x_{\text {ref }}+L$ ). Likewise, if the front head detaches, $x$ is restricted to $\left[x_{\text {ref }}-L, x_{\text {ref }}\right]$. These two possibilities can be imitated by "splitting probabilities" with which $x(t)$ in $(7.3)$ will evolve into the positive or negative direction after detachment by way of an appropriate choice of the potentials $V_{m}(x)$. Especially, these potentials have to be chosen such that a recrossing of $x_{\text {ref }}$ after detachment is practically impossible 27 . For the rest, the mapping to an effective one-dimensional chemical state variable $m$ with $L$-periodic potentials $V_{m}(x)$ and rates $k_{m \rightarrow m^{\prime}}(x)$ satisfying $(7.25)$ can be accomplished exactly like before.

\footnotetext{
${ }^{25}$ We recall that the stepping head itself advances by $2 L$, but the center of mass $x$ of the two heads only by $L$.

${ }^{26}$ The problem is that now the information about which of the two heads is chemically active (detached) must be uniquely encapsulated in $x$ in addition to the position of the center of mass.

${ }^{27}$ To be specific, we may model the chemical reference vector-state $m_{\text {ref }}$ with both heads attached by a potential $V_{m_{\text {ref }}}(x)$ with a very deep and narrow minimum at $x_{\text {ref }}$. If $m_{\text {ref }}$ goes over into one of the "neighboring" states, say $m^{\prime}$, then $V_{m^{\prime}}(x)$ should have pronounced maxima on either side of $x_{\text {ref }}$ such that $x(t)$ will proceed rather quickly and irrevocably away from $x_{\text {ref }}$, either to the right or left. The actual direction into which $x(t)$ disappears decides a posteriori whether it was the front or the rear head which has detached.
} 
Two noteworthy features which can be described within such a general modeling framework are thus (i) the possibility not only of "forward" but also of "backward" steps and (ii) the possibility that a head re-attaches to the same binding site from where it started out. Both these possibilities may be realized only with a small probability ${ }^{8}$ under "normal" conditions [587, 608, 636 but could become increasingly important [18, 510, 587, 637, 638] as the load force $F$ in $(7.3)$ approaches the "stopping force" or "stall load", characterized by zero net motion $\langle\dot{x}\rangle=0$ (cf. section 2.6.2).

\subsection{Further models for a single motor enzyme}

The above interpretation of (7.8), (7.18)-(7.25) as a model for a single motor enzyme with two highly cooperative heads has, to our knowledge, not been pointed out and derived in detail before?. However, practically the same model dynamics (7.3) has been used to describe the somewhat artificial scenario ${ }^{\beta 0}$ of a single head moving along a polymer filament [9, 10]. By changing the interpretation, such results can immediately be translated into our two-head setting.

As mentioned in section 7.4.3, models with two completely independently operating heads, except that they are connected by a "hinge", have been briefly addressed numerically in [10]. A refined model of this type has later been put forward and analyzed by Vilfan, Frey, and Schwabl [633] exhibiting good agreement with a variety of experimental curves and structural results.

Valuable contribution to the general conceptual framework [11] of single motor modeling and especially of the mechanochemical coupling [12] are due to Magnasco, see also 186. At variance with our present setup, the chemical processes within the entire motor enzyme are described from the beginning by a single, continuous chemical state variable [12] (see also sections 4.6 and 7.3.2). Published practically at the same time, models similar in spirit, but with only two discrete chemical states have been proposed by Astumian and Bier [15], by Prost, Chauwin, Peliti, and Ajdari [13], and by Peskin, Ermentrout, and Oster [17. The underlying picture is that, essentially, the motor enzyme as a whole is either "attached" to or "detached" from the protein filament. The emphasis in all these works [11, 13, 15, 17] (see also [644]) is put on the fundamental aspects and generic properties of motion generation in such systems; apart from the general features of spatial periodicity and broken symmetry, no contact with any further biological "details" is established. Yet, by using reasonable parameter values in a fluctuating potential sawtooth ratchet model, measured data for the average speed $\langle\dot{x}\rangle$ and the rate of ATP-consumption (cf. (7.23) and (7.24)) could be reproduced within an order of magnitude [15, 16]. On the other hand, it was demonstrated in [521,522] that even within the simplest two-state models for a single motor $(M=2$ in (7.18)), a large variety of even qualitatively contrasting results can be produced upon varying the model parameters. Not only a realistic choice of the model parameters but also of the details of the model itself is therefore indispensable.

\footnotetext{
${ }^{28}$ We remark that there are also models which rule out such backward steps a priori 143.

${ }^{29}$ Somewhat similar ideas can be found in [18, 551, 559, 601.

${ }^{30}$ Whether or not manipulated, single-headed kinesin can travel over appreciable distances on a microtubulus seems to be still controversial 18, 143, 569, 638 642. Remarkably, the experimental data from 642 could be fitted very well by an on-off ratchet model (A video illustrating motility data can be viewed on the internet under 643]). There seems to be evidence [577 that single-headed motion is fundamentally different from two-headed motion.
} 
A biologically well founded description of a motor enzyme with two cooperating heads, similar to our present setup with $M=2$ chemical states, has been introduced by Peskin and Oster 18]. A central point in this study is once more the relative roles of the thermal fluctuations and the relaxational processes due to release of chemically generated strain in the dynamics of the spatial coordinate $x$ in (7.3). Another important feature of the model is that, besides regular forward steps of the heads, also backward steps after detachment of the front head are admitted with a certain probability. The result, after fitting the model parameters to the experiment, is that within this specific model - thermal fluctuations play a minor role. Furthermore, it is found that backward steps are about 20 times less probable than forward steps 31 .

The model by Derenyi and Vicsek [510] is to some extent similar in spirit to the one by Peskin and Oster. Especially, backward steps are admitted and the two heads act highly cooperatively. The built-in mechanochemical coupling is a compromise in that thermal activation is indispensable but the rates $k_{m \rightarrow m^{\prime}}(x)$ are tailored such that the next chemical step can only occur after $x$ has crossed the respective barrier and is basically undergoing a purely mechanical relaxation. The model can be mapped almost exactly to a $M=2$ state model from section 7.2 .3 , though the original formulation 510 in terms of two rigid heads, coupled by a hinge and an "active" spring with variable rest length is admittedly more natural in this specific instance. In either case, the model can be described in very good approximation by an augmented reaction cycle with mechanical states properly added to the chemical states. The distinguishing feature of the model, the experimental justification of which remains unclear [568, 569, 645, 646] is that the two heads cannot pass each other: The distance between the front and the rear head (in other words, of the spring) can change but never become zero so that the heads never exchange their roles. The virtue of the model is its ability to fit very well various measured curves. The limiting case of a very strong "active" spring, such that thermal activation is no longer important, has been explored in 647]. A somewhat related model with two effectively asymmetric heads is due to [451], see also section 6.5 .

The conceptual framework [15] of Astumian and Bier for modeling single molecular motors has been further developed and refined in a remarkable series of works [16, 54, 55, 187, 352, 560, 648, 649. Various aspects and results of their central study [16] have been repeatedly referred to already in the outline of our general modeling framework. The chief points in [16] are a comprehensive discussion of the mechanochemical coupling problem and the conclusion that many experimental indications and theoretical arguments seem to be compatible with a rather loose coupling, especially when a suitably augmented cooperative two-head model is invoked [648, 649]. A complementary discussion along a closely related spirit is given in 644]. Especially worth mentioning is that the fluctuational analysis of measured single motor protein trajectories in [609] is incompatible with a certain class of very simple (fluctuating potential) ratchet models but have been demonstrated in 648, 649] to be perfectly reproducible by means of a more elaborated and refined description.

Non-cooperative discrete-state models with a built-in tight mechanochemical coupling in the spirit of [8, 9, 164, 520] (see at the end of section 7.4.3) have been addressed in [186, 521 526], especially with respect to their behavior under the influence of an external load $F$. Notwithstanding the conclusion in [8,9] that at least four states are

\footnotetext{
${ }^{31}$ See also the discussion at the end of the previous subsection.
} 
necessary for a realistic model, the agreement of the two- and three-state models proposed in [521, 522, 526] and [525], respectively, with experimental observations is quite good. Various generalizations of these "mechanochemical reaction cycle models" (cf. sections 7.4.3 and 7.7) are due to [523], while the extension to general waiting time distributions has been addressed in [521, 522, 524], admitting in addition to thermally activated mechanochemical rate processes e.g. also the description of mechanical relaxation of strain. Their drawback is a large number of additional phenomenological model parameters.

The viewpoint [13] of Prost and collaborators with respect to modeling single motor enzymes has been further elaborated in [14,619] and especially in [551, 599 601]. While the general framework has much in common with that of Astumian and Bier, these workers put special emphasize on the possible relevance of "active sites", i.e. a pronounced dependence of the transition rates $k_{m \rightarrow m^{\prime}}(x)$ on the mechanical state $x$, such that transitions are practically excluded outside of certain small $x$-regions. They furthermore leave room to the possibility that a traveling potential ratchet mechanism may dominate over a possibly coexisting fluctuating potential ratchet mechanism, in which case the mechanochemical coupling might be rather tight. An explicit modeling of cooperative two-headed motor enzymes along somewhat similar lines as in section 7.5 is briefly mentioned in 551, 601. The resulting description with $M=2$ effective chemical states associates each state with one of the heads being bound to the filament and the other detached. One thus recovers the traveling potential ratchet model from [40], advancing in discrete steps of $L / 2$ as detailed in section 4.4.2. The influence of an external load $F$ on velocity and processivity (detachment rate of the molecular motor from the microtubulus) has been addressed in [599], see also [523]. A related study due to [600] suggests a loose mechanochemical coupling at least under heavy load. The case that the load is not an externally imposed constant force but rather is due to the "cargo", modeled as additonal relevant dynamical variable that interacts with the motor via an elastic coupling, has been addressed in [511, 512], see also below equation (7.4).

A detailed analysis of a somewhat extended model class with pronounced "active sites" and a strong traveling potential component has been carried out in [559, 598,650]. In agreement with experiments, these models reproduce a "saturation" of the current $\langle\dot{x}\rangle$ as a function of the ATP-concentration 13, 14, 619, captured by a Michaelis-Menten relation for a large class of moderately and strongly cooperative models under zero lead $F$ [559, 598, 650], while for finite load a somewhat modified quantitative behavior is expected [559, 598, 600, 646]. We remark that while for cooperative two-head models with only $M=2$ chemical (or "internal") states per head, the assumption of "active sites" is indeed indispensable for such a saturation of the current, the same is no longer true as soon as $M>2$.

\subsection{Summary and discussion}

We close with some general remarks regarding the modeling of molecular motors as reviewed in this chapter. Previously introduced notions and facts are freely used without explaining them or citing the original literature again. To some extent, this discussion continues and makes more precise those from sections 4.6 and 7.4.3.

The general importance of asymmetry induced rectification, thermal fluctuations, and the coupling of non-equilibrium enzymatic reactions to mechanical currents ac- 
cording to Curie's principle for intracellular transport processes is long known [23, 24]. The present framework has the virtue that it is based on a quantitative microscopic modeling and as such is not restricted to the linear response regime close to thermal equilibrium. Within this general framework, roughly speaking two approaches of modeling molecular motors may be distinguished: The first, "traditional" one is a bottom-up-type strategy, starting with a certain set of biological facts (measurements and more or less "basic" conclusions therefrom) and then constructing an "ad hoc" model on this basis. The second is the top-down-type approach, followed to some extent in more recent works based on the "ratchet paradigm" and elaborated in full detail in our present chapter.

Our first main conclusion is that all models known to this author are compatible (possibly after some mapping or transformation of state variables) with the basic framework from section 7.2 , and most of them also with the simplified description in terms of a single mechanical state variable $x$ and the corresponding model dynamics (7.3), identified below (7.14) as a (generalized) pulsating ratchet scheme. In other words, such an approach is not in contradiction with "traditional" biological models, but may well offer a fresh and more systematic (top-down) view of things [12, 186, 576, 648, 649, 651].

Within this still very general class of models (7.3) the most realistic choice of model parameters and model functions is still under debate and certainly also depends on the specific type of molecular motor under consideration (especially whether it is of processive (individually acting) or non-processive (collectively acting) nature or even consists of a single motor domain (head) only). Conversely, it is remarkable that all these different species can be treated within one general framework.

Three basic questions in this respect, which are not always sufficiently clearly separated from each other, regard: (i) The possibility of an (approximate) description in terms of a single (effective) chemical state variable. (ii) The relative importance of the thermal fluctuations appearing in the dynamics of the mechanical coordinate (7.3) as compared to conformational changes powered by the chemical cycle. (iii) The character (loose or tight) of the mechanochemical coupling.

The answer to these questions may not only depend on the type of molecular motor under consideration (see above) but also on whether an external load $F$ is acting and possibly on still other external conditions. For example, it may well be that, as the load $F$ increases, the relative importance in (7.3) of thermal activated barrier crossing and deterministic relaxation processes (i.e. the answer to question (ii) above) considerably changes. The reason is that, as the force $F$ increases, existing effective potential barriers in the stochastic dynamics (7.3) may disappear and new ones appear ${ }^{32}$. Similarly, the external load $F$ may also significantly change the character of the mechanochemical coupling 53 [16, 510, 525, 559, 587, 598 600, 637, 652, 653, (i.e. the answer to question (iii) above).

The answer to the first of the above questions depends on the cooperativity of the two heads: If they act completely independently of each other, they can obviously be described individually, and a single (scalar) chemical state variable $m$ for each head is then sufficient. That the same may be possible for a very strong coordination of the heads has been demonstrated in section 7.5. On the other hand, if the cooperativity is loose but non-negligible, then a reduction of the two-dimensional chemical state space (cf. (7.1)) is impossible.

\footnotetext{
${ }^{32}$ The "total" or "effective" potential in (7.3) is given by $V_{m}(x)-x F$.

${ }^{33}$ Especially, $F$ may change the shape of the potentials $V_{m}(x)$, as discussed below equation (7.4).
} 
The second question is sometimes also discussed under the label of power-stroke versus motor-diffusion modeling strategy [143, 525, 653. In the first case, the chemical cycle "slaves" the mechanical cycle by creating a sequence of strong mechanical strains (power strokes) that are released by concomitant, basically deterministic changes of the mechanical state (geometrical shape). Typically, there is little back reaction of the mechanical to the chemical coordinate, and we are thus essentially dealing with a genuine traveling potential ratchet scheme. In the second case, thermal fluctuations play a major role in the dynamics of the mechanical state variable $(7.3)$. The first model of this type goes once again back to Huxley [4] and the apparent lack of strong experimental support for the power stroke concept [653] has served as a motivation for various other such models ever since. Especially, this controversy has a long history already within the realm of "traditional" biological modeling and the gain of new insight in this repect from an approach based on the "ratchet paradigm" may be limited. Also, we may emphasize once more that in either case thermal noise plays a crucial role with respect to the chemical reaction cycle - in this sense any model of a molecular motor (not only those of the motor-diffusion type) "rectifies" thermal fluctuations. We further remark that also within a motor-diffusion modeling, the mechanochemical coupling may still be either tight (e.g. Huxleys model) or loose (e.g. the on-off ratchet). On the other hand, a power-stroke model always implies a tight mechanochemical coupling.

Another related question within a motor-diffusion modeling is whether the thermal fluctuations acting on the mechanical state variable can be treated within the activated barrier crossing limit (see section 3.8) or whether free diffusion-like behavior plays a significant role. Only in the former case, a description of the mechanical state variable in terms of discrete states and transition rates between them is admissible, see section 6.7 and [521, 522, 524, 526]. Note that both options are still compatible with either a tight or a loose mechanochemical coupling. In the case of a tight coupling in combination with an activated barrier crossing description, a so-called "mechanochemical reaction cycle" arises (cf. section 7.4.3). Since from a fundamental viewpoint, the distinction between chemical and mechanical state variables is somewhat arbitrary anyway (see section 7.2.2), we are then basically recovering an effective power-stroke model.

We finally come to the question (iii) of the mechanochemical coupling (see also section 2.7). We first remark that a tight coupling not necessarily means that the chemical state variable always "slaves" the mechanical one (genuine power-stroke model) but that one variable "slaves" the other at each stage of the mechanochemical reaction cycle (the chemical reaction may be blocked - due to active sites, i.e. stronly $x$-dependent rates $k_{m \rightarrow m^{\prime}}(x)$ - until some mechanical transition between different geometrical shapes of the motor has been accomplished, e.g. in the above effective power-stoke model). Restricting ourselves to the simplest case of a one-dimensional chemical state variable, a tight mechanochemical coupling means that a description in terms of a single effective state variable is possible, and a loose coupling means that such a description is impossible. In other words, the state space is either essentially one- or two-dimensional. In the first case, there is a unique "pathway" in the $x$ - $m$-space, in the second case bifurcations exist. Examples are genuine traveling potential ratchets and fluctuating potential ratchets, respectively. We, however, emphasize that the conclusion suggested by the latter example, namely that a loose mechanochemical coupling implies that thermal fluctuations play an essential role in the dynamics of the mechanical coordinate (7.3), can be easily demonstrated as incorrect by counterexamples. In other words, the ther- 
mally induced randomness of the chemical reactions suffices to produce bifurcations in the "pathway" through the full $x$ - $m$-space.

The possibility of a loose mechanochemical coupling is widely considered as one of the main conceptually new aspect of the "ratchet paradigm" as compared to "traditional" biological models34. However, in its simplest and most pronounced form, namely the fluctuating potential ratchet scheme from section 4.3 (i.e. with $x$-independent rates $\left.k_{m \rightarrow m^{\prime}}\right)$ it is apparently incompatible with the fluctuational analysis of single (two-headed) motor protein trajectories 609. On the other hand, the experimental data for single-headed kinesin from [642 could be fitted very well to an on-off ratchet model. The currently prevailing opinion seems to be that a loose coupling is unlikely for processive motors like two-headed kinesin but a realistic option in the case of non-processive (cooperative or single-headed) motors [525, 568, 569,605, 606, 608, 609, 618, 636, 642, 646, 653 656]. However, room for the possibility of a loose coupling even in the case of kinesin is still left e.g. in [16, 587, 637, 657]. If one considers the concept of a loose mechanochemical coupling as the only substantial new contribution of the "ratchet paradigm" to the modeling of molecular motors, then - in the so far absence of striking experimental indications of such a coupling the merits of this paradigm may still be considered as questionable. However, such a viewpoint may not do due justice to other noteworthy achievements like the prediction of new collective effects from section 7.4.4 or the unified new view and working model.

\footnotetext{
${ }^{34}$ Sometimes, also the possibility of a motor-diffusion modeling approach is considered as such.
} 


\section{Chapter 8}

\section{Quantum ratchets}

For many of the so far discussed ratchet systems, especially those for which thermal fluctuations play any significant role, the characteristic length-, energy- etc. scales are very small and it is thus just one more natural step forward to also take into account quantum mechanical effects.

Before we enter the actual discussion of such effects, two remarks are in place: First, we have encountered in sections 5.3.3 and 5.6 theoretical models and experimental realizations of Josephson and SQUID ratchet systems. Since the basic state variables in such devices are phases of macroscopic quantum mechanical wave functions, it is tempting to classify them as quantum ratchet systems. Our present viewpoint, however, it that the decisive criterion should be the classical or quantum mechanical character of the effective dynamics governing the relevant state variables of a system, independently of whether the microscopic basis of this effective dynamics is of classical or quantum mechanical nature, see also [94]. For instance, the existence of stable atoms, molecules, and solids is clearly a genuine quantum mechanical phenomenon, yet a classical theory of gases, liquids, and solids can be established. From this viewpoint, the Josephson and SQUID ratchet systems as discussed in sections 5.3.3 and 5.6 are thus classical ratchets. The realization of a full-fledged quantum mechanical ratchet according to our present understanding in SQUID systems will be discussed later in section 8.4. As a second remark we mention that the proper quantum mechanical treatment, e.g. of the Seebeck effect (section 6.1) or the photovoltaic effects (section 5.2), may arguably be considered as very early quantum ratchet studies of considerable practical relevance. However, in the present section we put our main emphasis not on a faithful quantum mechanical modeling of such specific systems but rather on the exploration of the basic features of much simpler models. Namely, our main focus will be on the interplay between tunneling and the effects induced by the thermal environment (i.e. dissipation and thermal noise) in the quantum mechanical counterparts of the classical tilting ratchet dynamics (5.1).

\subsection{Model}

In the case of classical Brownian motion, we have introduced in section 2.1.2 a model which takes into account the influence of the thermal environment along a rather heuristic line of reasoning, see also sections 2.9 and 3.4.1. In contrast, on a quantum mechanical level, such a heuristic modeling of dissipation and thermal noise, e.g. on 
the level of the Schrödinger equation, is much more problematic and liable to subtle inconsistencies for instance with the second law of thermodynamics or some basic principles of quantum mechanics, see [100,243, 658 663] and further references therein. To avoid such problems, we follow here the common route [66, 84, 94 96, 189, 664 667] to describe both the system and its thermal environment within a common Hamiltonian framework, with the heat bath being modeled by an infinite set of harmonic oscillators. Especially, within a quantum mechanical approach, keeping a finite mass of the system is unavoidable, i.e. a quantum ratchet is by nature endowed with finite inertia. If one insists in considering the overdamped limit $m \rightarrow 0$ then this limit usually has to be postponed to the very end of the calculations.

Similarly as in section 3.4.1, our starting point is a one-dimensional quantum particle with mass $m$ in an asymmetric, periodic ratchet-potential $V(x)$ of period $L$ in the presence of a tilting force field $y(t)$ that is unbiased on average. This bare system is furthermore coupled via coupling strengths $c_{j}$ to a model heat bath of infinitely many harmonic oscillators with masses $m_{j}$ and frequencies $\omega_{j}\left(\omega_{j}>0\right.$ without loss of generality) yielding the compound (system-plus-environment) Hamiltonian

$$
\begin{aligned}
\mathbf{H}(t) & =\frac{\mathbf{p}^{2}}{2 m}+V(\mathbf{x})-\mathbf{x} y(t)+\mathbf{H}_{B} \\
\mathbf{H}_{B} & :=\sum_{j=1}^{\infty} \frac{\mathbf{p}_{j}^{2}}{2 m_{j}}+\frac{1}{2} m_{j} \omega_{j}^{2}\left(\mathbf{x}_{j}-\frac{c_{j} \mathbf{x}}{m_{j} \omega_{j}^{2}}\right)^{2} .
\end{aligned}
$$

Here, $\mathbf{x}$ and $\mathbf{p}$ are the one-dimensional coordinate and momentum operators of the quantum Brownian particle of interest, while $\mathbf{x}_{j}$ and $\mathbf{p}_{j}$ are those of the bath oscillators. As initial condition at time $t=0$ we assume that the bath is at thermal equilibrium and is decoupled from the system. The infinite number of oscillators guarantees an infinite heat capacity and thus a reasonable model of a heat bath that keeps its initial temperatures for all later times' $t>0$. For the rest, it turns out 66, 95, 96, 664 667 that the effect of the environment on the system is completely fixed by the frequencies $\omega_{j}$ and the ratios $c_{j}^{2} / m_{j}$, or equivalently, by the so called spectral density

$$
J(\omega):=\frac{\pi}{2} \sum_{j=1}^{\infty} \frac{c_{j}^{2}}{m_{j} \omega_{j}} \delta\left(\omega-\omega_{j}\right) .
$$

By way of integrating out the bath degrees of freedom in (8.1) one obtains 666,95, 96, 664 667 the following one-dimensional Heisenberg equation for the position operator $\mathbf{x}(t)$ :

$$
m \ddot{\mathbf{x}}(t)+V^{\prime}(\mathbf{x}(t))-y(t)=-\int_{0}^{t} \hat{\eta}\left(t-t^{\prime}\right) \dot{\mathbf{x}}\left(t^{\prime}\right) d t^{\prime}+\boldsymbol{\xi}(t) .
$$

Like in (2.1), the left hand side can be associated to the bare system dynamics, while the right hand side accounts for the influence of the environment through the damping kernel

$$
\hat{\eta}(t):=\frac{2}{\pi} \int_{0}^{\infty} d \omega \omega^{-1} J(\omega) \cos (\omega t) .
$$

\footnotetext{
${ }^{1}$ Further shortcomings of a heat bath with a finite number of oscillators are: (i) Both the memory kernel 8.5) and the noise-correlation (8.7) do not decay to zero for large times, rather they are quasiperiodic. (ii) The future behavior of the "noise" (8.6) becomes perdictable from its past, at least in the classical limit, see section 11-5 in [668].
} 
and the operator valued quantum noise

$$
\boldsymbol{\xi}(t)=\sum_{j=1}^{\infty} c_{j}\left(\frac{\mathbf{p}_{j}(0)}{m_{j} \omega_{j}} \sin \left(\omega_{j} t\right)+\left(\mathbf{x}_{j}(0)-\frac{c_{j} \mathbf{x}(0)}{m_{j} \omega_{j}^{2}}\right) \cos \left(\omega_{j} t\right)\right),
$$

containing the initial conditions of the bath and of the particle's position. Exploiting the assumed thermal distribution of the bath $\mathbf{H}_{B}$ at $t=0$ one finds [66, 95, 96, 664 667 that $\boldsymbol{\xi}(t)$ becomes a stationary Gaussian noise with mean value zero. Moreover, one recovers the usual connection (via $J(\omega)$ ) between the random and the friction effects of the bath on the right hand side of (8.4) in the form of the fluctuation-dissipation relation

$$
\langle\boldsymbol{\xi}(t+\tau) \boldsymbol{\xi}(t)\rangle=\frac{\hbar}{\pi} \int_{0}^{\infty} d \omega J(\omega)\left[\operatorname{coth}\left(\frac{\hbar \omega}{2 k_{B} T}\right) \cos (\omega \tau)-i \sin (\omega \tau)\right],
$$

where $\langle\cdot\rangle$ indicates the thermal average (quantum statistical mechanical expectation value), $i:=\sqrt{-1}$, and $\tau \geq 0$.

In the following, we will focus on a so called Ohmic bath, characterized by a linear initial growth of the spectral sensity $J(\omega)$, a "cutoff" frequency $\omega_{c}$, and a "coupling parameter" $\eta$ :

$$
J(\omega)=\eta \omega \exp \left\{-\omega / \omega_{c}\right\}
$$

The cutoff $\omega_{c}$ is introduced in order to avoid unphysical ultraviolet divergences but will always be chosen much larger than any other relevant characteristic frequency of the model. The special role of such an Ohmic heat bath becomes apparent by observing that the corresponding damping kernel (8.5) approaches

$$
\hat{\eta}(t)=2 \eta \delta(t)
$$

when the cutoff $\omega_{c}$ goes to infinity. The integral in (8.4) thus boils down to the memoryless viscous friction $-\eta \dot{\mathbf{x}}(t)$. In other words, $\eta$ in (8.8) has the meaning of a damping coefficient due to viscous friction.

In the classical limit, i.e., for $\hbar / k_{B} T$ much smaller than any other characteristic time scale of the noiseless system (8.4), the correlation (8.7) with (8.5) correctly approaches the classical fluctuation-dissipation relation from (6.23). Furthermore, in this limit all quantum fluctuations vanish, so that q-numbers go over into c-numbers and (8.4) reproduces (for $y(t) \equiv 0$ ) the classical model (6.22) of a real valued stochastic process $x(t)$ in the presence of Gaussian noise $\xi(t)$ and (2.1) in the special case of a memoryless damping (8.9), see also section 3.4.1.

For later purposes, it is useful to distinguish between two different variants of the classical limit: The first one, which we call formal classical limit, consists in letting $\hbar \rightarrow 0$, i.e. quantum effects are simply ignored within such a description, independent of how relevant they are in the true system under study. This limit is formal in so far as in reality $\hbar$ is a natural constant. A second possibility, which we call physical classical limit, consists in focusing on large temperatures $T$ such that $\hbar / k_{B} T$ is sufficiently small and thus quantum effects become indeed negligible in the real system.

As suggested by the above mentioned findings in the classical limit, the harmonic oscillator model for the thermal environment 8.2), (8.3), (8.8), provides a rather satisfactory description in a large variety of real situations [66, 94 96, 665, 666, 669, 670], 
even though for many complex systems, one does not have a very clear understanding of the actual microscopic origin of the damping and fluctuation effects. In fact, it seems to be widely believed that once the dissipation mechanism is known to be of the general form appearing on the right hand side of equation (8.4), i.e. to be a linear functional of the system velocity, then for a heat bath at thermal equilibrium all the statistical properties of the quantum noise $\boldsymbol{\xi}(t)$ in (8.4) are uniquely fixed, i.e. independent of any further microscopic details of the thermal heat bath. Arguments in favor of this conjecture have been given e.g. in [81, 94, 95, 189, 671], but a veritable proof does not seem to exist yet, see also sections 2.1.2, 3.4.1 and [80, 92, 93, 97] for the classical limit. Under the assumption that the conjecture holds, it can be inferred 95. that any dissipative dynamics of the form (8.4) which is in contact to an equilibrium heat bath can be represented by a harmonic oscillator model (8.1), (8.2). This does not mean that in every such physical system the actual bath is a harmonic oscillator bath, but only that one cannot tell the difference as far as the behavior of the system $\mathbf{x}(t)$ is concerned 95. We finally remark that the damping kernel (8.5) does not change in the classical limit, it is the same for both a quantum mechanical or classical treatment of the system dynamics. In other words, the knowledge of the dissipation term in the classical limit appears to be sufficient to completely fix the quantum mechanical stochastic dynamics.

Historically, the harmonic oscillator model has apparently been invoked for the first time by Einstein and Hopf [78 for the description of an oscillating electrical dipole under blackbody irradiation and subjected to radiation damping2 A classical model with a harmonic oscillator potential $V(x)$, but otherwise exactly like in (8.1)-8.3) has been put forward by Bogolyubov [675], however, without explicitly working out the statistical properties of the fluctuations $\xi(t)$, especially their Gaussian character and the classical counterpart $(\sqrt[6.22]{ })$ of the fluctuation-dissipation relation (8.5), (8.7). The latter issues, together with a quantum mechanical transcription of the model, has been accomplished by Magalinskii [84]. Subsequent re-inventions, refinements, and generalizations of the model have been worked out e.g. in [85, 88, 89, 94, 95, 189, 664.

\subsection{Adiabatically tilting quantum ratchet}

For general driving $y(t)$, equation (8.4) gives rise to a very complicated non-equilibrium quantum dynamics. To simplify matters [161, 676, 677], we restrict ourselves to very slowly varying tilting forces $y(t)$ such that the system can always adiabatically adjust to the instantaneous thermal equilibrium state (accompanying equilibrium). We furthermore assume that $y(t)$ is basically restricted to the values $\pm F$, i.e., the transitions between $\pm F$ occur on a time scale of negligible duration in comparison with the time the particle in (8.4) is exposed to either of the "tilted washboard" potentials

$$
U^{ \pm}(x):=V(x) \mp F x
$$

cf. figure 8.1. As a final assumption we require a positive but not too large $F$, such that $U^{ \pm}(x)$ still display a local maximum and minimum within each period $L$. Apart from this, the tilting force $y(t)$ may still be either of stochastic or of deterministic nature.

\footnotetext{
${ }^{2}$ A preliminary toy-model, somewhat related to the problem considered by Einstein and Hopf is due to Lamb 672]. It can be mapped onto a harmonic oscillator model 95 but does not involve fluctuations of any kind. The same proviso applies for further related early works, like e.g. [673, 674].
} 


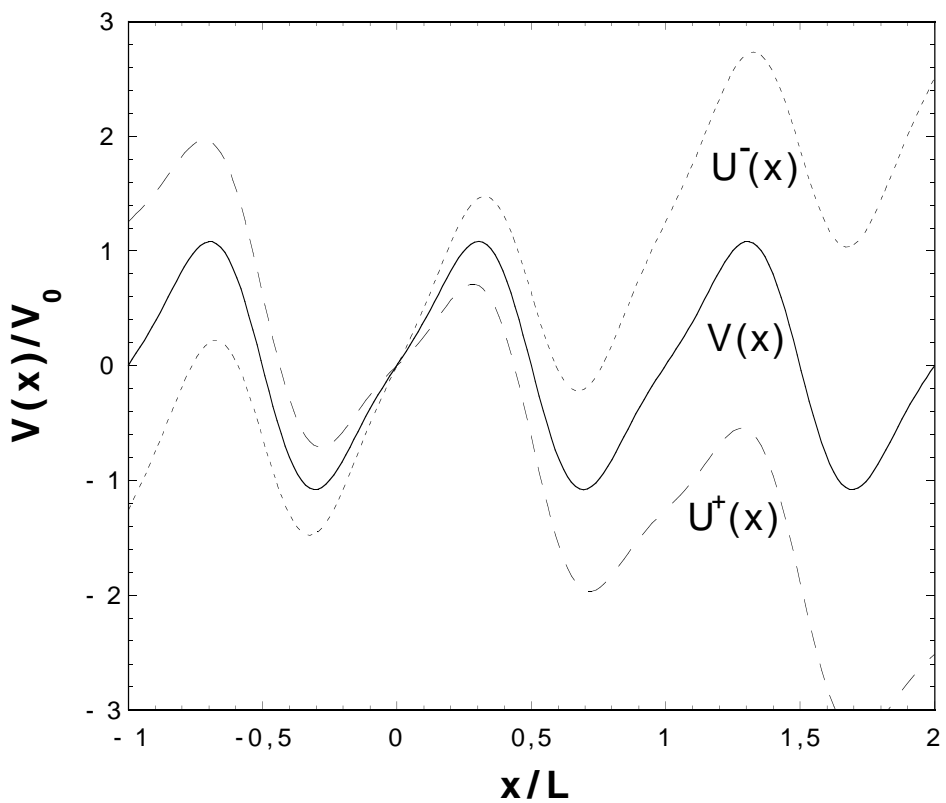

Figure 8.1: Solid: The ratchet potential $V(x)=V_{0}[\sin (2 \pi x / L)-0.22 \sin (4 \pi x / L)]$. Note that this potential is almost identical to the spatially inverted potential from (2.3), see also figure 2.2. Dashed and dotted: "tilted washboard potentials" $U^{ \pm}(x)$ in (8.10) with $F l=0.1 V_{0}, l=L / 2 \pi$.

Within the so defined model, we are essentially left with six model parameters 3 , namely the particle mass $m$, the "potential parameters" $V_{0}, L$, and $F$ (see figure 8.1), and the "thermal environment parameters" $\eta$ and $T$. We now make the assumption that these parameters are chosen such that a classical particle which starts at rest close to any local maximum of $U^{ \pm}(x)$ will deterministically slide down the corresponding slope but will not be able to subsequently surmount any further potential barrier and so is bound to end in the next local minimum. Differently speaking, a moderate-tostrong friction dynamics is considered and deterministically "running solutions" are excluded.

We further assume weak thermal noise, that is, any potential barrier appearing in (8.10) is much larger than the thermal energy, i.e.

$$
\Delta U^{\min } \gg k_{B} T
$$

where $\Delta U^{\text {min }}$ denotes the smallest of those potential barriers. As a consequence, we are dealing with an barrier crossing problem (see section 3.8) and thus the average particle current in either of the two potentials $U^{ \pm}(x)$ can be expressed in terms of two rates according to (3.55), see also (5.6). Moreover, the assumption of rare jumps of $y(t)$ between the two values $\pm F$ makes it possible to express the net current by way of an adiabatic limit argument analogous to (5.2), (5.9) in terms of these two partial

\footnotetext{
${ }^{3}$ Throughout this section the cutoff $\omega_{c}$ in 8.8 is chosen much larger than any other characteristic frequency of the system and therefore does not appear any more in the following.
} 
currents. In this way, one finally arrives at the following expression for the averaged net particle current in terms of two rates:

$$
\langle\dot{x}\rangle=\frac{L}{2}\left(1-e^{-F L / k_{B} T}\right)\left(k_{r}^{+}-k_{l}^{-}\right) .
$$

Here, $k_{r}^{+}$indicates the escape rate from one local minimum of $U^{+}(x)$ to its neighboring local minimum to the right, and similarly $k_{l}^{-}$denotes the rate to the left in the potential $U^{-}(x)$. We also recall that the average on the left hand side of (8.12) indicates a thermal averaging (quantum statistical mechanical expectation value) together with an averaging over the driving $y(t)$.

Within a purely classical treatment of the problem, i.e. within the formal classical limit $\hbar \rightarrow 0$, any of the two rates $k$ in (8.12) describe thermally activated transitions "over" a certain potential barrier $\Delta U$ between neighboring local minima of the corresponding potential. Due to the weak noise condition (8.11), such a rate $k$ is given in very good approximation by the well known Kramers-rate expression 66

$$
\begin{aligned}
& k=\frac{\mu \sqrt{U^{\prime \prime}\left(x_{0}\right)}}{2 \pi \sqrt{\left|U^{\prime \prime}\left(x_{b}\right)\right|}} e^{-\Delta U / k_{B} T} \\
& \mu:=\frac{\sqrt{\eta^{2}+4 m\left|U^{\prime \prime}\left(x_{b}\right)\right|}-\eta}{2 m},
\end{aligned}
$$

where $x_{b}$ and $x_{0}$ denote the above mentioned local potential-maximum and -minimum, respectively, and where indices $r, l$, and \pm have been dropped. Note that in the overdamped limit $m \rightarrow 0$, the Kramers-Smoluchowski rate-expression from (2.45) is recovered.

Turning to a quantum mechanical treatment of the problem, the rates in (8.12) in addition have to account for quantum tunneling "through" the potential barriers. Especially, due to our assumption that moderate-to-strong friction is acting, the tunneling dynamics is incoherent and a quantum rate description of the current (8.12) is valid. To evaluate these rates, a sophisticated line of reasoning has been elaborated 66, 96, 665]. Starting with the Hamiltonian system-plus-reservoir model (8.1) and adopting the so-called "imaginary free energy method" [66, 678] or, equivalently, the "multidimensional quantum transition state theory" [66, 679, 680, it is possible to express the escape rate $k$ in terms of functional path integrals. After integration over the bath modes and a steepest descent approximation, one obtains the semiclassical form

$$
k=A e^{-S / \hbar} .
$$

Here, the exponentially dominating contribution $S$ is defined via the nonlocal action

$$
S_{b}[q]:=\int_{0}^{\hbar / k_{B} T} d \tau\left[\frac{m \dot{q}^{2}(\tau)}{2}+U(q(\tau))+\frac{\eta}{4 \pi} \int_{-\infty}^{\infty} d \tau^{\prime}\left(\frac{q(\tau)-q\left(\tau^{\prime}\right)}{\tau-\tau^{\prime}}\right)^{2}\right] .
$$

This action has to be extremized for paths $q(\tau)$ under the constraints that $q(\tau+$ $\left.\hbar / k_{B} T\right)=q(\tau)$ for all $\tau$, and that there exists a $\tau$-value such that $q(\tau)=x_{b}$. A trivial such extremizing $q(\tau)$ is always $q(\tau) \equiv x_{b}$. Among this and the possibly existing further extrema one selects the one that minimizes $S_{b}[q]$, say $q_{b}(\tau)$, to obtain

$$
S:=S_{b}\left[q_{b}\right]-\hbar \beta U\left(x_{0}\right)
$$


The pre-exponential factor $A$ in (8.15) accounts for fluctuations about the semiclassically dominating path $q_{b}(\tau)$.

For a numerical exemplification [161, 676, 677] we use $T$ as control parameter and fix the five remaining model parameters $m, \eta, V_{0}, F$, and $l:=L / 2 \pi$. Without specifying a particular unit system this can be achieved by prescribing the following five dimensionless numbers: First we fix $V_{0}, F, l$ and thus $U^{ \pm}(x)$ through $F l / V_{0}=0.2$, $\Delta U^{\min } / V_{0}=1.423$, and $\left|U^{+\prime \prime}\left(x_{b}\right)\right| l^{2} / V_{0}=1.330$ corresponding to the situation depicted in figure 8.1. Next we choose $\eta / m \Omega_{0}=1$ with $\Omega_{0}:=\left[V_{0} / l^{2} m\right]^{1 / 2}$, meaning a moderate damping as compared to inertia effects. To see this we notice that $\Omega_{0}$ approximates rather well the true ground state frequency $\omega_{0}^{+}:=\left[U^{+\prime \prime}\left(x_{0}\right) / m\right]^{1 / 2}$ in the potential $U^{+}(x), \omega_{0}^{+}=1.153 \Omega_{0}$, and similarly for $U^{-}(x)$. In particular, $\eta / m \Omega_{0}=1$ strongly forbids deterministically running solutions. In order to specify our last dimensionless number we remark that within the weak noise assumption (8.11) it can be shown [66] that in the potential $U^{+}(x)$ genuine quantum tunneling events "through" the potential barrier are rare above a so-called crossover temperature

$$
T_{c}^{+}=\frac{\hbar \mu^{+}}{2 \pi k_{B}},
$$

while for $T<T_{c}^{+}$tunneling yields the dominant contribution to the transition rates. An analogous crossover temperature $T_{c}^{-}$arises for the potential $U^{-}(x)$ which is typically not identical but rather close to $T_{c}^{+}$. With the definitions

$$
T_{c}^{\max }=\max \left\{T_{c}^{+}, T_{c}^{-}\right\}, \quad T_{c}^{\min }=\min \left\{T_{c}^{+}, T_{c}^{-}\right\}
$$

we now fix our last dimensionless quantity through $\Delta U^{\min } / k_{B} T_{c}^{\max }=10$. In this way, the weak noise condition (8.11) is safely fulfilled for $T \leq 2 T_{c}^{\max }$, i.e., up to temperatures well above both $T_{c}^{+}$and $T_{c}^{-}$. At the same time, the so-called semiclassical condition 66.

$$
\Delta U^{\min } \gg k_{B} T_{c}^{\max }
$$

can be taken for granted when evaluating the quantum mechanical transition rates (8.15) for all $T \leq 2 T_{c}^{\max }$. Specifically, the prefactor $A$ appearing in 8.15 ) can be evaluated within a saddle point approximation scheme [66] if the semiclassical condition (8.20) holds. Moreover, the implicit assumption in (8.12) that not only thermally activated barrier crossings are rare (see (8.11)) but also tunneling probabilities are small, is self-consistently fulfilled if 8.20 holds. For more details regarding the actual numerical calculation of those rates we refer to 161 .

Representative results 676, 677] for the above specified quantum ratchet model are depicted in figure 8.2. Shown are the current $\langle\dot{x}\rangle_{\mathrm{qm}}$ following from $(8.12)$ within the above sketched quantum mechanical treatment of the rates according to (8.15) together with the result $\langle\dot{x}\rangle_{\mathrm{cl}}$ that one would obtain by means of a purely classical calculation (formal classical limit $\hbar \rightarrow 0$ ) according to (8.13). The small dashed part in $\langle\dot{x}\rangle_{\mathrm{qm}}$ in a close vicinity of the crossover temperatures $T_{c}^{\max }$ and $T_{c}^{\min }$ from (8.19) signifies an increased uncertainty of the semiclassical rate theory in this temperature domain.

Our first observation is that even above $T_{c}^{\max }$, quantum effects may enhance the classical transport by more than a decade. They become negligible, that is, the physical classical limit is approached, only beyond several $T_{c}^{\max }$. In other words, significant quantum corrections of the classically predicted particle current set in already well above the cross-over temperature $T_{c}^{\max }$, where tunneling processes are still rare. 


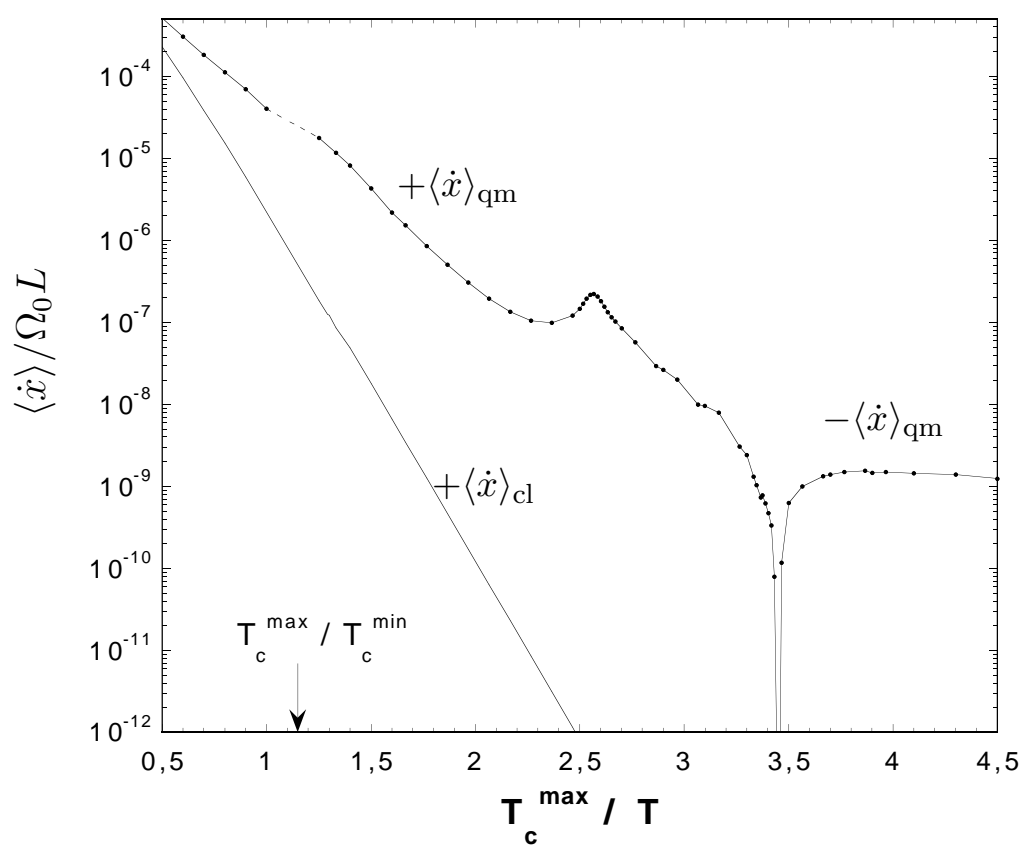

Figure 8.2: The classical steady state current $\langle\dot{x}\rangle_{\mathrm{cl}}$ and its quantum mechanical counterpart $\langle\dot{x}\rangle_{\text {qm }}$ for the ratchet potential from figure 8.1 in dimensionless units $\langle\dot{x}\rangle / L \Omega_{0}$. Note that in the present Arrhenius plot (logarithmic ordinate) the observed behavior of the quantum current near $T_{c}^{\max } / T=3.5$ is not the signature of a singularity but rather of a current inversion. Further worth mentioning features are the non-monotonicity of $\langle\dot{x}\rangle_{\mathrm{qm}}$ and that $\langle\dot{x}\rangle_{\mathrm{qm}}$ tends towards a finite limit when $T \rightarrow 0$.

(They can be associated to quantum effects other than genuine tunneling "through" a potential barrier.) With decreasing temperature, $T<T_{c}^{\min }$, quantum transport is even much more enhanced in comparison with the classical results. The most remarkable feature caused by the intriguing interplay between thermal noise and quantum tunneling is the inversion of the quantum current direction at very low temperatures [161, 420, 421, 676, 677, 681 683. Working within a formal a classical limit $(\hbar \rightarrow 0)$, such a reversal for adiabatically slow driving is ruled out. Finally, $\langle\dot{x}\rangle_{\mathrm{qm}}$ approaches a finite (negative) limit when $T \rightarrow 0$, implying a finite (positive) stopping force $f^{\text {f }}$ also at $T=0$. In contrast, the classical prediction $\langle\dot{x}\rangle_{\mathrm{cl}}$ remains positive but becomes arbitrarily small with decreasing $T$. A curious detail in figure 8.2 is the non-monotonicity of $\langle\dot{x}\rangle_{\mathrm{qm}}$ around $T_{c}^{\max } / T \simeq 2.5$, caused via (8.12) by a similar resonance-like $T$-dependence in the prefactor $A$ of one of the underlying quantum mechanical transition rates (8.15).

\footnotetext{
${ }^{4}$ Recalling the definition from section 2.6.2, the stopping force is that external force $F$ in $(3.1)$ which leads to a cancellation of the ratchet effect, i.e. $\langle\dot{x}\rangle=0$.
} 


\subsubsection{Tunneling induced current inversion}

The most remarkable result of the preceding subsection (see also figure 8.2) is the inversion of the current upon decreasing the temperature. On the other hand, within the formal classical limit $(\hbar \rightarrow 0)$ the current never changes its direction. Since at high temperatures the physical classical limit is approached, i.e. the formal classical limit provides a more and more accurate approximation for the true physical system, the temperature controlled current inversion represents a new signature of genuine quantum mechanical effects. In the following we provide a simple heuristic explanation of this finding [161, 421, 676].

As a first simplification, we exclusively focus in the exponentially leading contribution in the semiclassical rate expression (8.15), i.e. the sign of the current in (8.12) is given by that of $S_{l}^{-}-S_{r}^{+}$.

For sufficiently large temperatures, quantum mechanical effects become negligible and the exponentially leading part in (8.15) goes over into that of (8.13). Indeed, one can show [66, 96] that for $T>T_{c}^{\max }$ only the trivial extremizing paths $q(\tau) \equiv x_{b}$ in (8.16) exist for both potentials $U^{ \pm}(x)$, and thus we recover with (8.17) that $S_{r}^{+} / \hbar=$ $\Delta U_{r}^{+} / k_{B} T$ and $S_{l}^{-} / \hbar=\Delta U_{l}^{-} / k_{B} T$. In other words, the lower of the two barriers $\Delta U_{r}^{+}$ and $\Delta U_{l}^{-}$determines the direction of the current.

A second case for which the extremization of the action (8.16) can be readily carried out is the combined limit $T \rightarrow 0$ and $\eta \rightarrow 0$ (no heat bath), resulting in the familiar Gamow formula for the exponentially leading tunneling contribution in (8.17), namely

$$
S=2 \sqrt{2 m}\left|\int_{x_{0}}^{x_{1}} d q\left[U(q)-U\left(x_{0}\right)\right]^{1 / 2}\right| .
$$

As before, $x_{0}$ denotes a local minimum of $U(x)$ and $x_{1}$ is the first point beyond the considered potential barrier with the property that $U\left(x_{1}\right)=U\left(x_{0}\right)$. The absolute value in (8.21) is needed since $x_{1}<x_{0}$ for the escapes to the left, i.e., across $\Delta U_{l}^{-}$. Thus, the smaller of the two Gamow-factors $S_{r}^{+}$and $S_{l}^{-}$determines the direction of the current. Strictly speaking, by letting $\eta \rightarrow 0$ we of course violate our previously made assumption that deterministically running solutions should be ruled out. However, it is plausible that small but finite $\eta$ will exist for which our qualitative arguments can be adapted self-consistently.

From figure 8.1 one can see by naked eye that the activation energy barrier $\Delta U_{r}^{+}$ to proceed in the potential $U^{+}(x)$ from one local minimum to the neighboring local minimum to the right is smaller than the corresponding barrier $\Delta U_{l}^{-}$. Hence the current is positive for sufficiently large temperatures. In contrast, the fact that $S_{r}^{+}$is larger than $S_{l}^{-}$cannot definitely be read off by eye directly from figure 8.1 since the two quantities are rather similar, but it can be readily verified numerically. In other words, for very small $T$ indeed a negative current is predicted. A change of sign of the current at some intermediate temperature is thus a necessary consequence.

Things become even more obvious by considering instead of the smooth potential from figure 8.1 a stilized sawtooth profile local minimum $x_{0}=0$, the fact that $\Delta U_{r}^{+}<\Delta U_{l}^{-}$is read off immediately from figure 8.3. Denoting by $\lambda:=\left|x_{1}-x_{0}\right|$ the "tunneling-length", the Gamow-factor (8.21) takes

\footnotetext{
${ }^{5}$ For such a singular potential shape the $\mu$-factor in the crossover temperature 8.18 is no longer given by (8.14). Instead of changing the definition of $\mu$, one may also slightly smoothen out the singularities of the potential.
} 

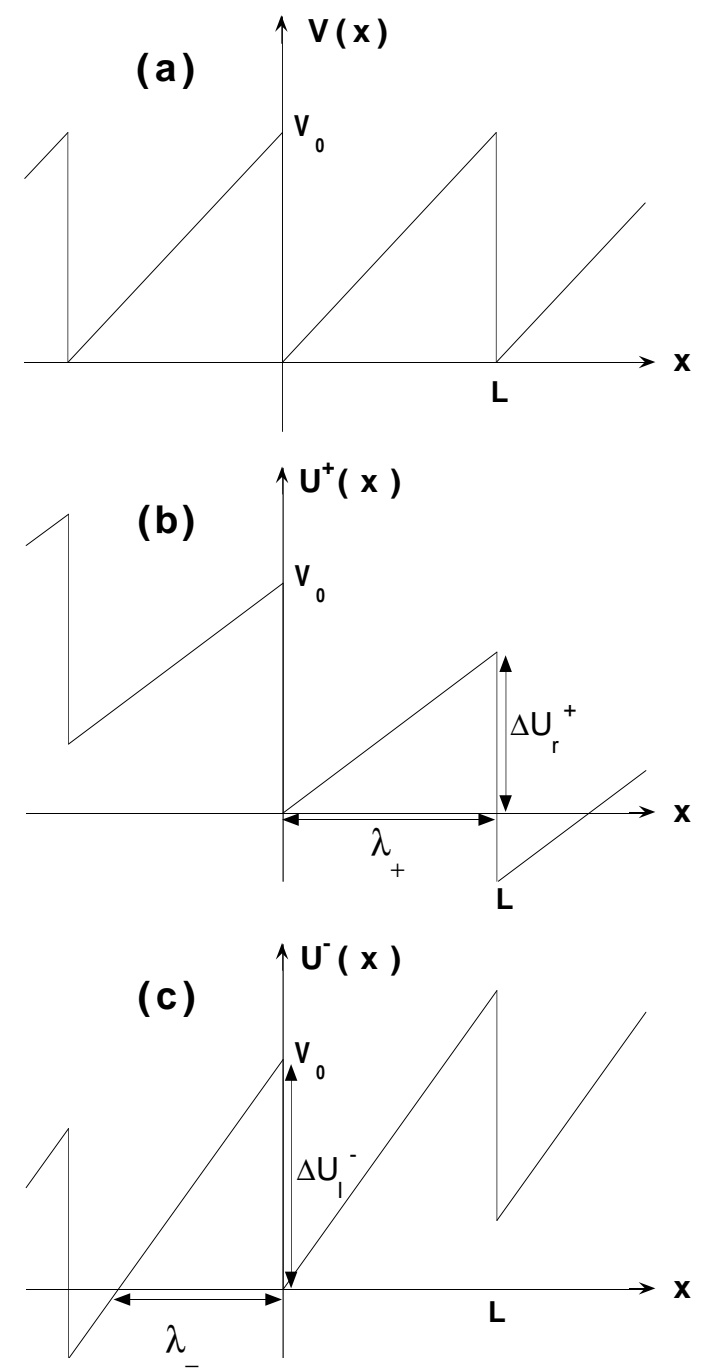

Figure 8.3: (a): Stylized sawtooth ratchet-potential $V(x)$ with spatial period $L$ and barrier height $V_{0}$. (b): The tilted ratchet potential $U^{+}(x)=V(x)-F x$ from 8.10) with the "tunneling-length" $\lambda_{+}$and the potential barrier $\Delta U_{r}^{+}$, relevant for the tunneling rate $k_{r}^{+}$from the local minimum $x_{0}=0$ to its neighboring local minimum to the right. (c): Same for the potential $U^{-}(x)=V(x)+F x$. [The depicted $F$-value is $V_{0} / 3 L$.] 
the simple form

$$
S=\frac{4}{3} \sqrt{2 m \Delta U} \lambda
$$

where indices $r, l$, and \pm have been omitted as usual. From figure 8.3 one reads off that $\lambda_{+}=L, \lambda_{-}=L /\left(1+L F / V_{0}\right), \Delta U_{l}^{-}=V_{0}$, and $\Delta U_{r}^{+}=V_{0}\left(1-L F / V_{0}\right)$, where we have assumed without loss of generality that $0 \leq F \leq V_{0} / L$. It readily follows that for small-to-moderate tilting forces $F \in\left[0,0.618 V_{0} / L\right]$ we have that $S_{r}^{+}>S_{l}^{-}$and the current is therefore negative.

In conclusion, the basic physical mechanism behind the opposite sign of the current at high and low temperatures is apparently rather simple and robust, suggesting that this feature should be very common in tilting quantum ratchet systems. Since the decrease of temperature is accompanied by a transition from thermally activated to tunneling dominated transport, the concomitant change of the transport direction may be considered as tunneling induced current inversion, see section 8.4.

\subsection{Beyond the adiabatic limit}

For an non-adiabatic tilting force $y(t)$ in (8.1) the determination of the average particle current is in general very difficult. An approximative analytical approach becomes possible within a so-called tight-binding model description. The starting point consists in the observation that the first two terms on the right hand side of (8.1) define a timeindependent particle dynamics in a periodic potential and can thus be treated within the standard Bloch-theory for independent (quasi-)particles in a one-dimensional lattice 468. Under the assumptions that both the external tilting force $y(t)$ and the thermal fluctuations of the environment, entering through the last two terms in (8.1), are sufficiently weak, one can focus on a single-band truncation of the problem, i.e., the Hilbert-space accessible to the particle is spanned solely by the Bloch-states of the lowest energy-band. Especially, both the thermal energy $k_{B} T$ and the energy $\hbar \omega_{c}$ associated to the cutoff in (8.8) have to be restricted to values much smaller than the excitation energy into the second band (or the continuum). Upon going over from these Bloch-states of the lowest band to a new basis $\{|n\rangle\}_{n=-\infty}^{\infty}$ of so-called localizedor Wannier-states 468], the truncated model Hamiltonian (8.1) takes the standard single-band tight-binding form [684]

$$
\mathbf{H}(t)=-\frac{\hbar \Delta}{2} \sum_{n=-\infty}^{\infty}(|n\rangle\langle n+1|+| n+1\rangle\langle n|)-\mathbf{x} y(t)+\mathbf{H}_{B},
$$

where both in $(8.23)$ and $(8.2)$ the operator $\mathbf{x}$ is defined as

$$
\mathbf{x}:=L \sum_{n=-\infty}^{\infty} n|n\rangle\langle n| .
$$

The quantity $\hbar \Delta$ in (8.23) is the so-called tunneling coupling energy between neighboring potential minima. In principle, its explicit value can be determined from the Bloch-states and the potential $V(x)$ 468]. Alternatively, the tunneling coupling energy may be considered as an adjustable model parameter. An additional approximation implicit in $(8.23)$ is the assumption that only tunneling between neighboring potential 
minima of $V(x)$ plays an appreciable role. In other words, so-called coherent tunneling (co-tunneling) is neglected.

By construction, the single-band tight-binding model (8.23) cannot capture thermally activated transport across the energy barriers between neighboring minima of $V(x)$; its validity is restricted to quantum mechanical tunneling processes at low energies and temperatures. Furthermore, the model does not exhibit any traces of a possible asymmetry in the periodic potential $V(x)$. One is therefore restricted to effectively symmetric potentials $V(x)$ and a ratchet effect may only be studied within an asymmetrically tilting ratchet scheme (see section 5.8). Besides these restrictions, the tight-binding model also goes beyond the approach from sections 8.2, 8.2.1 in that the semiclassical condition $(8.18)$ is not required and the tilting force $y(t)$ needs not be adiabatically slow (see below). In this sense, the approaches from sections 8.2, 8.2.1 and of the present section are complementary.

A non-adiabatically tilting quantum ratchet within the above single-band tightbinding approximation has been considered in 685] for a rather general class of unbiased, asymmetric random drivings $y(t)$, including asymmetric dichotomous noise as a special case. In the absence of the heat bath $\mathbf{H}_{B}$ in (8.23), the average particle current is found to vanish in all cases (for the same model, but with a periodic driving $y(t)$, see also [166, 167]). In the presence of the heat bath, the occurrence of a finite current is generically observed. Current inversions upon variation of different model parameters are also reported. Especially, such an inversion may occur when the temperature is changed, which, for reasons detailed above, cannot be explained by the heuristic argument from section 8.3 and thus represent a genuine feature of the non-adiabatic driving. Regarding a more detailed discussion of the effective diffusion coefficient (3.6) within this model we refer to the original paper [685].

The same model, but with an asymmetric periodic driving $y(t)$ of the harmonic mixing form (5.58) has been addressed in 686]. The emerging quantum current exhibits multiple reversals, characteristic for the non-adiabatic nature of the driving, and a stochastic resonance-like, bell-shaped behavior upon variation of the temperature. Via control of the phase and the amplitudes of the driving signal (5.58) it is furthermore possible to selectively control the magnitude of both the quantum current and diffusion, as well as the current direction. For further theoretical and experimental works along related lines see [166, 167, 384 388] and references therein?.

While the rich behavior of the single-band tight-binding ratchet model can be obtained by means of sophisticated analytical approximations 685,686 which go beyond our present scope, simple intuitive explanations can usually not be given.

Generalizations of the single-band tight-binding model (8.23) have been addressed in 688 690. The main new ingredient is an extra "potential"-term $\mathbf{H}_{V}$ of the form

$$
\mathbf{H}_{V}=\sum_{n=-\infty}^{\infty}|n\rangle\langle n| V_{n \bmod N}[1+f(t)]
$$

on the right hand side of (8.23), reminiscent of a spatially discretized, asymmetric ratchet potential with period $N \geq 3$. The case with $y(t) \equiv 0$ in (8.23), corresponding to a fluctuating potential ratchet, has been treated in [688, 690]. The opposite case with $f(t) \equiv 0$ but again with an adiabatically slow, symmetric rocking force $y(t)$ has been

\footnotetext{
${ }^{6}$ Closer inspection indicates [166, 687] that the conclusions from [388] in the case of a dissipationless (collisionless) single-band model are at most valid for very special (non-generic) initial conditions.
} 
addressed in 689. At first glance, such an extra term 8.25) in order to upgrade the single-band tight-binding model (8.23) into a veritable ratchet system with a broken spatial symmetry is indeed suggestive. However, the present author was only able to figure out very artificial actual physical situations which may be captured by such a model with a non-trivial term (8.25). In particular, due to the nearest neighbor hopping term in (8.23), such a description clearly cannot properly account for more than one single band.

Further quantum ratchet works, some of which go beyond the adiabatic limit, and which are not based on the single-band tight-binding approximation (8.23) of the original dynamics (8.1), are 496, 548, 690, 691]. However, the present author finds these studies questionable with respect to the conceptual basis and/or the technical methodology. A quantum Smoluchowski-Feynman-type model (equilibrium system) has been investigated on the basis of a widely used standard approximation in 663] and further references therein. The observed appearance of a ratchet effect in such an equilibrium model underlines once more the warning at the beginning of section 8.1 that even well-established ad hoc approximations for a quantum thermal environment may easily lead to inconsistencies with fundamental principles of statistical mechanics.

For a periodic driving $y(t)$ and in the absence of the heat bath $\mathbf{H}_{B}$ in (8.1) the quantum mechanical counterpart of the Hamiltonian rocking ratchet model from equation (5.34) in section 5.4 is recovered. Within a single-band tight-binding approximation (8.23) this type of model has been solved in closed analytical form in 166, 167, 685. Though the chaotic features of the classical counterpart cannot be captured in this way, a dependence of the current on the initial conditions is found 166, 167 which is quite similar to the classical results from [165], while strongly non-classical features [685] arise in the presence of a finite static tilt $F$ (i.e. $y(t)$ in (8.23) is replaced by $y(t)+F$ ). A first step into the direction of a chaotic (Hamiltonian) quantum ratchet system has been taken in 4113, 414 and 692] with the main focus on the semiclassical regime and on mesoscopic electron billiard devices, respectively, see also section 5.4 for the classical limit.

\subsection{Experimental quantum ratchet systems}

As a first candidate for an experimental realization of a quantum ratchet we consider the SQUID rocking ratchet model [357] from equation (5.38). As argued at the beginning of this chapter, this stochastic dynamics (5.38) as it stands represents a classical ratchet system [94]. The question of how to properly "quantize" such a "classical" dynamics, which itself arises as an effective description of characteristic quantum effects, has been discussed extensively in the literature, see [94, 669] and references therein. Leaving aside devices which contain ultra small tunnel junctions, ample theoretical [669, 693 695] as well as experimental (see references in [66]) justification has been given that, after proper renaming of symbols, equations (8.1), (8.8) provide the basis for an adequate quantum mechanical extension of the classical model (5.38) when the temperature is decreased below a few times $T_{c}^{\max }$ from (8.19). Conceptionally, it is interesting to note 94] that we are dealing here with quantum effects which manifest themselves via the macroscopic phase-variable $\varphi$. In other words, the observation of transport properties characteristic for a quantum ratchet is not necessarily restricted to the realm of microscopic systems. So far, an actual experimental realization of a SQUID ratchet system [25, 26, 182, 183] is only available for the two- 
dimensional modification (5.52), (5.53) of the archetypal rocking ratchet setup (5.38). While the experiment from [182, 183] works with high- $T_{c}$ SQUIDS at temperatures too large to see any traces of quantum mechanical tunneling of the phase $\varphi$, an analogous experiment with conventional superconductors is presently under construction, with the intention to reveal such quantum mechanical effects.

A second potential realization of a tilting quantum ratchet system is based on the motion of ultracold atoms in the presence of standing electromagnetic waves, creating a ratchet potential through the radiation-pressure forces of the counterpropagating light beams 696 700. For sufficiently weak potentials and low temperatures, quantum effects will clearly play a dominant role in the atomic motion and may be roughly captured by a model like in (8.1). Especially, the tilting force may be created by exploiting the mapping of the model onto an improper traveling potential ratchet from section 4.4.2. The corresponding accelerating optical potentials have been experimentally realized e.g. in 700. As detailed in section 4.5.2, a somewhat related system has indeed been experimentally studied in [339]. Due to the remaining considerable differences between this system and the theoretical model (8.1), a direct comparison is, however, not possible.

A third promising class of experimental tilting quantum ratchet devices are semiconductor heterostructures. The lacking periodicity of a single diode (n-p juction) can be readily remedied, in the simplest case by connecting identical diodes by normal conducting wires. Similarly as in the above discussed case of SQUID ratchets, such a simple array of diodes realizes a classical ratchet system in so far as (at the usual working temperatures) the essential transport processes across the junctions are governed by classical thermal diffusion rather than quantum mechanical tunneling (see also sections 2.9, 5.2, and 6.1). Closely related devices are spatially periodic semiconductor superlattices. Examples with broken spatial symmetry (so-called sawtooth superlattices) have been experimentally realized since long [701, 702 but have never been studied so far from the viewpoint of the ratchet effect. Heterostructures consisting of alternating layers of GaAs and AlGaAs in quantum mechanically dominated temperature regimes have been experimentally explored e.g. in [703 705]. The motion of a (quasi-) particle (dressed electron) in such a superlattice may be roughly described by an effective, one-dimensional model of the form (8.1), where the heat bath takes into account the effects of the crystal phonons 685, 686, 704, 705]. In the simplest case of a semiconductor superlattice with only two different alternating layers, a symmetric periodic potential $V(x)$ in (8.1) arises, thus the asymmetrically tilting quantum ratchet scheme from section 8.3 has to be employed. The quantitative estimates from 686 furthermore show that the one-band tight-binding model (8.23) may be a valid approximation for a typical experimental setting 703 705. Moreover, if the driving $y(t)$ is provided by the usual electromagnetic waves in the THz-regime, one is indeed dealing with the non-adiabatic regime from section 8.3. On the other hand, the semiclassical theory from section 8.2 cannot be applied to such an experimental situation not only because the driving is not adiabatically slow, but also since the semiclassical condition (8.18) is typically not satisfied.

Important progress towards an adiabatically rocking quantum ratchet in onedimensional Josephson junction arrays, consisting of three "cells" (effective periods) with broken spatial symmetry, has been achieved very recently in 706]: somewhat similar as in the systems from section 5.3.3, but operating in the quantum mechanical regime, the voltage due to the dynamical response of the vortices (directed transport 
of quasi particles) against an applied bias current exhibits an asymmetry when the sign of this bias is inverted, cf. figure 2.4. In particular, the theoretically expected asymptotic temperature independence of the effect in the deep quantum cold is experimentally recovered.

A molecular rectifier for electrons, combining the quantum ratchet with the Coulomb blockade effect, has been proposed in 428]. For additional experiments which may be considered to some extent as quantum ratchet systems we also refer to the applications of the genuine traveling potential ratchet scheme discussed in section 4.4.1.

We close this section with the experimental realization of an adiabatically rocking quantum ratchet by Linke and colleagues on the basis of a quantum dot array with broken spatial symmetry. We skip the preliminary experiment on ac-driven electron transport through a single triangular shaped quantum dot 420, 707 711 (see also 429,) and immediately turn to the exploration of an entire array of such triangular $\operatorname{dots}[421,682,683,712,714$.

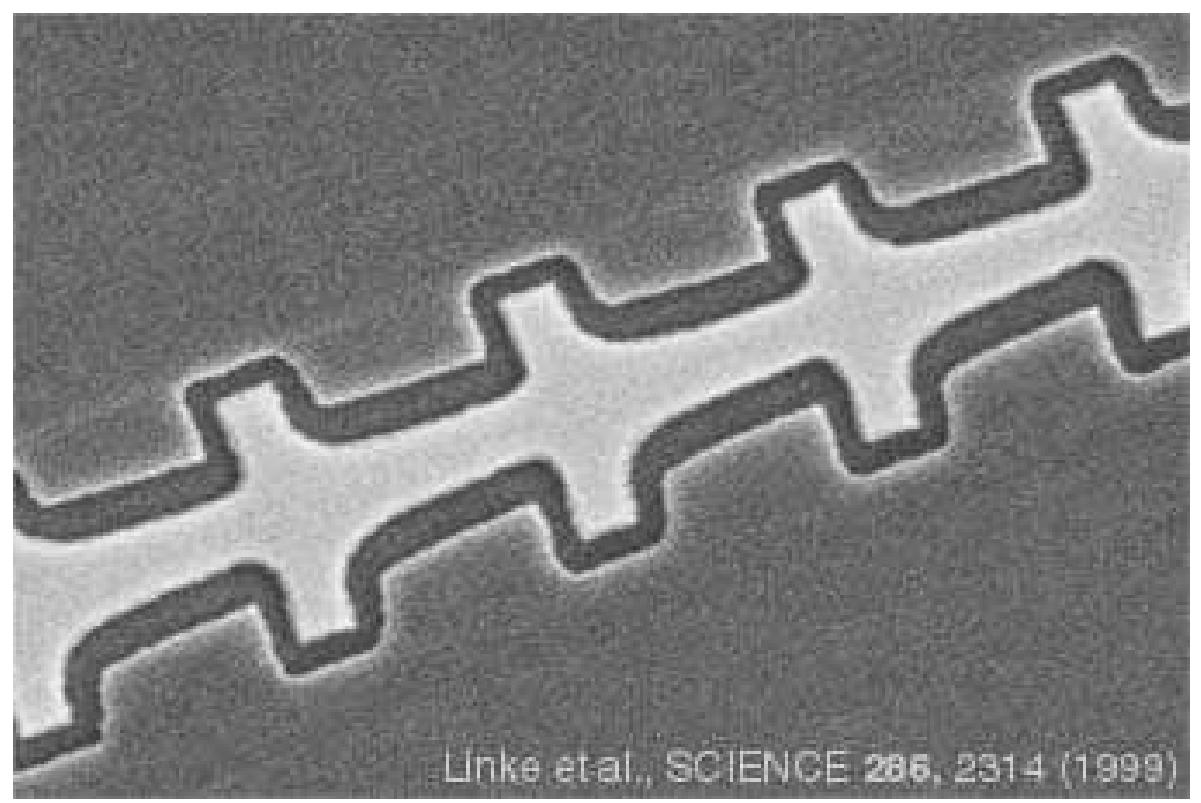

Figure 8.4: Scanning electron micrograph of an array of triangular shaped quantum dots, etched from a GaAs/AlGaAs semiconductor heterostructure. The depicted top view defines the $x-y$-plane accessible to the two-dimensional conducting electron gas. The etched areas (dark regions) are insulating domains for the electrons. Shown are 4 out of the 10 triangles used in the actual experiment [712]. The period $L$ of the triangles is about $1.2 \mu \mathrm{m}$.

The basic setup [712] is depicted in figure 8.4: A two-dimensional conducting electron gas is constricted by two insulating boundary-regions (dark areas in figure 8.4). In other words, the "conducting channel" along the $x$-axis is laterally confined to a width of about $1 \mu \mathrm{m}$. Roughly speaking, the corresponding lateral confinement energy creates an effective ratchet-shaped potential $V(x)$ for the particle dynamics along the $x$-axis of a qualitatively similar character as in figure 8.1. The two "side gates" in figure 8.4 allow one to externally modify this effective potential by putting them on different electrical potentials. The actual rocking force $y(t)$ is created by applying an ac-voltage along the $x$-axis, periodically switching between the two values $\pm F$, with a 
typical voltage $F$ of about $1 \mathrm{mV}$. The driving frequency of $191 \mathrm{~Hz}$ used in the experiment is definitely deep within the adiabatic regime. The "bottlenecks" which connect neighboring triangles in figure 8.4 are chosen such that quantum tunneling dominates at low temperatures, while for higher temperatures the conduction electrons can also substantially proceed by way of thermal activation across the corresponding effective potential barriers.

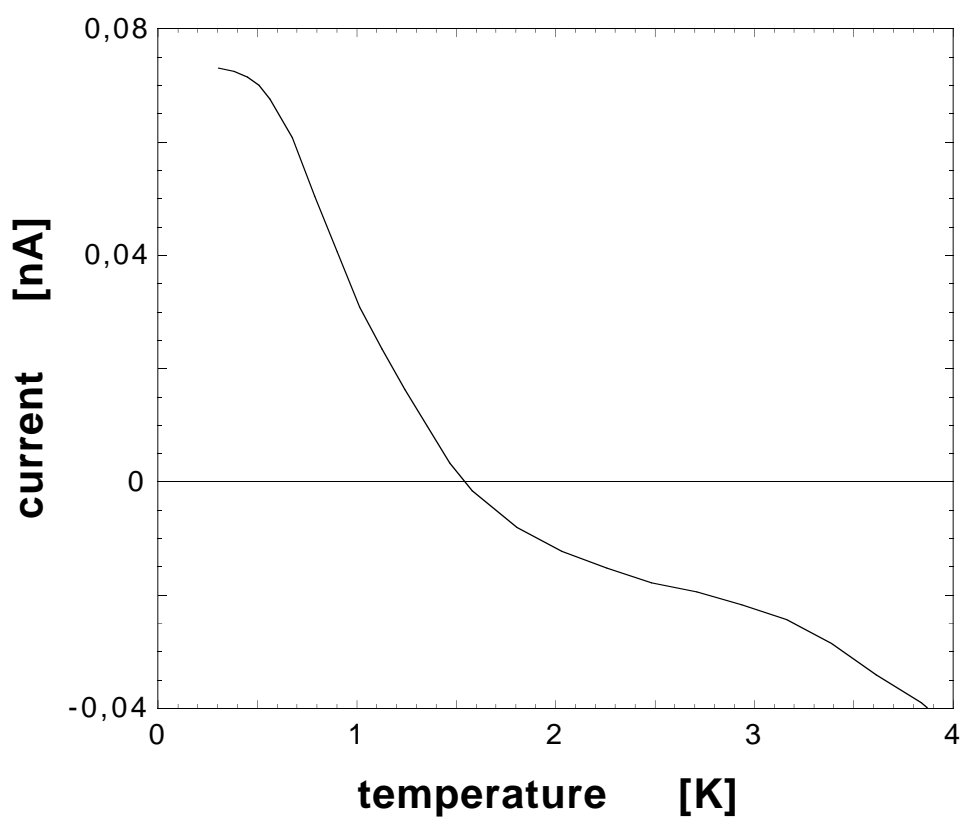

Figure 8.5: Electrical current $I=-e\langle\dot{x}\rangle$ along the quantum dot array from figure 8.4 versus temperature for an unbiased rocking voltage $y(t)$ which periodically jumps between $\pm 1 m V$ at a frequency of $191 \mathrm{~Hz}$ [12].

The measured [712 current through the quantum dot array as a function of temperature is exemplified in figure 8.5. The two main features theoretically predicted in section 8.2 are clearly reproduced, namely a current inversion upon decreasing the temperature and a saturation of the current as temperature approaches absolute zero[. Though a model along the lines of (8.1) is obviously a very crude description of the experimental situation, the basic features and thus the heuristic explanation of the current inversion from section 8.2.1, are apparently still qualitatively correct. Note that also the direction of the current is in agreement with figure 8.2 by taking into account that the relevant effective potential for the experiment is of the same qualitative shape as in figure 8.1 and that the electrical current is opposite to the particle current for the negatively charged electrons. For a somewhat more realistic theoretical model, which also reproduces the main qualitative features of the experiment, we refer to 712,713 .

Based on the observation from section 8.2.1, namely that "cold" and "hot" particles move in opposite directions (as long as their individual "temperatures" (kinetic energy) change sufficiently little), an interesting idea is 564,715 to apply the above quantum rocking ratchet setup in the absence of a net particle transport, i.e. operat-

\footnotetext{
${ }^{7}$ Note that figure 8.2 is an Arrhenius plot $(\log \langle\dot{x}\rangle$ versus $1 / T)$, while figure 8.5 depicts the bare quantities "electrical current" versus "temperature".
} 
ing at the current inversion point, for "cooling" purposes 8 . However, the quantitative analysis of the experiment in [712] shows that the heating due to the external rocking force exceeds the cooling effect due to the above separation of particles with different temperatures [715]. On the other hand, assuming the existence of "ideal filters", which let pass in both directions only particles with one specific energy, it is possible to modify the original setup such that it can act either as refrigerator or as heat engine arbitrarily close to the maximal Carnot efficiency [564]. In contrast to the standard framework for considerations on the efficiency of particle transport from section 6.9, here a zero particle current situation is addressed, and the relevant mechanical work is now associated with the external driving force. In other words, the particle motion is now considered as an internal part of the engine under consideration, and no longer as the resulting effect of the engine.

\footnotetext{
${ }^{8}$ Of foremost interest in this context are "single-period setups" (e.g. a single triangular quantum dot) in contact with two electron-reservoirs at either the same or different temperatures.
} 


\section{Chapter 9}

\section{Collective effects}

At the focus of this chapter are collective effects that arise when several copies of "single" classical ratchet systems, as considered in extenso in the previous chapters 2-6, start to interact with each other.

Accordingly, the general working model (3.1) goes over into $N$ coupled stochastic differential equations of the form

$$
\eta \dot{x}_{i}(t)=-V^{\prime}\left(x_{i}(t), f_{i}(t)\right)+y_{i}(t)+F+\xi_{i}(t)-\frac{\partial \psi\left(x_{1}(t), \ldots, x_{N}(t)\right)}{\partial x_{i}}
$$

with $i=1, \ldots, N$. The last term accounts for the interaction through an interaction potential $\psi$ which is assumed to be spatially homogeneous and inversion symmetric, i.e. no preferential direction is introduced through the interaction. The assumption of a thermal equilibrium environment implies that the thermal noises $\xi_{i}(t)$ are mutually independent Gaussian white noises with correlation

$$
\left\langle\xi_{i}(t) \xi_{j}(s)\right\rangle=2 \eta k_{B} T \delta_{i j} \delta(t-s)
$$

The drivings $f_{i}(t)$ and/or $y_{i}(t)$ are usually assumed to be either mutually independent random processes or equal to the same periodic function for all $i$. In any case, these drivings as well as the interaction $\psi$ in (9.1) have to respect the equivalence of all the "single particles" $i$. Therefore, the average particle current $\langle\dot{x}\rangle$ from (3.5) will be the same for each particle $i$ and consequently independent of whether or not an additional average over $i$ is performed. The case of foremost interest is usually the zero load $(F=0)$ situation, but also the response when a finite $F$ is acting will lead to quite remarkable observations in section 9.2 .

We recall that various examples with $N=2$ interacting systems (9.1) have been discussed already in sections 5.5, 6.5, and 7.6. In the present chapter, our main interest will concern collective effects in the case of a large number $N \rightarrow \infty$ of interacting systems (thermodynamic limit). In doing so, two basic types of questions can be addressed. First, one may consider cases for which already in the absence of the interaction in (9.1) each single system exhibits a ratchet effect, i.e. both, thermal

\footnotetext{
${ }^{1}$ A deterministic collective model which does not fit into this general framework is due to [716, 717]: It works for $N \geq 3$ particles with finite mass $m$ in a static, not necessarily asymmetric potential $V(x)$. A worm-like deterministic motion is generated by active changes of the interaction in a wavelike manner along the chain of particles $x_{i}(t)$. A similar model with non-Newtonian interaction forces $($ actio $\neq$ reactio) is due to 718 . Moreover, reaction-diffusion model for interacting Brownian motors has been discussed in section 6.5 .
} 
equilibrium and spatial symmetry are broken. In such a case, one may study the modification of the current $\langle\dot{x}\rangle$ in magnitude and possibly even in sign when the interaction is included. A survey of such explorations will be presented in section 9.1.

A second type of questions regards genuine collective effects, namely spontaneous ergodicity breaking, entailing phase transitions, the coexistence of different (meta-) stable phases, and hysteretic behavior in response to the variation of certain parameters. While all these collective phenomena are well known also in equilibrium systems, the second law of thermodynamics precludes a finite particle current in such systems even if their spatial symmetry is broken. We thus focus on interacting systems (9.1) out of equilibrium, which for the usual interactions is the case if and only if already the uncoupled systems (9.1) are out of equilibrium. Such genuine non-equilibrium collective effects have already been encountered in the context of the Huxley-Jülicher-Prost model for cooperating molecular motors in section 7.4.4. There, the main emphasize was put on systems with a built-in spatial asymmetry already of the single (uncoupled) systems in (9.1), which is then inherited by the coupled model. In contrast, in section 9.2 we will address coupled non-equilibrium systems (9.1) which are fully symmetric under spatial inversion. The essential idea is then that instead of a built-in asymmetry, a perfectly symmetric system may create the asymmetry, which is necessary for the manifestation of a ratchet effect, by itself, namely through spontaneous symmetry breaking. While the occurrence of such a "spontaneous current" has been pointed out already for a spatially symmetric special case of the Jülicher-Prost model in [557], we will focus in section 9.2 on a simpler model which admits a partial analytical treatment and exhibits additional, quite remarkable collective non-equilibrium features.

We close with two remarks: First, the subject under study in this chapter is intimately related with many other topics, like for instance non-equilibrium phase transitions, reaction-diffusion systems, pattern formation, driven diffusive systems, FrenkelKontorova models, Josephson junction arrays, sine-Gordon equations, and coupled phase oscillators. A detailed discussion of any of these adjacent topics goes, however, beyond the scope of our present review. Second, while we feel that very interesting and unexpected theoretical discoveries are still to come, on the experimental side the field is even more so at a very underdeveloped stage.

\subsection{Coupled ratchets}

In this section we review investigations of $N \rightarrow \infty$ coupled ratchet systems in the case that each single particle $i$ exhibits a ratchet effects already in the absence of the interaction in (9.1). For related discussions of models for cooperative molecular motors we also refer to section 7.4. Throughout this section, we restrict ourselves to the case $F=0$ in $(9.1)$ and to potentials $V(x)$ with a broken symmetry, i.e. ratchet potentials, as exemplified by figures 2.2 and 4.1 .

The case of interacting rocking ratchets

$$
\eta \dot{x}_{i}(t)=-V^{\prime}\left(x_{i}(t)\right)+y(t)+\xi_{i}(t)+I_{b}\left(x_{i+1}(t), x_{i}(t), x_{i-1}(t)\right)
$$

with a hard core repulsive interaction $I_{b}$ such as to guarantee $x_{i+1}(t)>x_{i}(t)+b$ for all $i$ and $t$ has been explored in [719]. Pictorially speaking, all particles are thus moving in the same one-dimensional, periodically rocked ratchet potential $V(x)-x y(t)$ and they have a finite extension $b$ which sets a lower limit for their mutual distance. 
The central (numerical) finding in [719] is a current inversion upon variation of the average density of particles along the $x$-axis. This inversion is robust against various modifications, especially of the driving $y(t)$ in (9.3) [e.g. stochastic instead of periodic, or with small, $i$-dependent variations of the driving-period $\mathcal{T}$ ] and implies according to section 3.6 analogous inversions upon variation of practically any other parameter of the model (9.3). For adiabatically slow driving $y(t)$ and simultaneously almost densely packed particles, an analytical treatment is possible, revealing an extremely complex dependence of the current upon the particle extension $b$. Somewhat similarly as in the Jülcher-Prost model [557], also in the present case the magnitude of the current $\langle\dot{x}\rangle$ depends sensitively on whether the spatial period $L$ is commensurate or not with the average interparticle distance $\left\langle x_{i+1}(t)-x_{i}(t)\right\rangle$. Such effects may become practically relevant for separating particles at high densities e.g. according to the drift ratchet scheme from section 6.6. A related, spatially discrete model with an adiabatically slow driving has been considered in [519], thus establishing contact with the methods and concepts of so-called driven diffusive systems 607.

A second basic model consists of a chain of linearly coupled fluctuating force ratchets

$$
\eta \dot{x}_{i}(t)=-V^{\prime}\left(x_{i}(t)\right)+y_{i}(t)+\xi_{i}(t)+\kappa\left[x_{i+1}(t)-2 x_{i}(t)+x_{i-1}(t)\right],
$$

where $\kappa$ is the spring constant (interaction strength) and $y_{i}(t)$ are independent Ornstein-Uhlenbeck noise sources (cf. equations (3.13), (3.15)). In the continuum limit, one obtains a sine-Gordon type model, which has been analyzed by means of the sophisticated analytical machinery in this field in [720. The main result is the appearance of a ratchet effect in the form of a stationary directed transport of kinks and antikinks in opposite directions. As a rule, the kink and hence the entire particle chain move into the same direction as in the uncoupled, Ornstein-Uhlenbeck noise driven fluctuating force ratchet (cf. section 5.1), however with a highly non-trivial modification of the quantitative behavior of the current. Similar results for models of the type (9.4) have been reached also in 721 724 and for an analogous coupled temperature ratchet model in 482. Possible applications include the dynamics of dislocations in solids, solitonic fluxes in long Josephson junction arrays and magnetically ordered crystals, and models for friction and stick-slip motion such as the Frenkel-Kontorova model. For related studies in the context of coupled Josephson junction arrays see also section 5.3.3.

Next we turn to the interacting on-off ratchet counterpart of (9.3), i.e.

$$
\eta \dot{x}_{i}(t)=-V^{\prime}\left(x_{i}(t)\right)\left[1+f_{i}(t)\right]+\xi_{i}(t)+I_{b}\left(x_{i+1}(t), x_{i}(t), x_{i-1}(t)\right)
$$

with $f_{i}(t) \in\{ \pm 1\}$. In this case [225], the direction of the particle current $\langle\dot{x}\rangle$ may even change many times as the density of particles is varied. For high particle densities and slow on-off cycles, an extremely complex dependence of $\langle\dot{x}\rangle$ on the particle size $b$ similarly as for the model (9.3) is recovered. Such effects clearly become relevant for the various experiments from section 4.2.1 at high particle densities. An experiment which may be considered to some extent as related to the theoretical model (9.5) has been realized in [725, 726]. In this work, the horizontal transport of granular particles in a vertically vibrated system, whose base has a ratchet-shaped profile, has been measured . The resulting material flow exhibits current inversions and other complex

\footnotetext{
${ }^{2}$ A computer animation (Java applet) which graphically visualizes a somewhat related effect is available on the internet under 727].
} 
collective behavior as a function of the particle density and the driving frequency, displaying a rough qualitative similarity with the theoretical model (9.5).

A coupled rocking ratchet model, but in contrast to (9.3) with a global, Kuramototype interaction 629, 728, 729] with the same period $L$ as the ratchet potential $V(x)$, i.e.

$$
\eta \dot{x}_{i}(t)=-V^{\prime}\left(x_{i}(t)\right)+y(t)+\xi_{i}(t)+\frac{K}{N} \sum_{j=1}^{N} \sin \left(\frac{2 \pi}{L}\left[x_{j}(t)-x_{i}(t)\right]\right)
$$

has been addressed in [730]. Upon increasing the coupling strength $K$, the current may change direction and moreover the effect of the noise becomes weaker and weaker: For $K \rightarrow \infty$ all particles in (9.6) are lumped (modulo $L$ ) into one single effective "superparticle" subjected to an effectively deterministic single-particle rocking ratchet dynamics like in section 5.3. The existence of current inversions upon variation of other model parameters than the coupling strength immediately follows from section 3.6. Considering that a single particle $(N=1)$ rocking ratchet can be realized by means of three Josephson junctions (see equation (5.38)), the coupled model (9.6) may well be of relevance for Josephson junction arrays [731].

Universal properties of particle density fluctuations at long wavelengths and times for a large class of short-range interaction ratchet models like for instance in (9.3), (9.5) have been revealed in 732. More precisely, the steady state density-density correlation function exhibits dynamical scaling according to the Kadar-Parisi-Zhang universality class [732].

\subsection{Genuine collective effects}

For non-interacting periodic systems, the basic result of the previous chapters $2-6$ is that necessary, and generically also sufficient conditions for the occurrence of directed transport are that the system is out of thermal equilibrium and that its spatial symmetry is broken. The essential idea of this section is to abandon the latter condition of a built-in asymmetry. Instead, the system may create an asymmetry by itself as a collective effect, namely by way of spontaneous symmetry breaking. As a consequence, according to Curie's principle, a collective ratchet effect in the form of a "spontaneous current" is then expected It. It turns out that this idea can indeed be realized, and in fact even in several different ways [14, 530, 557, 558, 622, 733 737]. Here, we will focus on a particularly simple example of globally coupled fluctuating potential ratchets [530,733, 736]. We finally remark that the appearance of a "spontaneous current" has also been predicted in a rather different theoretical mean field model for driven semiconductor superlattices in [738,739.

\footnotetext{
${ }^{3} \mathrm{~A}$ nearest neighbor instead of the global coupling in $(9.6)$ may then be a more realistic choice. Such a modification is, however, not expected to change the basic qualitative features of the model (at least in $d \geq 2$ dimensions), see also figures 9.2 and 9.3 below.

${ }^{4}$ In contrast to "permanent currents", appearing for instance in mesoscopic rings at thermal equilibrium, the "spontaneous currents" which we have here in mind can be exploited to do useful work and are moreover a purely classical phenomenon.
} 


\subsubsection{Model}

As a combination of the fluctuating potential ratchet scheme from (4.11) and of our general working model for interacting systems (9.1) we take as starting point the following set of $i=1, \ldots, N$ coupled stochastic equations

$$
\dot{x}_{i}(t)=-V^{\prime}\left(x_{i}(t)\right)\left[1+f_{i}(t)\right]+\xi_{i}(t)+\frac{K}{N} \sum_{j=1}^{N} \sin \left(x_{j}(t)-x_{i}(t)\right) .
$$

For the sake of simplicity only, we consider a Kuramoto-type, sinusoidal global coupling 629, 728, 729], and we will restrict ourselves to attractive interactions $K>0$. Furthermore, we have adopted dimensionless units (see section A.4 in Appendix A) with

$$
\eta=k_{B}=1, L=2 \pi \text {. }
$$

[For esthetical reasons we will often continue to use the symbol $L$.] In particular, the potential $V(x)$ and the interaction respect the same periodicity $L=2 \pi$. However, in contrast to "conventional" fluctuating potential ratchets without interaction (see section 4.3), we exclude any built-in spatial asymmetry of the system (9.7), which can be achieved if the potential $V(x)$ respects the symmetry condition

$$
V(-x)=V(x)
$$

independently of any further properties of $f_{i}(t)$, see the discussion below (3.23). Finally, in view of the analytic tractability in the absence of interaction (see section 4.3.2) we specialize to potential fluctuations $f_{i}(t)$ which are given by independent OrnsteinUhlenbeck processes (3.13), (3.15) of strength

$$
\int_{-\infty}^{\infty} d t\left\langle f_{i}(t) f_{j}(s)\right\rangle=2 Q \delta_{i j}
$$

(cf. equation (4.14) ) and a negligibly small correlation time $\tau$ in comparison to all the other relevant time scales of the system.

\subsubsection{Spontaneous symmetry breaking}

In this section, we first present a somewhat formal analytical demonstration of the existence of spontaneous symmetry breaking for a system (9.7) in the thermodynamic limit $N \rightarrow \infty$, followed quantitative numerical illustrations and an intuitive explanation of the basic physical mechanism at work.

The main collective features of (9.7) are captured by the particle density

$$
P(x, t):=\frac{1}{N} \sum_{i=1}^{N} \delta\left(x-x_{i}(t)\right) .
$$

In contrast to the definition for non-interacting systems in (2.9), the average over the noise is omitted in (9.11) and instead an average over the particles $i$ is included.

\footnotetext{
${ }^{5}$ Mathematically, we avoid in this way additional complications due to incommensurability effects. Physically, this assumption is especially natural if the state variables $x_{i}$ are originally of a phase-like nature, see section 3.4.2. Some generalizations will be addresses in section 9.2 .5 below.
} 
Being an intensive quantity, $P(x, t)$ becomes independent of the specific realization of the noises $\xi^{0} \xi_{i}(t)$ and $f_{i}(t)$ when $N \rightarrow \infty$ (self-averaging), as demonstrated in detail in 740743 . In other words, it does actually not matter whether we consider an average over the noise as included or not in the definition of $P(x, t)$ in (9.11). Finally, we go over to the reduced density $\hat{P}(x, t)$ as usual, cf. section 2.4 .

By rewriting the interaction term in (9.7) as $K\left[S \cos \left(x_{i}(t)\right)-C \sin \left(x_{i}(t)\right)\right]$, where]

$$
S:=\int_{-L / 2}^{L / 2} d x \hat{P}(x, t) \sin x \quad C:=\int_{-L / 2}^{L / 2} d x \hat{P}(x, t) \cos x
$$

the dynamics of each particle (9.7) is exactly of the type which we have considered in section 4.3.2. By summing the corresponding single particle Fokker-Planck equations (2.17), (4.25) according to (9.11) one recovers

$$
\begin{aligned}
& \frac{\partial}{\partial t} \hat{P}(x, t)=\frac{\partial}{\partial x}\left\{\hat{V}^{\prime}(x)+g(x) \frac{\partial}{\partial x} g(x)\right\} \hat{P}(x, t) \\
& \hat{V}(x):=V(x)+K(S \sin x+C \cos x) \\
& g(x):=\left[T+Q V^{\prime}(x)^{2}\right]^{1 / 2}
\end{aligned}
$$

Note that (9.13) represents a non-linear Fokker-Planck equation due to the implicit $\hat{P}(x, t)$-dependence of $\hat{V}(x)$ via (9.12) and (9.14). Especially, the linear superposition principle is not respected. This feature reflects the fact that while $P(x, t)$ in (9.11) is self-averaging with respect to the noises $f_{i}(t)$ and $y_{i}(t)$, it describes the particle density for a system with an arbitrary but fixed initial distribution of particles $P\left(x, t_{0}\right)$. A statistical ensemble average over different initial particle distributions is no longer captured by (9.13), in clear contrast to single-particle systems described e.g. by a linear master equation of the form (2.17), or more general, finite- $N$ particle systems. As usual in the context of phase transitions, the basic reason for this structural difference is the thermodynamic limit $N \rightarrow \infty$ in concert with the mean field coupling in (9.7), entailing the exact self-averaging property of the particle distribution (9.11) in this limit $N \rightarrow \infty$. The non-linear character of the Fokker-Planck equation opens the possibility that different initial conditions $P\left(x, t_{0}\right)$ display a different long time behavior, again in contrast to the typical asymptotic uniqueness (ergodicity) of linear Fokker-Planck equations [82, 83, 100, 108, 109]. The reason for this possibility of ergodicity breaking with all its consequences (spontaneous symmetry breaking, phase transitions, etc.) is that the thermodynamic limit $N \rightarrow \infty$ does not commute with the "ergodicity limit" $t \rightarrow \infty$. In conclusion, equations (9.11)-(9.15) display the typical structure of a mean field theory, with $S$ and $C$ in (9.12) playing the role of order parameters which have to be determined self-consistently with the mean field equation (9.13) for the particle density.

Next, we discuss the behavior of $\hat{P}(x, t)$ in (9.13) for asymptotically large coupling strengths $K$ in (9.7). To keep things simple, we further assume that multiples of $L$ are the only minima of $V(x)$. As a consequence, all particles in (9.7) are forced to occupy

\footnotetext{
${ }^{6}$ To be precise, this means that a convolution (average) $\int P(x, t) h(x) d x$ of the particle density with an arbitrary but fixed, smooth test function $h(x)$ that vanishes as $x \rightarrow \pm \infty$, gives the same result with probability 1 for $N \rightarrow \infty$, independent of the realization of the noises $\xi_{i}(t)$ and $f_{i}(t)$.

${ }^{7}$ For later convenience, the argument $t$ is suppressed in $S$ and $C$.
} 
practically the same position $\mu(t)$ modulo $L$ and hence $\hat{P}(x, t)$ takes the form

$$
\hat{P}(x, t)=\sum_{n=-\infty}^{\infty} \delta(x-\mu(t)+n L) .
$$

Introducing (9.16) into (9.13) and operating on both sides with $\int_{\mu(t)-L / 2}^{\mu(t)+L / 2} d x x \ldots$ the equation of motion for $\mu(t)$ takes the form of a simple relaxation dynamics

$$
\begin{aligned}
& \dot{\mu}(t)=-\bar{U}^{\prime}(\mu(t)) \\
& \bar{U}(x):=V(x)-Q V^{\prime}(x)^{2} / 2 .
\end{aligned}
$$

For small $Q$, the extrema of $\bar{U}(x)$ in (9.18) are identical to those of $V(x)$. So, for any initial condition $\mu\left(t_{0}\right) \in(-L / 2, L / 2)$, the center of mass $\mu(t)$ in $(9.17)$ moves for $t \rightarrow \infty$ towards the minimum $x=0$ of $V(x)$, and $\hat{P}(x, t)$ approaches a stationary, symmetric limit $\hat{P}^{s t}(x)=P^{s t}(-x)$. However, this stationary solution $\mu(t) \equiv 0$ of (9.17) looses stability and two new stable fixed points appear when $Q$ in (9.18) exceeds the critical value

$$
Q_{c}:=1 / V^{\prime \prime}(0)
$$

One thus recovers a so-called noise induced nonequilibrium phase transition [64, 65, 744 752 with a concomitant spontaneous symmetry breaking of $P^{s t}(x)$.

If the coupling strength $K$ is no longer assumed to be very large, one has to solve the non-linear Fokker-Planck equation (9.18) numerically until transients have died out and for a representative sample of different initial conditions. In this way, a stationary and - apart from the obvious degeneracy when the symmetry is spontaneously broken unique long time limit $\hat{P}^{s t}(x)$ is obtained. In the symmetric phase $\left(P^{s t}(-x)=P^{s t}(x)\right)$, the order parameter $S$ from (9.11) vanishes, while a spontaneously broken symmetry is generically monitored by a non-zero $S$-value, see figures 9.1. Moreover, for large $K$, the above analytical prediction is confirmed by the numerics, for moderate $K$, one recovers a re-entrant behavior as a function of the potential fluctuation strength $Q$, and for small $K$, a phase with broken symmetry ceases to exist [64, 745].

For an intuitive understanding of why the system-intrinsic symmetry can be spontaneously broken, we return to a one-particle dynamics of the form (4.17). By averaging over the noise, this equation takes the form $\langle\dot{x}\rangle=-\left\langle V^{\prime}(x(t)) / \eta\right\rangle+\langle g(x(t)) \xi(t)\rangle$. On the other hand, evaluating the particle current by means of the probability current (4.25) according to (2.19), one obtains $\langle\dot{x}\rangle=-\left\langle V^{\prime}(x(t)) / \eta\right\rangle+\left\langle g^{\prime}(x(t)) g(x(t))\right\rangle / 2$. Upon comparison of these two expressions one recovers that

$$
\langle g(x(t)) \xi(t)\rangle=\left\langle\frac{1}{4} \frac{d}{d x}[g(x(t))]^{2}\right\rangle .
$$

In other words, the white noise $\xi(t)$ induces a systematic drift into the direction of increasing effective local temperature $T_{\text {eff }}(x):=g^{2}(x)$ (see (9.15)). To get a rough heuristic picture of how this so-called Stratonovich drift term [99] comes about, we imagine a force-free, overdamped Brownian particle starting at $x(0)=0$ in the presence of a high temperature in the region $x>0$ and a low temperature for $x<0$. Though the particle spends on the average the same amount of time on either side of $x=0$, the 


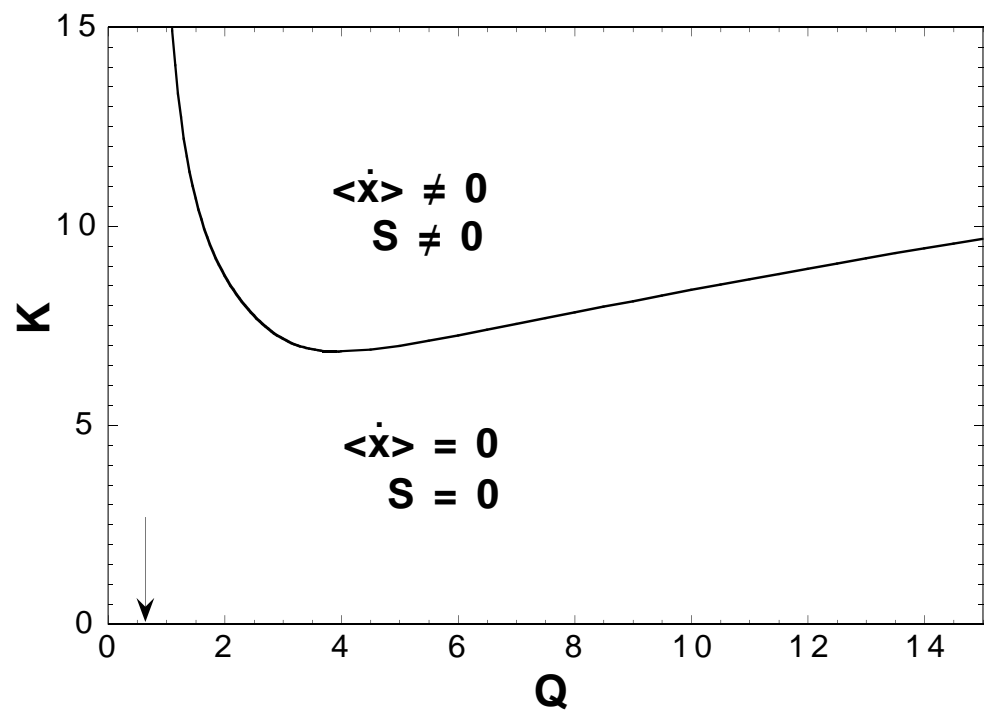

Figure 9.1: Phase diagram for the model (9.7)-(9.10) with $V(x)=-\cos x-0.15 \cos (2 x)$ (cf. equation (9.23) below) and $T=2$ in the thermodynamic limit $N \rightarrow \infty$ by numerically evolving the non-linear Fokker-Planck equation (9.13) until a steady state was reached. $\langle\dot{x}\rangle$ is the particle current, $S$ the order parameter from (9.12), and the arrow indicates the asymptotic phase boundary (9.19) for $K \rightarrow \infty$.

thermal random motion within $x>0$ is enhanced, leading to a net bias of the average particle position $\langle x(t)\rangle$ towards the right?

One can readily see by comparison with (9.15) that this noise induced drift term $(9.20)$ is indeed the origin of the second term on the right hand side of the effective potential (9.18), which governs the relaxation dynamics of the particle peak $\mu(t)$ in (9.17). Since the intensity of the multiplicative noise $f_{i}(t)$ in (9.7) has a minimum at the origin (modulo $L$ ), the noise induced drift pushes the particles away from this point $x=0$ and may lead, if the noise is strong enough and the particles cluster together sufficiently strongly, to a spontaneous dislocation of the peak of particles $\mu(t)$ towards one or the other side of the origin. If, on the other hand, the interaction is too weak in comparison to either the thermal or the potential fluctuations, then the random motions of the single particles are not sufficiently coordinated and a collective spontaneous symmetry breaking is therefore not expected. These heuristic arguments are confirmed by, and essentially explain the numerical phase diagram in figure 9.1 .

\subsubsection{Spontaneous ratchet effect}

We start by rewriting (9.7) in the form

$$
\dot{x}(t)=-V_{\mathrm{eff}}^{\prime}(x(t), f(t))+\xi(t),
$$

\footnotetext{
${ }^{8}$ Strictly speaking, the issue is rather subtle with respect to the correct order of the overdamped limit $m \rightarrow 0$ in (2.1), the white noise limit $\tau \rightarrow 0$ in (4.15), and the limit of a discontinuous temperature at $x=0$. Only if the limits are taken in the latter order $(m \rightarrow 0$ first, discontinuous temperature last), this explanation of the Stratonovich drift can be applied, see also (A.3) in Appendix A and the corresponding discussion in section 4.3.2.
} 
where we dropped the subscript $i$ and where

$$
V_{\text {eff }}(x, f(t)):=V(x)[1+f(t)]+K(C \cos x+S \sin x) .
$$

If there is no spontaneous symmetry breaking $\left(\hat{P}^{s t}(-x)=\hat{P}^{s t}(x)\right)$, then (9.12) implies $S=0$ and hence the pulsating potential (9.22) respects the symmetry condition (3.16) with $\Delta x=0$ due to (9.9). If, on the other hand, the symmetry of the system is spontaneously broken, then - in the generic case - we have that $S \neq 0$. Hence the symmetry condition (3.16) is generically violated and the occurrence of a ratchet effect with $\langle\dot{x}\rangle \neq 0$ is expected according to Curie's principle. There is, however, one prominent exception, namely a supersymmetric potential (9.29) excludes a current even if the symmetry of the system is spontaneously broken. For our present purposes it is sufficient to focus on the supersymmetry condition (3.41). Since the white noise $f(t)$ is time-inversion invariant, we see that for instance a pure cosine-potential $V(x)$ indeed leads to a supersymmetric effective potential in (9.22), whatever the values of $S$ and $C$ are. In order to break this supersymmetry, we can either modify the interaction in (9.7), or consider a colored noise $f_{i}(t)$, or, as we will do in the following, choose an augmented cosine potential of the form

$$
V(x)=-\cos x-A \cos (2 x)
$$

with $A \neq 0$.

Given that the potential (9.22) respects neither symmetry nor supersymmetry, each particle $(9.21)$ is expected to exhibit a ratchet effect $\langle\dot{x}\rangle \neq 0$ in the generic case [530,733, 736, as confirmed by the numerical result in 9 figure 9.1. The underlying mechanism is clearly of the general pulsating ratchet type, and according to 9.22) similar but not exactly identical to a fluctuating potential ratchet scheme from section 4.3 .

With the notation from (9.14), (9.15) we can rewrite (9.21) in yet another from, namely

$$
\begin{aligned}
& \dot{x}(t)=-\hat{V}^{\prime}(x(t))+\hat{\xi}(t) \\
& \langle\hat{\xi}(t) \hat{\xi}(s)\rangle=2 g(x(t)) \delta(t-s) .
\end{aligned}
$$

While $g(x)$ from (9.15) has its minima at the integer multiples of $L / 2$, the potential $\hat{V}(x)$ from (9.14) exhibits for $S \neq 0$ not only an asymmetric, ratchet-shaped profile, but also its extrema are generically shifted with respect to those of $g(x)$. From this viewpoint, the ratchet mechanism to which every single particle is subjected in the symmetry broken phase is thus of the Seebeck ratchet type from section 6.1.

Quantitatively, once the values of the order parameters $S$ and $C$ in the long time limit are known, the current follows readily along the lines of section 4.3 .2 with the

\footnotetext{
${ }^{9} \mathrm{~A}$ computer animation of this collective phenomenon is available on the internet under 753 . It is based on simulations of (9.7)-(9.10), (9.23) with $N=1000, T=2, Q=4, K=10, A=0.15$. Out of the 1000 particles, 100 are shown as green dots and one "tracer-particle" as a red dot. The position $x=-\pi$ is identified with $x=\pi$ (periodic boundary conditions). The initial particle distribution is symmetric about $x=0$. After a spontaneous breaking of the symmetry "to the right" $(S>0)$ an average particle current "to the left" $(\langle\dot{x}\rangle<0)$ can be observed.
} 
result

$$
\begin{aligned}
& \hat{P}^{s t}(x)=\mathcal{N} \frac{e^{-\phi(x)}}{g(x)} \int_{x}^{x+L} d y \frac{e^{\phi(y)}}{g(y)} \\
& \langle\dot{x}\rangle=L \mathcal{N}\left[1-e^{\phi(L)}\right] \\
& \phi(x):=\int_{0}^{x} d \bar{x} \hat{V}^{\prime}(\bar{x}) / g^{2}(\bar{x}),
\end{aligned}
$$

where the normalization $\mathcal{N}$ is fixed through (2.25). Thus, the current is finite unless $\phi(L)=0$, and its sign is given by that of $-\phi(L)$.

Specializing once again to large coupling strengths, we can exploit (9.16) to recast (9.28) into the simplified form

$$
\begin{aligned}
& \phi(L)=-\hat{K}_{1} \sin \mu \\
& \hat{K}_{n}:=\int_{-L / 2}^{L / 2} d x[K \cos x]^{n} / g(x)^{2},
\end{aligned}
$$

where $\mu:=\mu(t \rightarrow \infty)$ follows from (9.17). Here, a remarkable feature arises, entailing even more striking consequences later on. Namely, if $Q>Q_{c}$, and $\hat{K}_{1}<0$, which is the case whenever $A>0$ in (9.23), then the sign of $\mu(t)$ from (9.17) will, in the long time limit, be opposite to that of $\langle\dot{x}\rangle$. In other words, for a symmetry broken $P(x)$ with a peak to one side of $x=0$, the flux of particles will move just in the opposite direction! On average the particles surprisingly prefer to travel from their typical position, say $\mu(t \rightarrow \infty)<0$ down to the potential minimum of $V(x)$ at $x=0$ and then over the full barrier to their right rather than to directly surmount the partial remaining barrier that they typically see to their left.

\subsubsection{Negative mobility and anomalous hysteresis}

We now come to the response of the steady state current $\langle\dot{x}\rangle$ when an additional external force $F$ is added on the right hand side of (9.7). After making the replacement

$$
V(x) \mapsto V(x)-x F
$$

the entire analysis from sections 9.2.1 9.2 .3 can be repeated basically unchanged. For small $F$ in combination with $Q<Q_{c}$ and large $K$ we can then infer from (9.17), 9.19), (9.27), (9.29) after some calculations that

$$
\langle\dot{x}\rangle=F L \mathcal{N}\left(\hat{K}_{0}+\frac{\hat{K}_{1}}{\left[V^{\prime \prime}(0)\right]^{2}\left[Q_{c}-Q\right]}\right)+O\left(F^{3}\right) .
$$

Thus, for sufficiently large, negative $\hat{K}_{1}$, a negative zero-bias mobility (also called absolute negative mobility) is predicted $[530,733$. A numerical example for this remarkable behavior is shown in figure 9.2 (solid line). Apparently, the effect of pulling the particles to one side is analogous to that of a spontaneous symmetry breaking: it generates an effective, coupling-induced ratchet dynamics (9.21) in which the nonequilibrium fluctuations promote a current opposite to $F$. Upon approaching the phase boundary, the linear response of $P(x)$ to variations of $F$ diverges, hence the denominator $Q_{c}-Q$ in (9.32) and the very steep response curve in figure 9.2 .

\footnotetext{
${ }^{10}$ Note that such a current $\langle\dot{x}\rangle$ opposite to the applied force $F$ is not in contradiction with any kind of "stability criteria", cf. the discussion below (2.39).
} 


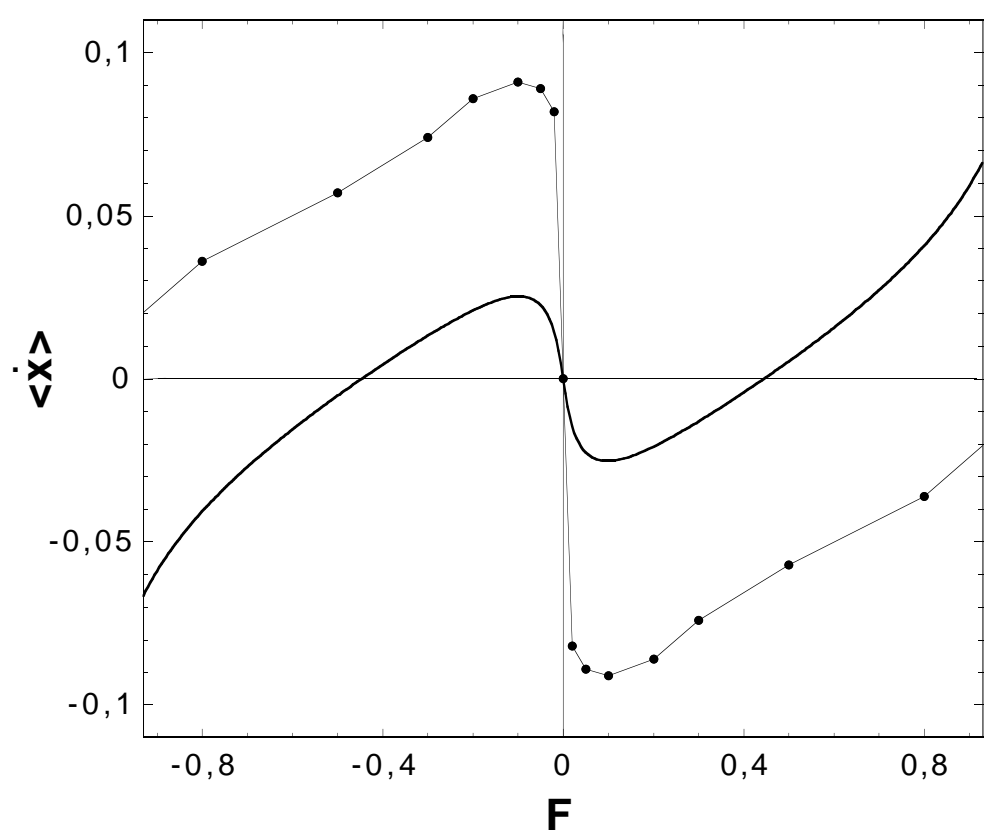

Figure 9.2: $\quad$ Solid line: Steady state current $\langle\dot{x}\rangle$ versus force $F$ for the model (9.7)(9.10), (9.23), 9.31) with $T=2, Q=2, K=8, A=0.15$ in the thermodynamic limit $N \rightarrow \infty$ by solving the non-linear Fokker-Planck equation (9.13). Interconnected dots: Simulations of (9.7) with nearest neighbor instead of global coupling (9.33) for a $64 * 64$ square lattice with periodic boundary conditions and modified parameters $Q=6, K=15$, averaged over 10 realizations.

We remark that for networks with dead-ends (see 422 and further references therein) and in the ratchet works [116, 423, 428, 429], a negative differential mobility (far away from $F=0$ ) has been reported, but not a current opposite to the applied force as in figure 9.2. Further, as illustrated by figure 2.5, in the current-versus-force characteristics for "standard", non-interacting ratchet models, a current opposite to the applied force is possible as well. However, as discussed in section 2.6.2, the ratchet effect is characterized by a current $\langle\dot{x}\rangle$ which is non-zero for $F=0$ and does not change its direction within an entire neighborhood of $F=0$. Accordingly, it inevitably involves some kind of symmetry breaking (for $F=0$ ), cf. section 3.2. In contrast, according to the characteristics of negative zero-bias mobility exemplified in figure 9.2. the current $\langle\dot{x}\rangle$ is always opposite to the (not too large) force $F$, independently of whether $F$ is positive or negative. Furthermore, the symmetry of the system (for $F=0$ ) is neither externally, nor intrinsically, nor spontaneously broken. In other words, the negative zero-bias mobility and the ratchet effect exhibit some striking similarities but also some fundamental differences. We also mention that so-called absolute negative conductance has been theoretically and experimentally studied in detail in the context of semiconductor devices [705, 739, 754 762], photovoltaic effects in ruby crystals [29, 763 765], tunnel junctions between superconductors with unequal energy gaps [766 768, and has been theoretically predicted for certain ionized gas mixtures 769 771. While these effects are in fact basically identical to negative zero-bias mobility, their origin is of a genuine quantum mechanical character which does not leave room for any kind of 
classical counterpart四.

For more general $F$ - and $Q$-values but still large $K$, the qualitative dependence of $\langle\dot{x}\rangle$ on $F$ follows from $(\overline{9.29})$ by observing how $\mu$ moves in the adiabatically changing potential $\bar{U}(x)$ from (9.18), (9.17). In this way, not only the continuation of the zerobias negative conductance beyond $F \simeq 0$ in figure 9.2 can be readily understood, but also its even more spectacular counterpart when $Q>Q_{c}$, namely an anomalous hysteresis-loop [530, 733, see figure 9.3. Its striking difference in comparison with a "normal" hysteresis-cycle, as observed e.g. in a ferromagnet or in the Jülicher-Prost model [557], is as follows: Given a spontaneous current in one or the other direction, we can apply a small additional force $F$ in the same direction, with the expected result of an increased current in that direction. But upon further increasing $F$, the current will, all of a sudden, switch its direction and run opposite to the applied force!

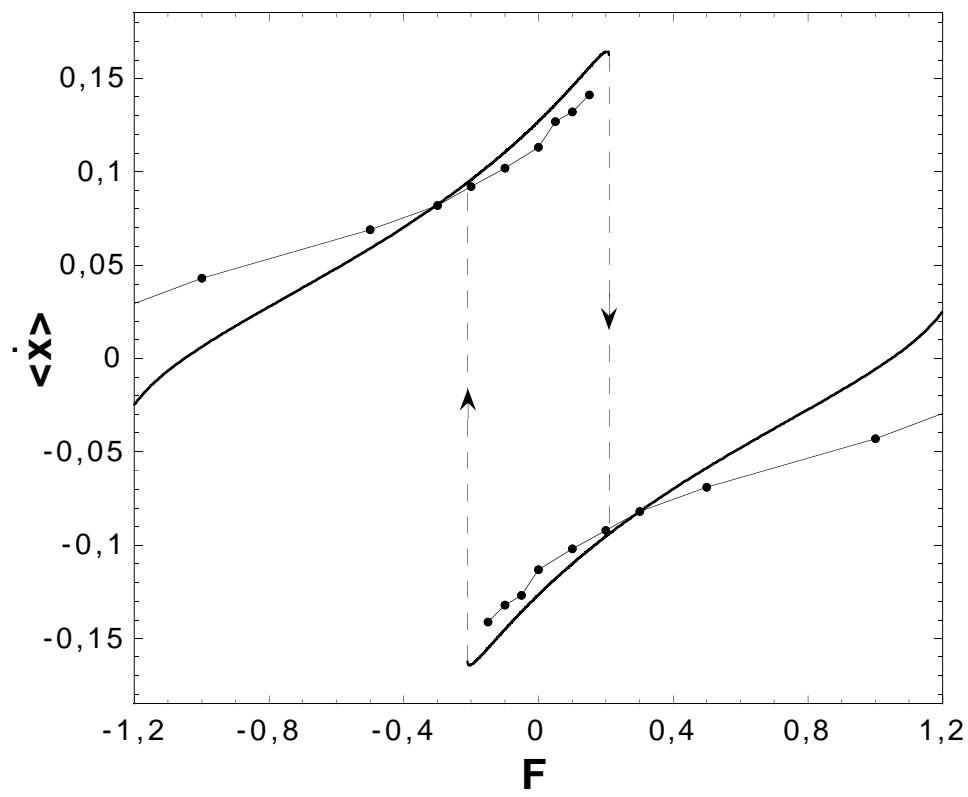

Figure 9.3: Same as in figure 9.2 but for $Q=4, K=10$ (global coupling) and $Q=10$, $K=20$ (nearest neighbor coupling).

In short, the anomalous response curves in figures 9.2 and 9.3 are basically the result of a competition between the effect of the bias $F$, favoring a current in that direction, and the ratchet-effect, which arises as a collective property and pumps particles in the opposite direction for $\hat{K}_{1}<0$. The coexistence of two solutions $\hat{P}^{s t}(x)$ over a certain $F$-interval when $Q>Q_{c}$ gives rise to the hysteresis, and the destabilization of one of them to the jumps of $\langle\dot{x}\rangle$ in figure 9.3 .

\subsubsection{Perspectives}

In this section we briefly discuss some generalizations and potential applications of our above considerations.

\footnotetext{
${ }^{11}$ Moreover, in the last three examples spatial periodicity is either not crucial or absent and in the case of tunnel junctions the spatial symmetry is intrinsically broken
} 
A first natural modification of the model (9.7) consist in replacing the global coupling in by a nearest neighbor coupling in $d$ dimensions, i.e.

$$
\frac{K}{N} \sum_{j=1}^{N} \sin \left(x_{j}(t)-x_{i}(t)\right) \mapsto \frac{K}{2 d} \sum_{<i j>} \sin \left(x_{j}(t)-x_{i}(t)\right),
$$

by associating the indices $i$ with the vertices of some $d$-dimensional lattice with periodic boundary conditions. As figures $9.2,9.3$ demonstrate, e.g. for a square lattice $(d=$ 2 ), the same qualitative phenomena as for global coupling are recovered, though the quantitative details are of course different.

Further generalizations 733] are: (i) The bare potential, represented by the " 1 " in the first term on the right hand side of (9.7) plays a very minor role; even without this term all results remain qualitatively unchanged. Similarly, the thermal noise strength $T$ is arbitrary, except that it must not vanish in the present model, but may even vanish in a somewhat modified setup [734. (ii) A strictly periodic interaction $K(x)$ is not necessary. For instance, one may add on top of the periodic a (not too strong) attractive interaction such as to keep the "cloud" of particles $x_{i}$ in (9.7) always well clustered.

Closely related studies on nonequilibrium phase transitions [772,773] suggest that also periodic instead of stochastic drivings $f_{i}(t)$ in (9.7) will lead to qualitatively similar results, see also [774]. Furthermore, a fluctuating force or rocking ratchet scheme instead of a fluctuating potential model, amounting in (9.7) to a substitution

$$
-V^{\prime}\left(x_{i}(t)\right)\left[1+f_{i}(t)\right] \mapsto-V^{\prime}\left(x_{i}(t)\right)+y_{i}(t),
$$

can apparently be employed as well [558]. Especially, the characteristic time scale of the driving $f_{i}(t)$ in (9.7) [735] and of $y_{i}(t)$ in (9.34) [558] may become asymptotically large.

It might appear [64, 745, 775] that taking the overdamped limit $m \rightarrow 0$ in (2.1) before the white noise limit $\tau \rightarrow 0$ of the Ornstein-Uhlenbeck noise $f_{i}(t)$ in (9.7), (9.10) (see also (4.15) and (4.16)) is an indispensable prerequisite for spontaneous symmetry breaking and spontaneous current, since only in this way 99, 294, a white noise $f_{i}(t)$ in the sense of Stratonovich and a concomitant noise induced drift term can arise. Our detailed analysis, however, reveals that the same phenomena can in fact still be encountered even if the white noise limit $\tau \rightarrow 0$ is performed prior to $m \rightarrow 0$, see also [776. In other words, finite inertia terms are also admissible on the left hand side of (9.7).

While a spontaneous ergodicity-breaking with all its above discussed consequences is clearly possible only in the thermodynamic limit $N \rightarrow \infty$, the same a priori restriction does not hold for the phenomenon of negative zero-bias mobility. Indeed, a stylized, spatially discretized descendant of the above discussed working model (9.7) with negative mobility for $N \geq 4$ has been presented in [737]. A different, experimentally realistic single particle system $(N=1)$ in two dimensions with negative mobility has been introduced in [777, while a game theoretic counterpart of the effect (cf. section 6.7) is due to 778.

In conclusion, the above revealed main phenomena seem to be rather robust against modifications and extensions of the considered model (9.7). Much like in equilibrium phase transitions, such an extremely simple model is thus expected to be of interest for a variety of different systems, corresponding to a "normal form" description that 
subsists after the irrelevant terms have been eliminated. Models of this type may be of relevance not only in the context of molecular motors (see section 7.4), but also for coupled phase oscillators [629, 728, 729], active rotator systems [779], charge density waves [780], and many other physical, chemical, and biological systems [627,781, 783]. For instance, one may also look at (9.7) as a planar XY-spin-model [784] exposed to a strong (but incoherent) electromagnetic irradiation [13, 28, 33, 286 289, 343], with the various effects of the photon-impacts (scattering, excitations of the host-crystal ions, etc.) roughly described by the non-equilibrium fluctuations $f_{i}(t)$. An experimental realization in a granular gas system is presently under construction. 


\section{Chapter 10}

\section{Conclusions}

The central theme of our review are transport phenomena in spatially periodic "Brownian motors" or "ratchet systems" induced by unbiased perturbations of the thermal equilibrium. Letting aside variations and extensions like diffusive transport, quenched spatial disorder, or questions of efficiency, our extensive discussions may be summarized under three main categories: i) Understanding and predicting the "ratchet effect" per $s e$, i.e. the occurrence (or not) of a directed average long-time current $\langle\dot{x}\rangle$. ii) Exploring qualitative features of the current as a function of various parameters, for example the sign of the current and the possible appearance of current inversions, monotonic versus non-monotonic "resonance-like" behavior with some type of "optimum", or the asymptotic behavior for fast, slow, and weak perturbations etc. iii) On the one hand, identifying particularly simple or counterintuitive "minimal models" and very general "normal forms" exhibiting a ratchet effect and/or current inversions. On the other hand, elaborating realistic models and their quantitative features with some specific experimental situation in mind.

For several of these questions, symmetry considerations play an important role. This is so basically due to Curies principle, stating that in the absence of prohibiting "systematic" symmetries, the appearance of a certain phenomenon (here: the ratchet effect) will be the rule, while its absence will be the exception. In our case, there are three such "systematic" symmetry conditions, each of which is sufficient to rule out the appearance of a ratchet effect: 1 . Detailed balance symmetry, implying that we are dealing with an equilibrium system and that a thermal equilibrium state will thus be approached in the long time limit. 2. (Spatial) symmetry as detailed in section 3.2. 3. Supersymmetry as detailed in section 3.5 in the overdamped limit and its counterpart (5.35) in the underdamped (deterministic Hamiltonian) limit. Closely related to these symmetry conditions, there are in addition a couple of "systematic" no go theorems for certain classes of ratchet systems, see at the beginning of section 4.3, at the end of section 4.4.2, and in section 6.4.1.

If all three above systematic symmetry conditions are violated, then a vanishing current is the exception, which may be termed an "accidental symmetry", and which

is usually connected with a current inversion. A very general method of tailoring such current inversions has been elaborated in section 3.6 together with a very simple and in fact obvious necessary and sufficient condition for their existence. Our ratchet classification scheme from section 3.3 is mainly based on the specific manner in which the second of the above systematic symmetries is broken. Depending on whether current inversions exist or not, we may speak of a "non trivial" and an "obvious" 
ratchet effect, exemplified by fluctuating potential and tilting ratchets and by (proper) traveling potential ratchets, respectively. In the first case, the direction of the current is obvious in some simple cases, but not at all in general, while in the second it is always rather clear.

We remark that for both, "systematic" and "accidental" symmetries, the result $\langle\dot{x}\rangle=0$ is unstable against completely general, generic variations of the model, while the property $\langle\dot{x}\rangle \neq 0$ is robust against such variations, i.e. "a finite current is the rule". The only difference is that for "systematic" symmetries, the hyperspace of parameters with $\langle\dot{x}\rangle=0$ (and thus the definition of the symmetry itself) can be easily expressed in terms of "natural" model parameters, while for "accidental" symmetries such a hyperspace exists as well but is very difficult to characterize. In this sense, there are actually no "accidental" symmetries, they are only very difficult to define and therefore "overlooked" within any "natural" invariance-considerations of the problem.

We note that the above symmetries 1.-3. refer strictly speaking to the (asymptotic) state and not to the system itself. Since the thermodynamic limit of infinitely many interacting subsystems may not commute with the long-time limit, and so an asymmetry of the initial condition may never disappear, some symmetry property of the system dynamics alone does not yet imply the corresponding asymptotic symmetry of the state (solution) in the case of extended systems. While this implication is still correct (leaving asside glass-like systems) for the first of the above mentioned symmetries (an equilibrium system implies an asymptotic equilibrium state), it may be incorrect in the second case of (spatial) symmetry: Even in a perfectly symmetric system, a spontaneous symmetry breaking of the asymptotic state may occur, leading to a spontaneous ratchet effect, see chapter 9 .

Regarding future perspectives of the field, the fact that many of the above symmetry considerations became clear only very recently suggest that further new theoretical results on a very basic conceptual level may still be to come. If a specific direction has to be named then the still rather fresh topic of coupled Brownian motors appears to be a particularly promising candidate, both theoretically (chapter 9) and with respect to biological applications (section 7.4). Further, there is a remarkable large and rapidly increasing number of exciting experimental studies, some of them with promising perspectives regarding technological applications. Whether Brownian motors offer just a new view or an entirely new paradigm with respect to the modeling of molecular motors (chapter 7 ) remains to be seen as well. 


\section{Acknowledgment}

The present review would not have been possible without the continuous support by P. Hänggi and his group and the very fruitful collaborations with C. Van den Broeck, R. Kawai, R. Bartussek, R. Häussler, M. Schreier, E. Pollak, C. Kettner, F. Müller, T.C. Elston, B. Lindner, L. Schimansky-Geier, I. Bena, M. Nagaoka, M. Grifoni, G.J. Schmid, and J. Lehmann. In preparing this work I have furthermore profited a lot from the scientific interaction with I. Goychuk, J. Luczka, P. Talkner, R.D. Astumian, M. Bier, J. Prost, F. Jülicher, C.R. Doering, P. Jung, G.-L. Ingold, H. Linke, D. Koelle, S. Weiss, M.I. Sokolov, T. Dittrich, R. Ketzmerick, H. Schanz, S. Flach, O. Yevtushenko, A. Lorke, C. Mennerat-Robilliard, C.M. Arizmendi, F. Sols, I. Zapata, T. Tél, M. Rubi, A. Perez-Madrid, M. Thorwart, R. Eichhorn, and J.M.R. Parrondo. Special thanks is due to P. Hänggi and I. Goychuk, for providing Refs. [1] and 675, respectively, to J.M.R. Parrondo and H. Linke for providing figures 2.1, 6.1 and figure 8.4, respectively, and to N. G. van Kampen for providing a copy of his thesis 6673]. Financial support by the DFG-Sachbeihilfe HA1517/13-2,13-4 and the Graduiertenkolleg GRK283 is gratefully acknowledged. 


\section{Appendix A}

\section{Supplementary material regarding section 2.1 .1}

We have modeled the two effects of the environment on the right hand side of equation (2.1) phenomenologically, and we will discuss in the next three subsections the rather far reaching implications of this specific phenomenological ansatz. Especially, we will argue that the assumptions of the environment being at thermal equilibrium and of a dissipation mechanism of the form $-\eta \dot{x}(t)$ competely fix the statistical properties of the additive fluctuations $\xi(t)$ in (2.1). While our line of reasoning will be conducted on a heuristic physical level, it still captures the essential ideas of mathematically more sophisticated and rigorous approaches [66, 77 97], see also sections 3.4.1, 6.4.3, and 8.1 .

\section{A.1 Gaussian white noise}

The fact that the friction force on the right hand side of (2.1) is linear in $\dot{x}(t)$, i.e. no spatial direction is preferred, suggests that - due to their common origin - also the thermal fluctuations are unbiased, that is (cf. (2.4))

$$
\langle\xi(t)\rangle=0
$$

for all times $t$, where $\langle\cdot\rangle$ indicates the average over independent realizations of the random process $\xi(t)$. Similarly, the fact that the friction force only depends on the present state of the system and not on what happened in the past has its counterpart in the assumption that the random fluctuations are uncorrelated in time, i.e.

$$
\langle\xi(t) \xi(s)\rangle=0 \text { if } t \neq s
$$

Furthermore, the fact that the friction involves no explicit time dependence has its correspondence in the time-translation invariance of all statistical properties of the fluctuations, i.e. the noise $\xi(t)$ is a stationary random process. Finally, the fact that the friction force acts permanently in time indicates that the same will be the case for the fluctuations. In other words, a noise $\xi(t)$ exhibiting rare but relatively strong "kicks", caused e.g. by impacts of single molecules in a diluted gas, is excluded. Technically speaking, one says that $\xi(t)$ cannot contain a shot noise component [178, 326 329]. During a small time interval, the effect of the environment thus consists of a large 
number of small and, according to (A.2) practically independent, contributions. Due to the central limit theorem円 the net effect of all these contributions on the particle $x(t)$ will thus be Gaussian distributed. Such a Gaussian random process which is unbiased (A.1) and uncorrelated in time (A.2) is called Gaussian white noise.

\section{A.2 Fluctuation-dissipation relation}

A crucial implicit assumption in (2.1) is the independence of the friction force, and hence also of the fluctuation force, from the system $x(t)$, i.e.2

$$
\langle\xi(t) x(s)\rangle=0
$$

for all times $t \geq s$. It reflects the assumption that the environment is given by a "huge" heat bath so that its properties are practically not influenced by the behavior of the "small" system $x(t)$. Especially, the statistical properties of the fluctuations will not depend on the choice of the potential $V(x)$ and we may set $V^{\prime}(x) \equiv 0$ in the following. One readily verifies that in this case the equation of motion (2.1) is solved by

$$
\dot{x}(t)=\dot{x}\left(t_{0}\right) e^{-\frac{\eta}{m}\left(t-t_{0}\right)}+\frac{1}{m} \int_{t_{0}}^{t} d t^{\prime} e^{-\frac{\eta}{m}\left(t-t^{\prime}\right)} \xi\left(t^{\prime}\right) .
$$

Choosing as initial time $t_{0}=-\infty$ it follows that

$$
\left\langle\dot{x}^{2}(t)\right\rangle=\frac{1}{m^{2}} \int_{-\infty}^{t} d t^{\prime} \int_{-\infty}^{t} d t^{\prime \prime} e^{-\frac{\eta}{m}\left(2 t-t^{\prime}-t^{\prime \prime}\right)}\left\langle\xi\left(t^{\prime}\right) \xi\left(t^{\prime \prime}\right)\right\rangle
$$

In view of (A.2), the integrand only contributes if $t^{\prime}=t^{\prime \prime}$ and the upper limit $t$ in the second integral can be furthermore extended to $+\infty$, i.e.

$$
\left\langle\dot{x}^{2}(t)\right\rangle=\frac{1}{m^{2}} \int_{-\infty}^{t} d t^{\prime} e^{-\frac{2 \eta}{m}\left(t-t^{\prime}\right)} \int_{-\infty}^{\infty} d t^{\prime \prime}\left\langle\xi\left(t^{\prime}\right) \xi\left(t^{\prime \prime}\right)\right\rangle
$$

Since the statistical properties of the fluctuations $\xi(t)$ are time-translation invariant, the second integral has the same value for all times $t^{\prime}$ and we can conclude that

$$
\int_{-\infty}^{\infty} d s\langle\xi(t) \xi(s)\rangle=2 \eta m\left\langle\dot{x}^{2}(t)\right\rangle
$$

for all times $t$. The left hand side of this equation is called the intensity of the noise $\xi(t)$ or the noise strength.

At this point, we make use of the fact that the environment is a heat bath at thermal equilibrium with temperature $T$. Since we have chosen as initial time $t_{0}=-\infty$, all

\footnotetext{
${ }^{1}$ In its simplest version - sufficient for our present purposes - the central limit theorem 100 states that if $r_{1}, \ldots, r_{N}$ are independent, identically distributed random variables with zero mean and unit variance then the sum $N^{-1 / 2}\left[r_{1}+\ldots+r_{N}\right]$ converges for $N \rightarrow \infty$ towards a Gaussian random variable of zero mean and unit variance.

${ }^{2}$ We remark that $m>0$ (cf. (2.1)) is understood in A.3). The properties (A.2) and (A.3) lead for $m \rightarrow 0$ to a Gaussian white noise $\xi(t)$ in the so-called Ito-sense 99.101 .

${ }^{3}$ The case $t<s$ is somewhat subtle and not needed in the following.
} 
transients have died out and the particle is in thermal equilibrium with the bath, satisfying the equipartition principle (for a one-dimensional dynamics)

$$
\frac{m}{2}\left\langle\dot{x}^{2}(t)\right\rangle=\frac{1}{2} k_{B} T,
$$

where $k_{B}$ is Boltzmann's constant. Collecting (A.2), A.7), A.8 we obtain the socalled fluctuation-dissipation relation 79 (cf. (2.5))

$$
\langle\xi(t) \xi(s)\rangle=2 \eta k_{B} T \delta(t-s),
$$

where $\delta(t)$ is Dirac's delta function. In other words, $\xi(t)$ is a Gaussian white noise of intensity $2 \eta k_{B} T$. Note that since $\xi(t)$ is a Gaussian random process, all its statistical properties are completely determined [99 101 already by the mean value (A.1) and the correlation (A.9).

\section{A.3 Einstein relation}

In the absence of the potential $V(x)$ in (2.1), we know that the particle exhibits a free thermal diffusion in one dimension with a diffusion constant $D$, i.e. for asymptotically large times $t$ we have that

$$
\left\langle x^{2}(t)\right\rangle=2 D t
$$

On the other hand, upon multiplying equation (2.1) by $x(t)$, averaging, and exploiting (A.3), we obtain

$$
m\langle\ddot{x}(t) x(t)\rangle=-\eta\langle\dot{x}(t) x(t)\rangle .
$$

The left hand side of this equation can be rewritten as

$$
m\langle\ddot{x}(t) x(t)\rangle=m \frac{d}{d t}\langle\dot{x}(t) x(t)\rangle-m\left\langle\dot{x}^{2}(t)\right\rangle .
$$

By differentiating (A.10) we have (for large $t$ ) that $\langle\dot{x}(t) x(t)\rangle=D$ and hence $d\langle\dot{x}(t) x(t)\rangle / d t=0$. Observing (A.8) we finally obtain from (A.11), (A.12) the socalled Einstein relation [77] (cf. (2.10))

$$
D=k_{B} T / \eta \text {. }
$$

Its most remarkable feature is that the diffusion in (A.10) does not depend on the mass $m$ of the particle $x(t)$ for asymptotically large times $t$.

\section{A.4 Dimensionless units and overdamped dynamics}

The objective of this section is to recast the stochastic dynamics (2.1), (A.9) into a dimensionless form, useful for qualitative theoretical considerations and indispensable for a numerical implementation.

\footnotetext{
${ }^{4}$ Corrections of order $o(t)$ are omitted in A.10 and we will tacitly assume that their time derivative approaches zero for $t \rightarrow \infty$ [67]. Furthermore, we note that this asymptotic result (A.10) is independent of the initial condition $x(0)$.
} 
We start with defining the barrier height

$$
\Delta V:=\max _{x}\{V(x)\}-\min _{x}\{V(x)\}
$$

between adjacent local minima of the periodic potential $V(x)$. Next, we introduce for the three dimensionful quantities $\eta, L$, and $\Delta V$ dimensionless counterparts $\hat{\eta}, \hat{L}$, and $\Delta \hat{V}$, which for the moment can still be freely chosen. With the definitions of the dimensionless quantities

$$
\begin{aligned}
\hat{t} & :=\alpha t, \quad \alpha:=\frac{\Delta V}{\eta L^{2}} \frac{\hat{\eta} \hat{L}^{2}}{\Delta \hat{V}} \\
\hat{x}(\hat{t}) & :=\frac{\hat{L}}{L} x(\hat{t} / \alpha) \\
\hat{V}(\hat{x}) & :=\frac{\Delta \hat{V}}{\Delta V} V(\hat{x} L / \hat{L})
\end{aligned}
$$

we can rewrite (2.1) in the dimensionless form

$$
\hat{m} \frac{d^{2} \hat{x}(\hat{t})}{d \hat{t}^{2}}+\hat{\eta} \frac{d \hat{x}(\hat{t})}{d \hat{t}}=-\frac{d \hat{V}(\hat{x}(\hat{t}))}{d \hat{x}}+\hat{\xi}(\hat{t})
$$

where $\hat{\xi}(\hat{t})$ is a dimensionless Gaussian white noise with correlation

$$
\langle\hat{\xi}(\hat{t}) \hat{\xi}(\hat{s})\rangle=2 \hat{\eta} \hat{k}_{B} \hat{T} \delta(\hat{t}-\hat{s}) .
$$

Furthermore, the dimensionless mass in $(\mathrm{A.18})$ is defined as

$$
\hat{m}:=m \frac{\Delta V}{\eta^{2} L^{2}} \frac{\hat{L}^{2} \hat{\eta}^{2}}{\Delta \hat{V}}
$$

and the dimensionless temperature in (A.19) as

$$
\hat{T}:=\frac{k_{B} T}{\Delta V} \frac{\Delta \hat{V}}{\hat{k}_{B}},
$$

where $\hat{k}_{B}$ may be chosen arbitrarily, e.g. $\hat{k}_{B}=1$.

Next we choose $\hat{\eta}, \hat{L}, \Delta \hat{V}$, and $\hat{k}_{B}$ all equal to unity. For the typically very small systems one has in mind, and for which thermal fluctuations play any notable role at all, the rescaled mass (A.20) then often turns out to be smaller than unity by many orders of magnitude, see e.g. in [291], while the dimensionless temperature (A.21) is of order unity or smaller . On the other hand, the period $\hat{L}$ and the barrier height $\Delta \hat{V}$ of the potential $\hat{V}(\hat{x})$ are both unity, so the derivative of this potential is typically of order unity as well. It is therefore quite plausible that in (A.18) the inertia term $\hat{m} d^{2} \hat{x}(\hat{t}) / d \hat{t}^{2}$ can be dropped in very good approximation. Admittedly, from a mathematical viewpoint, dropping the highest order derivative in a differential equation, especially in the presence of such an elusive object as the Gaussian white

\footnotetext{
${ }^{5}$ In the opposite case, i.e. if $\Delta V / k_{B} T$ is a small quantity (especially if $V(x)=$ const.) one has to replace $\Delta V$ by $k_{B} T$ in the definition (A.15) of $\alpha$, and similarly in (A.17), A.20). On condition that $m k_{B} T / \eta^{2} L^{2}$ is small, one can then drop the inertia term. The condition for arbitrary $\Delta V / k_{B} T$ is thus that the dimensionless quantity $m \eta^{-2} L^{-2} \max \left\{\Delta V, k_{B} T\right\}$ has to be a small quantity.
} 
noise $\hat{\xi}(\hat{t})$, may rise some concerns. A more careful treatment of this problem has been worked out e.g. in [294,469, 471, 472, 475, 488,489] with the same conclusion as along our simple heuristic argument. We finally note that letting $m \rightarrow 0$ affects neither the fluctuation-dissipation relation (A.9) nor Einstein's relation (A.13).

Finally, we turn to the the typical case that $\hat{m}$ is known to be a small quantity and we thus can set formally $m=0$ in (2.1). We thus recover the "minimal" SmoluchowskiFeynman ratchet model from (2.6). Introducing dimensionless units like before, one arrives again at (A.18) but now with $\hat{m}=0$ right from the beginning. In principle, $\hat{\eta}, \hat{L}$, and $\Delta \hat{V}$ may still be chosen arbitrarily. However, in most concrete cases it is convenient to assume that $\hat{L}$ and $\Delta \hat{V}$ are of order unity, but not necessary equal to 1 (e.g. $\hat{L}=2 \pi$ or $\hat{V}_{0}=1$ in (2.3) may sometimes be a more convenient choice), while $\hat{\eta}$ may still be a variable "control parameter" of the model. The implication of a dimensionless solution $\hat{x}(\hat{t})$ for the original, dimensionful system $x(t)$ is obvious. Especially, varying one parameter (e.g. $\hat{\eta}$ or $\hat{T}$ ) and keeping the others fixed, corresponds to exactly the same parameter-variation in the dimensionful system.

We finally remark that in the end one usually drops again the "hat"-symbols of the dimensionless quantities. Depending on the context, equation (2.6) may thus represent either the dimensionful or the dimensionless version of the model. 


\section{Appendix B}

\section{Alternative derivation of the Fokker-Planck equation}

In this appendix we give a derivation of the Fokker-Planck equation (2.14) by considering the corresponding overdamped stochastic dynamics (2.6) as limiting case of the discretized dynamics (2.7) when $\Delta t \rightarrow 0$.

To simplify notation, we use dimensionless units (see below equation (2.6)) with $k_{B}=\eta=1$. Next we recall that $\xi_{n}$ in (2.7) are independent, Gaussian distributed random variables with $\left\langle\xi_{n}\right\rangle=0$ and $\left\langle\xi_{n}^{2}\right\rangle=2 T / \Delta t$ (see (2.8)). It follows that for a particle (2.7), the conditional probability $P(x \mid y)$ to start out at time $t=t_{n}=n \Delta t$ from the point $x_{n}=y$ and to arrive one time step $\Delta t$ later at the point $x_{n+1}=x$ is Gaussian distributed about $x=y-\Delta t V^{\prime}(y)$ with variance $\left\langle\left(\Delta t \xi_{n}\right)^{2}\right\rangle=2 T \Delta t$, i.e.

$$
P(x \mid y)=(4 \pi T \Delta t)^{-1 / 2} \exp \left\{-\frac{\left[x-y+\Delta t V^{\prime}(y)\right]^{2}}{4 T \Delta t}\right\} .
$$

Furthermore, the probability distribution $P(x, t+\Delta t)$ at time $t+\Delta t$ is obviously related to that at time $t$ through the so-called Chapman-Kolmogorov equation [100]

$$
P(x, t+\Delta t)=\int_{-\infty}^{\infty} d y P(x \mid y) P(y, t) .
$$

After a change of the integration variable according to $z=(x-y) / \sqrt{\Delta t}$ we obtain

$$
\begin{aligned}
& P(x, t+\Delta t)= \\
& \int_{-\infty}^{\infty} \frac{d z}{(4 \pi T)^{1 / 2}} \exp \left\{-\frac{\left[z+\sqrt{\Delta t} V^{\prime}(x-\sqrt{\Delta t} z)\right]^{2}}{4 T}\right\} P(x-\sqrt{\Delta t} z, t) .
\end{aligned}
$$

Under the assumption that $P(x, t)$ behaves sufficiently well as $\Delta t \rightarrow 0$, we can expand the right hand side of (B.3) in powers of $\sqrt{\Delta t}$ and perform the remaining Gaussian integrals, with the result

$$
\begin{aligned}
& P(x, t+\Delta t)= \\
& P(x, t)+\Delta t \frac{\partial}{\partial x}\left\{V^{\prime}(x) P(x, t)\right\}+\Delta t T \frac{\partial^{2}}{\partial x^{2}} P(x, t)+o(\Delta t) .
\end{aligned}
$$

In particular, there is no contribution proportional to $\sqrt{\Delta t}$. In the limit $\Delta t \rightarrow 0$, the Fokker-Planck equation (2.14) now readily follows. 


\section{Appendix C}

\section{Perturbation analysis}

In this appendix we solve the Fokker-Planck equation (2.52) perturbatively for small time-periods $\mathcal{T}$ in (2.48) and zero load $F=0$. We recall that for evaluating the particle current $(2.53)$ we can focus on probability densities $\hat{P}(x, t)$ which are $L$-periodic in space and $\mathcal{T}$-periodic in time and that the function $\hat{T}(h)$ from (2.56) is assumed to be $\mathcal{T}$-independent. The latter assumption suggests to introduce

$$
W_{\mathcal{T}}(x, h):=\hat{P}(x, h \mathcal{T})
$$

so that the Fokker-Planck equation (2.52) takes the form

$$
\frac{\partial}{\partial h} W_{\mathcal{T}}(x, h)=\mathcal{T}\left\{\frac{\partial}{\partial x}\left[\frac{V^{\prime}(x)}{\eta} W_{\mathcal{T}}(x, h)\right]+\frac{k_{B} \hat{T}(h)}{\eta} \frac{\partial^{2}}{\partial x^{2}} W_{\mathcal{T}}(x, h)\right\} .
$$

The small quantity $\mathcal{T}$ on the right hand side of this equation furthermore suggest a power series ansatz

$$
W_{\mathcal{T}}(x, h)=\sum_{n=0}^{\infty} \mathcal{T}^{n} W_{n}(x, h)
$$

with $\mathcal{T}$-independent functions $W_{n}(x, h)$. From the periodicity and normalization of $\hat{P}(x, t)$ one readily finds that

$$
\begin{aligned}
& W_{n}(x+L, h)=W_{n}(x, h+1)=W_{n}(x, h) \\
& \int_{0}^{L} d x W_{n}(x, h)=\delta_{n, 0}
\end{aligned}
$$

for $n \geq 0$, where $\delta_{i, j}$ is the Kronecker delta.

Next the usual perturbation analysis argument is invoked: Introducing the ansatz (C.3) into the Fokker-Planck equation (C.2) and observing that this equation is supposed to hold for arbitrary $\mathcal{T}$ it follows that the coefficients of each power of $\mathcal{T}$ must be equal to zero separately. In the lowest order $\mathcal{T}^{0}$ it follows that

$$
\frac{\partial}{\partial h} W_{0}(x, h)=0
$$

i.e. $W_{0}(x, h)$ is equal to a $h$-independent but otherwise still unknown function $W_{0}(x)$. By introducing this function into (C.3), equating order $\mathcal{T}^{1}$-terms, and averaging over 
one time period $\mathcal{T}$ one obtains

$$
0=\frac{\partial}{\partial x}\left[\frac{V^{\prime}(x)}{\eta} W_{0}(x)\right]+\frac{k_{B} \bar{T}}{\eta} \frac{\partial^{2}}{\partial x^{2}} W_{0}(x),
$$

where the time-averaged temperature $\bar{T}$ is defined in (2.57). This ordinary second order equation for $W_{0}(x)$ can now be readily solved, with the two emerging integration constants being determined by the periodicity and normalization conditions (C.4), (C.5). The result is

$$
\begin{aligned}
& W_{0}(x)=Z^{-1} e^{-V(x) / k_{B} \bar{T}} \\
& Z:=\int_{0}^{L} d x e^{-V(x) / k_{B} \bar{T}}
\end{aligned}
$$

and the corresponding contribution of order $\mathcal{T}^{0}$ to the particle current $(2.53)$ is found to vanish. In other words, we have recovered in the limit $\mathcal{T} \rightarrow 0$ the same results as for a constant, time-averaged temperature $\bar{T}$ in section 2.4, in accordance with what one may have expected.

Proceeding in exactly the same way up to the next order $\mathcal{T}^{1}$ still gives a zero contribution to the particle current. It is only in the second order $\mathcal{T}^{2}$ that the first nontrivial contribution (2.58) is encountered. 


\section{Bibliography}

[1] M. v. Smoluchowski, Experimentell nachweisbare, der üblichen Thermodynamik widersprechende Molekularphänomene, Physik. Zeitschr. 13 (1912) 1069.

[2] R. P. Feynman, R. B. Leighton, M. Sands, The Feynman Lectures on Physics, Vol. 1, chapter 46, Addison Wesley, Reading MA, 1963.

[3] L. Brillouin, Can the rectifier become a thermodynamical demon?, Phys. Rev. 78 (1950) 627.

[4] A. F. Huxley, Muscle structure and theories of contraction, Prog. Biophys. 7 (1957) 255.

[5] S. M. Braxton, Synthesis and use of a novel class of ATP carbamates and a ratchet diffusion model for directed motion in muscle, $\mathrm{PhD}$ thesis, Washington State University, Pullman, WA, 1988.

[6] S. Braxton, R. G. Yount, A ratchet diffusion model for directed motion in muscle, Biophys. J. 55 (1989) 12a (abstr.).

[7] R. D. Vale, F. Oosawa, Protein motors and Maxwell's demons: Does mechanochemical transduction involve a thermal ratchet?, Adv. Biophys. 26 (1990) 97.

[8] S. Leibler, D. A. Huse, A physical model for motor proteins, C. R. Acad. Sci. Paris t. 313, Serie III (1991) 27.

[9] S. Leibler, D. A. Huse, Porters versus rowers: A unified stochastic model of motor proteins, J. Cell Biol. 121 (1993) 1357.

[10] N. J. Cordova, B. Ermentrout, G. F. Oster, Dynamics of single-motor molecules: The thermal ratchet model, Proc. Natl. Acad. Sci. USA 89 (1992) 339.

[11] M. O. Magnasco, Forced thermal ratchets, Phys. Rev. Lett. 71 (1993) 1477.

[12] M. O. Magnasco, Molecular combustion motors, Phys. Rev. Lett. 72 (1994) 2656.

[13] J. Prost, J.-F. Chauwin, L. Peliti, A. Ajdari, Asymmetric pumping of particles, Phys. Rev. Lett. 72 (1994) 2652.

[14] F. Jülicher, A. Ajdari, J. Prost, Modeling molecular motors, Rev. Mod. Phys. 69 (1997) 1269.

[15] R. D. Astumian, M. Bier, Fluctuation driven ratchets: molecular motors, Phys. Rev. Lett. 72 (1994) 1766. 
[16] R. D. Astumian, M. Bier, Mechanochemical coupling of the motion of molecular motors to ATP hydrolysis, Biophys. J. 70 (1996) 637.

[17] C. S. Peskin, G. B. Ermentrout, G. F. Oster, The correlation ratchet: a novel mechanism for generating directed motion by ATP hydrolysis, in: V. C. Mov, F. Guilak, R. Tran-Son-Tay, R. M. Hochmuth (Eds.), Cell Mechanics and Cellular Engineering, Springer, New York, 1994.

[18] C. S. Peskin, G. Oster, Coordinated hydrolysis explains the mechanical behavior of kinesin, Biophys. J. 68 (1995) 202s.

[19] E. H. Serpersu, T. Y. Tsong, Stimulation a Oubain-sensitive Rb ${ }^{+}$uptake in human erthrocytes with an external electric field, J. Membr. Biol. 74 (1983) 191.

[20] E. H. Serpersu, T. Y. Tsong, Activation of electrogenic $\mathrm{Rb}^{+}$transport of $(\mathrm{Na}, \mathrm{K})-$ ATPase by an electric field, J. Biol. Chem. 259 (1984) 7155.

[21] T. Y. Tsong, R. D. Astumian, Absorption and conversion of electric field energy by membrane bound ATPase, Bioelectrochem. Bioenerg. 15 (1986) 457.

[22] H. V. Westerhoff, T. Y. Tsong, P. B. Chock, Y. Chen, R. D. Astumian, How enzymes can capture and transmit free energy from an oscillating electric field, Proc. Natl. Acad. Sci. USA 83 (1986) 4734.

[23] W. Hoppe, W. Lohmann, H. Markl, H. Ziegler (Editors), Biophysics, Springer, Berlin, 1983.

[24] M. H. Friedman, Principles and models of biological Transport, Springer, Berlin, 1986.

[25] A. de Waele, W. H. Kraan, R. de Bruin Ouboter, K. W. Taconis, On the dc voltage across a double point contact between two superconductors at zero applied dc current in situations in which the junction is in the resistive region due to the circulating current of flux quantization, Physica (Utrecht) 37 (1967) 114.

[26] A. de Waele, R. de Bruin Ouboter, Quantum-interference phenomena in point contacts between two superconductors, Physica (Utrecht) 41 (1969) 225.

[27] A. M. Glas, D. van der Linde, T. J. Negran, High-voltage bulk photovoltaic effect and the photorefractive process in $\mathrm{LiNbO}_{3}$, Appl. Phys. Lett. 25 (1974) 233.

[28] V. I. Belinicher, B. I. Sturman, The photogalvanic effect in media lacking a center of symmetry, Sov. Phys. Usp. 23 (1980) 199, [Usp. Fiz. Nauk. 130 (1980) 415].

[29] B. I. Sturman, V. M. Fridkin, The photovoltaic and photorefractive effects in noncentrosymmetric materials, Gordon and Breach, Philadelphia, 1992.

[30] K. Seeger, W. Maurer, Nonlinear electronic transport in TTF-TCNQ observed by microwave harmonic mixing, Solid State Commun. 27 (1978) 603.

[31] W. Wonneberger, Stochastic theory of harmonic microwave mixing in periodic potentials, Solid State Commun. 30 (1979) 511. 
[32] A. L. R. Bug, B. J. Berne, Shaking-induced transition to a nonequilibrium state, Phys. Rev. Lett. 59 (1987) 948.

[33] M. Büttiker, Transport as a consequence of state-dependent diffusion, Z. Phys. B 68 (1987) 161.

[34] A. Ajdari, J. Prost, Mouvement induit par un potentiel periodique de basse symmetrie: dielectrophorese pulsee, C. R. Acad. Sci. Paris t. 315, Série II (1992) 1635.

[35] C. R. Doering, W. Horsthemke, J. Riordan, Nonequilibrium fluctuation-induced transport, Phys. Rev. Lett. 72 (1994) 2984.

[36] T. Hondou, Symmerty breaking by correlated noise in a multistable system, J. Phys. Soc. Jpn. 63 (1994) 2014.

[37] M. M. Millonas, M. I. Dykman, Transport and current reversal in stochastically driven ratchets, Phys. Lett. A 185 (1994) 65.

[38] J. Rousselet, L. Salome, A. Ajdari, J. Prost, Directional motion of Brownian particles induced by a periodic asymmetric potential, Nature 370 (1994) 446.

[39] A. Ajdari, D. Mukamel, L. Peliti, J. Prost, Rectified motion induced by ac forces in periodic structures, J. Phys. I France 4 (1994) 1551.

[40] J.-F. Chauwin, A. Ajdari, J. Prost, Force-free motion in asymmetric structures: a mechanism without diffusive steps, Europhys. Lett. 27 (1994) 421.

[41] A. Ajdari, Force-free motion in an asymmetric environment: a simple model for structured objects, J. Phys. I France 4 (1994) 1577.

[42] R. Bartussek, P. Hänggi, J. G. Kissner, Periodically rocked thermal ratchets, Europhys. Lett. 28 (1994) 459.

[43] J. Maddox, Making models of muscle contraction, Nature 365 (1993) 203.

[44] J. Maddox, More models of muscle contraction, Nature 368 (1994) 287.

[45] J. Maddox, Directed motion from random noise, Nature 369 (1994) 181.

[46] S. Leibler, Moving forward noisily, Nature 370 (1994) 412.

[47] C. Pöppe, Die ordnende Kraft der Asymmetrie, Spektrum der Wissenschaft, November issue (1994) 38.

[48] R. Bartussek, P. Hänggi, Brownsche Motoren, Phys. Bl. 51 (1995) 506.

[49] C. R. Doering, Randomly rattled ratchets, Il Nuovo Cimento D 17 (1995) 685.

[50] C. Ettl, Perpetuum mobile zweiter Art, Frankfurter Allgemeine Zeitung, 5. April (1995) 3.

[51] P. Hänggi, R. Bartussek, Brownian rectifiers: how to convert Brownian motion into directed transport, in: J. Parisi, S. C. Müller, W. Zimmermann (Eds.), Lecture notes in Physics Vol. 476: Nonlinear Physics of Complex Systems, Springer, Berlin, 1996. 
[52] K. Kostur, J. Luczka, Transport in ratchet-type systems, Acta Phys. Polon. B 27 (1996) 663.

[53] J. Luczka, Ratchets, molecular motors, and noise-induced transport, Cell. Mol. Biol. Lett. 1 (1996) 311.

[54] R. D. Astumian, Thermodynamics and kinetics of a Brownian motor, Science 276 (1997) 917.

[55] M. Bier, Brownian ratchets in physics and biology, Contemp. Phys. 38 (1997) 371.

[56] M. Bier, A motor protein model and how it relates to stochastic resonance, Feynman's ratchet, and Maxwell's demon, in: L. Schimansky-Geier, T. Pöschel (Eds.), Lecture Notes in Physics, Vol. 484, Springer, Berlin, 1997.

[57] R. D. Astumian and F. Moss (editors), Focus issue: The constructive role of noise in fluctuation driven transport and stochastic resonance, Chaos 8 (1998) 533-664.

[58] J. Luczka, Application of statistical mechanics to stochastic transport, Physica A 274 (1999) 200.

[59] R. D. Astumian, Ratchets, rectifiers, and demons: the constructive role of noise in free energy and signal transduction, in: J. Walleczek (Ed.), Self-organized biological dynamics and nonlinear control, Cambridge University Press, Cambridge, 2000 .

[60] C. Speicher, Die Kanalisierung des Zufalls, Neue Zürcher Zeitung, 9. Mai (2001) 49.

[61] R. D. Astumian, Making molecules into motors, Scientific American, July issue (2001) 56 .

[62] L. Gammaitoni, P. Hänggi, P. Jung, F. Marchesoni, Stochastic resonance, Rev. Mod. Phys. 70 (1998) 223.

[63] W. Horsthemke, R. Lefever, Noise-induced transitions, Springer, Berlin, 1984.

[64] C. Van den Broeck, J. M. R. Parrondo, R. Toral, R. Kawai, Nonequilibrium phase transitions induced by multiplicative noise, Phys. Rev. E 55 (1997) 4084.

[65] J. Garcia-Ojalvo, J. M. Sancho, Noise in spatially extended systems, Springer, New York, 1999.

[66] P. Hänggi, P. Talkner, M. Borkovec, Reaction rate theory: fifty years after Kramers, Rev. Mod. Phys. 62 (1990) 251.

[67] P. Hänggi, P. Jung, Colored noise in dynamical systems, Adv. Chem. Phys. 89 (1995) 239.

[68] P. Reimann, P. Hänggi, Surmounting fluctuating barriers: basic concepts and results, in: L. Schimansky-Geier, T. Pöschel (Eds.), Lecture Notes in Physics, Vol. 484, Springer, Berlin, 1997. 
[69] B. Schmittmann, R. K. P. Zia, Statistical mechanics of driven diffusive systems, in: C. Domb, J. L. Lebowitz (Eds.), Phase Transitions and Critical Phenomena, Vol. 17, Academic Press, London, 1995.

[70] G. M. Schütz, Exactly solvable models for many-body systems far from equilibrium, in: C. Domb, J. L. Lebowitz (Eds.), Phase Transitions and Critical Phenomena, Vol. 19, Academic Press, London, 2000.

[71] J. C. Maxwell, Theory of Heat, Longmans, Green and Co., London, 1872.

[72] H. S. Leff, A. F. Rex, Maxwell's demon, entropy, information, computing, Adam Hilger, Bristol, 1990.

[73] T. R. Kelly, I. Tellitu, J. P. Sestelo, In search of molecular ratchets, Angew. Chem. Int. Ed. Engl. 36 (1997) 1866.

[74] T. R. Kelly, J. P. Sestelo, I. Tellitu, New molecular devices: In search of a molecular ratchet, J. Org. Chem. 63 (1998) 3655.

[75] A. P. Davis, Tilting at windmills? The second law survives, Angew. Chem. Int. Ed. Engl. 37 (1998) 909.

[76] K. L. Sebastian, Molecular ratchets: verification of the principle of detailed balance and the second law of dynamics, Phys. Rev. E 61 (2000) 937.

[77] A. Einstein, Über die von der molekularkinetischen Theorie der Wärme geforderte Bewegung von in ruhenden Füssigkeiten suspendierten Teilchen, Ann. Phys. (Leipzig) 17 (1905) 549.

[78] A. Einstein, L. Hopf, Statistische Untersuchung der Bewegung eines Resonators in einem Strahlungsfeld, Ann. Phys. (Leipzig) 33 (1910) 1105.

[79] J. B. Johnson, Thermal agitation of electricity in conductors, Phys. Rev. 32 (1928) 97.

[80] H. Nyquist, Thermal agitation of electric charge in conductors, Phys. Rev. 32 (1928) 110.

[81] H. B. Callen, T. A. Welton, Irreversibility and generalized noise, Phys. Rev. 83 (1951) 34.

[82] P. G. Bergmann, J. L. Lebowitz, New approach to nonequilibrium processes, Phys. Rev. 99 (1955) 578.

[83] J. L. Lebowitz, P. G. Bergmann, Irreversible Gibbsian ensembles, Ann. Phys. (New York) 1 (1957) 1.

[84] V. B. Magalinskii, Dynamical model in the theory of the Brownian motion, Sov. Phys. JETP 9 (1959) 1381, [JETP 36 (1959) 1942].

[85] R. J. Rubin, Statistical dynamics of simple cubic lattices. Model for the study of Brownian motion, J. Math. Phys. 1 (1960) 309.

[86] J. L. Lebowitz, E. Rubin, Dynamical study of Brownian motion, Phys. Rev. 131 (1963) 2381. 
[87] P. Resibois, H. T. Davis, Transport equation of a Brownian particle in an external field, Physica (Utrecht) 30 (1964) 1077.

[88] P. Ullersma, An exactly solvable model for Brownian motion, Physica (Utrecht) 32 (1966) 27, 56, 74, and 90.

[89] R. Zwanzig, Nonlinear generalized Langevin equations, J. Stat. Phys. 9 (1973) 215.

[90] J. T. Hynes, J. M. Deutch, Nonequilibrium problems - projection operator techniques, in: D. Henerson (Ed.), Physical Chemistry, and advanced treatise, Academic Press, New York, 1975.

[91] H. Spohn, J. L. Lebowitz, Irreversible thermodynamics for quantum systems weakly coupled to thermal reservoirs, Adv. Chem. Phys. 38 (1978) 109.

[92] H. Grabert, P. Hänggi, P. Talkner, Microdynamics and nonlinear stochastic processes of gross variables, J. Stat. Phys. 22 (1980) 537.

[93] H. Grabert, Projection operator techniques in nonequilibrium statistical mechanics, Springer, Berlin, 1982.

[94] A. O. Caldeira, A. J. Leggett, Quantum tunneling in dissipative systems, Ann. Phys. (New York) 149 (1983) 374, erratum: Ann. Phys. (New York) 153 (1984) 445 .

[95] G. W. Ford, J. T. Lewis, R. F. O'Connell, Quantum Langevin equation, Phys. Rev. A 37 (1988) 4419.

[96] U. Weiss, Quantum dissipative systems, second enlarged Edition, World Scientific, Singapore, 1999.

[97] P. Reimann, A uniqueness-theorem for "linear" thermal baths, Chem. Phys. 268 (2001) 337.

[98] C. W. Gardiner, Handbook of stochastic methods for physics, chemistry, and the natural sciences, Springer, Berlin, 1983.

[99] H. Risken, The Fokker-Planck Equation, Springer, Berlin, 1984.

[100] N. G. van Kampen, Stochastic processes in physics and chemistry, revised and enlarged Edition, North-Holland, Amsterdam, 1992.

[101] P. Hänggi, H. Thomas, Stochastic processes: time evolution, symmetries, and linear response, Phys. Rep. 88 (1982) 207.

[102] H. A. Kramers, Brownian motion in a field of force and the diffusion model of chemical reactions, Physica (Utrecht) 8 (1940) 284.

[103] P. Hänggi, H. Grabert, P. Talkner, H. Thomas, Bistable systems: master equation versus Fokker-Planck modeling, Phys. Rev. A 29 (1984) 371.

[104] R. Zwanzig, Rate processes with dynamical disorder, Acc. Chem. Res. 23 (1990) 148. 
[105] G. Ryskin, Simple procedure for correcting equations of evolution: application to Markov processes, Phys. Rev. E 56 (1997) 5123.

[106] N. G. van Kampen, Die Fokker-Planck-Gleichung, Phys. Bl. 53 (1997) 1012.

[107] S. Chandrasekhar, Stochastic problems in physics and astronomy, Rev. Mod. Phys. 15 (1943) 1.

[108] P. T. Landsberg, Method of transition probabilities in quantum mechanics and quantum statistics, Phys. Rev. 96 (1954) 1420.

[109] F. Schlögl, Stochastic measures in nonequilibrium thermodynamics, Phys. Rep. $62(1980) 267$.

[110] J. M. R. Parrondo, P. Espanol, Criticism of Feynman's analysis of the ratchet as an engine, Am. J. Phys. 64 (1996) 1125.

[111] M. O. Magnasco, G. Stolovitzky, Feynman's ratchet and pawl, J. Stat. Phys. 93 (1998) 615.

[112] R. L. Stratonovich, Oscillator synchronization in the presence of noise, Radiotekhnika i elektronika 3 (1958) 497, english translation in Non-linear transformations of stochastic processes, edited by P. I. Kuznetsov, R. L Stratonovich, V. I. Tikhonov, Pergamon press, Oxford 1965.

[113] Y. M. Ivanchenko, L. A. Zil'berman, The Josephson effect for small tunnel contacts, Sov. Phys. JETP 28 (1969) 1272, [Zh. Eksp. Teor. Fiz 55 (1968) 2395].

[114] V. Ambegaokar, B. I. Halperin, Voltage due to thermal noise in the dc Josephson effect, Phys. Rev. Lett. 22 (1969) 1364.

[115] R. L. Stratonovich, Theory of Random Noise, Gordon and Breach, 1969.

[116] G. Cecchi, M. O. Magnasco, Negative resistance and rectification in Brownian transport, Phys. Rev. Lett. 76 (1996) 1968.

[117] P. Reimann, C. Van den Broeck, H. Linke, P. Hänggi, J. M. Rubi, A. PérezMadrid, Giant acceleration of free diffusion by use of tilted periodic potentials, Phys. Rev. Lett. 87 (2001) 010602.

[118] P. Reimann, R. Bartussek, R. Häussler, P. Hänggi, Brownian motors driven by temperature oscillations, Phys. Lett. A 215 (1996) 26.

[119] L. Ibarra-Bracamontes, V. Romero-Rochin, Stochastic ratchets with colored noise, Phys. Rev. E 56 (1997) 4048.

[120] C. R. Doering, Stochastic ratchets, Physica A 254 (1998) 1.

[121] S. M. Simon, C. S. Peskin, G. F. Oster, What drives the translocation of proteins, Proc. Natl. Acad. Sci. USA 89 (1992) 3770.

[122] C. S. Peskin, G. M. Odell, G. F. Oster, Cellular motions and thermal fluctuations: the Brownian ratchet, Biophys. J. 65 (1993) 316. 
[123] S. C. Kuo, J. L. McGrath, Steps and fluctuations of Listeria monocytogenes during actin-based motility, Nature 407 (2000) 1026.

[124] T. C. Elston, Models of post-translational protein translocation, Biophys. J. 79 (2000) 2235.

[125] W. Liebermeister, T. A. Rapoport, R. Heinrich, Ratcheting in post-translational protein translocation : a mathematical model, J. Mol. Biol. 305 (2001) 643.

[126] J. Luczka, T. Czernik, P. Hänggi, Symmetric white noise can induce directed current in ratchets, Phys. Rev. E 56 (1997) 3968.

[127] Y.-X. Li, Transport generated by fluctuating temperature, Physica A 238 (1997) 245.

[128] M. I. Sokolov, A. Blumen, Non-equilibrium directed diffusion and inherently irreversible heat engines, J. Phys. A 30 (1997) 3021.

[129] I. M. Sokolov, A. Blumen, Thermodynamical and mechanical efficiency of a ratchet pump, Chem. Phys. 235 (1998) 39.

[130] J.-D. Bao, Efficiency of energy transformation in an underdamped diffusion ratchet, Phys. Lett. A 267 (2000) 122.

[131] J. D. Bao, S. J. Liu, Broad-band colored noise: digital simulation and dynamical effect, Phys. Rev. E 60 (1999) 7572.

[132] K. S. Ralls, W. J. Skocpol, L. D. Jackel, R. E. Howard, L. A. Fetter, R. W. Epworth, D. M. Tennant, Discrete resistance switching in submicrometer silicon inversion layers: individual interface traps and low-frequency (1/f?) noise, Phys. Rev. Lett. 52 (1984) 228.

[133] C. J. Müller, J. M. van Ruitenbeek, L. J. de Jongh, Conductance and supercurrent discontinuities in atomic-scale metallic constrictions of variable width, Phys. Rev. Lett. 69 (1992) 140.

[134] B. Golding, N. M. Zimmerman, S. N. Coppersmith, Dissipative quantum tunneling of a single microscopic defect in a mesoscopic metal, Phys. Rev. Lett. 69 (1992) 998.

[135] D. C. Ralph, R. A. Buhrman, Observation of Kondo-scattering without magnetic impurities: a point contact study of two-level tunneling systems in metals, Phys. Rev. Lett. 69 (1992) 2118.

[136] R. J. Keijsers, O. I. Shklyarevskii, H. van Kempen, Point contact study of fast and slow two-level fluctuators in metallic glasses, Phys. Rev. Lett. 77 (1996) 3411.

[137] S. Kogan, Electronic noise and fluctuations in solids, Cambridge University Press, Cambridge, 1996.

[138] J. C. Smith, C. Berven, S. M. Goodnick, M. N. Wybourne, Nonequilibrium ransom telegraph switching in quantum point contacts, Physica B 227 (1996) 197. 
[139] J. Brini, P. Chenevier, P. d'Onofrino, P. Hruska, Higher order statistics of the thermal noise of ultrasmall MOSFET's, in: C. Claeys, E. Simeon (Eds.), Noise in Physical Systems and 1/f fluctuations, World Scientific, Singapore, 1997.

[140] A. W. Müller, Thermoelectric energy conversion could be an energy source of living organisms, Phys. Lett. A 96 (1983) 319.

[141] A. W. Müller, Were the first organisms heat engines? A new model for biogenesis and the early evolution of biological energy conversion, Prog. Biophys. Molec. Biol. 63 (1995) 193.

[142] A. J. Hunt, F. Gittes, J. Howard, The force exerted by a single kinesin molecule against a viscous load, Biophys. J. 67 (1994) 766.

[143] J. Howard, The movement of kinesin along microtubules, Annu. Rev. Physiol. 58 (1996) 703.

[144] T. Mitsui, H. Oshima, A self-induced translational model of myosin head motion in contracting muscle. I. Force-velocity relation and energy liberation, J. Musc. Res. Cell Motil. 9 (1988) 248.

[145] M. Bier, M. Kostur, Nonlinearly coupled chemical reactions, in: J. A. Freund, T. Pöschel (Eds.), Lecture Notes in Physics, Vol. 557, Springer, Berlin, 2000.

[146] M. Bier, M. Kostur, I. Derényi, R. D. Astumian, Nonlinearly coupled flows, Phys. Rev. E 61 (2000) 7184.

[147] P. Curie, Sur la symétrie dans les phénomenes physiques, symétrie d'un champ électrique et d'un champ magnétique, J. Phys. (Paris) 3. Série (théorique et appliqué) t. III (1894) 393.

[148] R. Graham, H. Haken, Generalized thermodynamic potential for Markoff systems in detailed balance and far from thermal equilibrium, Z. Phys. 243 (1971) 289.

[149] L. Onsager, Reciprocal relations in irreversible processes I, Phys. Rev. 37 (1931) 405.

[150] M. S. Green, Markoff random processes and the statistical mechanics of timedependent phenomena, J. Chem. Phys. 20 (1952) 1281.

[151] N. G. van Kampen, Derivation of the phenomenological equations from the master equation, Physica (Utrecht) 23 (1957) 707 and 816.

[152] R. Graham, H. Haken, Fluctuations and stability of stationary non-equilibrium systems in detailed balance, Z. Phys. 245 (1971) 141.

[153] N. G. van Kampen, Fluctuations in nonlinear systems, in: R. E. Burgess (Ed.), Fluctuation phenomena in solids, Academic Press, New York, 1965.

[154] R. McFee, Self-rectification in diodes and the second law of thermodynamics, Am. J. Phys. 39 (1971) 814.

[155] R. L. Stratonovich, Nonlinear nonequilibrium thermodynamics I, Springer, Berlin, 1992. 
[156] P. S. Landa, Noise-induced transport of Brownian particles with consideration for their mass, Phys. Rev. E 58 (1998) 1325.

[157] I. M. Sokolov, On the energetics of a nonlinear system rectifying thermal fluctuations, Europhys. Lett. 44 (1998) 278.

[158] R. D. Astumian, P. B. Chock, T. Y. Tsong, Y. Chen, H. V. Westerhoff, Can free energy be transduced from electric noise?, Proc. Natl. Acad. Sci. USA 84 (1987) 434 .

[159] W. T. H. Koch, R. Munser, W. Ruppel, P. Würfel, Bulk photovoltaic effect in $\mathrm{BaTiO}_{3}$, Solid State Commun. 17 (1975) 847.

[160] V. M. Asnin, A. A. Bakun, A. M. Danishevskii, E. L. Ivchenko, G. E. Pikus, A. A. Rogachev, "Circular" photogalvanic effect in optically active crystals, Solid State Commun. 30 (1979) 565.

[161] P. Reimann, P. Hänggi, Quantum features of Brownian motors and stochastic resonance, Chaos 8 (1998) 629.

[162] C. M. Arizmendi, F. Family, Approach to steady state current in ratchets, Physica A 232 (1996) 119.

[163] K. Handrich, F.-P. Ludwig, Friction coefficients and directed motion of asymmetric test particles, J. Stat. Phys. 86 (1997) 1067.

[164] A. Kolomeisky, B. Widom, A simplified "ratchet" model of molecular motors, J. Stat. Phys. 93 (1998) 633.

[165] O. Yevtushenkov, S. Flach, K. Richter, ac-driven phase-dependent directed current, Phys. Rev. E 61 (2000) 7215.

[166] I. Goychuk, P. Hänggi, Directed current without dissipation: re-incarnation of a Maxwell-Loschmidt-demon, in: J. A. Freund, T. Pöschel (Eds.), Lecture Notes in Physics, Vol. 557, Springer, Berlin, 2000.

[167] I. Goychuck, P. Hänggi, Minimal quantum Brownian rectifiers, J. Phys. Chem. 105 (2001) 6642.

[168] R. D. Cox, Renewal Theory, Methuen and Co., London, 1967.

[169] C. Van den Broeck, A glimpse into the world of random walks, in: J. L. MunozCobo, F. C. Difilippo (Eds.), Noise and nonlinear phenomena in nuclear systems, Plenum Publishing Corporation, 1989.

[170] P. Jung, J. G. Kissner, P. Hänggi, Regular and chaotic transport in asymmetric periodic potentials: inertia ratchets, Phys. Rev. Lett. 76 (1996) 3436.

[171] T. Harms, R. Lipowsky, Driven ratchets with disordered tracks, Phys. Rev. Lett. 79 (1997) 2895.

[172] J. A. Freund, L. Schimansky-Geier, Diffusion in discrete ratchets, Phys. Rev. E 60 (1999) 1304. 
[173] G. Constantini, F. Marchesoni, Threshold diffusion in a tilted washboard potential, Europhys. Lett. 48 (1999) 491.

[174] B. Lindner, M. Kostur, L. Schimansky-Geier, Optimal diffusive transport in a tilted periodic potential, Fluct. Noise Lett. 1 (2001) R25.

[175] C. Kettner, P. Reimann, P. Hänggi, F. Müller, Drift ratchet, Phys. Rev. E 61 (2000) 312.

[176] V. I. Klyatskin, Dynamic systems with parameter fluctuations of the telegraphicprocess type, Radiophys. Quantum Electron. 20 (1978) 382, [Radiofizika 20 (1977) 562].

[177] P. Hänggi, P. Riseborough, Activation rates in bistable systems in the presence of correlated noise, Phys. Rev. A 27 (1983) 3379.

[178] C. Van den Broeck, P. Hänggi, Activation rates for nonlinear stochastic flows driven by non-Gaussian noise, Phys. Rev. A 30 (1984) 2730.

[179] P. Hänggi, R. Bartussek, P. Talkner, J. Luczka, Noise-induced transport in symmetric periodic potentials: white shot noise versus deterministic noise, Europhys. Lett. 35 (1996) 315.

[180] D. R. Chialvo, M. I. Dykman, M. M. Millonas, Fluctuation-induced transport in a periodic potential: noise versus chaos, Phys. Rev. Lett. 78 (1997) 1605.

[181] E. Neumann, A. Pikovsky, Quasiperiodically driven Josephson junctions: strange nonchaotic attractors, symmetries, and transport, Submitted for publication.

[182] S. Weiss, D. Koelle, J. Müller, K. Barthel, R. Gross, Ratchet effect in dc SQUIDs, Europhys. Lett. 51 (2000) 499.

[183] S. Weiss, Ratscheneffekt in supraleitenden Quanteninterferenzdetektoren, PhD thesis (in German), Shaker Verlag, Aachen, 2000.

[184] S. Cilla, L. M. Floria, Mirror symmetry breaking through an internal degree of freedom leading to directional motion, Phys. Rev. E 63 (2001) 031110.

[185] W. H. Miller, Reaction-path dynamics for polyatomic systems, J. Chem. Phys. 87 (1983) 3811.

[186] D. Keller, C. Bustamante, The mechanochemistry of molecular motors, Biophys. J. 78 (2000) 541.

[187] R. D. Astumian, Adiabatic theory for fluctuation-induced transport on a periodic potential, J. Phys. Chem. 100 (1996) 19075.

[188] D. S. Liu, R. D. Astumian, T. Y. Tsong, Activation of the $\mathrm{Na}^{+}$and $\mathrm{Rb}^{+}$-pumping modes of (Na,K)-ATPase by an oscillating electric field, J. Biol. Chem. 265 (1990) 7260 .

[189] R. P. Feynman, F. L. Vernon, The theory of a general quantum systems interacting with a linear dissipative system, Ann. Phys. (New York) 24 (1963) 118. 
[190] M. M. Millonas, Self-consistent microscopic theory of fluctuation-induced transport, Phys. Rev. Lett. 74 (1995) 10, erratum: Phys. Rev. Lett. 75 (1995) 3027.

[191] A. M. Jayannavar, Simple model for Maxwell's-demon-type information engine, Phys. Rev. E 53 (1996) 2957.

[192] P. Hänggi, Generalized Langevin equations: a useful tool for the perplexed modeler of nonequilibrium fluctuations?, in: L. Schimansky-Geier, T. Pöschel (Eds.), Lecture Notes in Physics, Vol. 484, Springer, Berlin, 1997.

[193] I. Zapata, J. Luczka, F. Sols, P. Hänggi, Tunneling center as a source of voltage rectification in Josephson junctions, Phys. Rev. Lett. 80 (1998) 829.

[194] D. E. Postnov, A. P. Nikitin, V. S. Anishchenko, Control of the probability flux in a system of phase-controlled frequency self-tuning, Tech. Phys. Lett. 22 (1996) 352.

[195] A. P. Nikitin, D. E. Postnov, Effect of particle mass on the behavior of stochastic ratchets, Tech. Phys. Lett. 24 (1998) 61.

[196] M. Arrayas, R. Mannella, P. V. E. McClintock, A. J. McKane, N. D. Stein, Ratchet driven by quasimonochromatic noise, Phys. Rev. E 61 (2000) 139.

[197] L. Schimansky-Geier, M. Kschischo, T. Fricke, Flux of particles in sawtooth media, Phys. Rev. Lett. 79 (1997) 3335.

[198] J. M. R. Parrondo, Reversible ratchets as Brownian particles in an adiabatically changing periodic potential, Phys. Rev. E 57 (1998) 7297.

[199] J. M. R. Parrondo, J. M. Blanco, F. J. Cao, R. Brito, Efficiency of Brownian motors, Europhys. Lett. 43 (1998) 248.

[200] E. M. Höhberger, Magnetotransport in lateralen Halbleiterübergittern unter Einfluss von Symmetriebrechung, Diploma thesis (in German), Ludwig-MaximilianUniversität München (Germany), unpublished, 1999.

[201] J. M. R. Parrondo, B. Jimenez de Cisneros, R. Brito, Thermodynamics of isothermal Brownian motors, in: J. A. Freund, T. Pöschel (Eds.), Lecture Notes in Physics, Vol. 557, Springer, Berlin, 2000.

[202] E. M. Höhberger, A. Lorke, W. Wegscheider, M. Bichler, Adiabatic pumping of two-dimensional electrons in a ratchet-type lateral superlattice, Appl. Phys. Lett. 78 (2001) 2905.

[203] E. Witten, Dynamical breaking of supersymmetry, Nucl. Phys. B188 (1981) 513.

[204] R. Dutt, A. Khare, U. P. Sukhatme, Supersymmetry, shape invariance, and exactly solvable potentials, Am. J. Phys. 56 (1988) 163.

[205] C. M. Bender, F. Cooper, B. Freedman, A new strong-coupling expansion for quantum field theory based on the Langevin equation, Nucl. Phys. B219 (1983) 61. 
[206] M. Bernstein, L. S. Brown, Supersymmetry and the bistable Fokker-Planck equation, Phys. Rev. Lett. 52 (1984) 1933.

[207] F. Marchesoni, P. Sodano, M. Zanetti, Supersymmetry and bistable soft potentials, Phys. Rev. Lett. 61 (1988) 1143.

[208] G. Junker, Supersymmetric methods in quantum and statistical physics, Springer, Berlin, 1996.

[209] L. F. Favella, Brownian motions and quantum mechanics, Ann. Inst. Henri Poincaré 7 (1967) 77.

[210] H. Tomita, A. Ito, H. Kidachi, Eigenvalue problem of metastability in macrosystems, Prog. Theor. Phys. 56 (1976) 786.

[211] P. Jung, P. Hänggi, Amplification of small signals via stochastic resonance, Phys. Rev. A 44 (1991) 8032.

[212] T. Leibler, F. Marchesoni, H. Risken, Colored noise and bistable Fokker-Planck equations, Phys. Rev. Lett. 59 (1987) 1381, erratum: Phys. Rev. Lett. 60 (1988) 659.

[213] T. Leibler, F. Marchesoni, H. Risken, Numerical analysis of stochastic relaxation in bistable systems driven by colored noise, Phys. Rev. A 38 (1988) 983.

[214] R. Kanada, K. Sasaki, Thermal ratchets with symmetric potentials, J. Phys. Soc. Jpn. 68 (1999) 3759.

[215] O. Yevtushenko, S. Flach, Y. Zolotaryuk, A. A. Ovchinikov, Rectification of current in ac-driven nonlinear systems and symmetry properties of the Boltzmann equation, Europhys. Lett. 54 (2001) 141.

[216] B. Yan, R. M. Miura, Y.-D. Chen, Direction reversal of fluctuation-induced biased Brownian motion in distorted ratchets, J. Theor. Biol. 210 (2001) 141.

[217] P. Reimann, Supersymmetric ratchets, Phys. Rev. Lett. 86 (2001) 4992.

[218] H.-J. Breymayer, H. Risken, H. D. Vollmer, W. Wonneberger, Harmonic mixing in a cosine potential for large damping and arbitrary field strengths, Appl. Phys. B 28 (1982) 335 .

[219] W. Wonneberger, H.-J. Breymayer, Broadband current noise and ac induced current steps by a moving charge density wave domain, Z. Phys. B 56 (1984) 241.

[220] H.-J. Breymayer, Harmonic mixing in a cosine potential for arbitrary damping, Appl. Phys. A 33 (1984) 1.

[221] S. Flach, O. Yevtushenko, Y. Zolotaryuk, Directed current due to broken timespace symmetry, Phys. Rev. Lett. 84 (2000) 2358.

[222] B. Lindner, L. Schimansky-Geier, P. Reimann, P. Hänggi, Mass separation by ratchets, in: J. B. Kadtke, A. Bulsara (Eds.), Applied Nonlinear Dynamics and Stochastic Systems near the Millennium, AIP Proc. 411, 1997. 
[223] B. Lindner, L. Schimansky-Geier, P. Reimann, P. Hänggi, M. Nagaoka, Inertia ratchets: a numerical study versus theory, Phys. Rev. E 59 (1999) 1417.

[224] M. Bier, Reversal of noise induced flow, Phys. Lett. A 211 (1996) 12.

[225] I. Derényi, A. Ajdari, Collective transport of particles in a "flashing" periodic potential, Phys. Rev. E 54 (1996) R5.

[226] C. Berghaus, U. Kahlert, J. Schnakenberg, Current reversal induced by a cyclic stochastic process, Phys. Lett. A 224 (1997) 243.

[227] R. Bartussek, P. Hänggi, B. Lindner, L. Schimansky-Geier, Ratchets driven by harmonic and white noise, Physica D 109 (1997) 17.

[228] M. Schreier, P. Reimann, P. Hänggi, E. Pollak, Giant enhancement of diffusion and particle separation in rocked periodic potentials, Europhys. Lett. 44 (1998) 416.

[229] E. Abad, A. Mielke, Brownian motion in fluctuating periodic potentials, Ann. Phys. (Leipzig) 7 (1998) 9.

[230] J. L. Mateos, Chaotic transport and current reversal in deterministic ratchets, Phys. Rev. Lett. 84 (2000) 258.

[231] J. L. Mateos, Current reversals in chaotic ratchets, Acta Physica Polonica B 32 (2001) 307.

[232] M. Kostur, J. Luczka, Multiple current reversals in Brownian ratchets, Phys. Rev. E 63 (2001) 021101.

[233] B. Derrida, Y. Pomeau, Classical diffusion on a random chain, Phys. Rev. Lett. 48 (1982) 627.

[234] B. Derrida, Velocity and diffusion constants of a periodic one-dimensional hopping model, J. Stat. Phys. 31 (1983) 433.

[235] Z. Koza, General technique of calculating the drift velocity and diffusion coefficient in arbitrary periodic systems, J. Phys. A 32 (1999) 7637.

[236] K. W. Kehr, K. Mussawisade, T. Wichmann, W. Dieterich, Rectification by hopping motion through nonsymmetric potentials with strong bias, Phys. Rev. E 56 (1997) R2351.

[237] I. Derényi, C. Lee, A.-L. Barabasi, Ratchet effect in surface electromigration: smoothing surfaces by an ac field, Phys. Rev. Lett. 80 (1998) 1473.

[238] C. R. Doering, J. C. Gadoua, Resonant activation over a fluctuating barrier, Phys. Rev. Lett. 69 (1992) 2318.

[239] P. Hänggi, Dynamics of nonlinear oscillators with fluctuating parameters, Phys. Lett. A 78 (1980) 304.

[240] D. L. Stein, C. R. Doering, R. G. Palmer, J. L. van Hemmen, R. M. McLaughlin, Escape over fluctuating barrier: the white noise limit, J. Phys. A 23 (1990) L203. 
[241] U. Zürcher, C. R. Doering, Thermally activated escape over fluctuating barriers, Phys. Rev. E 47 (1993) 3862.

[242] M. Bier, R. D. Astumian, Matching a diffusive and a kinetic approach for escape over a fluctuating barrier, Phys. Rev. Lett. 71 (1993) 1649.

[243] P. Pechukas, P. Hänggi, Rates of activated processes with fluctuating barriers, Phys. Rev. Lett. 73 (1994) 2772.

[244] P. Hänggi, Escape over fluctuating barriers driven by colored noise, Chem. Phys. 180 (1994) 157.

[245] P. Reimann, Surmounting fluctuating barriers: A simple model in discrete time, Phys. Rev. E 49 (1994) 4938.

[246] P. Reimann, Thermally driven escape with fluctuating potentials: A new type of resonant activation, Phys. Rev. Lett. 74 (1995) 4576.

[247] P. Reimann, Thermally activated escape with potential fluctuations driven by an Ornstein-Uhlenbeck process, Phys. Rev. E 52 (1995) 1579.

[248] A. J. R. Madureira, P. Hänggi, V. Buonamano, W. A. Rodriguez, Escape from a fluctuating double well, Phys. Rev. E 51 (1995) 3849.

[249] R. Bartussek, A. J. R. Madureira, P. Hänggi, Surmounting a fluctuating double well: a numerical study, Phys. Rev. E 52 (1995) R2149.

[250] P. Reimann, T. C. Elston, Kramers rate for thermal plus dichotomous noise applied to ratchets, Phys. Rev. Lett. 77 (1996) 5328.

[251] J. Iwaniszewski, Escape over a fluctuating barrier: limits of small and large correlation times, Phys. Rev. E 54 (1996) 3173.

[252] P. Reimann, R. Bartussek, P. Hänggi, Reaction rates when barriers fluctuate: a singular perturbation approach, Chem. Phys. 235 (1998) 11.

[253] P. Reimann, G. J. Schmid, P. Hänggi, Universal equivalence of mean-first passage time and Kramers rate, Phys. Rev. E 60 (1999) R1.

[254] J. Ankerhold, P. Pechukas, Mathematical aspects of the fluctuating barrier problem. Explicit equilibrium and relaxation solutions, Physica A 261 (1999) 458.

[255] Y. Chen, Asymmetry and external noise-induced free energy transduction, Proc. Natl. Acad. Sci. USA 84 (1987) 729.

[256] T. D. Xie, P. Marszalek, Y. Chen, T. Y. Tsong, Recognition and processing of randomly fluctuating electric signals by Na,K-ATPase, Biophys. J. 67 (1994) 1247.

[257] T. D. Xie, Y. Chen, P. Marszalek, T. Y. Tsong, Fluctuation-driven directional flow in biochemical cycles: further study of electric activation of Na,K pumps, Biophys. J. 72 (1997) 2496.

[258] P. Jung, Periodically driven stochastic systems, Phys. Rep. 234 (1993) 175. 
[259] M. I. Dykman, H. Rabitz, V. N. Smelyanskiy, B. E. Vugmeister, Resonant directed diffusion in nonadiabatically driven systems, Phys. Rev. Lett. 79 (1997) 1178 .

[260] V. N. Smelyanskiy, M. I. Dykman, B. Golding, Time oscillations of escape rates in periodically driven systems, Phys. Rev. Lett. 82 (1999) 3193.

[261] P. Talkner, Stochastic resonance in the semiadiabatic limit, New J. Phys. 1 (1999) 4.

[262] R. Graham, T. Tél, On the weak-noise limit of Fokker-Planck models, J. Stat. Phys. 35 (1984) 729.

[263] J. Lehmann, P. Reimann, P. Hänggi, Surmounting oscillating barriers, Phys. Rev. Lett. 84 (2000) 1639.

[264] J. Lehmann, P. Reimann, P. Hänggi, Surmounting oscillating barriers: Pathintegral approach for weak noise, Phys. Rev. E 62 (2000) 6282.

[265] R. Bartussek, P. Reimann, P. Hänggi, Precise numerics versus theory for correlation ratchets, Phys. Rev. Lett. 76 (1996) 1166.

[266] A. Mielke, Transport in a fluctuating potential, Ann. Phys. (Leipzig) 4 (1995) 721.

[267] J. D. Bao, Y. Abe, Y. Z. Zhuo, Competition and cooperation between thermal noise and external driving force, Physica A 277 (2000) 127.

[268] J. Plata, Rocked thermal ratchets: the high frequency limit, Phys. Rev. E 57 (1998) 5154.

[269] G. N. Milstein, M. V. Tretyakov, Mean velocity of noise-induced transport in the limit of weak periodic forcing, J. Phys. A 32 (1999) 5795.

[270] http://monet.physik.unibas.ch/ elmer/bm.

[271] J.-F. Chauwin, A. Ajdari, J. Prost, Current reversal in asymmetric pumping, Europhys. Lett. 32 (1995) 373, erratum: Europhys. Lett. 32 (1995) 699.

[272] Y. Chen, B. Yan, R. Miura, Asymmetry and direction reversal in fluctuationinduced biased Brownian motion, Phys. Rev. E 60 (1999) 3771.

[273] L. P. Faucheux, A. Libchaber, Selection of Brownian particles, J. Chem. Soc. Faraday Trans. 91 (1995) 3163.

[274] T. Schnelle, T. Müller, G. Gradl, S. G. Shirley, G. Fuhr, Dielectrophoretic manipulation of suspended submicron particles, Electrophoresis 21 (2000) 66.

[275] L. P. Faucheux, L. S. Bourdieu, P. D. Kaplan, A. Libchaber, Optical thermal ratchet, Phys. Rev. Lett. 74 (1995) 1504.

[276] L. Gorre-Talini, S. Jeanjean, P. Silberzan, Sorting of Brownian particles by pulsed application of an asymmetric potential, Phys. Rev. E 56 (1997) 2025. 
[277] L. Gorre-Talini, J. P. Spatz, P. Silberzan, Dielectrophoretic ratchets, Chaos 8 (1998) 650.

[278] J. S. Bader, R. W. Hammond, S. A. Henck, M. W. Deem, G. A. McDermott, J. M. Bustillo, J. W. Simpson, G. T. Mulhern, J. M. Rothberg, DNA transport by a micromachined Brownian ratchet device, Proc. Natl. Acad. Sci. USA 96 (1999) 13165.

[279] R. W. Hammond, J. S. Bader, S. A. Henck, M. W. Deem, G. A. McDermott, J. M. Bustillo, J. M. Rothberg, Differential transport of DNA by a rectified Brownian motion device, Electrophoresis 21 (2000) 74.

[280] L. Rowen, G. Mahairas, L. Hood, Sequencing the human genome, Science 278 (1997) 605 .

[281] E. Lai, B. W. Birren (Eds.), Electrophoresis of large DNA molecules, Cold Spring Harbor Laboratory Press, Cold Spring Harbor, NY, 1990.

[282] G. Führ, U. Zimmermann, S. Shirley, Cell motion in time varying fields: principles and potential, in: U. Zimmermann, S. Neil (Eds.), Electromanipulation of Cells, CRC Press, Boca Raton, 1996, p. 259.

[283] D. Ertas, Lateral separation of macromolecules and polyelectrolytes in microlithographic arrays, Phys. Rev. Lett. 80 (1998) 1548.

[284] M. B. Tarlie, R. D. Astumian, Optimal modulation of a Browinan ratchet and enhanced sensitivity to a weak external force, Proc. Natl. Acad. Sci. USA 95 (1998) 2039.

[285] A. Ajdari, Pumping liquids using asymmetric electrode arrays, Phys. Rev. E 61 (2000) R45.

[286] I. Janossy, Molecular interpretation of the absorbtion-induced optical reorientation of nematic liquid crystals, Phys. Rev. E 49 (1994) 2957.

[287] P. Palffy-Muhoray, E. Weinan, Orientational ratchets and angular momentum balance in the Janossy effect, Mol. Cryst. Liq. Cryst. 320 (1998) 193.

[288] T. Kosa, E. Weinan, P. Palffy-Muhoray, Brownian motors in the photoalignment of liquid crystals, Int. J. Eng. Sci. 38 (2000) 1077.

[289] M. Kreuzer, L. Marrucci, D. Paparo, Light-induced modification of kinetic molecular properties: enhancement of optical Kerr effect in absorbing liquids, photoinduced torque and molecular motors in dye-doped nematics, J. Nonlin. Opt. Phys. Mat. 9 (2000) 157.

[290] E. Goldobin, A. Sterck, D. Koelle, Josephson vortex in a ratchet potential: Theory, Phys. Rev. E 63 (2001) 031111.

[291] J. Kula, M. Kostur, J. Luczka, Brownian motion controlled by dichotomic and thermal fluctuations, Chem. Phys. 235 (1998) 27. 
[292] M. Bier, R. D. Astumian, Biasing Brownian motion in different directions in a 3-state fluctuating potential and an application for the separation of small particles, Phys. Rev. Lett. 76 (1996) 4277.

[293] P. Reimann, Current reversal in a white noise driven flashing ratchet, Phys. Rep. 290 (1997) 149.

[294] R. Graham, A. Schenzle, Stabilization by multiplicative noise, Phys. Rev. A 26 (1982) 1676.

[295] J.-D. Bao, Y.-Z. Zhuo, X.-Z. Wu, Diffusion current for a system in a periodic potential driven by additive colored noise, Phys. Lett. A 215 (1996) 154.

[296] J.-D. Bao, Y.-Z. Zhuo, X.-Z. Wu, Effect of multiplicative noise on fluctuationinduced transport, Phys. Lett. A 217 (1996) 241.

[297] K. Lee, W. Sung, Effects of nonequilibrium fluctuations on ionic transport through biomembranes, Phys. Rev. E 60 (1999) 4681.

[298] Archimedes of Syracuse, Ca. 250 b. c., unpublished.

[299] M. Borromeo, F. Marchesoni, Brownian surfers, Phys. Lett. A 249 (1998) 8457.

[300] K. M. Jansons, G. D. Lythe, Stochastic Stokes drift, Phys. Rev. Lett. 81 (1998) 3136 .

[301] C. Van den Broeck, Stokes' drift: an exact result, Europhys. Lett. 46 (1999) 1.

[302] M. Borromeo, F. Marchesoni, Thermal conveyers, Appl. Phys. Lett. 75 (1999) 1024.

[303] Y.-X. Li, X.-Z. Wu, Y.-Z. Zhuo, Brownian motors: solitary waves and efficiency, Physica A 286 (2000) 147.

[304] I. Bena, M. Copelli, C. Van den Broeck, Stokes' drift: a rocking ratchet, J. Stat. Phys. 101 (2000) 415.

[305] G. G. Stokes, On the theory of oscillatory waves, Trans. Camb. Philos. Soc. 8 (1847) 441.

[306] O. N. Mesquita, S. Kane, J. P. Gollub, Transport by capillary waves: fluctuating Stokes drift, Phys. Rev. A 45 (1992) 3700.

[307] D. J. Thouless, Quantization of transport, Phys. Rev. B 27 (1983) 6083.

[308] M. Switkes, C. M. Marcus, K. Campman, A. C. Gossard, An adiabatic electron pump, Science 283 (1999) 1905.

[309] M. Wagner, F. Sols, Subsea electron transport: pumping deep within the Fermi sea, Phys. Rev. Lett. 83 (1999) 4377.

[310] F. Sols, M. Wagner, Pipeline model of a Fermi-sea electron pump, Ann. Phys. (Leipzig) 9 (2000) 776. 
[311] R. D. Astumian, I. Derényi, Towards a chemically driven molecular electron pump, Phys. Rev. Lett. 86 (2001) 3859.

[312] L. P. Kouwenhoven, A. T. Johnson, N. C. van der Vaart, C. P. M. Harmans, Quantized current in a quantum-dot turnstile using oscillating tunnel barriers, Phys. Rev. Lett. 67 (1991) 1626.

[313] L. P. Kouwenhoven, A. T. Johnson, N. C. van der Vaart, A. van der Enden, C. P. M. Harmans, C. T. Foxton, Quantized current in a quantum dot turnstile, Z. Phys. B 85 (1991) 381.

[314] H. Pothier, P. Lafarge, C. Urbina, D. Esteve, M. H. Devoret, Single-electron pump based on charging effects, Europhys. Lett. 17 (1992) 249.

[315] M. W. Keller, J. M. Martinis, N. M. Zimmerman, A. H. Steinbach, Accuracy of electron counting using a 7-junction electron pump, Appl. Phys. Lett. 69 (1996) 1804 .

[316] J. Weis, R. J. Haug, K. von Klitzing, K. Poog, Single-electron tunneling transistor as a current rectifier with potential-controlled current polarity, Semicond. Sci. Technol. 10 (1995) 877.

[317] X. Wang, T. Junno, S.-B. Carlsson, C. Thelander, L. Samuelson, Coulomb blockade ratchet, cond-mat/9910444.

[318] R. Landauer, M. Büttiker, Drift and diffusion in reversible computation, Physica Scripta T9 (1985) 155.

[319] L. P. Faucheux, G. Stolovitzky, A. Libchaber, Periodic forcing of a Brownian particle, Phys. Rev. E 51 (1995) 5239.

[320] V. I. Talyanskii, J. M. Shilton, M. Pepper, C. G. Smith, C. J. B. Ford, E. H. Linfield, D. A. Ritchie, G. A. C. Jones, Single electron transport in a one-dimensional channel by high frequency surface acoustic waves, Phys. Rev. B 56 (1997) 15180.

[321] C. Rocke, S. Zimmermann, A. Wixforth, J. P. Kotthaus, G. Böhm, G. Weinmann, Acoustically driven storage of light in a quantum well, Phys. Rev. Lett. 78 (1997) 4099 .

[322] D. E. Postnov, A. P. Nikitin, V. S. Anishchenko, Synchronization of the mean velocity of a particle in stochastic ratchets with a running wave, Phys. Rev. E 58 (1998) 1662.

[323] A. N. Malakhov, A new model of Brownian transport, Izv. VUZ "AND" 6 (1998) 105.

[324] S. Sasa, T. Shibata, Brownian motors driven by particle exchange, J. Phys. Soc. Jpn. 67 (1998) 1918.

[325] K. Fukui, J. H. Frederick, J. I. Cline, Chiral dissociation dynamics of molecular ratchets: Preferential sense of rotatory motion in microscopic systems, Phys. Rev. E 58 (1998) 929. 
[326] S. O. Rice, in: N. Wax (Ed.), Selected papers on noise and stochastic processes, Dover, New York, 1954.

[327] P. Hänggi, Correlation functions and master equations of generalized (nonMarkovian) Langevin equations, Z. Phys. B 31 (1978) 407.

[328] P. Hänggi, Langevin description of Markovian integro-differential master equations, Z. Phys. B 36 (1980) 271.

[329] C. Van den Broeck, On the relation between white shot noise, Gaussian white noise and the dichotomic Markov process, J. Stat. Phys. 31 (1983) 467.

[330] J. Luczka, R. Bartussek, P. Hänggi, White-noise-induced transport in periodic structures, Europhys. Lett. 31 (1995) 431.

[331] T. Czernik, J. Kula, J. Luczka, P. Hänggi, Thermal ratchets driven by Poissonian white shot noise, Phys. Rev. E 55 (1997) 4057.

[332] T. Czernik, J. Luczka, Rectified steady flow induced by white shot noise: diffusive and non-diffusive regimes, Ann. Phys. (Leipzig) 9 (2000) 721.

[333] T. Czernik, M. Niemiec, J. Luczka, Brownian motors driven by Poissonian fluctuations, Acta Physica Polonica B 32 (2001) 321.

[334] Y.-X. Li, Y.-Z. Zhuo, Directed motion induced by shifting ratchet, Int. J. Mod. Phys. B 14 (2000) 2609.

[335] Y. Chen, Asymmetric cycling and biased movement of Brownian particles in fluctuating symmetric potentials, Phys. Rev. Lett. 79 (1997) 3117.

[336] Y.-X. Li, Directed motion induced by a cyclic stochastic process, Mod. Phys. Lett. B 11 (1997) 713.

[337] L. Gorre-Talini, P. Silberzan, Force-free motion of a mercury drop alternatively submitted to shifted asymmetric potentials, J. Phys. I France 7 (1997) 1475.

[338] M. Porto, M. Urbakh, J. Klafter, Molecular motor that never steps backwards, Phys. Rev. Lett. 85 (2000) 491.

[339] C. Mennerat-Robilliard, D. Lucas, S. Guibal, J. Tabosa, C. Jurczak, J.-Y. Courtois, G. Grynberg, Ratchet for cold Rubidium atoms: The asymmetric optical lattice, Phys. Rev. Lett. 82 (1999) 851.

[340] T. R. Kelly, H. De Silva, R. A. Silva, Unidirectional rotary motion in a molecular system, Nature 401 (1999) 150.

[341] A. P. Davis, Synthetic molecular motors, Nature 401 (1999) 120.

[342] T. R. Kelly, Progress towards rationally designed molecular motors, Acc. Chem. Res. 34 (2001) 514.

[343] N. Koumura, R. W. J. Zijistra, R. A. van Delden, N. Harada, B. L. Feringa, Light-driven monodirectional molecular motor, Nature 401 (1999) 152. 
[344] J. K. Gimzewski, C. Joachim, R. R. Schlittler, V. Langlais, H. Tang, I. Johannsen, Rotation of a single molecule within a supramolecular bearing, Science 281 (1998) 531.

[345] J. K. Gimzewski, C. Joachim, Nanoscale science of single molecules using local probes, Science 283 (1999) 1683.

[346] B. Alberts, D. Bray, J. Lewis, M. Raff, K. Roberts, J. D. Watson, The molecular biology of the cell, Garland, New York, 1994.

[347] R. D. Astumian, P. B. Chock, T. Tsong, H. V. Westerhoff, Effects of oscillations and energy-driven fluctuations on the dynamics of enzyme catalysis and freeenergy transduction, Phys. Rev. A 39 (1989) 6416.

[348] R. D. Astumian, B. Robertson, Nonlinear effect of an oscillating electric field on membrane proteins, J. Chem. Phys. 91 (1989) 4891.

[349] A. Fulinski, Noise-stimulated active transport in biological cell membranes, Phys. Lett. A 193 (1994) 267.

[350] A. Fulinski, Active transport in biological membranes and stochastic resonance, Phys. Rev. Lett. 79 (1997) 4926.

[351] A. Fulinski, Barrier fluctuations and stochastic resonance in membrane transport, Chaos 8 (1998) 549.

[352] R. D. Astumian, I. Derényi, Fluctuation driven transport and models of molecular motors and pumps, Eur. Biophys. J. 27 (1998) 474.

[353] T. Y. Tsong, Cellular transduction of periodic and stochastic signals by electroconformational coupling, in: J. Walleczek (Ed.), Self-organized biological dynamics and nonlinear control, Cambridge University Press, Cambridge, 2000.

[354] B. Robertson, R. D. Astumian, Michaelis-Menten equation for an enzyme in an oscillating electric field, Biophys. J. 58 (1990) 969.

[355] A. Mielke, Noise induced transport, Ann. Phys. (Leipzig) 4 (1995) 476.

[356] D. R. Chialvo, M. M. Millonas, Asymmetric unbiased fluctuations are sufficient for the operation of a correlation ratchet, Phys. Lett. A 209 (1995) 26.

[357] I. Zapata, R. Bartussek, F. Sols, P. Hänggi, Voltage rectification by a SQUID ratchet, Phys. Rev. Lett. 77 (1996) 2292.

[358] M. M. Millonas, D. R. Chialvo, Nonequilibrium fluctuation-induced phase transport in Josephson junctions, Phys. Rev. E 53 (1996) 2239.

[359] A. Sarmiento, H. Larralde, Deterministic transport in ratchets, Phys. Rev. E 59 (1999) 4878.

[360] P. S. Landa, P. V. E. McClintock, Changes in the dynamical behavior of nonlinear systems induced by noise, Phys. Rep. 323 (1999) 1.

[361] T. C. Elston, C. R. Doering, Numerical and analytical studys of nonequilibrium fluctuation-induced transport processes, J. Stat. Phys. 83 (1996) 359. 
[362] W. Forst, Theory of unimolecular reactions, Academic Press, New York, 1973.

[363] C. R. Doering, L. A. Dontcheva, M. M. Klosek, Constructive role of noise: fast fluctuation asymptotics of transport in stochastic ratchets, Chaos 8 (1998) 643.

[364] H. Kohler, A. Mielke, Noise-induced transport at zero temperature, J. Phys. A 31 (1998) 1929.

[365] R. Mankin, A. Ainsaar, Current reversals in ratchets driven by trichotomous noise, Phys. Rev. E 61 (2000) 6359.

[366] M. M. Klosek, R. W. Cox, Steady-state currents in sharp stochastic ratchets, Phys. Rev E 60 (1999) 3727.

[367] J. Kula, T. Czernik, J. Luczka, Transport generated by dichotomic fluctuations, Phys. Lett. A 214 (1996) 14.

[368] J. Kula, T. Czernik, J. Luczka, Brownian ratchets: transport controlled by thermal noise, Phys. Rev. Lett. 80 (1998) 1377.

[369] C. M. Arizmendi, F. Family, Memory correlation effect on thermal ratchets, Physica A 251 (1998) 368.

[370] R. Bartussek, Stochastische Ratschen, PhD thesis (in German), Logos Verlag, Berlin, 1998.

[371] T. E. Dialynas, K. Lindenberg, G. P. Tsironis, Ratchet motion induced by deterministic and correlated stochastic forces, Phys. Rev. E 56 (2000) 3976.

[372] J. D. Bao, Rectification of different colored noise, Phys. Lett. A 256 (1999) 356.

[373] E. Cortes, Ratchet motion induced by a correlated stochastic force, Physica A 275 (2000) 78.

[374] R. Bartussek, Ratchets driven by colored Gaussian noise, in: L. SchimanskyGeier, T. Pöschel (Eds.), Lecture Notes in Physics, Vol. 484, Springer, Berlin, 1997.

[375] F. Marchesoni, Conceptional design of a molecular shuttle, Phys. Lett. A 237 (1998) 126.

[376] P. Lancon, G. Batrouni, L. Lobry, N. Ostrowsky, Drift without flux: Brownian walker with a space-dependent diffusion coefficient, Europhys. Lett. 54 (2001) 28.

[377] R. von Baltz, W. Krauth, Theory of the bulk photovoltaic effect in pure crystals, Phys. Rev. B 23 (1981) 5590.

[378] L. I. Magarill, Photogalvanic effect in asymmetric lateral superlattice, Physica E 9 (2001) 625.

[379] V. I. Fal'ko, D. E. Khmel'nitskii, Mesoscopic photovoltaic effect in microjunctions, Sov. Phys. JETP 68 (1989) 186, [Zh. Eksp. Teor. Fiz. 95 (1989) 328]. 
[380] J. Liu, M. A. Pennington, N. Giordano, Mesoscopic photovoltaic effect, Phys. Rev. B 45 (1992) 1267.

[381] G. Dalba, Y. Soldo, F. Rocca, V. M. Fridkin, P. Sainctavit, Giant bulk photovoltaic effect under linearly polarized x-ray synchrotron radiation, Phys. Rev. Lett. 74 (1995) 988.

[382] V. E. Kravtsov, V. I. Yudson, Directed current in mesoscopic rings induced by high-frequency electromagnetic field, Phys. Rev. Lett. 70 (1993) 210.

[383] A. G. Aronov, V. E. Kravtsov, Nonlinear properties of disordered normal-metal rings with magnetic flux, Phys. Rev. B 47 (1993) 13409.

[384] G. M. Shmelev, N. H. Song, G. I. Tsurkan, Photostimulated even acoustoelectric effect, Sov. Phys. J. (USA) 28 (1985) 161.

[385] M. V. Entin, Theory of the coherent photogalvanic effect, Sov. Phys. Semicond. $23(1989) 664$.

[386] R. Atanasov, A. Haché, J. L. P. Hughes, H. M. van Driel, J. E. Sipe, Coherent control of photocurrent generation in bulk semiconductors, Phys. Rev. Lett. 76 (1996) 1703.

[387] A. Haché, Y. Kostoulas, R. Atanasov, J. L. P. Hughes, J. E. Sipe, H. M. van Driel, Observation of controlled photocurrent in unbiased bulk GaAs, Phys. Rev. Lett. 78 (1997) 306.

[388] K. N. Alekseev, M. V. Erementchouk, F. V. Kusmartsev, Direct-current generation due to wave mixing in semiconductors, Europhys. Lett. 47 (1999) 595.

[389] P. Reimann, Rocking ratchets at high frequencies, in: J. A. Freund, T. Pöschel (Eds.), Lecture Notes in Physics, Vol. 557, Springer, Berlin, 2000.

[390] S. Shapiro, Josephson currents in superconducting tunneling: the effect of microwaves and other observations, Phys. Rev. Lett. 11 (1963) 80.

[391] P. Jung, P. Hänggi, Effect of periodic driving on the escape in periodic potentials, Ber. Bunsenges. Phys. Chem. 95 (1991) 311.

[392] L. Gorre, E. Ioannidis, P. Silberzan, Rectified motion of a mercury drop in an asymmetric structure, Europhys. Lett. 33 (1996) 267.

[393] F. Falo, P. J. Martinez, J. J. Mazo, S. Cilla, Ratchet potential for fluxons in Josephson-junction arrays, Europhys. Lett. 45 (1999) 700.

[394] E. Trias, J. J. Mazo, F. Falo, T. P. Orlando, Depinning of kinks in a Josephsonjunction ratchet array, Phys. Rev. E 61 (2000) 2257.

[395] G. Carapella, Relativistic flux quantum in a field-induced deterministic ratchet, Phys. Rev. B 63 (2001) 054515.

[396] C.-S. Lee, B. Janko, I. Derényi, A.-L. Barabasi, Reducing vortex density in superconductors using the "ratchet effect", Nature 400 (1999) 337. 
[397] J. F. Wambaugh, C. Reichhardt, C. J. Olson, F. Marchesoni, F. Nori, Superconducting fluxon pumps and lenses, Phys. Rev. Lett. 83 (1999) 5106.

[398] P. J. de Pablo, J. Colchero, J. Gomez-Herrero, A. Asenjo, M. Luna, P. A. Serena, A. M. Baro, Ratchet effect in surface electromigration detected with scanning force microscopy in gold micro-stripes, Surf. Sci. 464 (2000) 123.

[399] M. Barbi, M. Salerno, Phase locking effect and current reversals in deterministic underdamped ratchets, Phys. Rev. E 62 (2000) 1988.

[400] M. Barbi, M. Salerno, Stabilization of ratchet dynamics by weak periodic signals, Phys. Rev. E 63 (2001) 066212.

[401] C. M. Arizmendi, F. Family, A. L. Salas-Brito, Quenched disorder effects on deterministic inertia ratchets, Phys. Rev. E 63 (2001) 061104.

[402] H. Fujisaka, S. Grossmann, Chaos-induced diffusion in nonlinear discrete dynamics, Z. Phys. B 48 (1982) 261.

[403] T. Geisel, J. Nierwetberg, Onset of diffusion and universal scaling in chaotic systems, Phys. Rev. Lett. 48 (1982) 7.

[404] M. Schell, S. Fraser, R. Kapral, Diffusive dynamics in systems with translational symmetry: a one-dimensional-map model, Phys. Rev. A 26 (1982) 504.

[405] T. Geisel, J. Nierwetberg, Statistical properties of intermittent diffusion in chaotic systems, Z. Phys. B 56 (1984) 59.

[406] P. Reimann, Suppression of deterministic diffusion by noise, Phys. Rev. E 50 (1994) 727.

[407] P. Reimann, C. Van den Broeck, Intermittent diffusion in the presence of noise, Physica D 75 (1994) 509.

[408] R. Klages, J. R. Dorfman, Simple maps with fractal diffusion coefficient, Phys. Rev. Lett. 74 (1995) 387.

[409] O. Farago, Y. Kantor, Directed chaotic motion in a periodic potential, Physica A 249 (1998) 151.

[410] J. D. Meiss, Symplectic maps, variational principles, and transport, Rev. Mod. Phys. 64 (1992) 795.

[411] G. M. Zaslavsky, Chaotic dynamics and the origin of statistical laws, Phys. Today, August issue (1999) 39.

[412] S. Kovalyov, Phase space structure and anomalous diffusion in a rotational fluid experiment, Chaos 10 (2000) 153.

[413] T. Dittrich, R. Kretzmerick, M.-F. Otto, H. Schanz, Classical and quantum transport in deterministic Hamiltonian ratchets, Ann. Phys. (Leipzig) 9 (2000) 755 .

[414] H. Schanz, M.-F. Otto, R. Ketzmerick, T. Dittrich, Classical and quantum Hamiltonian ratchets, Phys. Rev. Lett. 87 (2001) 070601. 
[415] S. Denisov, S. Flach, Dynamical mechanism of dc current generation in driven Hamiltonian systems, Submitted for publication.

[416] J.-D. Bao, Y.-Z. Zhuo, Langevin simulation approach to a two-dimensional coupled flashing ratchet, Phys. Lett. A 239 (1998) 228.

[417] A. W. Ghosh, S. V. Khare, Rotation in an asymmetric multidiomensional periodic potential due to colored noise, Phys. Rev. Lett. 84 (2000) 5243.

[418] H. Qian, Vector field formalism and analysis for a class of thermal ratchets, Phys. Rev. Lett. 81 (1998) 3063.

[419] M. Kostur, L. Schimansky-Geier, Numerical study of diffusion induced transport in 2d systems, Phys. Lett. A 265 (2000) 337.

[420] P. Hänggi, P. Reimann, Quantum ratchet reroute electrons, Physics World 12 (1999) 21.

[421] M. Brooks, Quantum clockwork, New Scientist 2222 (2000) 29.

[422] V. Balakrishnan, C. Van den Broeck, Transport properties on a random comb, Physica A 217 (1995) 1.

[423] G. W. Slater, H. L. Guo, G. I. Nixon, Bidirectional transport of polyelectrolytes using self-modulating entropic ratchets, Phys. Rev. Lett. 78 (1997) 1170.

[424] C. Turmel, E. Brassard, R. Forsyth, K. Hood, G. W. Slater, J. Noorlandi, High resolution zero intergated field electrophoresis of DNA, in: E. Lai, B. W. Birren (Eds.), Electrophoresis of large DNA molecules, Cold Spring Harbor Laboratory Press, Cold Spring Harbor, NY, 1990.

[425] C. Desruisseaux, G. W. Slater, T. B. Kist, Trapping electrophoresis and ratchets: a theoretical study for DNA-protein complexes, Biophys. J. 75 (1998) 1228.

[426] G. W. Slater, C. Desruisseaux, S. J. Hubert, J. F. Mercier, J. Labrie, J. Boileau, F. Tessier, M. P. Pepin, Theory of DNA electrophoresis: a look at some current challenges, Electrophoresis 21 (2000) 3873.

[427] G. A. Griess, E. Rogers, P. Serwer, Application of the concept of an electrophoretic ratchet, Electrophoresis 22 (2001) 981.

[428] M. Stopa, Charging ratchets, Submitted for publication.

[429] M. Di Ventra, G. Papp, C. Coluzza, A. Baldereschi, P. A. Schulz, Indented barrier resonant tunneling rectifiers, J. Appl. Phys. 80 (1996) 4174.

[430] T. A. J. Duke, R. H. Austin, Microfabricated sieve for the continuous sorting of macromolecules, Phys. Rev. Lett. 80 (1998) 1552.

[431] T. Duke, Separation techniques, Curr. Opin. Chem. Biol. 2 (1998) 592.

[432] I. Derényi, R. D. Astumian, ac-separation of particles by biased Brownian motion in a two-dimensional sieve, Phys. Rev. E 58 (1998) 7781. 
[433] W. D. Volkmuth, R. H. Austin, DNA electrophoresis in microlithographic arrays, Nature 358 (1992) 600.

[434] A. van Oudenaarden, S. G. Boxer, Brownian ratchet: molecular separation in lipid bilayers supported on patterned arrays, Science 285 (1999) 1046.

[435] A. Lorke, S. Wimmer, B. Jager, J. P. Kotthaus, W. Wegschneider, M. Bichler, Far-infrared and transport properties of antidot arrays with broken symmetry, Physica B 249 (1998) 312.

[436] E. A. Early, A. F. Clark, C. J. Lobb, Physical basis for half-integral Shapiro steps in a dc SQUID, Physica C 245 (1995) 308.

[437] S. Lifson, J. L. Jackson, On the self-diffusion of ions in polyelectrolytic solution, J. Chem. Phys. 36 (1962) 2410.

[438] A. Ajdari, J. Prost, Free-flow electrophoresis with trapping by a transverse inhomogeneous field, Proc. Natl. Acad. Sci. USA 88 (1991) 4468.

[439] A. Ghosh, Diffusion rate for a Brownian particle in a cosine potential in the presence of colored noise, Phys. Lett. A 187 (1994) 54.

[440] A. N. Malakhov, Acceleration of Brownian particle diffusion parallel to a fast random field with a short spatial period, Tech. Phys. Lett. 24 (1998) 833.

[441] I. Claes, C. Van den Broeck, Stochastic resonance for dispersion in oscillatory flows, Phys. Rev. A 44 (1991) 4970.

[442] I. Claes, C. Van den Broeck, Dispersion of particles in periodic media, J. Stat. Phys. 70 (1993) 1215.

[443] Y. W. Kim, W. Sung, Does stochastic resonance occur in periodic potentials?, Phys. Rev. E 57 (1998) R6237.

[444] H. Gang, A. Daffertshofer, H. Haken, Diffusion of periodically forced Brownian particles moving in space-periodic potentials, Phys. Rev. Lett. 76 (1996) 4874.

[445] M. C. Mahato, A. M. Jayannavar, Synchronized first-passages in a double-well system driven by an asymmetric periodic field, Phys. Lett. A 209 (1995) 21.

[446] M. C. Mahato, A. M. Jayannavar, Asymmetric motion in a double well under the action of zero-mean Gaussian white noise and periodic forcing, Phys. Rev. E 55 (1997) 3716.

[447] A. K. Vidybida, A. A. Serikov, Electrophoresis by alternating fields in a nonNewtonian fluid, Phys. Lett. A 108 (1985) 170.

[448] P. Serwer, G. A. Griess, Adaptation of pulsed-field gel electrophoresis for the improved fractionation of spheres, Analytica Chimica Acta 372 (1998) 299.

[449] P. Serwer, G. A. Griess, Advances in the separation of bacteriophages and related particles, J. Chromatography B 722 (1999) 179.

[450] M. J. Chacron, G. W. Slater, Particle trapping and self-focusing in temporally asymmetric ratchets with strong field gradients, Phys. Rev. E 56 (1997) 3446. 
[451] A. Mogliner, M. Mangel, R. J. Baskin, Motion of molecular motor ratcheted by internal fluctuations and protein friction, Phys. Lett. A 237 (1998) 297.

[452] A. V. Zolotaryuk, P. L. Christiansen, B. Norden, A. V. Savin, Y. Zolotaryuk, Pendulum as a model system for driven rotation in moleculear nanoscale machines, Phys. Rev. E 61 (2000) 3256.

[453] D. G. Luchinsky, M. J. Greenall, P. McClintock, Resonant rectification of fluctuations in a Brownian ratchet, Phys. Lett. A 273 (2000) 316.

[454] T. Hondou, Y. Sawada, Comment on "White-noise-induced transport in periodic structures" by J. Luczka et al., Europhys. Lett. 35 (1996) 313.

[455] G. H. Weiss, M. Gitterman, Motion in a periodic potential driven by rectangular pulses, J. Stat. Phys. 70 (1993) 93.

[456] V. Berdichevsky, M. Gitterman, Josephson junction with noise, Phys. Rev. E 56 (1997) 6340.

[457] J. Li, Z. Huang, Transport of particles caused by correlation between additive and multiplicative noise, Phys. Rev. E 57 (1998) 3917.

[458] J. Li, Z. Huang, Net voltage caused by correlated symmetric noises, Phys. Rev. E 58 (1998) 139.

[459] J. Li, Z. Huang, Flux in the case of Gaussian white noises, Comm. Theor. Phys. 30 (1998) 527.

[460] L. Cao, D. Wu, Fluctuation induced transport in a spatially symmetric periodic potential, Phys. Rev. E 62 (2000) 7478.

[461] Y. A. Jia, J. R. Li, Effects of correlated noises on current, Int. J. Mod. Phys. B $14(2000) 507$.

[462] F. Argoul, A. Arneodo, P. Collet, A. Lesne, Transition to chaos in presence of an external periodic field: cross-over effects in the measure of critical exponents, Europhys. Lett. 3 (1987) 643.

[463] P. Collet, A. Lesne, Renormalization group analysis of some dynamical systems with noise, J. Stat. Phys. 57 (1989) 967.

[464] C. Beck, Brownian motion from deterministic dynamics, Physica A 169 (1990) 324.

[465] T. Hondou, S. Sawada, Dynamical behavior of a dissipative particle in a periodic potential subjected to chaotic noise: Retrieval of chaotic determinism with broken parity, Phys. Rev. Lett. 75 (1995) 3269.

[466] C. Grebogi, E. Ott, S. Pelikan, J. Yorke, Strange attractors that are not chaotic, Physica D 13 (1984) 261.

[467] D. D. Pollock, Thermoelectricity, in: Encyclopedia of Physical Science and Technology, Vol. 16, Academic Press, San Diego, 1992. 
[468] N. W. Ashcroft, N. D. Mermin, Solid State Physics, Saunders College, Philadelphia, 1976.

[469] N. G. van Kampen, Relative stability in nonuniform temperature, IBM J. Res. Develop. 32 (1988) 107.

[470] R. Landauer, Motion out of noisy states, J. Stat. Phys. 53 (1988) 233.

[471] A. M. Jayannavar, M. C. Mahato, Macroscopic equation of motion in inhomogeneous media: a microscopic treatment, Pramana-J. Phys. 45 (1996) 369.

[472] M. C. Mahato, T. P. Pareek, A. M. Jayannavar, Enslaving random fluctuations in nonequilibrium systems, Int. J. Mod. Phys. B 10 (1996) 3857.

[473] J. D. Bao, Y. Abe, Y. Z. Zhuo, Inhomogeneous friction leading to current in periodic systems, Physica A 265 (1999) 111.

[474] R. H. Luchsinger, Transport in nonequilibrium systems with position-dependent mobility, Phys. Rev. E 62 (2000) 272.

[475] K. Sekimoto, Temporal coarse graining for systems of Brownian particles with non-constant temperature, J. Phys. Soc. Jpn. 68 (1999) 1448.

[476] M. Matsuo, S. Sasa, Stochastic energetics of non-uniform temperature systems, Physica A 276 (2000) 188.

[477] N. G. van Kampen, Diffusion in inhomogeneous media, Z. Phys. B 68 (1987) 135 .

[478] Y. M. Blanter, M. Büttiker, Rectification of fluctuations in an underdamped ratchet, Phys. Rev. Lett. 81 (1998) 4040.

[479] H. Risken, Vollmer, Brownian motion in periodic potentials in the low-frictionlimit; linear response to an external force, Z. Phys. B 35 (1979) 177.

[480] K. Sekimoto, Kinetic characterization of heat bath and the energetics of thermal ratchet models, J. Phys. Soc. Jpn. 66 (1997) 1234.

[481] T. Hondou, F. Takaga, Irreversible operation in a stalled state of Feynman's ratchet, J. Phys. Soc. Jpn. 67 (1998) 2974.

[482] H. Sakaguchi, Langevin simulation for the Feynman ratchet model, J. Phys. Soc. Jpn. 67 (1998) 709.

[483] H. Sakaguchi, Fluctuation theorem for a Langevin model of the Feynman ratchet, J. Phys. Soc. Jpn. 69 (2000) 104.

[484] C. Jarzynski, O. Mazonka, Feynman's ratchet and pawl: An exactly solvable case, Phys. Rev. E 59 (1999) 6448.

[485] Y.-D. Bao, Directed current of Brownian ratchet randomly circulating between two thermal sources, Physica A 273 (1999) 286.

[486] Y.-D. Bao, Transport induced by dichotomic temperature fluctuations, Comm. Theor. Phys. 34 (2000) 441. 
[487] P. Hänggi, Nonlinear effects of colored nonstationary noise: exact results, Phys. Lett. A 83 (1981) 196.

[488] D. Ryter, Brownian motion in inhomogeneous media and with interacting particles, Z. Phys. B 41 (1981) 39.

[489] J. M. Sancho, M. San Miguel, D. Dürr, Adiabatic elimination for systems of Brownian particles with nonconstant damping coefficients, J. Stat. Phys. 28 (1982) 291.

[490] J. Happel, H. Brenner, Low Reynolds number hydrodynamics, Martinuis Nijhoff Publishers, Dordrecht, 1986.

[491] L. P. Faucheux, A. J. Libchaber, Confined Brownian motion, Phys. Rev. E 49 (1994) 5158.

[492] B. Lin, J. Yu, S. A. Rice, Diffusion of an isolated colloidal sphere confined between flat plates, Colloids and Surfaces A 174 (2000) 121.

[493] C. M. Falco, Phase-space of a driven, damped pendulum (Josephson weak link), Am. J. Phys. 44 (1976) 733.

[494] R. Krishnan, S. Singh, G. W. Robinson, Space-dependent friction in the theory of activated rate processes: The Hamiltonian approach, J. Chem. Phys. 97 (1992) 5516 .

[495] R. Krishnan, S. Singh, G. W. Robinson, Space-dependent friction in the theory of activated rate processes, Phys. Rev. A 45 (1992) 5408.

[496] J.-D. Bao, Y. Abe, Y. Z. Zhuo, Rocked quantum periodic systems in the presence of coordinate-dependent friction, Phys. Rev. E 58 (1998) 2931.

[497] D. Dan, A. M. Jayannavar, M. C. Mahato, Efficiency and current reversals in spatially inhomogeneous ratchets, Int. J. Mod. Phys. 14 (2000) 1585.

[498] D. Dan, M. C. Mahato, A. M. Jayannavar, Multiple current reversals in forced inhomogeneous ratchets, Phys. Rev. E 63 (2001) 056307.

[499] D. Dan, M. C. Mahato, A. M. Jayannavar, Motion in a rocked ratchet with spatially periodic friction, Physica A 296 (2001) 375.

[500] O. Steuernagel, W. Ebeling, V. Calenbuhr, An elementary model for directed active motion, Chaos, Solitons, and Fractals 4 (1994) 1917.

[501] F. Schweitzer, Active Brownian particles: Artificial agents in physics, in: L. Schimansky-Geier, T. Pöschel (Eds.), Lecture Notes in Physics, Vol. 484, Springer, Berlin, 1997.

[502] B. Tilch, F. Schweitzer, W. Ebeling, Directed motion of Brownian particles with internal energy depot, Physica A 273 (1999) 294.

[503] F. Schweitzer, B. Tilch, W. Ebeling, Uphill motion of active Brownian particles in piecewise linear potentials, Eur. Phys. B 14 (2000) 157. 
[504] L. Schimansky-Geier, S. Seefeld, V. Buchholtz, Making spatial structures by ratchets, Ann. Phys. (Leipzig) 9 (2000) 705.

[505] S. Klump, A. Mielke, C. Wald, Noise-induced transport of two coupled particles, Phys. Rev. E 63 (2001) 031914.

[506] Y. Li, X. Wu, Y. Zhou, Directed motion of two-headed Brownian motors, Mod. Phys. Lett. B 14 (2000) 479.

[507] S. Cilla, L. M. Floria, Internal degrees of freedom in a thermodynamical model for intercellular transport, Physica D 113 (1998) 157.

[508] S. Cilla, L. M. Floria, A two-dimensional model for kinesin and dynein stepping along microtubules, Il Nuovo Cimento D 20 (1998) 1761.

[509] T. E. Dialynas, G. Tsironis, Vectorial stochastic motion driven by dichotomous noise, Phys. Lett. A 218 (1996) 292.

[510] I. Derényi, T. Vicsek, The kinesin walk: a dynamic model with elastically coupled heads, Proc. Natl. Acad. Sci. USA 93 (1996) 6775.

[511] T. C. Elston, C. S. Peskin, The role of flexibility in molecular motor function: coupled diffusion in a tilted periodic potential, SIAM J. Appl. Math. 60 (2000) 842 .

[512] T. C. Elston, D. You, C. S. Peskin, Protein flexibility and the correlation ratchet, SIAM J. Appl. Math. 61 (2000) 776.

[513] Y. Osada, H. Okuzaki, H. Hori, A polymer gel with electrically driven motility, Nature 355 (1992) 242.

[514] O. Sandre, L. Gorre-Talini, A. Ajdari, J. Prost, P. Silberzan, Moving droplets on asymmetrically structured surfaces, Phys. Rev. E 60 (1999) 2964.

[515] Y.-X. Li, Brownian motors possessing internal degree of freedom, Physica A 251 (1998) 382.

[516] F. Müller, A. Birner, J. Schilling, U. Gösele, C. Kettner, P. Hänggi, Membranes for micropumps from macroporous silicon, Phys. Stat. Sol. A 182 (2000) 585.

[517] H. Ambaye, K. W. Kehr, Toy model for molecular motors, Physica A 267 (1999) 111.

[518] I. Sokolov, A perturbation approach to transport in discrete ratchet systems, J. Phys. A 32 (1999) 2541.

[519] K. W. Kehr, Z. Koza, Hopping motion of lattice gases through nonsymmetric potentials under strong bias conditions, Phys. Rev. E 61 (2000) 2319.

[520] T. Duke, S. Leibler, Motor protein mechanics: a stochastic model with minimal mechanochemical coupling, Biophys. J. 71 (1996) 1235.

[521] M. E. Fisher, A. B. Kolomeisky, The force exerted by a molecular motor, Proc. Natl. Acad. Sci. USA 96 (1999) 6597. 
[522] M. E. Fisher, A. B. Kolomeisky, Molecular motors and the forces they exert, Physica A 274 (1999) 241.

[523] A. B. Kolomeisky, M. E. Fisher, Periodic sequential kinetic models with jumping, branching and deaths, Physica A 279 (2000) 1.

[524] A. B. Kolomeisky, M. E. Fisher, Extended kinetic models with waiting-time distributions: exact results, J. Chem. Phys. 113 (2000) 10867.

[525] J. Howard, Mechanics of motor proteins and the cytoskeleton, Sinauer Associates, Sunderland, 2001.

[526] M. E. Fisher, A. B. Kolomeisky, Simple mechanochemistry describes the dynamics of kinesin molecules, Proc. Natl. Acad. Sci. USA 98 (2001) 7748.

[527] J. R. Sanchez, F. Family, C. M. Arizmendi, Algorithmic complexity of thermal ratchet motion, Phys. Lett. A 249 (1998) 281.

[528] C. M. Arizmendi, F. Family, Algorithmic complexity and efficiency of a ratchet, Physica A 269 (1999) 285.

[529] http://seneca.fis.ucm.es/parr/.

[530] C. Van den Broeck, P. Reimann, R. Kawai, P. Hänggi, Coupled Brownian motors, in: D. Reguera, J. M. Rubi, J. M. G. Vilar (Eds.), Lecture Notes in Physics, Vol. 527, 'Statistical Mechanics and Biocomplexity', Springer, Berlin, 1999.

[531] G. P. Harmer, D. Abbott, Losing strategies can win by Parrondo's paradox, Nature 402 (1999) 864.

[532] G. P. Harmer, D. Abbott, Parrondo's paradox, Stat. Sci. 14 (1999) 206.

[533] G. P. Harmer, D. Abbott, P. G. Taylor, J. M. R. Parrondo, Parrondo's paradoxical games and the discrete Brownian ratchet, in: D. Abbott, L. Kish (Eds.), Proc. 2nd Int. Conf. on Unsolved Problems of Noise, AIP Proc. 511, 2000.

[534] G. P. Harmer, D. Abbott, P. G. Taylor, C. E. M. Pearce, J. M. R. Parrondo, Information entropy and Parrondo's discrete-time ratchet, in: D. S. Broomhead, E. A. Luchinskaya, P. V. E. McClintock (Eds.), Proc. Stochaos, AIP Proc. 502, 2000

[535] G. P. Harmer, D. Abbott, P. G. Taylor, The paradox of Parrondo's games, Proc. R. Soc. Lond. A 456 (2000) 1.

[536] J. M. R. Parrondo, G. P. Harmer, D. Abbott, New paradoxical games based on Brownian ratchets, Phys. Rev. Lett. 85 (2000) 5226.

[537] R. Toral, Cooperative Parrondo's games, Fluct. Noise Lett. 1 (2001) L7.

[538] J.-P. Bouchaud, A. Georges, Anomalous diffusion in disordered media: statistical mechanisms, models and physical applications, Phys. Rep. 195 (1990) 127.

[539] S. I. Denisov, W. Horsthemke, Mean first-passage time for an overdamped particle in a disordered force field, Phys. Rev. E 62 (2000) 3311. 
[540] F. Marchesoni, Transport properties in disordered ratchet potentials, Phys. Rev. E 56 (1997) 2492.

[541] R. Alicki, Disordered Markovian Brownian ratchets, Phys. Rev. E 60 (1999) 2559 .

[542] M. N. Popescu, C. M. Arizmendi, A. L. Salas-Brito, F. Family, Disorder induced diffusive transport in ratchets, Phys. Rev. Lett 85 (2000) 3321.

[543] Y. Jia, S. N. Yu, J. R. Li, Effects of random potential on transport, Phys. Rev. E 63 (2001) 052101.

[544] K. Sekimoto, S. Sasa, Complementary relations for irreversible process derived from stochastic energetics, J. Phys. Soc. Jpn. 66 (1997) 3326.

[545] K. Sekimoto, Langevin equation and thermodynamics, Prog. Theor. Phys. Supplement 130 (1998) 17.

[546] T. Hondou, K. Sekimoto, Unattainability of Carnot efficiency in the Brownian heat engine, Phys. Rev. E 62 (2000) 6021.

[547] S. Velasco, J. M. M. Roco, A. Medina, A. C. Hernandez, Feynman's ratchet optimization: maximum power and maximum efficiency regimes, J. Phys. D 34 (2001) 1000.

[548] J.-D. Bao, Variational path-integral approach to current and efficiency with quantum correction, Phys. Lett. A 247 (1998) 380.

[549] H. Kamegawa, T. Hondou, F. Takagi, Energetics of forced thermal ratchets, Phys. Rev. Lett. 80 (1998) 5251.

[550] I. Sokolov, Irreversible and reversible modes of operation of deterministic ratchets, Phys. Rev. E 63 (2001) 021107.

[551] A. Parmeggiani, F. Jülicher, A. Ajdari, J. Prost, Energy transduction of isothermal ratchets: generic aspects and specific examples close to and far from equilibrium, Phys. Rev. E 60 (1999) 2127.

[552] I. M. Sokolov, Ideally efficient irreversible molecular gears, cond-mat/0002251.

[553] I. Derényi, R. D. Astumian, Efficiency of Brownian heat engines, Phys. Rev. E 59 (1999) R6219.

[554] K. Sekimoto, F. Takagi, T. Hondou, Carnot's cycle for small systems: irreversibility and cost of operations, Phys. Rev. E 62 (2000) 7759.

[555] I. M. Sokolov, Reversible fluctuation rectifier, Phys. Rev. E 60 (1999) 4946.

[556] F. Takagi, T. Hondou, Thermal noise can facilitate energy conversion by a ratchet system, Phys. Rev. E 60 (1999) 4954.

[557] F. Jülicher, J. Prost, Cooperative molecular motors, Phys. Rev. Lett. 75 (1995) 2618. 
[558] J. Buceta, J. M. Parrondo, C. Van den Broeck, F. J. de la Rubia, Negative resistance and anomalous hysteresis in a collective molecular motor, Phys. Rev. E $61(2000) 6287$.

[559] R. Lipowsky, T. Harms, Molecular motors and nonuniform ratchets, Eur. Biophys. J. 29 (2000) 542.

[560] M. Bier, R. D. Astumian, Biased Brownian motors as the operating principle for microscopic engines, Bioelectrochem. Bioenerg. 39 (1996) 67.

[561] I. Derényi, M. Bier, R. D. Astumian, Generalized efficiency and its application to microscopic engines, Phys. Rev. Lett. 83 (1999) 903.

[562] M. Bier, Motor proteins: mechanochemical energy transduction on the microscopic scale, Acta Physica Polonica B 32 (2001) 287.

[563] A. C. Hernandez, A. Medina, J. M. M. Roco, J. A. White, S. Velasco, Unified optimization criterion for energy converters, Phys. Rev. E 63 (2001) 037102.

[564] T. Humphrey, R. Newbury, R. Taylor, H. Linke, Reversible quantum heat engines, Submitted for publication.

[565] F. L. Curzon, B. Ahlborn, Efficiency of a Carnot engine at maximum power output, Am. J. Phys. 43 (1975) 22.

[566] B. Andresen, Finite-Time Thermodynamics, University of Copenhagen Press, Copenhagen, 1983.

[567] R. S. Berry, V. A. Kazakov, S. Sieniutycz, Z. Szwast, A. M. Tsirlin, Thermodynamics Optimization of Finite-Time Processes, Wiley, Chichester, 1999.

[568] J. Howard, Molecular motors: structural adaptation to cellular functions, Nature 389 (1997) 561.

[569] A. D. Mehta, M. Rief, J. A. Spudich, D. A. Smith, R. M. Simmons, Singlemolecule biomechanics with optical methods, Science 283 (1999) 1689.

[570] M. Meister, S. R. Caplan, H. C. Berg, Dynamics of a tightly coupled mechanism for flagellar rotation, Biophys. J. 55 (1989) 905.

[571] C. Doering, B. Ermentrout, G. Oster, Rotary DNA motors, Biophys. J. 69 (1995) 2256.

[572] T. C. Elston, G. Oster, Protein turbines. I: The bacterial flagellar motor, Biophys. J. 73 (1997) 703.

[573] T. Elston, H. Wang, G. Oster, Energy transduction in ATP synthase, Nature 391 (1998) 510.

[574] H. C. Berg, Keeping up with the F1-ATPase, Nature 394 (1998) 324.

[575] http://www.borisylab.nwu.edu/pages/supplemental/mtfr.html. 
[576] J. Howard, F. Gittes, Motor proteins, in: H. Flyvbjerg, J. Hertz, M. J. Jensen, O. G. Mouritsen, K. Sneppen (Eds.), Physics of Biological Systems; from Molecules to Species. Lecture Notes in Physics, Vol. 366, Springer, Berlin, 1997, p. 155 .

[577] S. M. Block, Leading the procession: new insights into kinesin motors, J. Cell. Biol. 140 (1998) 1281.

[578] K. Svoboda, C. F. Schmidt, B. J. Schnapp, S. M. Block, Direct observation of kinesin stepping by optical trapping interferometry, Nature 365 (1993) 721.

[579] S. P. Gilbert, M. R. Webb, M. Brune, K. A. Johnson, Pathway of processive ATP hydrolysis by kinesin, Nature 373 (1995) 671.

[580] J. Gelles, B. J. Schnapp, M. P. Sheetz, Tracking kinesin-driven movements with nanometer-scale precision, Nature 331 (1988) 450.

[581] E. P. Sablin, F. J. Kull, R. Cooke, R. D. Vale, R. J. Fletterick, Crystal structure of the motor domain of the kinesin-related motor ncd, Nature 380 (1996) 555.

[582] J. T. Finer, R. S. Simmons, J. A. Spudich, Single myosin molecule mechanics: Piconewton forces and nanometer steps, Nature 368 (1994) 113.

[583] R. A. Cross, Reversing the kinesin ratchet - a diverting tail, Nature 389 (1997) 15.

[584] U. Henningsen, M. Schliwa, Reversal in the direction of movement of a molecular motor, Nature 389 (1997) 93.

[585] J. Howard, A. J. Hudspeth, R. D. Vale, Movement of microtubules by single kinesin molecules, Nature 342 (1989) 154.

[586] E. Mandelkow, A. Hoenger, Structure of kinesin and kinesin-microtubule interactions, Current Opinion in Cell Biology 11 (1999) 34.

[587] K. Svoboda, S. M. Block, Force and velocity measured for single kinesin molecules, Cell 77 (1994) 773.

[588] K. Fukui, The path of chemical reactions - the IRC approach, Acc. Chem. Res. 14 (1981) 363 .

[589] H. Köppel, W. Domcke, L. S. Cederbaum, Multimode molecular dynamics beyond the Born-Oppenheimer approximation, Adv. Chem. Phys. 57 (1984) 59.

[590] P. W. Atkins, Physical Chemistry, 3rd Edition, Oxford University Press, Oxford, 1986.

[591] D. G. Truhlar, Potential energy surfaces, in: Encyclopedia of Physical Science and Technology, Vol. 13, Academic Press, San Diego, 1992.

[592] R. Daudel, Quantum chemistry, in: Encyclopedia of Physical Science and Technology, Vol. 13, Academic Press, San Diego, 1992.

[593] J. Michl, Organic chemical systems, theory, in: Encyclopedia of Physical Science and Technology, Vol. 12, Academic Press, San Diego, 1992. 
[594] C. Schütte, Conformational dynamics: modeling, theory, algorithm, and applications to biomolecules, Habilitation thesis, Konrad-Zuse-Zentrum für Informationstechnik Berlin (Germany), unpublished, 1999.

[595] H. Frauenfelder, P. G. Wolynes, Rate theories and puzzles of hemeprotein kinetics, Science 229 (1985) 337.

[596] H. Frauenfelder, S. G. Sligar, P. G. Wolynes, The energy landscapes and motions of proteins, Science 254 (1991) 1598.

[597] K. Tawada, K. Sekimoto, Protein friction exerted by motor enzymes through a weak-binding interaction, J. Theor. Biol. 150 (1991) 193.

[598] R. Lipowsky, Molecular motors and stochastic models, in: J. A. Freund, T. Pöschel (Eds.), Lecture Notes in Physics, Vol. 557, Springer, Berlin, 2000.

[599] A. Parmeggiani, F. Jülicher, L. Peliti, J. Prost, Detachment of molecular motors under tangential loading, Submitted for publication.

[600] G. Lattanzi, A. Maritan, Force dependence of the Michaelis constant in a twostate ratchet model for molecular motors, Phys. Rev. Lett 86 (2001) 1134.

[601] F. Jülicher, Force and motion generation of molecular motors: a generic description, in: S. C. Müller, J. Parisi, W. Zimmermann (Eds.), Lecture Notes in Physics: Transport and structure: Their competitive roles in Biophysics and Chemistry, Springer, Berlin, 1999.

[602] M. Kikkawa, T. Ishikawa, T. Wakabayashi, N. Hirokawa, Three-dimensional structure of the kinesin head-microtubule complex, Nature 376 (1995) 274.

[603] R. F. Fox, Rectified Brownian movement in molecular and cell biology, Phys. Rev. E 57 (1998) 2177.

[604] J. A. Spudich, How molecular motors work, Nature 372 (1994) 515.

[605] K. Kitamura, M. Tokunaga, A. H. Iwane, T. Yanagida, A single myosin head moves along an actin filament with regular steps of 5.3 nanometers, Nature 397 (1999) 129.

[606] A. D. Mehta, R. S. Rock, M. Rief, J. A. Spudich, M. S. Mooseker, R. E. Cheney, Myosin-V is a processive actin-based motor, Nature 400 (1999) 590.

[607] B. J. Schnapp, Two heads are better than one, Nature 373 (1995) 655.

[608] M. J. Schnitzer, S. M. Block, Kinesin hydrolyses one ATP per 8-nm step, Nature 388 (1997) 386.

[609] K. Svoboda, P. P. Mitra, S. M. Block, Fluctuation analysis of motor protein movement and single enzyme kinetics, Proc. Natl. Acad. Sci. USA 91 (1994) 11782 .

[610] M. J. Schnitzer, S. M. Block, Statistical kinetics of processive enzymes, Cold Spring Harb. Symp. Quant. Biol. 60 (1995) 793. 
[611] A. F. Huxley, R. M. Simmons, Proposed mechanism of force generation in striated muscle, Nature 233 (1971) 533.

[612] D. A. Smith, S. Sicilia, The theory of sliding filament models for muscle contraction. I. The two-state model, J. Theor. Biol 127 (1987) 1.

[613] E. Pate, R. Cooke, A model of crossbridge action: the effecs of ATP, ADP and $\mathrm{P}_{i}$, J. Muscle Res. Cell Motil. 10 (1989) 181.

[614] E. Pate, R. Cooke, Simulation of stochastic processes in motile crossbridge systems, J. Muscle Res. Cell Motil. 12 (1991) 376.

[615] E. Pate, R. Cooke, H. White, Determination of the myosin step size from mechanical and kinetic data, Proc. Natl. Acad. Sci. USA 90 (1993) 2451.

[616] K. Sekimoto, K. Tawada, Extended time correlation of in vitro motility by motor protein, Phys. Rev. Lett. 75 (1995) 180.

[617] N. Thomas, R. A. Thornhill, The physic of biological molecular motors, J. Phys. D $31(1998) 253$.

[618] C. J. Barclay, A weakly coupled version of the Huxley crossbridge model can simulate energetics of amphibian and mammalian skeletal muscle, J. Muscle. Res. Cell M. 20 (1999) 163.

[619] F. Jülicher, J. Prost, Molecular motors: from individual to collective behavior, Prog. Theor. Phys. Supplement 130 (1998) 9.

[620] J. L. Marin, M. Huerta, J. Muniz, X. Trujillo, Comment on "Coopertaive molecular motors", Phys. Rev. Lett. 83 (1999) 5403.

[621] F. Jülicher, J. Prost, Jülicher and Prost reply, Phys. Rev. Lett. 83 (1999) 5404.

[622] F. Jülicher, J. Prost, Spontaneous oscillations in collective molecular motors, Phys. Rev. Lett. 78 (1997) 4510.

[623] D. Riveline, A. Ott, F. Jülicher, D. Winkelmann, O. Cardoso, J. Lacapere, S. Magnusdottir, J. Viovy, L. Gorre-Tallini, J. Prost, Acting on actin: the electric motility assay, Eur. Biophys. J. 27 (1998) 403.

[624] K. Yasuda, Y. Shindo, S. Ishiwata, Synchronous behavior of spontaneous oscillations of sacromeres in skeletal myofibrils under isotonic conditions, Biophys. J. 70 (1996) 1823.

[625] H. Fujita, S. Ishiwata, Spontaneous oscillatory contraction without regulatory proteins in actin filament-reconstituted fibres, Biophys. J. 75 (1998) 1439.

[626] S. Camalet, F. Jülicher, J. Prost, Self-organized beating and swimming of internally driven filaments, Phys. Rev. Lett. 82 (1999) 1590.

[627] A. T. Winfree, The Geometry of Biological Time, Springer, Berlin, 1980.

[628] G. Nicolis, I. Prigogine, Self Organization in Nonequilibrium Systems, Springer, Berlin, 1981. 
[629] Y. Kuramoto, Chemical Oscillations, Waves, and Turbulence, Springer, Berlin, 1984.

[630] C. Vidal, A. Pacault, Nonequilibrium Dynamics in Chemical Systems, Springer, Berlin, 1988.

[631] L. Glass, M. C. Mackey, From Clocks to Chaos, Princeton University Press, Princeton NJ, 1988.

[632] A. Vilfan, E. Frey, F. Schwabl, Elastically coupled molecular motors, Eur. Phys. J. B 3 (1998) 535.

[633] A. Vilfan, E. Frey, F. Schwabl, Force-velocity relation for a two-state crossbridge model for molecular motors, Europhys. Lett. 45 (1999) 283.

[634] http://www.physik.tu-muenchen.de/ avilfan/ecmm/.

[635] I. Derényi, T. Vicsek, Realistic models of biological motion, Physica A 249 (1998) 397.

[636] W. Hua, E. C. Young, M. L. Fleming, J. Gelles, Coupling of kinesin steps to ATP hydrolysis, Nature 388 (1997) 390.

[637] C. M. Coppin, D. W. Pierce, L. Hsu, R. D. Vale, The load dependence of kinesin's mechanical cycle, Proc. Natl. Acad. Sci. USA 94 (1997) 8539.

[638] S. Rice, A. W. Lin, D. Safer, C. L. Hart, N. Naber, B. O. Carragher, S. M. Cain, E. Pechatnikova, E. M. Wilson-Kubalek, M. Wittaker, E. Pate, R. Cooke, E. W. Taylor, R. A. Milligan, R. D. Vale, A structural change in the kinesin motor protein that drives motility, Nature 402 (1999) 778.

[639] J. Gelles, E. Berliner, E. C. Young, H. K. Mahtani, B. Perez-Ramirez, K. Anderson, Structural and functional features of one- and two-headed biotinated kinesin derivatives, Biophys. J. 68 (1995) 276s.

[640] E. Berliner, E. C. Young, K. Anderson, H. K. Mahtani, J. Gelles, Failure of a single-headed kinesin to track parallel to microtubule protofilaments, Nature 373 (1995) 718.

[641] R. D. Vale, T. Funatsu, D. W. Pierce, L. Romberg, Y. Harada, T. Yanagida, Direct observation of single kinesin molecules moving along microtubules, Nature 380 (1996) 451.

[642] Y. Okada, N. Hirokawa, A processive single-headed motor: kinesin superfamily protein KIF1A, Science 283 (1999) 1152.

[643] http://www.sciencemag.org/feature/data/985876.shl].

[644] H.-X. Zhou, Y. Chen, Chemically driven motility of Brownian particles, Phys. Rev. Lett. 77 (1996) 194.

[645] S. M. Block, K. Svoboda, Analysis of high resolution recordings of motor movement, Biophys. J. 68 (1995) 230s. 
[646] K. Visscher, M. J. Schnitzer, S. M. Block, Single kinesin molecules studied with a molecular force clamp, Nature 400 (1999) 184.

[647] G. N. Stratopoulos, T. E. Dialynas, G. Tsironis, Directional Newtonian motion and reversal of molecular motors, Phys. Lett. A 252 (1999) 151.

[648] R. D. Astumian, I. Derényi, A chemically reversible Brownian motor: application to kinesin and ncd, Biophys. J. 77 (1999) 993.

[649] R. D. Astumian, The role of thermal activation in motion and force generation by molecular motors, Phil. Trans. R. Soc. London B 355 (2000) 511.

[650] R. Lipowsky, Universal aspects of the chemomechanical coupling for molecular motors, Phys. Rev. Lett. 85 (2000) 4401.

[651] A. Libchaber, Genome stability, cell motility, and force generation, Prog. Theor. Phys. Supplement 130 (1998) 1.

[652] E. Meyerhöfer, J. Howard, The force generated by a single kinesin molecule against an elastic load, Proc. Natl. Acad. Sci. USA 92 (1995) 574.

[653] A. Houdusse, H. L. Sweeney, Myosin motors: missing structures and hidden springs, Curr. Opin. Struct. Biol. 11 (2001) 182.

[654] F. Oosawa, Sliding and ATPase, J. Biochem. 118 (1995) 863.

[655] F. Oosawa, The loose coupling mechanism in molecular machines of living cells, Genes to Cells 5 (2000) 9.

[656] T. Yanagida, K. Kitamura, H. Tanaka, A. H. Iwane, S. Esaki, Single molecule analysis of the actomyosin motor, Curr. Opin. Cell Biol. 12 (2000) 20.

[657] E. W. Taylor, Variations on the theme of movement, Nature 361 (1993) 115.

[658] R. Dümcke, H. Spohn, The proper form of the generator in the weak coupling limit, Z. Phys. B 34 (1979) 419.

[659] P. Talkner, The failure of the quantum regression hypothesis, Ann. Phys. (New York) 167 (1986) 390.

[660] V. Ambegaokar, Quantum Brownian motion and its classical limit, Ber. Bunsenges. Phys. Chem. 95 (1991) 400.

[661] G. W. Ford, R. F. O'Connell, There is no quantum regression theorem, Phys. Rev. Lett. 77 (1996) 798.

[662] S. Gnutzmann, F. Haake, Positivity violation and initial slips in open systems, Z. Phys. B 101 (1996) 263.

[663] V. Capek, T. Mancal, Isothermal Maxwell daemon as a molecular rectifier, Europhys. Lett. 48 (1999) 365.

[664] H. Grabert, P. Schramm, G.-L. Ingold, Quantum Brownian motion: The functional integral approach, Phys. Rep. 168 (1988) 115. 
[665] V. A. Benderskii, D. E. Makarov, C. A. Wight, Chemical dynamics at low temperatures, Adv. Chem. Phys. 88 (1994) 1.

[666] T. Dittrich, P. Hänggi, G.-L. Ingold, B. Kramer, G. Schön, W. Zwerger, Quantum transport and dissipation, Wiley-VCH Verlag GmBH, Weinheim, 1998, Ch. 4.

[667] M. Grifoni, P. Hänggi, Driven quantum tunneling, Phys. Rep. 304 (1998) 229.

[668] A. Papoulis, Probability, random variables, and stochastic processes, McGraw Hill, New York, 1965.

[669] G. Schön, A. D. Zaikin, Quantum coherent effects, phase transitions and the dissipative dynamics of ultra small tunnel junctions, Phys. Rep. 198 (1990) 237.

[670] R. F. O'Connell, Dissipative and fluctuation phenomena in quantum mechanics with applications, Int. J. Quant. Chem. 58 (1996) 569.

[671] I. R. Senitzky, Dissipation in quantum mechanics. The harmonic oscillator, Phys. Rev. 119 (1960) 670.

[672] H. Lamb, On a peculiarity of the wave-system due to the free vibrations of a nucleus in an extended medium, Proc. London. Math. Soc. 32 (1900) 208.

[673] N. G. van Kampen, Contribution to the quantum theory of light scattering, Dan. Mat. Fys. Medd. 26 (no. 15) (1951) 1.

[674] F. Schwabl, W. Thirring, Quantum theory of laser radiation, Ergeb. exakt. Naturwiss. 36 (1964) 219.

[675] N. N. Bogolyubov, Elementary example for establishing statistical equilibrium in a system coupled to a thermostat, in: On some statistical methods in mathematical physics, Publ. Acad. Sci. Ukr. SSR, 1945, pp. 115-137, [In Russian].

[676] P. Reimann, M. Grifoni, P. Hänggi, Quantum ratchets, Phys. Rev. Lett. 79 (1997) 10.

[677] P. Reimann, M. Grifoni, P. Hänggi, Adiabatically rocked quantum ratchets, in: J. B. Kadtke, A. Bulsara (Eds.), Applied Nonlinear Dynamics and Stochastic Systems near the Millennium, AIP Proc. 411, 1997.

[678] H. Grabert, P. Olschowski, U. Weiss, Quantum rates for dissipative systems at finite temperatures, Phys. Rev. B 36 (1987) 1931.

[679] E. Freidkin, P. S. Riseborough, P. Hänggi, Quantum tunneling at low temperatures: results for weak damping, Z. Phys. B 64 (1986) 237, erratum: Z. Phys. B 67 (1987) 271.

[680] P. Hänggi, W. Hontscha, Unified approach to the quantum-Kramers reaction rate, J. Chem. Phys 88 (1988) 4094.

[681] S. Jorda, Quanten auf der Kippratsche, Phys. Bl. 53 (1997) 975.

[682] H. Linke, Von Dämonen und Elektronen, Phys. Bl. 56 (2000) 45.

[683] A farewell to wire?, Wire Industry, March issue (2000) 137-140. 
[684] M. Holthaus, D. W. Hone, Localization effects in ac-driven tight-binding lattices, Philos. Mag. B 74 (1996) 105.

[685] I. Goychuk, M. Grifoni, P. Hänggi, Nonadiabatic quantum Brownian rectifiers, Phys. Rev. Lett. 81 (1998) 649, erratum: Phys. Rev. Lett. 81 (1998) 2837.

[686] I. Goychuk, P. Hänggi, Quantum rectifiers from harmonic mixing, Europhys. Lett. 43 (1998) 503.

[687] I. Goychuck, private communication.

[688] S. Yukawa, M. Kikuchi, G. Tatara, H. Matsukawa, Quantum ratchets, J. Phys. Soc. Jpn. 66 (1997) 2953.

[689] R. Roncaglia, G. Tsironis, Discrete quantum motors, Phys. Rev. Lett. 81 (1998) 10.

[690] S. Yukawa, G. Tatara, M. Kikuchi, H. Matsukawa, Quantum ratchet, Physica B 284-288 (2000) 1896.

[691] G. Tatara, M. Kikuchi, S. Yukawa, H. Matsukawa, Dissipation enhanced asymmetric transport in quantum ratchets, J. Phys. Soc. Jpn. 67 (1998) 1090.

[692] H. Linke, T. E. Humphrey, R. P. Taylor, A. P. Micolich, R. Newbury, Chaos in quantum ratchets, Physica Scripta T90 (2001) 54.

[693] V. Ambegaokar, U. Eckern, G. Schön, Quantum dynamics of tunneling between superconductors, Phys. Rev. Lett. 48 (1982) 1745.

[694] A. I. Larkin, Y. N. Ovchinikov, Decay of supercurrent in tunnel junctions, Phys. Rev. B 28 (1983) 6281.

[695] U. Eckern, G. Schön, V. Ambegaokar, Quantum dynamics of a superconducting tunnel junction, Phys. Rev. B 30 (1984) 6419.

[696] V. S. Letokhov, V. G. Minogin, B. D. Pavlik, Cooling and capture of atoms and molecules by a resonant light field, Sov. Phys. JETP 45 (1977) 698.

[697] A. Hemmerich, T. W. Hänsch, Two-dimensional atomic crystals bound by light, Phys. Rev. Lett. 70 (1993) 410.

[698] G. Grynberg, B. Lounis, P. Verkerk, J.-Y. Courtois, C. Salomon, Quantized motion of cold Cesium atoms in two- and three-dimensional optical potentials, Phys. Rev. Lett. 70 (1993) 2249.

[699] M. G. Prentiss, Bound by light, Science 260 (1993) 1078.

[700] S. R. Wilkinson, C. F. Bharucha, K. W. Madison, Q. Niu, M. G. Raizen, Observation of atomic Wannier-Stark ladders in an accelerating optical potential, Phys. Rev. Lett. 76 (1996) 4512.

[701] C. L. Allyn, A. C. Gossard, W. Wiegmann, A new rectifying semiconductor structure by molecular epitaxy, Appl. Phys. Lett. 36 (1980) 373. 
[702] F. Capasso, S. Luryi, W. T. Tsang, C. G. Bethea, B. F. Levine, New transient electrical polarization phenomenon in sawtooth superlattices, Phys. Rev. Lett. 51 (1980) 2318.

[703] A. A. Ignatov, E. Schomburg, K. F. Renk, W. Schatz, J. F. Palmier, F. Mollot, Response of a Bloch oscillator to a THz-field, Ann. Phys. (Leipzig) 3 (1994) 137.

[704] B. J. Keay, S. J. Allen Jr., J. Galan, J. P. Kaminski, K. L. Campman, A. C. Gossard, U. Bhattacharya, M. J. W. Rodwell, Photon-assisted electric field domains and multiphoton-assisted tunneling in semiconductor superlattices, Phys. Rev. Lett. 75 (1995) 4098.

[705] B. J. Keay, S. Zeuner, S. J. Allen Jr., K. D. Maranowski, A. C. Grossard, U. Bhattacharya, M. J. W. Rodwell, Dynamic localization, absolute negative conductance and stimulated multiphoton emission in sequential resonant tunneling semiconductor superlattices, Phys. Rev. Lett. 75 (1995) 4102.

[706] J. B. Majer, M. Grifoni, M. Tusveld, J. E. Mooij, Quantum ratchet effect for vortices, Submitted for publication.

[707] H. Linke, W. Sheng, A. Löfgren, H. Xu, P. Omling, P. E. Lindelof, A quantum dot ratchet: experiment and theory, Europhys. Lett. 44 (1998) 341, erratum: Europhys. Lett. 45 (1999) 406.

[708] H. Linke, Experimental quantum ratchets based on solid state nanostructures, Aust. J. Phys. 52 (1999) 895.

[709] H. Linke, H. Xu, A. Löfgren, W. Sheng, A. Svensson, P. Omling, P. E. Lindelof, R. Newbury, R. P. Taylor, Voltage and temperature limits for the operation of a quantum dot ratchet, Physica B 272 (1999) 61.

[710] H. Linke, W. Sheng, A. Löfgren, A. Svensson, H. Xu, P. Omling, P. E. Lindelof, Electron quantum dot ratchets, Microelectr. Eng. 47 (1999) 265.

[711] M. Rauner, Einbahnstrasse Quantenpunkt, Phys. Bl. 55 (1999) 16.

[712] H. Linke, T. E. Humphrey, A. Löfgren, A. O. Sushkov, R. Newbury, R. P. Taylor, P. Omling, Experimental tunneling ratchets, Science 286 (1999) 2314.

[713] T. Humphrey, A numerical simulation of a quantum ratchet, Master's thesis, University of New South Wales (Sydney, Australia), unpublished, 1999.

[714] H. Linke, P. Omling, From linear to non-linear transport in asymmetric mesoscopic devices, Acta Physica Polonica B 32 (2001) 267.

[715] T. Humphrey, H. Linke, R. Newbury, Pumping heat with quantum ratchets, Submitted for publication.

[716] M. Porto, M. Urbakh, J. Klafter, Atomic scale engines: cars and wheels, Phys. Rev. Lett. 84 (2000) 6058.

[717] M. Porto, Atomic scale engines: taking a turn, Acta Physica Polonica B 32 (2001) 295. 
[718] Z. Zheng, G. Hu, B. Hu, Collective directional transport in coupled nonlinear oscillators without external bias, Phys. Rev. Lett. 86 (2001) 2273.

[719] I. Derényi, T. Vicsek, Cooperative transport of Brownian particles, Phys. Rev. Lett. 75 (1995) 374.

[720] F. Marchesoni, Thermal ratchets in 1+1 dimensions, Phys. Rev. Lett. 77 (1996) 2364 .

[721] Z. Csahok, F. Family, T. Vicsek, Transport of elastically coupled particles in an asymmetric periodic potential, Phys. Rev. E 55 (1997) 5179.

[722] A. V. Savin, G. Tsironis, A. Zolotaryuk, Ratchet and switching effects in stochastic kink dynamics, Phys. Lett. A 229 (1997) 279.

[723] A. V. Savin, G. Tsironis, A. Zolotaryuk, Reversal effects in stochastic kink dynamics, Phys. Rev. E 56 (1997) 2457.

[724] A. V. Zolotaryuk, P. L. Christiansen, B. Norden, A. V. Savin, Soliton and ratchet motions in helices, Cond. Mat. Phys. 2 (1999) 293.

[725] I. Derényi, P. Tegzes, T. Vicsek, Collective transport in locally asymmetric periodic structures, Chaos 8 (1998) 657.

[726] Z. Farkas, P. Tegzes, A. Vukics, T. Vicsek, Transitions in the horizontal transport of vertically vibrated granular layers, Phys. Rev. E 60 (1999) 7022.

[727] http://www.ph.biu.ac.il/ rapaport/java-apps/vibseg.html.

[728] Y. Kuramoto, Self-entrainment of a population of coupled non-linear oscillators, in: Lecture Notes in Physics, Vol. 39, Springer, Berlin, 1975, p. 420.

[729] S. H. Strogatz, Norbert Wiener's brain waves, in: S. Levine (Ed.), Frontiers in Mathematical Biology, Springer, Berlin, 1994.

[730] R. Häussler, R. Bartussek, P. Hänggi, Coupled Brownian rectifiers, in: J. B. Kadtke, A. Bulsara (Eds.), Applied Nonlinear Dynamics and Stochastic Systems near the Millennium, AIP Proc. 411, 1997.

[731] P. Martinoli, P. Lerch, C. Leemann, H. Beck, Arrays of Josephson junctions: Model systems for two-dimensional physics, Jpn. J. Appl. Phys. supplement 263 (1987) 1999.

[732] Y. Aghababaie, G. Menon, M. Plischke, Universal properties of Brownian motors, Phys. Rev. E 59 (1999) 2578.

[733] P. Reimann, R. Kawai, C. Van den Broeck, P. Hänggi, Coupled Brownian motors: Anomalous hysteresis and zero-bias negative conductance, Europhys. Lett. 45 (1999) 545.

[734] P. Reimann, C. Van den Broeck, R. Kawai, Nonequilibrium noise in coupled phase oscillators, Phys. Rev. E 60 (1999) 6402.

[735] C. Van den Broeck, I. Bena, P. Reimann, J. Lehmann, Ann. Phys. (Leipzig) 9 (2000) 713. 
[736] S. E. Mangioni, R. R. Deza, H. S. Wio, Transition from anomalous to normal hysteresis in a system of coupled Brownian motors: a mean-field approach, Phys. Rev. E 63 (2001) 041115.

[737] B. Cleuren, C. Van den Broeck, Ising model for a Brownian donkey, Europhys. Lett. 54 (2001) 1.

[738] K. Alekseev, E. Cannon, J. McKinney, F. Kusmartsev, D. Campbell, Spontaneous dc current generation in a resistively shunted semiconductor superlattice driven by a terahertz field, Phys. Rev. Lett. 80 (1998) 2669.

[739] E. H. Cannon, F. V. Kusmartsev, K. N. Alekseev, D. K. Cambell, Absolute negative conductivity and spontaneous current generation in semiconductor superlattices with hot electrons, Phys. Rev. Lett. 85 (2000) 1302.

[740] R. C. Desai, R. Zwanzig, Statistical mechanics of a nonlinear stochastic model, J. Stat. Phys. 19 (1978) 1.

[741] D. A. Dawson, Critical dynamics and fluctuations for a mean-field model of cooperative behavior, J. Stat. Phys. 31 (1983) 29.

[742] L. L. Bonilla, Stable nonequilibrium probability densities and phase transitions for mean-field models in the thermodynamic limit, J. Stat. Phys. 46 (1987) 659.

[743] S. Strogatz, R. Mirollo, Stability of incoherence in a population of coupled oscillators, J. Stat. Phys. 63 (1991) 613.

[744] J. Garcia-Ojalvo, J. M. Sancho, L. Ramirez-Piscina, A nonequilibrium phase transition with colored noise, Phys. Lett. A 168 (1992) 35.

[745] C. Van den Broeck, J. M. R. Parrondo, R. Toral, Noise induced nonequilibrium phase transitions, Phys. Rev. Lett. 73 (1994) 3395.

[746] A. Becker, L. Kramer, Linear stability analysis for bifurcations in spatially extended systems with fluctuating control parameter, Phys. Rev. Lett. 73 (1994) 955.

[747] S. Ramaswami, Comment on "Noise-induced nonequilibrium phase transitions", Phys. Rev. Lett. 75 (1995) 4786.

[748] G. Grinstein, M. A. Munoz, Y. Tu, Phase structure of systems with multiplicative noise, Phys. Rev. Lett. 76 (1996) 4376.

[749] S. Kim, S. H. Park, C. S. Ryu, Noise-enhanced multistability in coupled oscillator systems, Phys. Rev. Lett. 78 (1997) 1616.

[750] R. Müller, K. Lippert, A. Kühnel, U. Behn, First-order nonequilibrium phase transition in a spatially extended system, Phys. Rev. E 56 (1997) 2658.

[751] S. Mangioni, R. Deza, H. S. Wio, R. Toral, Disordering effects of colored noise in nonequilibrium phase transitions induced by multiplicative noise, Phys. Rev. Lett. 79 (1997) 2389. 
[752] A. A. Zaikin, L. Schimansky-Geier, Spatial patters induced by additive noise, Phys. Rev. E 58 (1998) 4355.

[753] http://www.kawai.phy.uab.edu/research/motor.

[754] T. J. Banys, I. V. Parshelyunas, Y. K. Pozhela, Absolute negative resistance of Gallium-Arsenide in a strong microwave field, Sov. Phys. Semicond. 5 (1972) 1727, [Fiz. Tekh. Poluprovodn. 5 (1971) 1990].

[755] V. V. Pavlovich, E. M. Epstein, Conductivity of a superlattice semiconductor in strong electric fields, Sov. Phys. Semicond. 10 (1976) 1196.

[756] J. Pozhela, Plasma and Current Instabilities in Semiconductors, Pergamon Press, Oxford, 1981.

[757] T. C. Sollner, E. R. Brown, W. D. Goodhue, H. Q. Le, Microwave and millimeterwave resonant-tunneling devices, in: F. Carpasso (Ed.), Springer Series in Electronics and Photonics, Vol. 28: Physics of Quantum electron devices, Springer, Berlin, 1990.

[758] A. A. Ignatov, E. Schomburg, J. Grenzer, K. F. Renk, E. P. Dodin, THz-field induced nonlinear transport and dc voltage generation in a semiconductor superlattice due to Bloch oscillations, Z. Phys. B 98 (1995) 187.

[759] Y. Dakhnovskii, H. Metiu, Absolute negative resistance in double-barrier heterostructures in a strong laser field, Phys. Rev. B 51 (1995) 4193.

[760] R. Aguado, G. Platero, Dynamical localization and absolute negative conductance in an ac-driven double quantum well, Phys. Rev. B 55 (1997) 12860.

[761] L. Hartmann, M. Grifoni, P. Hänggi, Dissipative transport in dc-ac-driven tightbinding lattices, Europhys. Lett. 38 (1997) 497.

[762] I. Goychuk, E. Petrov, V. May, Noise-induced current reversal in a stochastically driven dissipative tight-binding model, Phys. Lett. A 238 (1998) 59.

[763] H. Krömer, Proposed negative-mass microwave amplifier, Phys. Rev. 109 (1959) 1856 .

[764] D. C. Mattis, M. J. Stevenson, Theory of negative-mass cyclotron resonance, Phys. Rev. Lett. 3 (1959) 18.

[765] P. F. Liao, A. M. Glass, L. M. Humphrey, Optically generated pseudo-Stark effect in ruby, Phys. Rev. B 22 (1980) 2276.

[766] A. G. Aronov, B. Z. Spivak, Photoeffect in a Josephson junction, JETP Lett. 22 (1975) 101.

[767] M. E. Gershenzon, M. I. Falei, Absolute negative resistance of a tunnel contact between superconductors with a nonequilibrium quasiparticle distribution function, JETP Lett. 44 (1986) 682.

[768] M. E. Gershenzon, M. I. Falei, Absolute negative resistance in tunnel junctions of nonequilibrium superconductors, Sov. Phys. JETP 67 (1988) 389. 
[769] N. A. Dyatko, I. V. Kochetov, A. P. Napartovich, Absolute negative conductivity of a low-temperature plasma, Sov. Tech. Phys. Lett. 13 (1987) 610.

[770] Z. Rozenberg, M. Lando, M. Rokni, On the possibility of steady state negative mobility in externally ionized gas mixtures, J. Phys. D 21 (1988) 1593.

[771] P. M. Golovinskii, A. I. Shchedrin, Weak-field absolute negative conductivity in the mixture $\mathrm{Xe}: \mathrm{F}_{2}$ ionized by a beam of fast electrons, Sov. Phys. Tech. Phys. $34(1989) 159$.

[772] C. Van den Broeck, R. Kawai, Absorption-desorption phase transition induced by parametric modulation, Phys. Rev. E 57 (1998) 3866.

[773] I. Bena, C. Van den Broeck, Coupled parametric oscillators, Europhys. Lett. 48 (1999) 498.

[774] T. Alarcon, A. Perez-Madrid, J. M. Rubi, Energy transduction in periodically driven non-Hermitian systems, Phys. Rev. Lett. 85 (2000) 3995.

[775] C. R. Doering, A stochastic partial differential equation with multiplicative noise, Phys. Lett. A 122 (1987) 133.

[776] M. Ibanes, J. Garcia-Ojalvo, R. Toral, J. M. Sancho, Noise-induced scenario for inverted phase diagrams, Phys. Rev. Lett. 87 (2001) 020601.

[777] R. Eichhorn, P. Reimann, P. Hänggi, Brownian motion with absolute negative mobility, Submitted for publication.

[778] C. Van den Broeck, unpublished.

[779] S. Shinomoto, Y. Kuramoto, Phase transitions in active rotator systems, Prog. Theor. Phys. 75 (1986) 1105.

[780] S. H. Strogatz, C. M. Marcus, R. M. Westervelt, R. E. Mirollo, Collective dynamics of coupled oscillators with random pinning, Physica D 36 (1989) 23.

[781] H. Sompolinsky, D. Golomb, D. Kleinfeld, Cooperative dynamics in visual processing, Phys. Rev. A 43 (1991) 6990.

[782] J. W. Swift, S. Strogatz, K. Wiesenfeld, Averaging of globally coupled oscillators, Physica D 55 (1992) 239.

[783] D. H. G. Mato, C. Meunier, Clustering and slow switching in globally coupled phase oscillators, Phys. Rev E 48 (1993) 3470.

[784] A. Arenas, C. J. Pérez-Vicente, Exact long-time behavior of a network of phase oscillators under random fields, Phys. Rev. E 50 (1994) 949. 